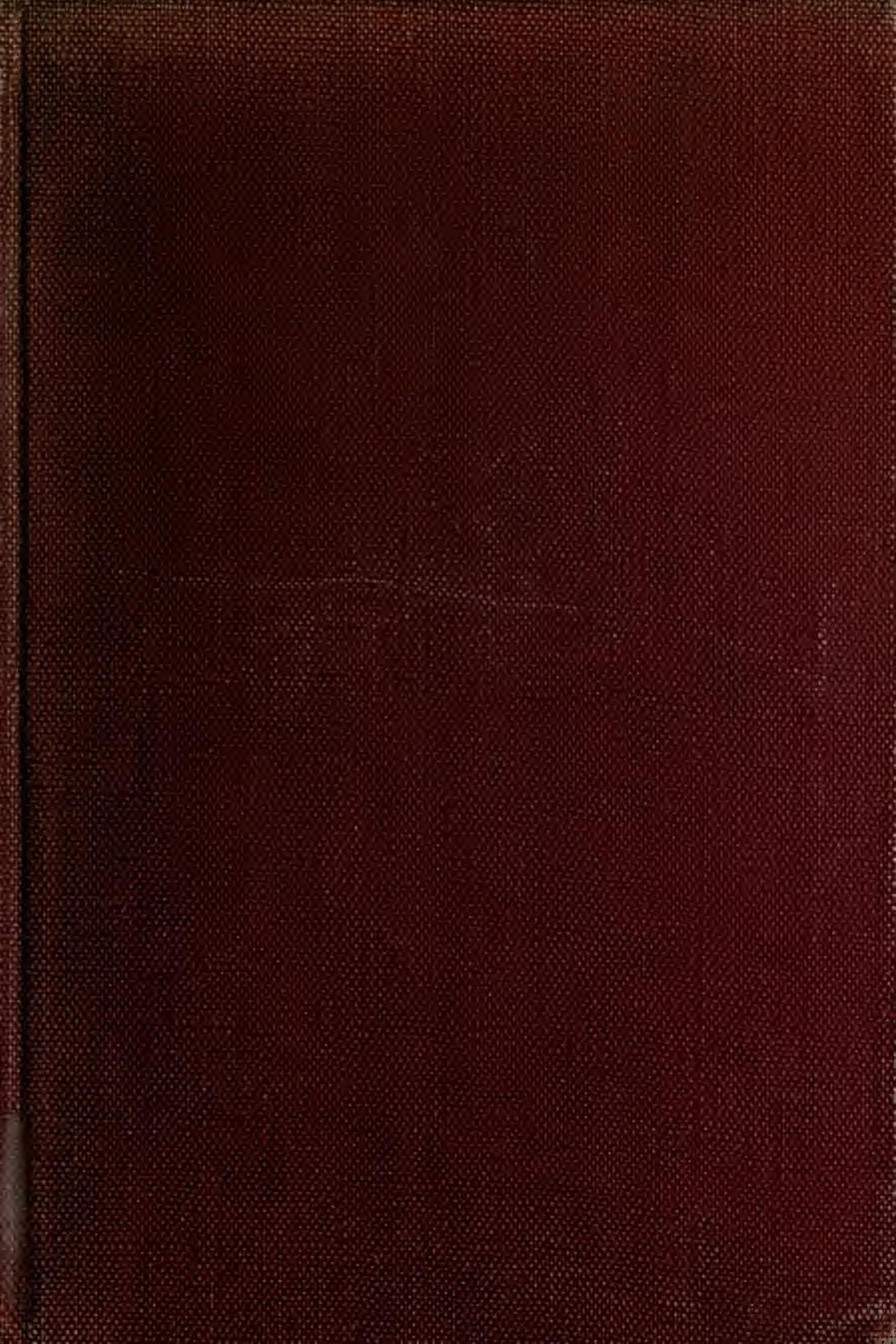



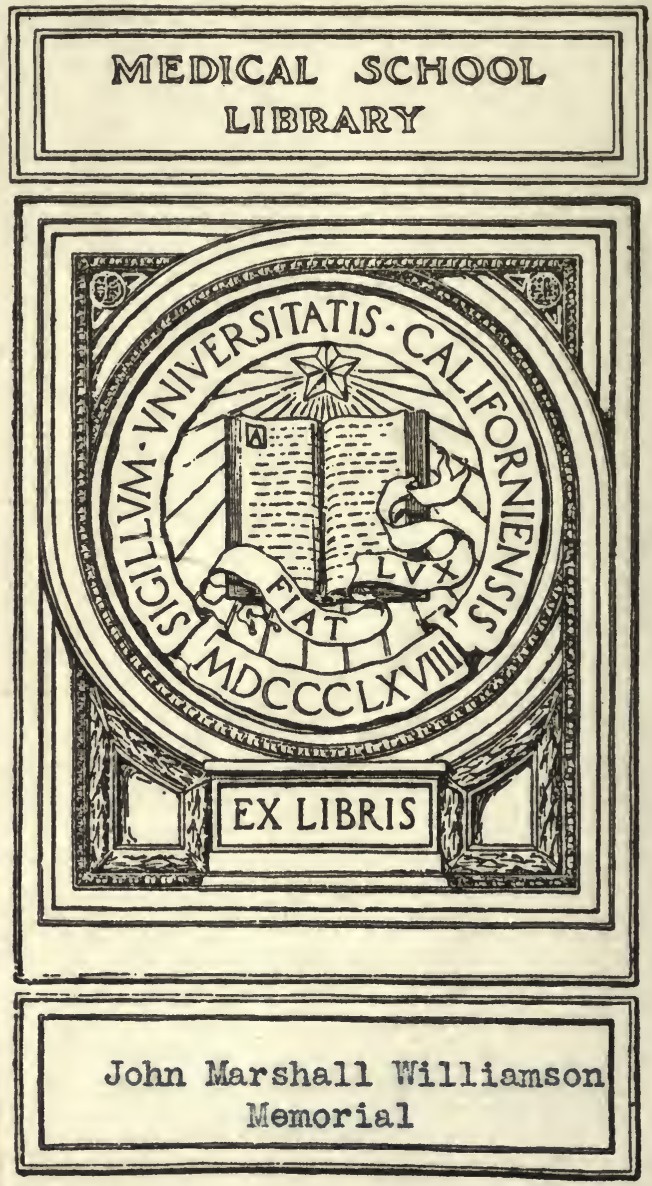

Digitized by the Internet Archive in-2007 with funding from Microsoft Corporation 


$$
\text { - }
$$







\title{
ANATOMY
}

\section{AND PHYSIOLOGY}

\section{FOR NURSES}

\author{
BY \\ LEROY LEWIS, M.D.
}

LATB SURGEON TO AND LECTURER ON ANATUMY AND PHYSIOLOGY FOR NURSES AT THE LEWIS HOSPITAL, BAY CITY, MICHIGAN

Third Edition, Thoroughly Revised

PHILADELPHIA AND LONDON

W. B. SAUNDERS COMPANY

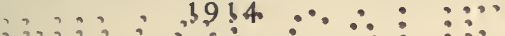

$\because \vdots \quad \vdots . \because \vdots \vdots \ldots \vdots \vdots$

$\because \because$ 
Copyright, x905, by W. B. Saunders and Company. Reprinted Janu. ary, 1906, April, 1907, August, 1908, and February, 1909. Revised, reprinted, and recopyrighted January, 1910.

Reprinted September, 1910, and January, 19x2.

Revised, entirely reset, reprinted, and recopyright $3 d$ September, 19x3.

Copyright, 1913, by W. B. Saunders Company.

Reprinted September, $\mathbf{x}_{9 \mathbf{1}} \mathbf{4}$

PRINTED IN AMERICA

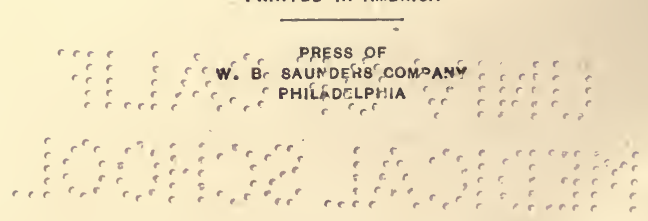




\section{PREFACE TO THIRD EDITION.}

THE first edition of this work was written by the late Dr. Le Roy Lewis upon the request of many of those whom he had instructed in anatomy and physiology. He endeavored to make the text as simple and comprehensive as possible, so that students might be able to grasp the primary principles, after which he felt that they would be sufficiently equipped to apply themselves to future studies.

The works of Gray, Wilson, Deaver, Brubaker, Kimber, and others were freely consulted, and in some instances their language was employed or paraphrased. The publishers furnished many illustrations, which elucidate the text and aid the student in grasping the details of the subject.

Owing to the gratifying reception accorded the previous editions of this work, the present editor has not deemed it wise to depart materially from the general plan and manner of presenting the subject. Some portions have been rewritten, considerable new matter has been incorporated, and the entire book has been thoroughly revised. It is hoped that this new edition will be found to be particularly adapted to the needs of students and practitioners of nursing. 



\section{PREFACE.}

ThE frequent request, on the part of those whom it has been my pleasure to instruct in Anatomy and Physiology during the last few years, to write a book based somewhat upon the plan employed in teaching this subject has been the principal incentive to my action.

The field of anatomy and physiology is already too well cultivated for one, in this brief space, to hope to do more than aid the student in acquiring established facts.

In preparing this work I have endeavored to make the text as simple and comprehensive as could be done, that the students may be able to grasp the primary principles, after which they will have sufficient knowledge to apply themselves to future studies.

The works of Gray, Wilson, Deaver, Brubaker, Kimber, and others have been freely consulted, and in some instances their language has been employed or paraphrased.

Through the kindness of the publishers I have been enabled to make use of many illustrations that 
will be to the student object-lessons and in many instances make the text more comprehensive.

The author desires to acknowledge the aid derived from the sources mentioned, and trusts that the reader will find something to commend in the pages that follow.

LE ROY LEWIS. 


\section{CONTENTS.}

PAGB

INTRODUCTION I I

CHAPTER I.

- Osteology . . . . . . . . . . . . . . 13

Classification of Bones ....... 20

The Spinal Column. . . . . . . . . 22

The Skull . . . . . . . . . . . 24

Thorax and Pelvis ........ . . 30

Bones of the Upper Extremities . . . . . . 34

The Hand . . . . . . . . . . . 37

Bones of the Lower Extremities . . . . . $3^{8}$

The Foot.......... . . 4 I

The Articulations . . . . . . . . 42

Tendons . . . . . . . . . . 46

Adipose Tissue . . . . . . . . . . 47

CHAPTER II.

- The Muscular System .. . . . . . . . 52

Muscles of the Head and Face . . . . . . 55

Muscles of the Neck . . . . . . . . 60

Muscles of the Trunk . . . . . . . . 64

Muscles of the Upper Extremities . . . . . . . 69

Muscles of the Shoulder and Arm . . . . . 69

Muscles of the Forearm . . . . . 72

Muscles of the Hand . . . . . . . . 74

Muscles of the Lower Extremities . . . . $7^{6}$

Muscles of the Leg . . . . . . 82

Muscles of the Foot . . . . . 84

Muscles of the Perineum ....... . 84 
CHAPTER III.

- The Circulatory or Vascular System . . . . 87

The Heart . . . . . . . . . . . . 87

The Arterial System . . . . . . . . . . 94

The Aorta . . . . . . . . . . . . . 96

The Thoracic Aorta . . . . . . . I Io

The Abdominal Aorta . . . . . . . . I II I

The Venous System . . . . . . . . . I I 8

Veins of the Head and Neck . . . . . . I I I

Veins of the Upper Extremities . . . . . I I24

Veins of the Lower Extremities . . . . . I26

Veins of the Trunk ........ . I 27

The Portal Circulation ......... I I30

The Pulmonary Circulation . . . . . . I 30

The Blood . . . . . . . . . . . I I I I

The Vascular System of the Fetus..... I 38

CHAPTER IV.

. The Respiratory System .......... . I44

The Lungs . . . . . . . . . . . I 45

The Diaphragm......... ${ }^{\mathrm{r}} 53$

CHAPTER V.

- The Digestive System ........... I54

The Esophagus and Stomach . . . . . . I62

The Intestines . . . . . . . . . . I65

The Lacteals . . . . . . . . . . . . . I73

The Thoracic Duct ......... . . I75

- Regions of the Abdomen and their Contents . . i i7

CHAPTER VI.

'The Excretory System . . . . . . . . I 80

The Kidneys . . . . . . . . . . . 182

The Ureters . . . . . . . . . . I 86

The Bladder........... . . I86 
The Excretory System (Continued). Pag The Suprarenal Capsule . . . . . . . 190 The Skin . . . . . . . . . . . . 190 The Appendages of the Skin . . . . . I94

CHAPTER VII.

The Nervous System. . . . . . . . . . 198 The Nerves. . . . . . . . . . . 203 The Brain .. . . . . . . . . . 209 The Spinal Cord . . . . . . . . . . 216 The Nerves of the General System . . . . . 2 I9 The Great Sympathetic Nerve... . . . 229

\section{CHAPTER VIII.}

- The Glandular System ... . . . . . . 233

The Lymphatic Glands . . . . . . . . 233

The Liver . . . . . . . . . . . ${ }^{2} 35$

The Gall-bladder . . . . . . . . . 237

The Spleen . . . . . . . . . . ${ }^{2} 37$

The Pancreas. . . . . . . . . . 24I

The Parotid Glands . . . . . . . . 24I

The Submaxillary Glands. . . . . . . . . 243

The Sublingual Glands. . . . . . . . 243

The Thyroid Gland . . . . . . . . 244

The Lacrimal Glands . . . . . . . . 244

The Mesenteric Glands . . . . . . . . 245

The Mammary Glands . . . . . . . 246

The Sebaceous Glands . . . . . . . . 247

The Sudoriparous Glands . . . . . . . 249

CHAPTER IX.

The Membranes of the Body....... . . $25 \mathrm{I}$

CHAPTER $\mathrm{X}$.

The Organs of Special Sense . . . . . . 253

The Organs of Sight . . . . . . . 253 
The Organs of Special Sense (Continued)

The Appendages of the Eye . . . . . $25^{8}$

Physiology of Vision . . . . . . 260 The Organs of Hearing ........26r The Organs of Taste ....... . 264 The Organs of Smell . . . . . . . 266 The Organs of Touch ........ 268

\section{CHAPTER XI.}

The Female Organs of Generation..... $27 \mathrm{I}$ The Vagina . . . . . . . . 274 The Uterus. . . . . . . . . . . 275 Appendages of the Uterus ..... 279 CHAPTER XII.

The Male Organs of Generation ... . . . 287

The Prostate Gland . . . . . . . . 287

Cowper's Glands . . . . . . . . 287 The Penis............ 287 The Testes ............ . 288

The Seminal Vesicles . . . . . . . 290 Semen .. . . . . . . . . . 29I Impregṇation ... . . . . . . . . . 29I

CHAPTER XIII.

Repair and Waste-Nutrition-Animal Heat-PerspiRation-The Power that Supports and Preserves Health . . . . . . . . . . 294 Nutrition . . . . . . . . 296 Animal Heat . . . . . . . . . 297 Perspiration . . . . . . . . . . . 299 The Power that Supports and Preserves Health . 302 


\section{ANATOMY AND PhySIOLOGY}

\section{FOR \\ NURSES}

\section{INTRODUCTION}

In taking up the study of the anatomy of the human body we shall confine ourselves to a general consideration of those branches that most concern the nurse, taking up the anatomy of the various organs and their physiologic functions, and omitting the minute anatomy that is required only of those who desire to perfect themselves in advanced work. The essential points that a nurse should be well acquainted with are: The regions of the body; the internal organs, their names and their location, the relation each organ bears to life. Thus only will we be enabled to understand the conditions of the body in disease.

In applying ourselves to that important subject that concerns the nursing of the sick, we should understand, first what goes to make up the complex organization of the body and, second, by what means we are to obtain this knowledge.

We shall begin our first study by taking up the several systems that constitute the body, so that the nurse may 
become familiar with the technical terms that will be employed in the general work:

The word anatomy is derived from two Greek words, àú, apart, and $\tau \varepsilon \dot{\varepsilon} \mu \nu \varepsilon \iota$, to cut, meaning literally dissection.

The science of anatomy comprises the study of the body as a whole and the relations of its various organs. It is divided into the following branches: osteology, the anatomy of the bones; syndesmology, that of the joints; myology, that of the muscles; angiology, that of the vessels; neurology, that of the nerves; splanchnology, that of the internal viscera; adenology, that of the glands; dermatology, that of the skin; genesiology, that of the generative organs. 


\section{H A P T E R I}

\section{OSTEOLOGY}

WE shall first consider the framework (or osseous system) that supports the body. This is known as "the skeleton" (from the Greek $\sigma \times \varepsilon \lambda \varepsilon \tau o$, a dried body), and gives attachment to the muscles and forms a protection to the internal organs.

Bone.-Bone is the hardest structure in the body; it possesses also a certain degree of toughness and elas-

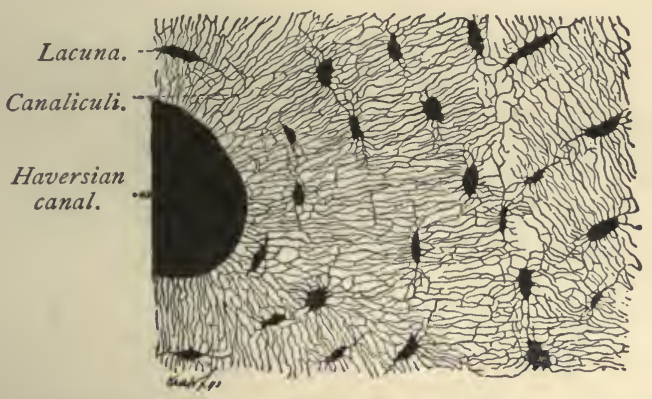

FIG. I.-Portion of a transversely ground disk from the shaft of a human femur; $\times 400$ (Böhm and Davidoff).

ticity. Its color, in a fresh state, is of a pinkish white. On examination it will be found to be composed of two kinds of tissues, one dense and compact, like ivory, the other made up of slender fibers (lamelle) arranged in the form of lattice work, and called cancellated tissue. The former is always external, and the latter internal. These combinations vary in different parts of the body. In 
portions of bone subject to great force the tissues are more compact, while the parts that are not exposed to

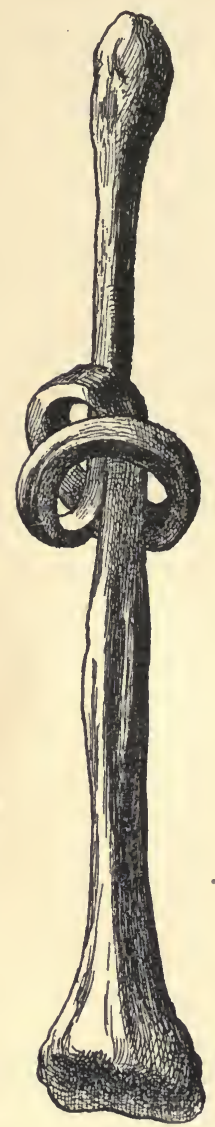

FIG. 2.-Bone tied in knot (Raymond). severe strain are provided with cancellous tissue. Bone tissue contains longitudinal canals that communicate with one another, and are called Haversian canals. They convey bloodvessels for the nutrition of the bone.

All bones are covered with a dense fibrous vascular membrane, the periosteum. The medullary and cancellous cavities of bone are lined with a delicate membrane, the endosteum containing bone-forming cells. In their normal state bones have but little sensibility; but when in a state of inflammation they are extremely sensitive and painful.

Chemic analysis shows that bone consists of organic and inorganic matter; the organic is called animal, the inorganic, earthy material; these are intimately combined; the animal matter furnishes elasticity and toughness, the earthy, hardness and solidity.

How can we separate the two kinds of tissues to prove that this is the case? This is done by steeping a portion of bone in dilute nitric or hydrochloric acid: in this way the earthy material is taken up, leaving the animal or organic material behind, so that the bone can be twisted into any shape, thus demonstrating its elasticity and toughness. The presence of earthy material may be demonstrated by 
subjecting the bone to strong heat in an open fire where the air can have free access, until all the organic material is consumed, when the earthy parts will be found to be brittle, preserving the original shape of the bone.

The organic constituents of bone make up one-third, or 33.3 per cent., of the whole; the inorganic matter, twothirds, or 66.7 per cent. For the convenience of the nurse we append the following table of analysis by Berzelius (from Wilson):

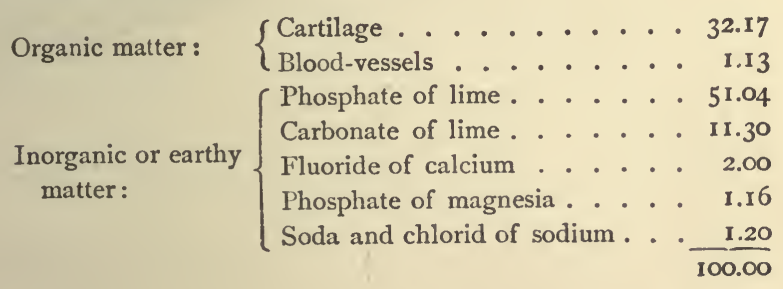

Some difference exists in the proportion of the two constituents of bone at different periods of life. Thus in a child the animal matter predominates, whereas in the aged the bones contain a larger proportion of earthy matter, the animal matter being deficient in quantity and of impaired quality. Hence in children it is not uncommon to find, after an injury, that the bones become bent or partially broken-a condition known as greenstick fracture-whereas in old persons the bones are more brittle and fracture thus takes place more readily. Many of the diseases, also, to which bones are liable are due to a disproportion between the two constituents of bone. Thus in the disease known as rickets, so common among children of the poor, the bones become bent or curved, either from the superincumbent weight of the body or under the action of certain muscles. This is due to some defect of nutrition by which bones are deprived of their 
normal proportion of earthy matter, whereas the animal matter is of unhealthy quality.

Cartilage is a non-vascular structure which is found in various parts of the body -in adult life chiefly in the joints, in the parietes of the thorax, and in various tubes, such as air-passages, nostrils, and ears, which are to be kept permanently open. In the fetus, at an early period, the greater part of the skeleton is cartilaginous. As this fetal cartilage is afterward replaced by bones, it is called "temporary," in contradistinction to that which remains unossified during the whole of life, and which is called "permanent." The first forms the original framework of the skeleton, and becomes ossified. Permanent cartilage is not prone to ossification.

Cartilages are classified, according to their function and position, into articular, interarticular, costal, and membraniform. Cartilage is divided, as to its minute structure, into true or hyaline cartilage, fibrous or fibrocartilage, and yellow, elastic, or reticular cartilage.

Articular cartilage forms a thin layer upon the joint-surfaces of the bones, and its elasticity enables it to break the force of any concussion, while the smoothness affords ease and freedom of movement. It varies in thickness according to the shape of the bone on which it lies. A cartilage derives its nutriment partly from the vessels of the neighboring synovial membrane as well as from the bones upon which they are implanted.

Marrow.-The marrow is a complex tissue which fills up the cylindric cavity in the shafts of the long bones; it also occupies the spaces of the cancellous tissue and extends into the larger bony canals (Haversian canals) which contain the blood-vessels. It is of two colors, yellow and red; the former is found in the long bones of the adult, and is composed of fat 96 per cent., areolar tissue and vessels I per cent., fluid 3 per cent., with ex- 
tractive matter. In the flat and short bones, in the articular ends of long bones, in the bodies of the vertebra, in the cranial diploë, and in the sternum and ribs it is of red color, and contains 75 per cent. water, 25 per cent. of solid matter, consisting of albumin, fibrin, extractive matter, salts, and a mere trace of fat. Marrow possesses five kinds of cells: fat cells, marrow cells proper, resembling leukocytes, nucleated red cells, cells containing red corpuscles, and giant cells.

Vessels of Bone.-The blood-vessels of bone are very numerous. Those of the compact tissue are derived from a close and dense network of vessels ramifying in the periosteum. From this membrane vessels pass into the minute orifices in the compact tissue, running through the canals which traverse its substance. The cancellous tissue is supplied in a similar way with a less numerous set of larger vessels. In the long bones numerous apertures may be seen at the ends near the articular surfaces, which give passage to the vessels referred to; but the most numerous and largest apertures are for the veins of the cancellous tissue, which run separately from the arteries. The medullary tissue of all long bones receive a good-sized artery (the medullary artery), which obliquely penetrates the compact tissue at the nutrient foramen, after which it divides into two main branches, one ascending, the other descending, in the medullary canal; the veins chiefly emerge through numerous openings near the articular ends of the bones, and form the nutrient foramen. Nerves are distributed freely to the periosteum, and accompany the nutrient arteries into the interior of the bone.

Haversian Canals. - The Haversian canals run parallel with the longtiudinal axis of the bone for a short distance, and then branch off and communicate with others. As seen in cross-section in Fig. I they appear 
as round or oval holes, which vary in diameter from $\frac{1}{200}$ to $\frac{1}{500}$ inch. Each canal, as a rule, contains two bloodvessels, a small artery, and vein; the larger ones also contain a small quantity of connective tissue as well as bone cells. The canals near the surface open upon it by minute orifices, and those near the medullary cavity open in the same way into the spaces, so that the whole of the bone is permeated by a system of blood-vessels running through the bony canals in the center of the Haversian system.

Periosteum.-The periosteum is a fibrous vascular membrane which adheres to the surface of the bones in nearly every part, except their cartilaginous extremities. Where strong tendons or ligaments are attached to the bone, the periosteum is incorporated with them. It is composed of two layers closely united together. In young bones the periosteum is thick and very vascular, and is intimately connected at either end of the bone with the epiphyseal cartilages, but less closely with the shaft, from which it is separated by a layer of soft blastema, containing a number of granular corpuscles or "osteoblasts," in which ossification proceeds on the exterior of the young bone. Later in life the periosteum is thinner, less vascular, and the osteoblasts have become converted into a connective-tissue layer, which is separated from the rest of the periosteum in many places by cleft-like spaces, which serve as a nidus for the ramification of the vessels previous to their distribution in the bone; hence the liability of bone to exfoliation or necrosis when denuded of this membrane by injury or disease. Nerves and lymphatics generally accompany the arteries in the periosteum.

Endosteum.-The endosteum is a delicate connectivetissue membrane lining the medullary and cancellous cavities of the bone which contains numerous bone- 
forming cells. Its function is the same as that of the periosteum.

The Development of Bone.-It is customary to describe three varieties of bone development. These are: "Intracartilaginous, intramembranous, and subperiosteal." It should be borre in mind, however, that the essential nature of the process is the same in all. The two latter are considered under one head.

At an early period of life most of the long and irregularshaped bones in the body consist of masses of hyaline cartilage, which present, in a general way, the shape of the future bone. The transformation of these cartilage masses into bone is intracartilaginous ossification. This is always associated with a certain amount of subperiosteal ossification. The bones of the vertex of the skull and those of the face, with few exceptions, are formed in membrane; the base of the skull, the body, and the limbs are formed in cartilage. In the former the bone-forming cells (osteoblasts) arrange themselves along the thick bundles of fibrous tissue which radiate from the center of the future bone; by the deposit of lime-salts in these osteoblasts bone-tissue grows out in needle-like rays toward the circumference. A deposit of bone begins at one spot in the cartilage and is termed the primary center. The shaft is formed from this. Just before ossification commences the cartilage cells enlarge and arrange themselves in rows. Lime-salts are deposited in the matrix between the rows, forming columns which enclose oblong spaces-the primary areolæ-containing cartilage cells. Ossification proper now commences by the ingrowth from the periosteum of buds of young connective tissue, covered with bone-forming cells, which, after causing absorption of the cartilage, become converted into bone. 


\section{CLASSIFICATION OF BONES}

The bones of the body are divided into four groups: long, short, flat, and irregular. These go to make up the frame that protects the internal organs, and provides attachment for the several soft parts.

The long bones are those that make up the extremities; the short bones are those of the hands and feet; the flat bones are those of the shoulder-blades, pelvis, skull, etc.; the irregular bones are those of the spine, as well as the ethmoid, the temporal, etc.

What have we to say about the surfaces of bones? If you examine a bone, you will notice that it has certain eminences and depressions; these have technical names, and it should be the aim of the nurse to become acquainted with each, so that she will be able to describe a part concerned in an injury in a practical manner. These bony eminences and depressions are of two kinds: articular and non-articular.

Examples of articular eminences are found in the head of the arm bone (humerus) and thigh bone (femur). As examples of articular depressions may be mentioned the glenoid cavity of the scapula (shoulder-joint) and the acetabulum, the cavity with which the thigh bone articulates with the pelvis.

Non-articular eminences are distinguished by their form; for example, a broad, rough, uneven part of a bone is called a tuberosity; a small, rough prominence, a tubercle; a sharp, slender, pointed eminence, a spine; a narrow, rough elevation, a ridge or a line. The non-articular eminences and depressions serve to form a larger field for muscular attachments, and are greater in persons who are well developed from exercise.

Non-articular depressions vary in form, and are known as fossæ, grooves, furrows, fissures, notches, etc. 


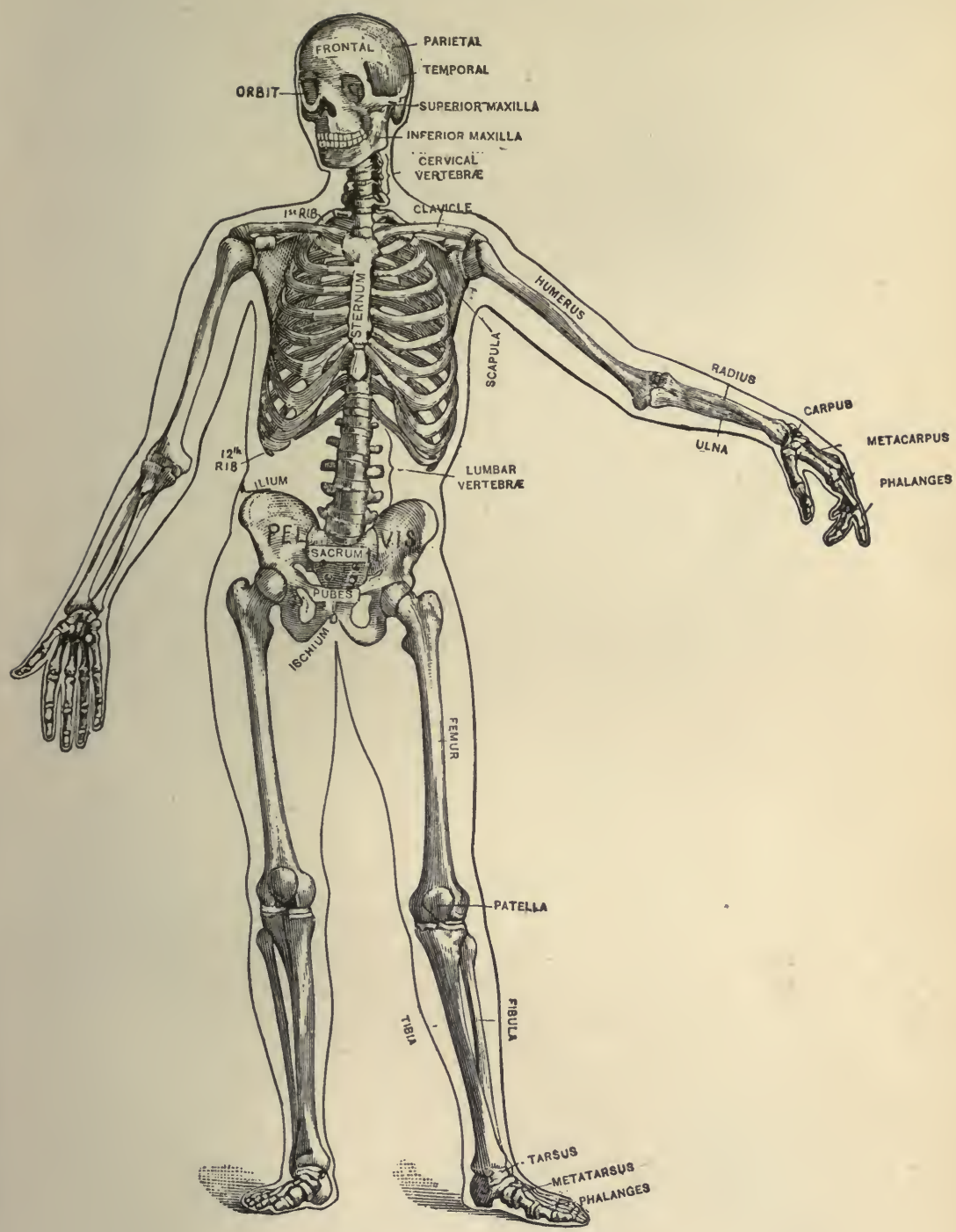

FIG. 3.-The human skeleton. 
The entire skeleton of the adult is made up of 200 distinct bones. These are divided, according to Gray, as follows:

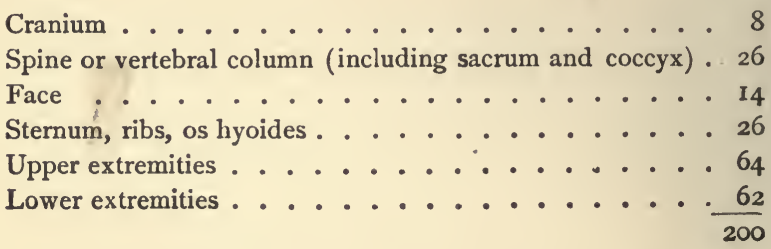

This classification includes the patellæ, but not the smaller sesamoid bones nor the small bones of the ear. The sesamoid bones are found in the substance of the tendons, especially of the great toe. Wormian bones are found in the cranial sutures of childhood; they are irregular fragments, developed from supplementary centers, ultimately closing the fontanel.

\section{THE SPINAL COLUMN}

The spine is a flexible column, formed of a series of bones called vertebræ. These 33 bones are divided into five regions, named, according to their location, as follows: The cervical, consisting of 7 bones; dorsal, 12 bones; lumbar, 5 bones; sacral, 5 bones; coccygeal, 4 bones. At a period of life varying between the ages of twenty-five and thirty years the bones in the sacral and coccygeal regions become united and form 2 bones.

The vertebræ vary in size and in shape. They are placed one upon the other, thus forming a support for the head and trunk. Each vertebra is divided into two parts, the anterior forming the body, the posterior, the arch; the arch is formed by 2 pedicles and 2 laminæ, supporting 7 processes-viz., 4 articular, 2 transverse, and I spinous process. The functions of the processes are to give at- 
tachment to the various muscles, to act as levers to move

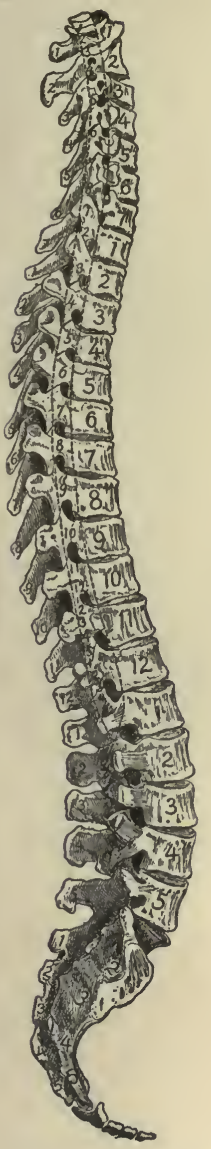

FIG. 4-The figures indicate the relations of the vertebral bodies and spines to the corresponding spinal segments of the cord (Church). the spine in different directions, and to articulate with neighboring bones. The arches of the vertebræ form a canal for the passage of the spinal cord; between each pair of vertebræ apertures exist through which the spinal nerves pass from the cord.

The atlas supports the head; the axis articulates with the atlas; each of the I 2 dorsal segments articulates with the ribs of each side; the sacrum articulates with the two hip-bones (ossa innominata) and the last lumbar; the $\operatorname{cocc} y x$ articulates with the last sacral. The average length of the spine is about 2 feet 4 inches. It presents several curves that correspond to the dif-

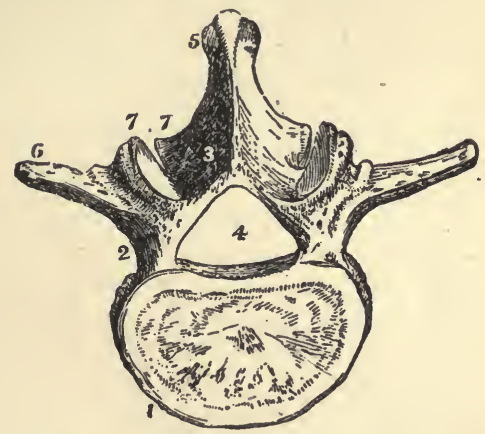

FIG. 5.-A type-vertebra: I, body ; 2, pedicle; 3 , lamina; 4 , spinal foramen ; 5 , spinous process; 6 , transverse process; 7 , articular process (Leidy).

ferent segments of the column, viz.: the cervical with 
the neck; the dorsal with the chest or thorax; the lumbar with the abdomen; the sacral with the pelvis.

The nurse should carefully study the several illustrations in order fully to understand the shape of the segments that form the spine, as each has important connections.

The physician will frequently direct that a nurse apply a blister or a hot application to one of the several regions, and she must, therefore, be well informed in this respect in order that his directions may be intelligently carried out.

\section{THE SKULL}

The skull is considered as an expansion of the vertebral column. It is the bony framework of the head, and contains a cavity for the reception of the brain, vessels, nerves, and membranes. The bones of the skull are divided into two classes - the cranial consisting of 8 bones, and the facial consisting of 14 bones. The nurse should be familiar with the names and location of these bones, in order that she may be able to describe to the physician the exact location of pain, and, in surgical cases, to make accurate records during the physician's absence. The following bones enter into the formation of the skull:

$\begin{aligned} \text { Bones of the cranium : } & \begin{cases}\text { I } & \text { occipital. } \\ 2 & \text { parietal. } \\ \text { I } & \text { frontal. } \\ 2 & \text { temporal. } \\ \text { I } & \text { sphenoid. } \\ \text { I } & \text { ethmoid. }\end{cases} \\ \text { Bones of the face: } & \begin{cases}2 & \text { nasal. } \\ 2 & \text { superior maxillary. } \\ 2 & \text { lacrimal. } \\ 2 & \text { malar. } \\ 2 & \text { palate. } \\ 2 & \text { inferior turbinated. } \\ 1 & \text { vomer. } \\ \text { I } & \text { inferior maxillary. }\end{cases} \end{aligned}$


The occipital bone forms the back and base of the cranium and articulates with the atlas. It contains the foramen magnum, an opening for the exit of the spinal cord.

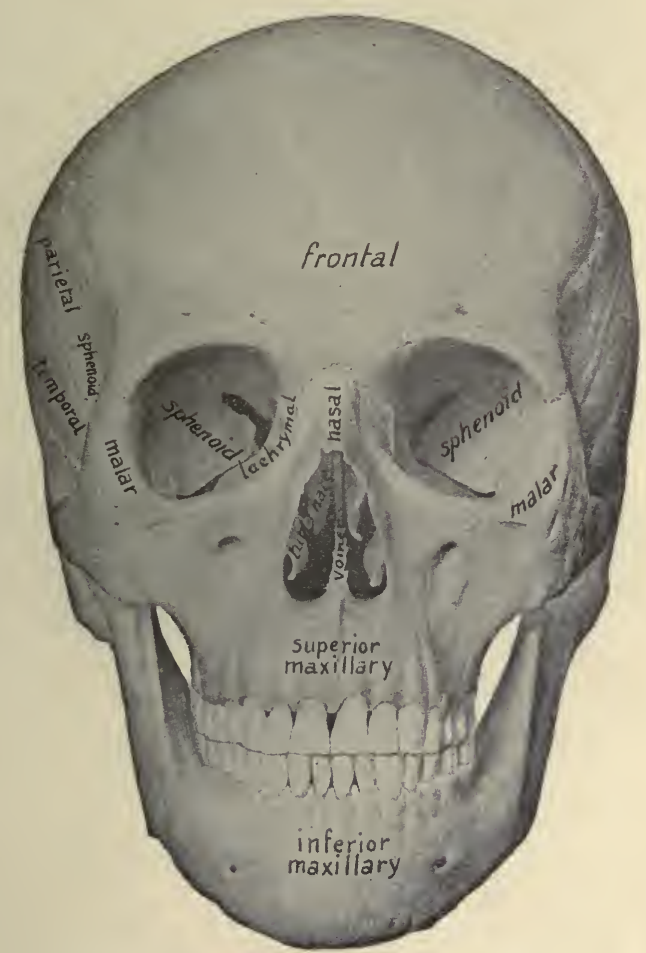

FIG. 6.-Front view of the skull (Sobotta).:

The parietal bones form the sides and roof of the cranium. The frontal bone forms the forehead, and is often the seat of pain in diseases of the head and eye, as it forms part of the bony cavity that receives the eye. It also unites with the two parietal bones, thus forming the space in the center of the head called the anterior 
fontanel, and generally known among mothers as the

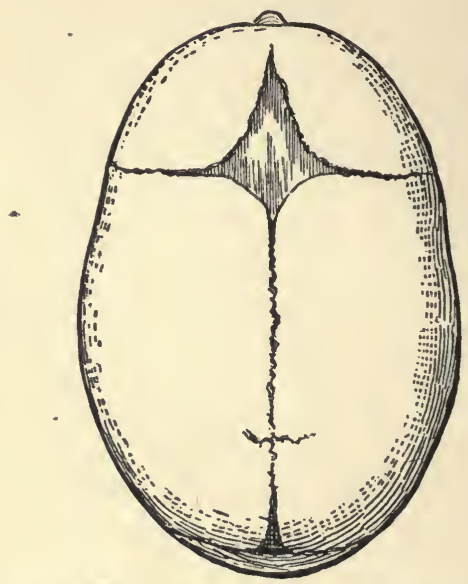

FiG. 7.-Infant's skull: anterior fontanel above; posterior fontanel below (J. P. C. Griffith).

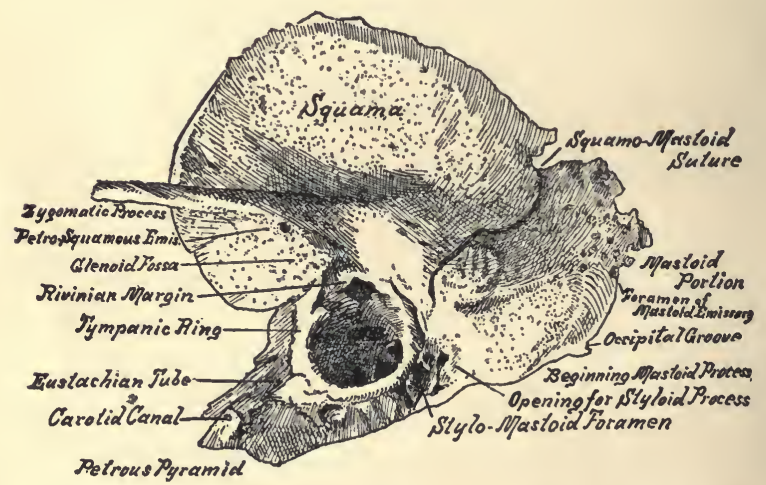

FIG. 8.-Temporal bone of infant; lower outer surface, showing squamous, tympanic, and petromastoid segments (Randall).

"soft spot"; this is found in the infant at birth, but ultimately becomes obliterated. 
The temporal bones are situated at the side of the head, and contain the organs of hearing. A knowledge of the location of these bones is important, as they are frequently the seat of disease; they should, therefore, be well considered by the nurse. In children, especially after scarlet fever, these bones often become diseased and require the attention of the nurse; such disease may result in chronic discharges.

The sphenoid and ethmoid bones are situated at the base of the skull, and form the connection between all the

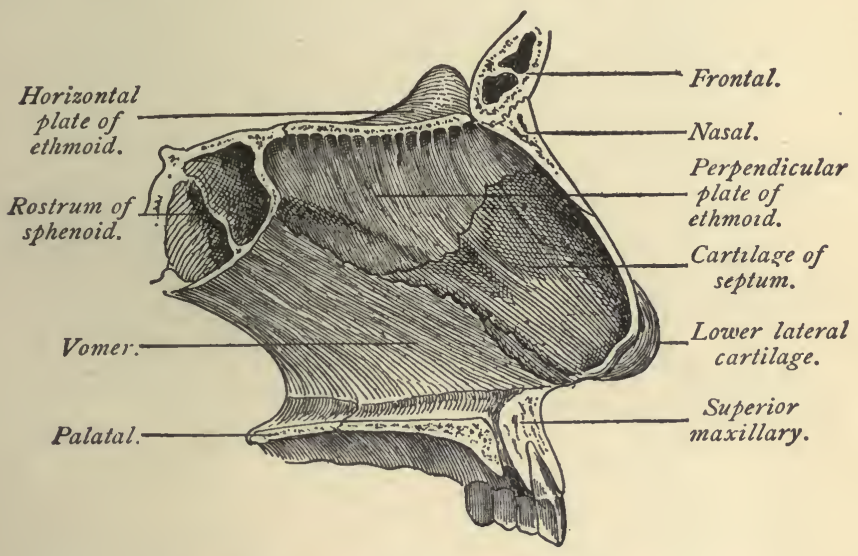

FIG. 9.-Cartilage and bones of the septum of the nose (Ingals).

bones of the cranium and face, with the exception of the inferior maxillary.

The two nasal bones form the bridge of the nose; the vomer, the back part of the septum.

The superior maxillary bones are the largest bones of the face, excepting the inferior maxillary, and contain.sockets for the reception of the upper teeth. They aid in forming three cavities in the head-the roof of the mouth, the floor and outer wall of the nose, and the floor 
of the orbit, which receives the eye. There is also within each bone a cavity, called the antrum of Highmore, which in some subjects becomes the seat of a serious inflammation; this cavity is situated at about the middle of the bone, and renders the bone lighter.

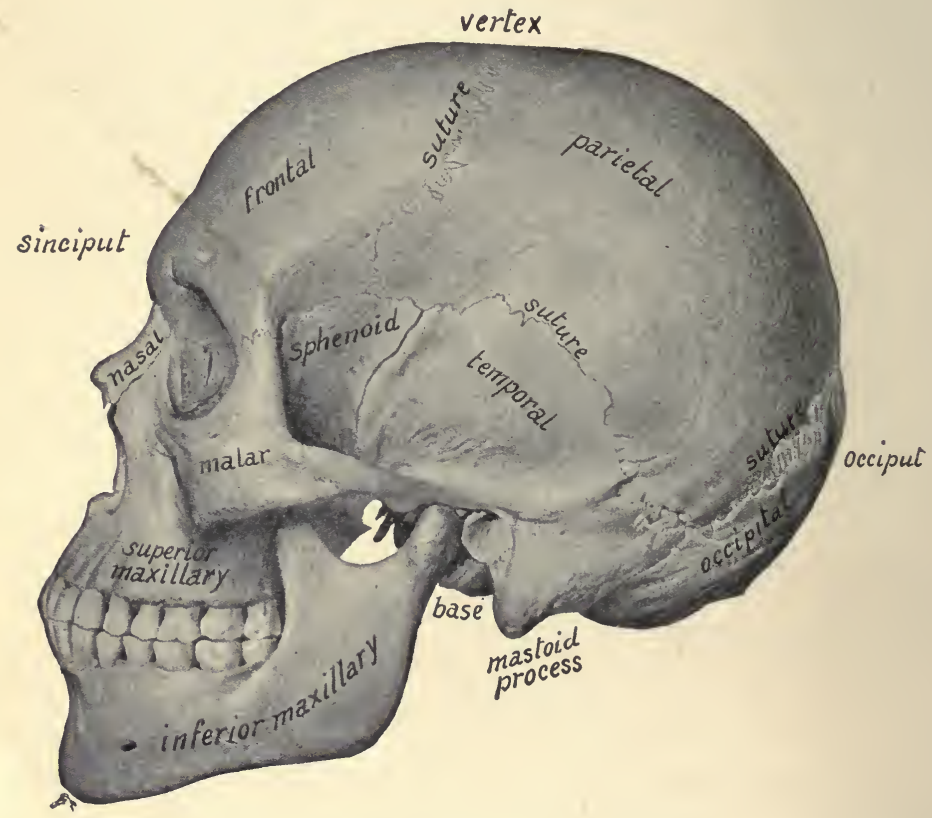

FIG. Io.-Side view of the skull (Sobotta).

The malar bones go to make up the prominence of the cheek, and assist in forming the cavity for the reception of the eye. The lower part of the posterior border articulates with the temporal bone to form the zygomatic arch.

The palate bones are situated at the back part of the nose, helping to form the nasal, oral, and orbital 
cavities. The inferior turbinated bones are found on the outer wall of the nasal cavity. The vomer forms the central septum of the nose.

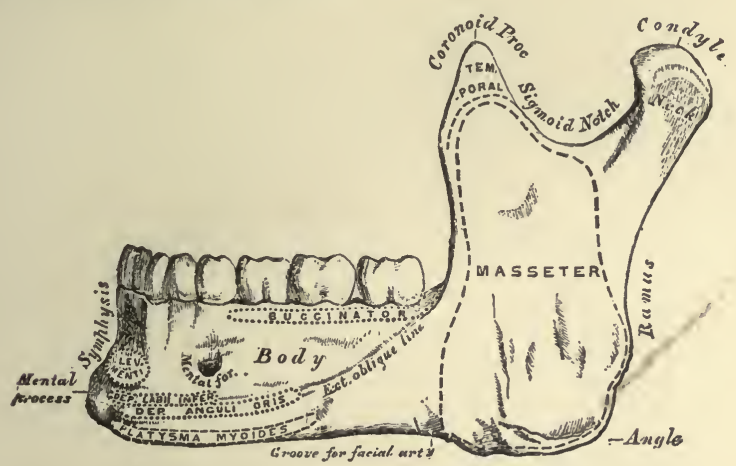

FIG. II.-Inferior maxillary bone, outer surface, side view (Gray).

The orbit is the cavity for the reception of the eye, and is formed by seven bones.

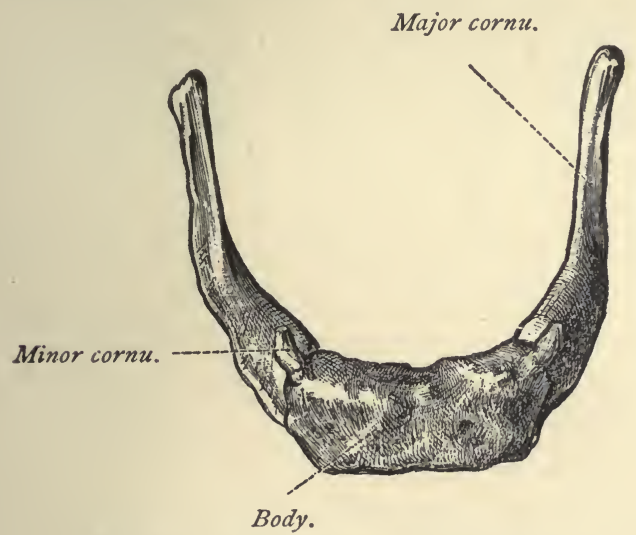

FIG. I2.-The hyoid bone, viewed from above (Toldt).

The inferior maxillary bone consists of a body, with two rami, which are surmounted on either side by a 
thin triangular coronoid process in front, and an articular condyloid process posteriorly. The constricted portion beneath the condyle is termed the neck of the condyle. The depression separating these two processes is called the sigmoid notch, and is crossed by the mesoteric vessels and nerve. This bone is one with whose location the nurse should be familiar, as it is subject to many conditions that require surgical attention. It is most liable to fracture, and frequently becomes dislocated as the result of a very light blow. It contains sockets for the reception of the lower teeth.

The hyoid bone, sometimes called the lingual bone, is a bony arch shaped like a horseshoe. It supports the tongue and gives attachment to numerous muscles.

\section{THORAX AND PELVIS}

The thorax is that part of the body that goes to make up the chest. It forms a protection for the organs of respiration and circulation, inclosing one of the cavities of the trunk, known as the thoracic cavity. The thorax is conic in form, and its osseocartilaginous framework is formed by the thoracic vertebral bodies behind, the ribs laterally, and the costal cartilages and sternum in front; its base is formed by the diaphragm. Through its apex pass the great cervical vessels, the pneumogastric, phrenic and sympathetic nerves, the trachea, esophagus, thoracic duct, and apices of the lungs covered by pleura.

The sternum, popularly known as the breast-bone, is divided into three parts - the manubrium, or handle; the gladiolus, or sword; and the ensiform, or xiphoid appendix. The manubrium articulates with the clavicle and first rib; the gladiolus with cartilages of part of the second, all of the third to sixth, and part of the seventh ribs on each side; the ensiform articulates with part of the seventh costal cartilage and cartilages of the false ribs. 
There are twelve ribs on each side; these are of different shapes, conforming to their location. 'They serve for the attachment of the several muscles of the chest and abdomen. They are all connected behind with the dorsal vertebræ of the spine, and the first seven are connected with the sternum by the costal cartilages; these are called true ribs. The remaining five pairs are called false ribs;

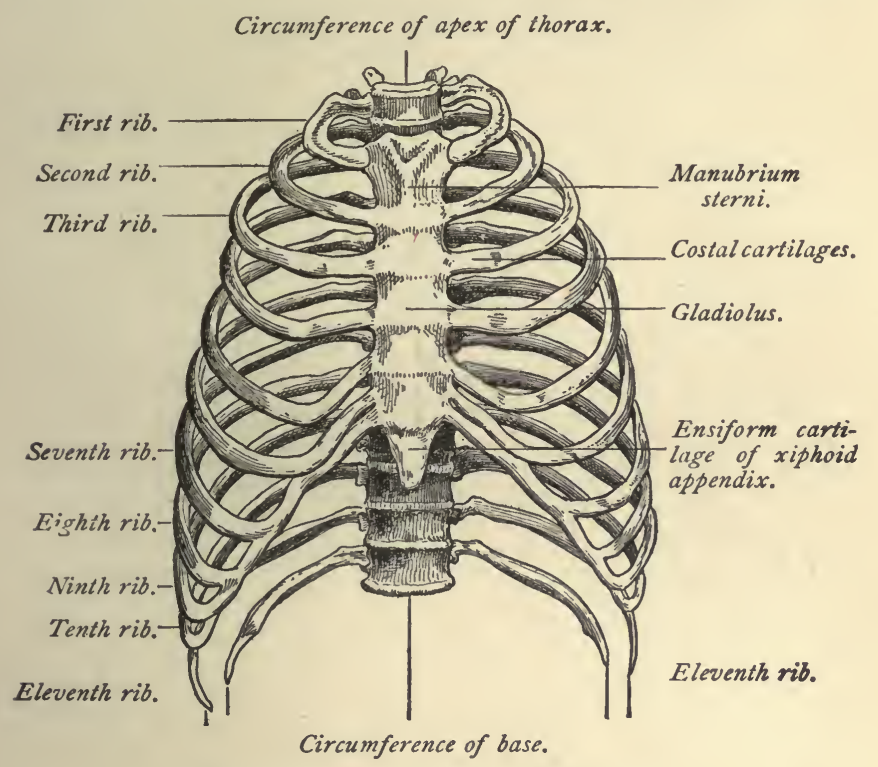

FIG. 13.-Thorax (anterior view) (Ingals).

of these, the first three are attached in front to the costal cartilages, and are termed the vertebrocostal, while the remaining two, being unattached in front, are known as vertebral or floating ribs.

The convexity of each curved rib is turned outward, so as to give roundness to the side of the chest and increase the dimensions of its cavity when the ribs are raised; 
each slopes downward from its vertebral attachment, so that its sternal end is considerably lower than its dorsal.

The ribs are classed as irregular bones. The spaces between the ribs are calied intercostal spaces, a fact to be remembered by the nurse when describing the location of pain or of an eruption.

Below the diaphragm, and above the pelvic bones, we have the second cavity of the trunk, called the abdominal

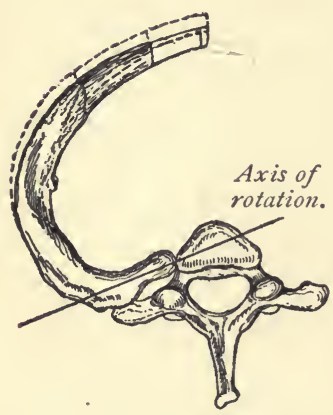

FIG. I4.-First dorsal vertebra and rib (Reichert).

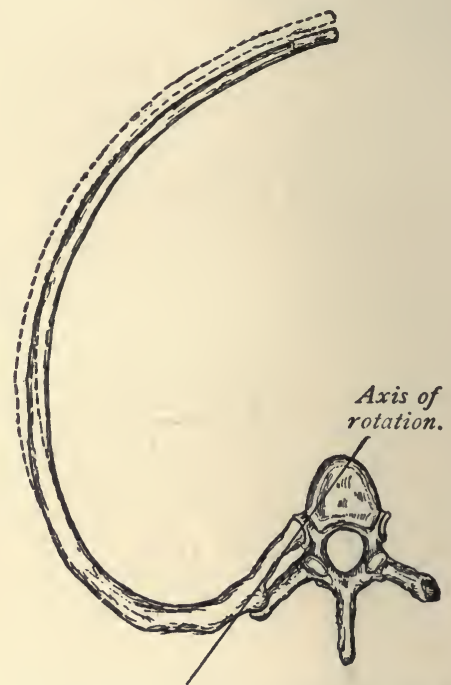

FIG. 15.-Sixth dorsal vertebra and rib (Reichert).

\%

cavity; this contains the organs of digestion, which will be considered when discussing visceral anatomy.

The pelvis is a basin-like cavity, formed by the sacrum, coccyx, and two ossa innominata. It is divided into two parts by the brim of the pelvis or pelvic inlet. The portion of the pelvic cavity above the pelvic brim is a part of the abdominal cavity; the portion below is the true 
pelvic cavity. The latter contains the organs of generation, the rectum, the bladder, the ureters, and the blood-vessels. The pelvic outlet is formed by the pubic arch in front and the coccyx and sacrosciatic ligaments behind; the lateral boundaries are formed by the tuberosities of the ischia. The transverse diameter of the pelvic outlet averages $3 \frac{1}{2}$ inches in the male and $4 \frac{3}{4}$ inches in the female.

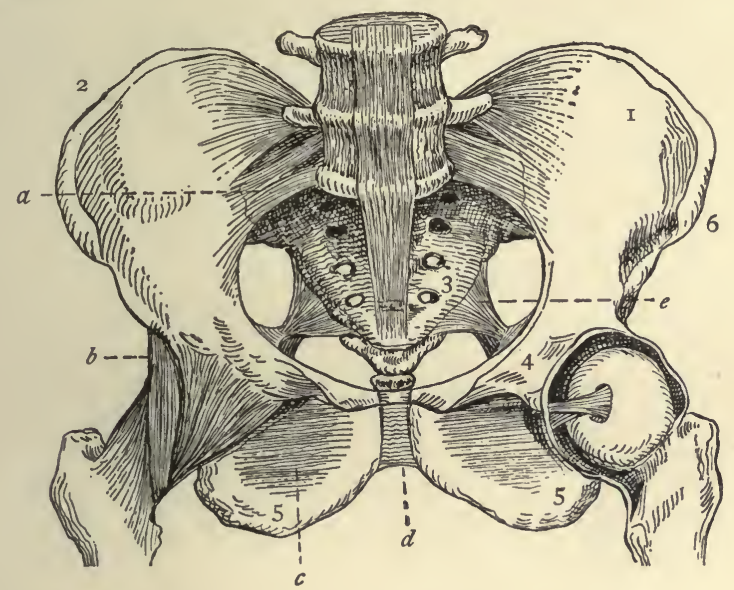

FIG. I6.-Front view of the pelvis, with its ligaments: I, innominate bone; 2 , crest of ilium; 3 , sacrum; 4 , pubes; 5 , tuberosity of ischium; 6 , anterior superior spine of ilium; $a$, anterior sacroiliac ligament; $b$, iliofemoral ligament; $c$, obturator membrane; $d$, symphysis pubis; $e_{1}$ sacrosciatic ligament (Dorland).

The sacrum is a curved, triangular bone, situated between the two ossa innominata, and forming, with the coccyx, the posterior part of the pelvis. It is formed of five vertebræ, which, after the twenty-fifth year, become united as one bone. The coccyx consists of four bones articulating with the sacrum, and is subject to fractures and necrosis, conditions that require surgical operations for their cure. Injuries to this bone sometimes occur dur- 
ing labor, especially in instrumental cases, from the pressure of the child's head in its passage through the outlet of the pelvis.

The ossa innominata, or nameless bones, form the sides and anterior wall of the pelvic cavity. They are divided into three parts - the ilium, the ischium, and the pubes; in adults these become consolidated and form one bone. At the junction of the three parts is found the acetabulum, which articulates with the head of the femur. The upper border of the brone is called the crest of the ilium; the lower, the ischium; and the anterior, the pubes. The anterior extremity of the iliac crest is called the anterior superior spine of the ilium; that portion of the ischium upon which we sit is the tuberosity; the junction of the pubic bones is known as the symphysis. These are important landmarks.

\section{THE BONES OF THE UPPER EXTREMITY}

The bones of the upper extremity are the clavicle and scapula (comprising the shoulder girdle), the humerus, the radius and ulna, the carpal bones, the metacarpal bones, and the phalanges.

- The clavicle or collar-bone, is shaped like the letter $f$, and articulates with the sternum and the scapula.

It has a wide range of motion, and this, together with its exposed position and the fact that it is the only bony connection between the trunk and the upper extremity, explains why the clavicle is more frequently broken than any other single bone in the body.

The scapula or shoulder-blade, is situated on the posterior and lateral part of the thorax, overlying the region included between the second and the seventh ribs. The dorsal surface of the bone is traversed by an elevated ridge- the spine - the outer extremity of which overhangs the shoulder, and is known as the acromion. Just beneath 


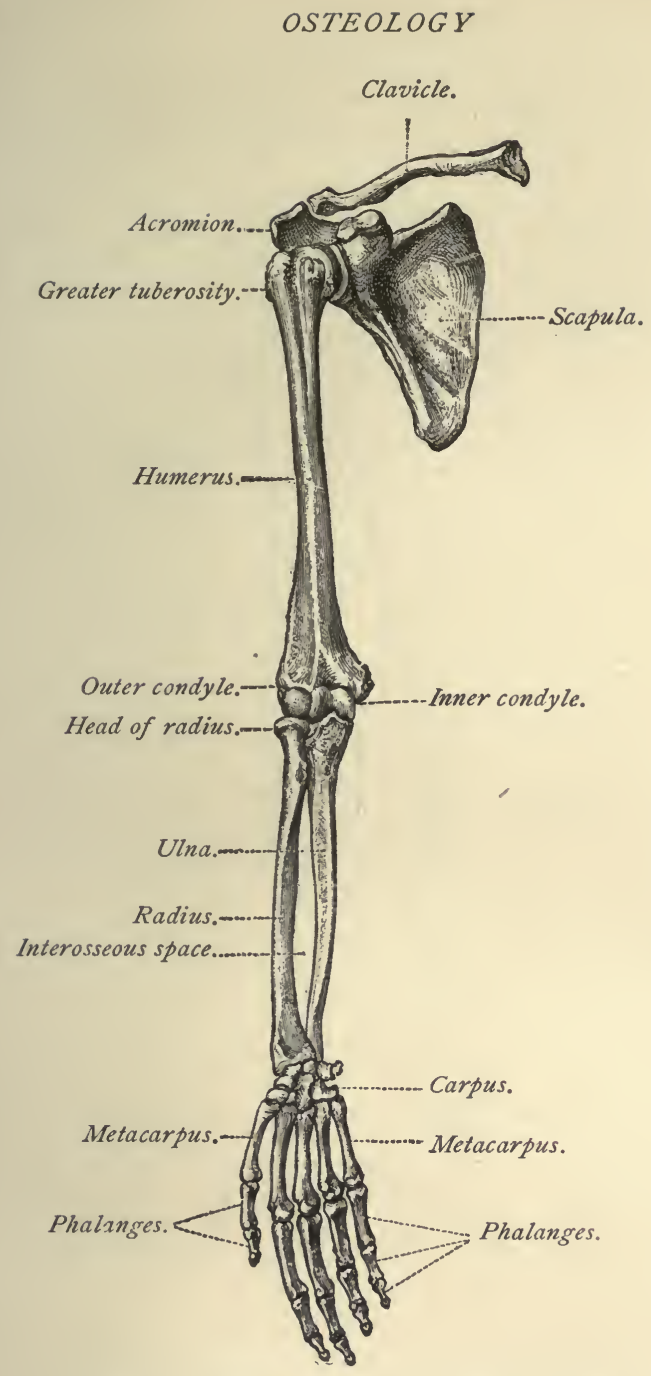

FIG. 17.-Bones of the upper extremity (Toldt).

the acromion process is found a shallow depression-the glenoid cavity-which articulates with the head of the 
humerus. The coracoid process (or "crow's beak") is situated to the inner side of the glenoid cavity and gives

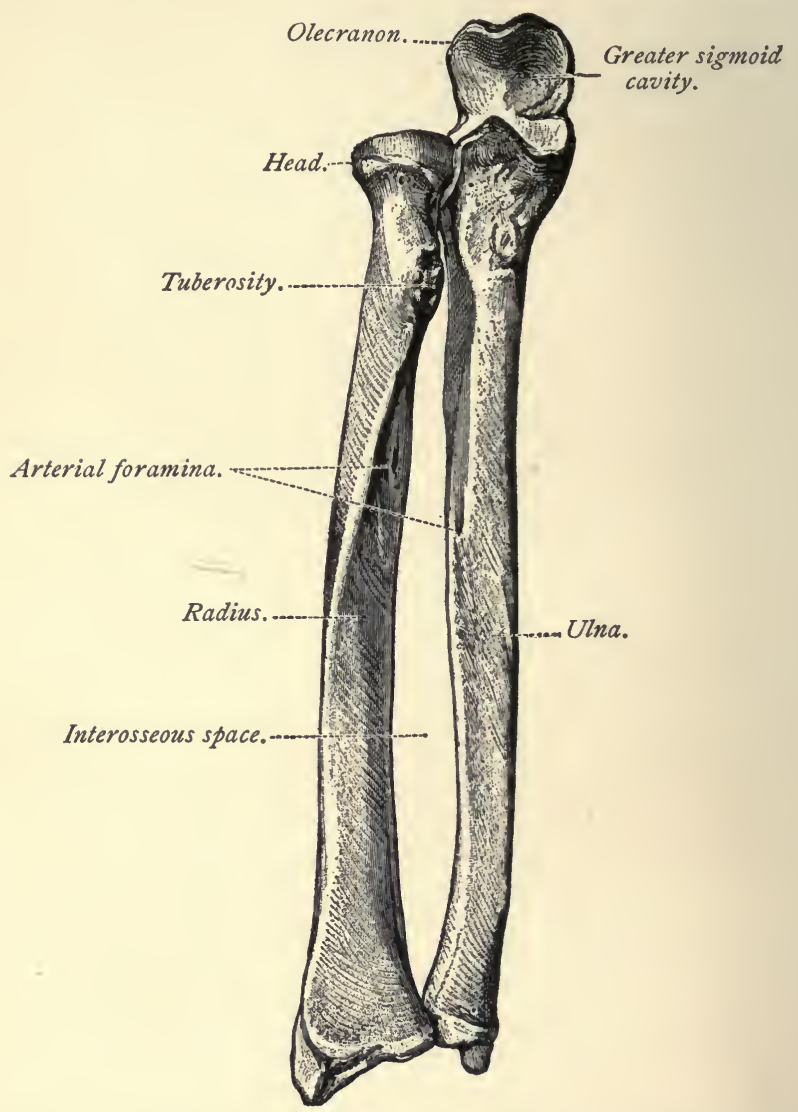

FIG. I8.-Bones of the right forearm in a position of supination (Toldt).

origin to a portion of the biceps muscle. The scapula is classified as a flat bone.

From the standpoint of the anatomist, that portion of the upper extremity between the shoulder and the elbow 
is the arm, while that between the elbow and wrist is the forearm.

The humerus is the long bone in the arm. Its upper extremity presents for examination a rounded head, which articulates with the glenoid cavity of the scapula, and two eminences, the greater and the lesser tuberosity. "The circumference of the articular surface of the head is known as the anatomic neck. Since the constricted portion of the bone below the tuberosities is frequently fractured, it is called the surgical neck. The lower expanded extremity of the humerus is flattened from before backward to form the condyles. The internal condyle articulates with the ulna, while the external articulates with the radius.

The ulna is the inner bone of the forearm; it is longer than the radius, and helps to form the elbow-joint, but does not enter into the formation of the wrist-joint. The upper end presents a curved process, called the olecranon, and the cavity thus formed articulates with the humerus, and is called the sigmoid cavity.

The radius is the outer bone of the forearm, and is shorter than the ulna. The upper rounded head helps . to form the elbow-joint; the lower end articulates with the carpus to form the wrist-joint, and is subject to many injuries, the most common of which is that known as "Colles' fracture." Many suits for malpractice have been instituted as the result of this injury, the physician often being held responsible for bad results that followed carelessness on the part of the patient.

When the radius and ulna are parallel, the foramen is said to be supinated; when the bones are crossed, the forearm is pronated.

THE HAND

The bony framework of the hand is made up of the carpus, which consists of eight bones, the metacarpus, 
consisting of five bones, and the fourteen bones termed the phalanges. The bones of the carpus, together with the radius, enter into the formation of the wrist-joint. The bones of the upper row are the scaphoid, semilunar, cuneiform, and pisiform; those of the lower row, the trapezium, trapezoid, os magnum, and unciform.

The metacarpus forms the palm of the hand. The phalanges form the fingers; each finger is made up of three phalanges, the thumb, of two. Although classified

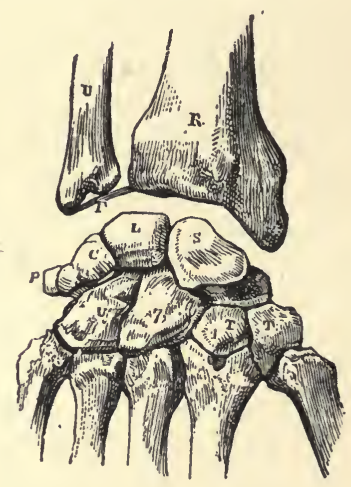

FIG. I9.-Right carpal bones, dorsal surface: S, scaphoid; L, semilunar; C, cuneiform; P, pisiform; U, unciform; 7 , os magnum ; ${ }_{1} \mathrm{~T}$, trapezoid; T, trapezium (Leidy).

as long bones, they are also numbered among the short bones of the body. Each bone is composed of a base, a shaft, and an extremity.

THE BONES OF THE LOWER EXTREMITY $\Rightarrow, \ldots$

The lower extremity is composed of the thigh, the leg, and the foot.

The femur, or thigh-bone, is the longest bone in the body. The rounded head articulates with the acetabulum of the os innominatum. The head is joined to the shaft 


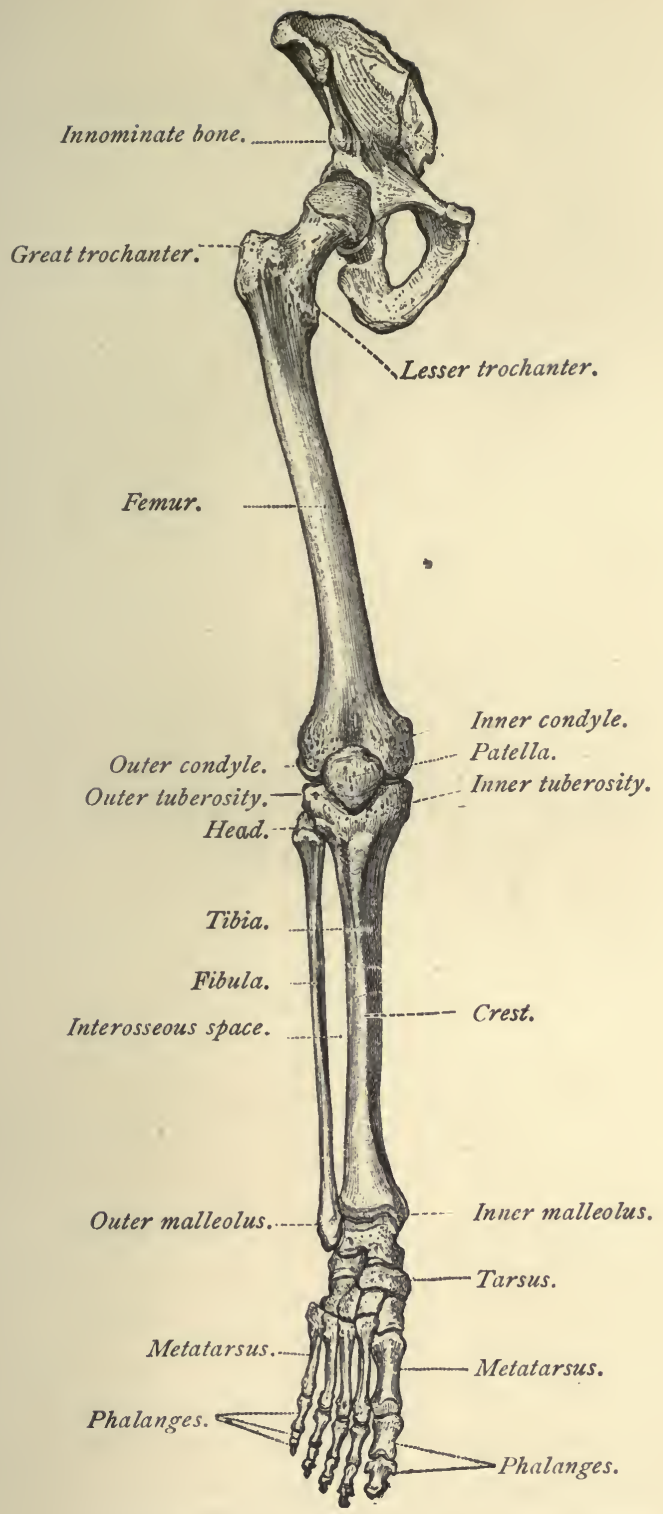

FIG. 20.-Bones of the lower extremity (Toldt). 
by a constricted neck. At the junction of neck and shaft are found the greater and lesser trochanters. The lower end of the bone is expanded to form two large condyles, which articulate with the tibia and the patella. It gives attachment to the strongest muscles of the body.

The leg consists of two bones-the tibia and the fibula. The patella is a sesamoid bone embedded in the tendon of the quadriceps.

The tibia is the large bone on the inner side of the leg. The upper portion is expanded to form two tuberosities which articulate with the condyles of the femur to form the knee-joint. The sharp anterior edge of the bone
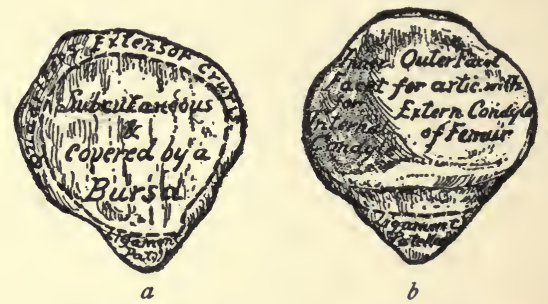

FIG. 2r.-Right patella : $a$, Anterior surface; $b$, posterior surface (Nancrede).

is called the crest ("the shin"). The lower end of the tibia is known as the inner malleolus, and articulates with the astragalus, thus aiding in the formation of the anklejoint.

The fibula is the long, slender bone on the outer side of the leg. The upper end, or head, articulates by a flat facet with the tibia and takes no part in forming the knee-joint. The lower end of the bone is the outer malleolus and helps to form the ankle-joint. A fracture of the lower fifth of the fibula is commonly called a Potts' fracture.

The patella, or knee-cap, is a flat, triangular bone that helps to make up the knee-joint. The lower portion, 
or apex, is connected with the tibia by the tendo patella. The posterior surface of the bone presents two facets which articulate with the condyles of the femur. The anterior surface is separated from the skin by a bursa; when this bursa is enlarged the condition is known as "house-maids' knee."

\section{THE FOOT}

The foot is divided into the tarsus, which consists of 7 bones, the metatarsus, consisting of 5 bones, and the

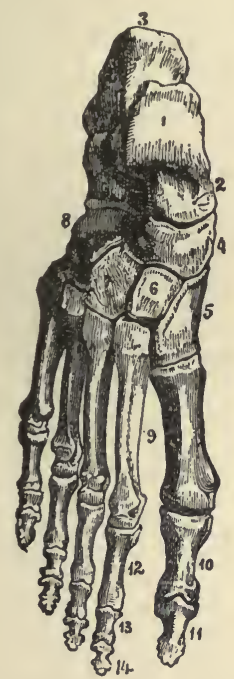

FIG. 22.-Bones of the right foot, dorsal surface : I, Astragalus ; 2, head of the astragalus; 3 , os calcis; 4 , navicular bone; 5 , internal cuneiform; 6 , middle cuneiform; 7 , external cuneiform; 8 , cuboid ; 9 , metatarsal bones ; I0, II, I2, I3, I4, phalangeal bones (Leidy).

phalanges, 14 in number, making a total of 26 bones. They are placed in two rows. The uppermost bone of the tarsus is called the astragalus and articulates with the tibia and fibula. 
The heel-bone is in the lower posterior part of the foot, and is called the os calcis. It is classed as an irregular bone. The 5 metatarsal bones are classed with the long bones and articulate with the tarsus.

The phalanges of the foot number 14, just as in the hand; the great toe is made up of two phalanges, the other toes having three. They are classed as long bones, and, from their location, are subject to many injuries.

\section{THE ARTICULATIONS}

The various bones of which the skeleton is made up are connected at different parts of their surfaces to form joints or articulations. If the joint be immovable,

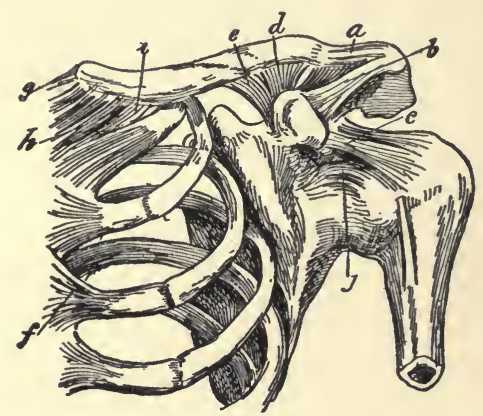

FIG. 23.-Ligaments of the shoulder: $a$, Superior acromioclavicular ligament; $b$, coraco-acromial ligament; $c$, coracohumeral ligament; $d$, transverse ligament; $e$, coracoclavicular ligament; $f$, anterior costosternal ligament ; $g$, interclavicular ligament; $h$, anterior sternoclavicular ligament; $i$, costoclavicular ligament $; j$, capsular ligament.

as those between the cranial and most of the facial bones, the adjacent surfaces are brought into close approximation with an intervening thin layer of fibrous membranethe sutural ligament; in certain situations, at the base of the skull, a thin layer of cartilage is interposed.

Where slight movement combined with great strength is required, as in the joints of the spine, the sacro-iliac, 
and the interpubic articulations, the osseous surfaces are united by tough and elastic fibrocartilage; but in the movable joints the bones forming the articulation are
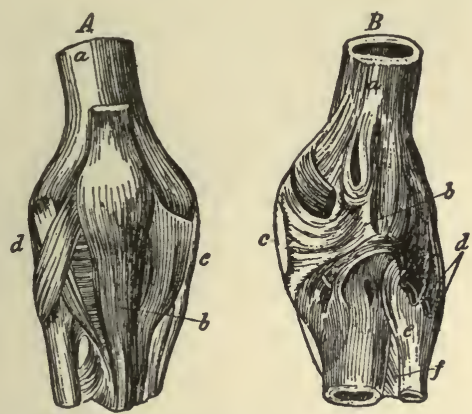

FiG. 24.-Ligaments of the knee-joint: $A$, Anterior aspect: $a$, Femur; $b$, ligamentum patellæ; $c$, internal lateral ligament; $d$, external lateral ligament. $B$, Posterior aspect: $a$, Femur; $b$, posterior ligament ; $c$, internal lateral ligament; $d$, external lateral ligament; $e$, posterior ligament of peroneotibial articulation; $f$, interosseous ligament.
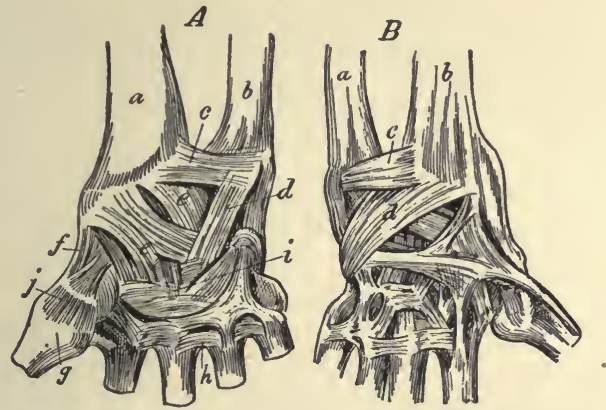

FIG. 25.-Ligaments of the wrist-joint: $A$, Anterior aspect: $a$, Radius; $b$, ulna $; c$, anterior radio-ulnar ligament ; $d$, internal lateral ligament; $e$, anterior ligaments; $f$, external lateral ligaments ; $g$, first metacarpal bone; $h$, palmar ligaments; $i$, palmar carpal ligaments $; j$, capsular ligament. $B$, Dorsal aspect: $a$, Ulna; $b$, radius; $c$, posterior radio-ulnar ligaments ; $d$, posterior ligament.

generally expanded to allow greater freedom for mutual connection. These bones are covered by an elastic structure called cartilage, held together by strong bands or 

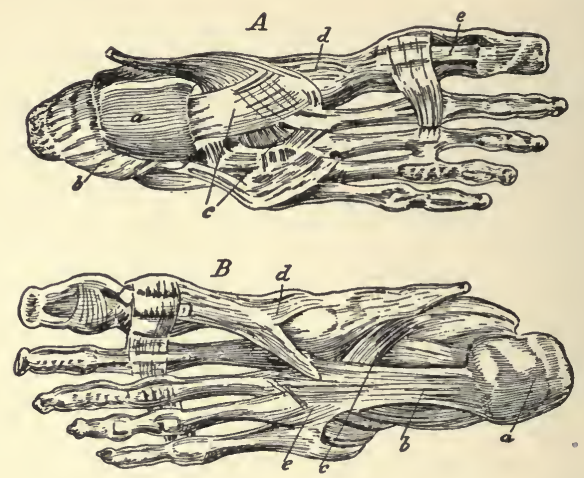

FIG. 26.-Ligaments of the foot: $A$, Dorsal aspect: $a$, Astragalus; $b$, calcaneo-astragaloid ligaments ; $c$, dorsal ligaments of tarsus; $d$, tarsometatarsal ligaments; $e$, transverse ligaments. $B$, Plantar aspect: $a$, Os calcis; $b$, greater calcaneocuboid ligament; $c$, deep calcaneocuboid ligament; $d$, inferior tarsometatarsal ligament; $e$, tarsometatarsal ligaments.

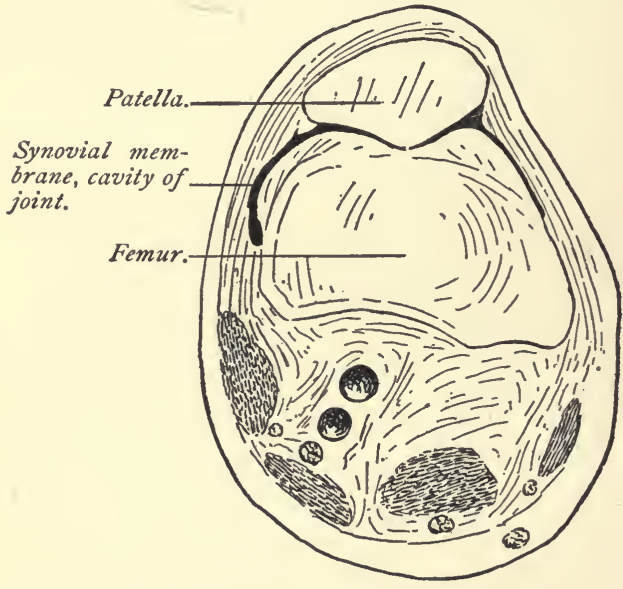

FIG. 27.-Horizontal frozen section of the knee-joint, showing lateral extent of synovial membrane (Professor Dwight's specimen).

capsules of fibrous tissue called ligaments, and lined by a membrane (the synovial membrane) that secretes a fluid that lubricates the various parts of which the joint is 
formed. The structures that enter into the formation of a joint are bone, cartilage, fibrocartilage, ligament, and synovial membrane.

Bone constitutes the fundamental element of all the joints. Cartilage is a firm, opaque, pearly-white, or bluishwhite-in some varieties yellow-highly elastic, flexible tissue, possessed of considerable cohesive power. It yields readily to pressure, and resumes its shape when the force is removed. The synovial membrane will be considered when the membranes of the body are described.

The movable joints of the human body are classified according to their range of motion as follows:

$$
\begin{array}{ll}
\text { Uniaxial joints: } & \left\{\begin{array}{l}
\text { I. Hinge joints. } \\
\text { 2. Pivot joints. }
\end{array}\right. \\
\text { Biaxial joints: } & \left\{\begin{array}{l}
\text { I. Condyloid joints. } \\
\text { 2. Saddle joints. }
\end{array}\right. \\
\text { Polyaxial joints: } & \left\{\begin{array}{l}
\text { I. Gliding joints. } \\
\text { 2. Ball-and-socket joints }
\end{array}\right.
\end{array}
$$

In the hinge joint the motion is about a transverse axis. Examples-the ankle-joint and part of the elbow-joint (the articulation between the humerus and the ulna). In the pivot joint the motion is about a longitudinal axis. Examples - the joint between the atlas and the axis and part of the elbow-joint (the articulation between the ulna and the head of the radius.

In the condyloid joint the two axes of motion are placed at right angles to one another, but both pass through the same bone. Examples - the wrist-joint and the articulation between the atlas and the occiput. The saddle joint is formed by the approximation of two saddle-shaped surfaces, the axes of motion being at right angles to each other, but passing through different bones. Examplethe carpometacarpal joint of the thumb. 
In the gliding joints a spheric head moves in a spheric socket, but the articulating surfaces are segments of such large spheres that they seem to be almost plane. Example - the joints between the articular surfaces of the vertebræ. In the ball-and-socket joints the articular surfaces are more extensive segments of smaller spheres and the capsular ligaments are more roomy, so that a wide range of motion is possible. Examples-the shoulder-joint and the hipjoint.

\section{TENDONS}

Tendons are white, glistening, fibrous cords, varying in length and thickness. They are round, sometimes

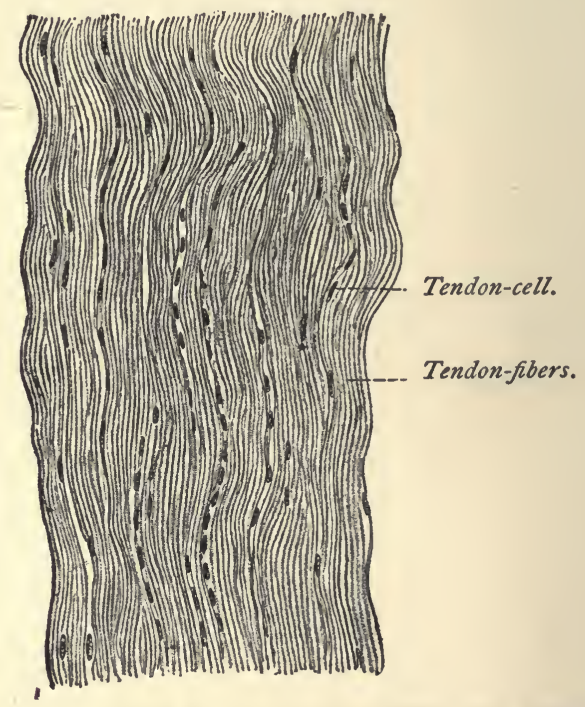

FIG. 28.-Longitudinal section of tendon $(\times 270)$ (Böhm and Davidoff).

flattened, of considerable strength, but slightly elastic, and almost without blood or nerve supply. They serve to connect the muscular tissue to the part to be moved. 
Aponeuroses are membranous expansions of the same tissue and serve a similar purpose.

Fascia are fibrous or fibro-areolar layers investing the soft tissues. The superficial fasciæ are usually fibroareolar, lie beneath the skin, and are loaded with fat; the deep fasciæ resemble aponeuroses. They are dense and inelastic, and either ensheath muscles or serve for their attachment. They tend to preserve the part, since partitions descend between various muscles to become attached to the periosteum.

\section{ADIPOSE TISSUE}

Adipose tissue, or fat, is distributed very generally throughout the body. It has a copious supply of bloodvessels and lymphatics, but no nerves have been known to terminate in it. In some subjects it is present in such abundance about the internal organs as to interfere with their functions. It helps to maintain bodily heat, and rounds out the contour of the body. Its presence or absence is an index of the nutritive and assimilative powers of the individual.

\section{REVIEW QUESTIONS}

Define the term Anatomy.

Into what branches is it divided?

What is Osteology?

What is bone, and what is its composition?

What canals are there in the bone?

What is the periosteum, its function?

Have the bones much sensibility?

State the chemic analysis of bone.

How may the constituents of bone be demonstrated?

Is there any difference in their proportions at different ages?

In what disease is there a disproportion in constituents?

How do fractures in the young differ from fractures in the aged?

Give the reasons for this difference.

Into how many classes are bones divided? Name them. 
Name their location.

What are eminences?

Give an example of same.

What are depressions? Give an example.

How many bones are there in the adult skeleton?

How are they divided as to number?

What are sesamoid bones?

What are Wormian bones, and where are they found?

Mention the several names given to non-articular eminences.

What are non-articular depressions?

What is the spine?

THE SPINAL COLUMN.

How many bones enter into its formation?

Into how many regions is the spine divided?

Give the number in each region.

What is a vertebra?

What are the parts of a vertebra?

What do the several parts form?

What is the function of the processes?

What does the arch form?

What nerves pass through between the vertetræ?

What does the atlas support?

With what bone does the atlas articulate?

What articulates with the dorsal vertebræ?

With what does the sacrum articulate?

With what does the coccyx articulate?

What is the average length of the spine?

What curves does it present?

Of what is the skull considered to be an expansion?

How is it divided?

How many bones in the cranium? The face?

What does the occipital bone form?

What large foramen is found in the occipital bone?

What do the parietal bones form?

What does the frontal bone form?

What is found in the infant at the junction of the frontal and parietal bones?

What important relations have the temporal bones?

Under what circumstances may this bone become diseased?

Where are the sphenoid and ethmoid bones located?

Where are the supericr maxillary bones situated? 
What important cavity is found in this bone?

How many cavities does this bone aid in forming?

Give the location of the malar bones.

What bones make up the orbit?

Where are the palate bones situated?

Where are the turbinated bones found?

What is the vomer, and what does it form?

What does the inferior maxillary bone form?

What is the hyoid bone? Give its location.

THORAX AND PELVIS.

What is the thorax?

What cavity is formed by the thorax?

What bones enter into its formation?

What is the sternum, and how is it divided?

What separates the thoracic from the abdominal cavity?

How many ribs go to make up the thorax?

How are the ribs divided?

To what class of bones do the ribs belong?

What are the spaces between ribs called?

What cavity is found below the diaphragm?

What does this cavity contain?

What do you understand by the pelvis, and what forms the same?

How is the pelvis divided?

What is contained in this cavity?

What forms the outlet?

What is the average transverse diameter of the pelvic outlet?

What is the sacrum?

How many bones constitute the same, and at what age do they consolidate?

What is the coccyx, and to what is it predisposed?

What are the ossa innominata, and what do they form?

Into how many parts are they divided? Name them.

Give the locations of the several parts.

What passes through the openings in this bone?

About what age do these several parts become consolidated?

What is formed by the union of the ossa innominata anteriorly?

BONES OF THE UPPER EXTREMITY.

What bones form the shoulder-girdle?

Describe the clavicle. 
With what bones does it articulate?

What is the scapula?

Where is it located?

What important processes does this bone present?

What cavity is formed by the scapula?

What bone articulates in this cavity?

To what class of bones does the humerus belong?

With what bones does the humerus articulate?

How many bones form the upper extremity?

Which bone is on the inner side of the forearm?

With what does the ulna articulate?

Which is the longest bone of the forearm?

Does the ulna enter into the formation of the wrist-joint?

What curved process is presented by the upper end of the ulna?

What cavity is formed by this process?

What is the radius?

With what does it articulate?

What is the fracture called which occurs at its lower end?

How are the bones of the hand divided?

How many bones constitute each division?

Name the bones in the upper row. The lower.

What part of the hand do the several parts form?

LOWER EXTREMITY.

What is the femur, and with what does it articulate?

c. To what class of bones does the femur belong?

To what does this bone give attachment?

What bones are found in the leg?

Does the patella enter in the formation of the knee-joint?

What is the tibia, and with what bones does it articulate?

What is formed by the lower extremity of the bone?

What is the fibula?

How does this bone articulate with the tibia?

What is formed by its lower extremity?

What is the patella?

What kind of a bone is it?

What is "house-maids knee"?

How is the foot divided?

How many bones are in each division?

What is the uppermost bone of the tarsus?

With what bone does it articulate? 
What bone forms the heel proper?

How many phalanges are there in the foot?

With what do the phalanges articulate?

\section{ARTICULATIONS.}

What are articulations? How many kinds are there?

Where do we find sutural ligaments?

What is cartilage, and where is it found?

What holds the joint together?

What membrane do joints contain?

What is the character of the fluid secreted by them?

What structures enter into the formation of joints?

Why is the term synovia used?

How are joints classified according to their range of motion?

What are tendons?

What is adipose tissue? State its functions.

What condition is caused through an excess of adipose tissue? 


\section{THE MUSCULAR SYSTEM}

THE muscles constitute the fleshy parts of the body, and are the main organs of motion. They are formed of bundles of reddish fibers, endowed with the property of contractility. These bundles of fibers are enclosed in a delicate web called the perimysium, in contradistinction to the sheath of areolar tissue which invests the en-

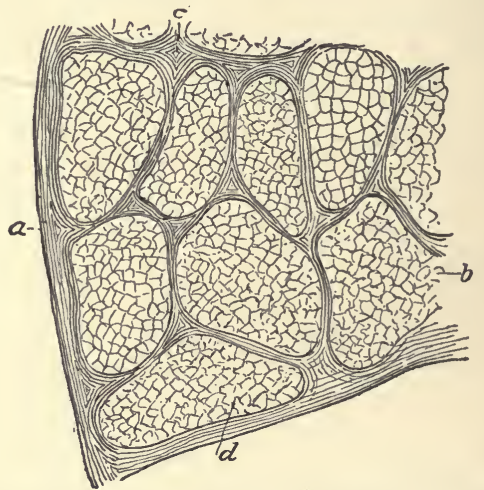

FIG. 29.-Transverse section of a muscle, magnified: $a$, Epimysium ; $b$, fasciculus; $c$, perimysium; $d$, fiber.

tire muscle, the epimysium. The bundles are termed "fasciculi"; they are prismatic in shape (see Fig. 29), of different sizes in different muscles, and, for the most part, placed parallel to one another, though they have a tendency to converge toward their tendinous attachment.

The fasciculi, or bundles of fibers, are in themselves 
very minute, but being arranged in compound bundles of successively larger size, we have ultimately only a single bundle in a muscle.

The essential characteristic of a muscle is its contractility-it contracts in length, dilates in breadth, its body becoming firm and rigid.

Muscles are said to originate at the point where they have the most fixed attachment. By insertion is understood the opposite end, or that termination or attachment that is more movable - that is, the end of the muscle that most moves the bone, or portions to which it is attached. The muscles are abundantly supplied with blood-vessels and nerves. They are frequently the seat of rheumatic disorders, as well as of inflammation.

The number of muscles in the human body is commonly estimated at 405 ; with the exception of 9, they all occur in pairs.

The muscles vary widely in size and in form. In the limbs they are of considerable length; this is true especially of the more superficial muscles, the deep ones generally being bread. They surround the bones, and form an important protection to the various joints. In the trunk the muscles are broad, flattened, and expanded, forming the parietes of the cavities which they enclose. For purposes of description, therefore, muscles are termed long, broad, short, etc.

Each muscle is invested externally by a thin cellular layer, forming what is called its sheath; this not only covers its outer surface, but penetrates its interior, in the spaces between the fasciculi, surrounding these, and serving as a bond of connection between them.

The muscles are connected with the bones, cartilages, ligaments, and skin either directly or through the medium of fibrous structures called tendons or aponeuroses. (See pages 46 and 47.) 
The muscles are capable of shortening when irritated mechanically or by nerve-stimulus, this property being called "muscular contractility or irritability."

When a muscle is attached to bone or cartilage, the fibers terminate in broad, blunt extremities upon the

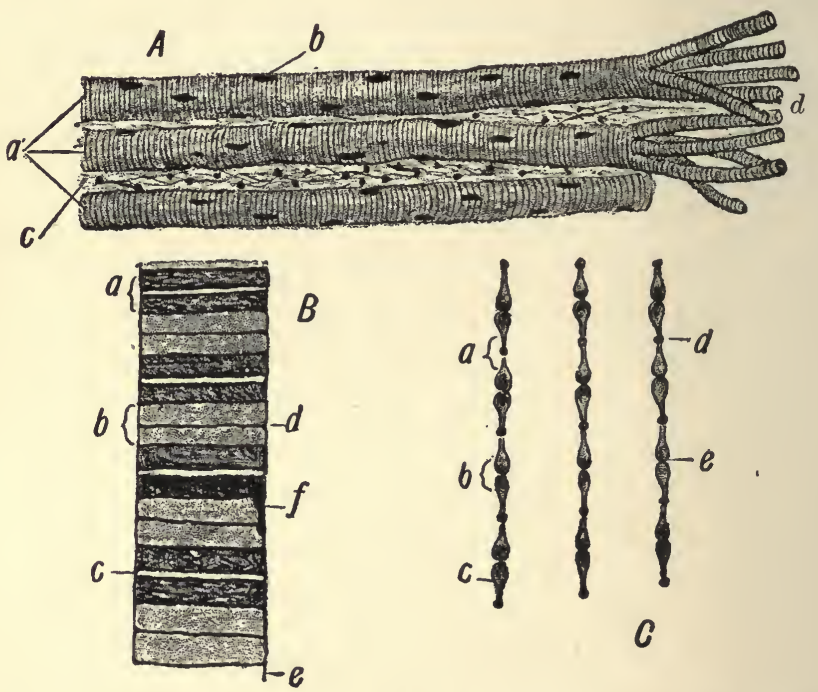

FIG. 30.-Voluntary muscle: $A$, Three voluntary fibers in long sections: $a$, Three voluntary muscle-fibers; $b$, nuclei of same; $c$, fibrous tissue between the fibers (endomysium); $d$. fibers separated into sarcostyles. $B$, Fiber (diagrammatic): $a$, Dark band; $b$, light band; $c$, median line of Hensen ; $d$, membrane of Krause ; $e$, sarcolemma ; $f$, nucleus. $C: a$, Light band; $b$, dark band; $c$, contracting elements; $d$, row of dots composing the membrane of Krause; $e$, slight narrowing of contracting element aiding in production of median line of Hensen (Leroy).

periosteum or perichondrium, and do not come into direct relation with the osseous or cartilaginous tissue. When muscles are connected with the skin, they either lie. as a flattened layer beneath it, or are connected with its areolar tissue by larger or smaller bundles of fibers, as in the muscles of the face. 
The muscles of the body are of two kinds-the volun= tary (striped muscles), which are capable of being moved or controlled by the efforts of the will, and the involun= tary (non-striped), those that are not under the control of the will. The former make up the larger bulk of the muscular system; the latter are found in the blood-vessels and in the walls of most of the hollow viscera.

For descriptive purposes the muscles of the body are arranged in divisions according to their location: as, the head, face, and neck muscles; the trunk muscles; the muscles of the upper and of the lower extremities.

As has been previously stated, in this connection only the most important points that a nurse should know, or become familiar with, will be described; as, for example, the important relation each organ and muscle bears to life, its action, and, in the case of the muscles of the head and face, the rôle they play in lending expression to the countenance.

To facilitate description, muscles are divided into groups that take their names from the regions in which they are located. Thus we have, in the head and face, the following regions: the epicranial, auricular, palpebral, orbital, superior maxillary, inferior maxillary, temporomaxillary, pterygomaxillary, etc.

\section{MUSCLES OF THE HEAD AND FACE}

The Occipital Region.-The first muscle to be described is the occipitofrontalis. You will note that the prefix occipito- relates to the occipital bone, and the suffix-frontalis, to the frontal bone, showing that the muscle extends from the posterior part of the head (occiput) to the eyebrows. All muscles derive their names in this way, and if students will study these names carefully, they will become fully acquainted with the origins and insertions of the muscles. 
The occipitofrontalis is a broad, musculofibrous layer that covers the vertex, and blends in front with the muscles of the face and laterally with the fascia of the temporal muscles. Action, raises the eyebrows and the skin of the nose and causes the transverse wrinkles of the brow; the whole scalp may be moved forward and back-

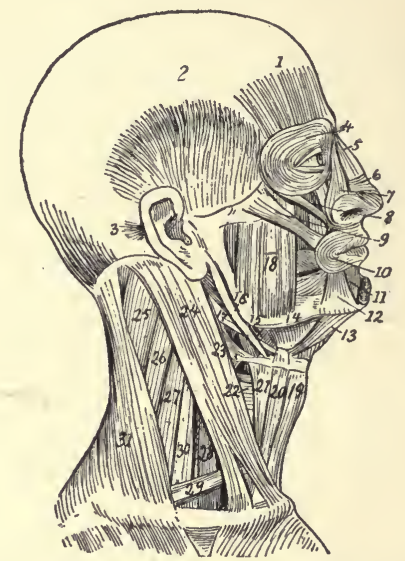

FIG. $3^{\text {I. }}$-Muscles of the right side of the head and neck: I, Frontalis; 2, temporal; 3 , retrahens aurem ; 4, orbicularis palpebrarum; 5 , pyramidalis nasi; 6 , compressor naris; 7 , levator labii superioris alæque nasi ; 8 , levator labii superioris; 9, zygomaticus major; Io, orbicularis oris; II, depressor labii inferioris; I2, depressor anguli oris; I3, anterior belly of digastric; I4, mylohyoid ; I5, hyoglossus; I6, stylohyoid; I7, posterior belly of digastric; I8, the masseter; I9, sternohyoid; 20 , anterior belly of omohyoid; 21, thyrohyoid; 22, 23, lower and middle constrictors of pharynx; 24 , sternomastoid; 25,26 , splenius ; 27 , levator scapulæ; ‘ 28 , anterior scalenus; 29 , posterior belly of omohyoid ; 30 , middle and posterior scalenus; 3I, trapezius.

ward by the action of this muscle. It is involved in expressing surprise and horror.

Blood-supply, from the occipital and posterior auricular behind, and by the supra-orbital and anterior temporai in front.

Nerve-supply, posteriorly by the posterior auricular branch of the facial, and exceptionally from the occipital 
minor nerve. Anteriorly, by the temporal branch of the temporofacial division of the facial.

The auricular region contains the three "aurem muscles," which are placed immediately beneath the skin around the external ear, and are named attollens. attrahens, and retrahens; they are fan-shaped, and their fibers converge toward the pinna or auricle.

These muscles possess very limited movement: the attollens slightly raises the ear, the attrahens draws it forward and upward, and the retrahens draws it backward.

The palpebral region contains four muscles-the orbicularis palpebrarum, corrugator supercilii, levator palpebra, and tensor tarsi.

The orbicularis palpebrarum is a sphincter muscle $v$. and surrounds the orbit and eyelids, becoming blended with the occipitofrontalis and the corrugator supercilii. Action, closes the eyelids.

The corrugator supercilii is a small, narrow, pointed muscle, situated immediately above the orbit, at the inner extremity of the eyebrow. Action, draws the eyebrows downward and inward.

The levator palpebræ will be considered when describing the muscles of the orbital region.

The tensor tarsi is a small, thin muscle. Action, draws the eyelids and the extremities of the lacrimal canal inward and compresses them against the globe of the eye; in this position they receive the tears that are secreted.

Orbital Region.-As the name implies, these muscles are connected with the orbit; there are-I levator palpebræ, 4 recti (superior, inferior, internal, and external), and 2 oblique (superior and inferior). These muscles all have their special attachments to the several parts of the eyeball, and arise from the bony structure that encloses it. Action, to control the movements of the eye. (Note the arrangement of these muscles in Fig. 32.) 
Nasal Region.--In this region there are seven pairs of muscles, which control the lip and act as levers to lift it;

- they also control the size of the anterior nares. These are, to a certain degree, muscles of expression; each muscle has its own function to perform, and each acts antagonistically to the other - that is, some dilate, while others constrict, the parts concerned in their movements.

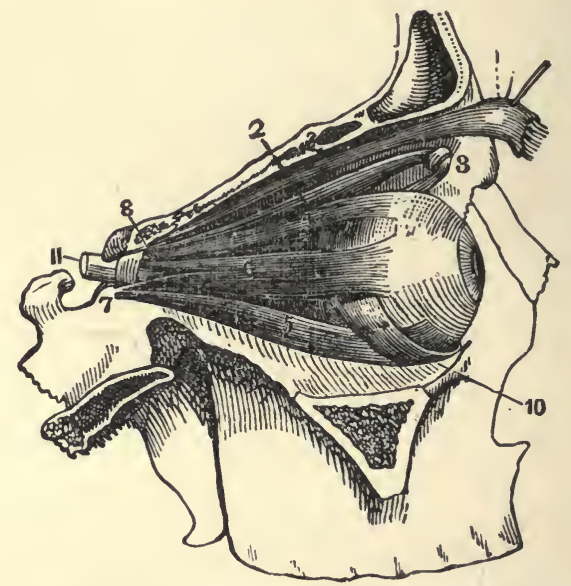

FIG. 32.-Muscles of the orbit: I, Levator palpebræ superioris; 2, obliquus superior ; 3 , pulley of obliquus superior ; 4 , rectus superior; 5 , rectus inferior; 6 , rectus externus; 7 , lower head of rectus externus; 8 , upper head of rectus externus; Io, obliquus inferior; II, optic nerve (Leidy).

Superior Maxillary Region.-In this region there are four muscles on each side; they are connected to the superior maxillary and malar bones, and arise from the lower margin of the orbit; some of them are inserted into the muscular substance of the upper lip. They are named as follows: Levator labii superioris, levator anguli oris, zygomaticus major, zygomaticus minor. (See Fig. 3I.)

Action, elevate the upper lip; draw the mouth inward and outward, as in laughing or displaying any emotion, 
as they are able to alter the shape of the mouth so that various expressions may be assumed.

Inferior Maxillary Region.-Here we have the levator labii inferioris, depressor labii inferioris, depressor anguli oris. Action of the levator labii is to raise the lower lip; it also protrudes it, and at the same time wrinkles the chin. The depressor labii inferioris draws the lip downward and outward. The depressor anguli oris draws the angle of the mouth directly backward. These muscles all blend with other muscles of the face (Fig. 3I).

Intermaxillary Region.-Contains the orbicularis oris, buccinator, and risorius. The orbicularis oris is a sphincter muscle, elliptic in form, and surrounds the oral aperture (mouth); it unites with the buccinator on each side, as well as with the several muscles of the face. Action, closes the lips.

The buccinator is a broad, thin muscle. Action, assists in the process of mastication; compresses the cheeks, so that the food is kept in immediate contact with the teeth.

The risorius has its insertion in the angle of the mouth. Action, draws out the angle of the mouth and compresses the cheek.

Temporomaxillary Region.-The two muscles in this region are of great importance; they are named the masseter and the temporal. The masseter is a short, thick muscle, consisting of two portions-superficial and deep. It arises from the malar process of the superior maxilla and zygomatic arch, and is inserted into the angle of the lower jaw. Action, a muscle of mastication.

The temporal is a broad muscle, situated on the side of the head, arising from temporal fossa and fascia, and inserted in the coronoid process of the inferior maxillary bone. Action, assists in the process of mastication. 
$\checkmark$ Pterygomaxillary Region.-In this region there are two muscles on each side - the internal and the external. They are very powerful in their action.

- The internal pterygoid is a thick, quadrilateral muscle, resembling the masseter in form and structure, and the fibers run in about the same direction.

The external pterygoid is a short, thick muscle, broader at its base, and arises by two heads-from the sphenoid bone by one head, and from the pterygoid plate of the ethmoid, palate, and maxillary bones by the other. Insertion, to the lower jaw. Action, raises the lower jaw with great force, drawing the lower jaw forward upon the upper jaw, while the masseter draws it backward, assisted by the temporal; by alternation of the muscles of each side the food is comminuted and prepared for swallowing.

\section{MUSCLES OF THE NECK}

The muscles of the neck are: Platysma myoides, sternocleidomastoid, sternohyoid, sternothyroid, thyrohyoid, omohyoid, digastric, stylohyoid, geniohyoid, geniohyoglossus, hyoglossus, styloglossus, and lingualis.

Platysma Myoides. - Arises from the deep fascia over the pectoral, deltoid, and trapezius muscles. Insertion, in the lower jaw at the angle of the mouth, and blends with the muscles of the face. Action, depresses the jaw and angle of the mouth, also wrinkles the skin of the neck; its nerve-supply is obtained from the facial nerve, through the inframaxillary branches.

$\checkmark$ Sternocleidomastoid.-Arises from the upper part of the sternum and inner border of the clavicle. Inserted in the mastoid process of the temporal bone and twothirds of curved line of the occipital bone. Action, flexes the head on the neck and chest and rotates the chin to the opposite side. Nerve-supply, from the spinal accessory, second and third cervical, anterior branches. When 
this muscle is permanently shortened upon one side, the resulting condition is known as torticollis, or "wry-neck."

Sternohyoid.-Arises from the sternum and calvicle. Inserts into the hyoid bone. Action, depresses the hyoid bone.

Sternothyroid.-Originates in the posterior part of the sternum and cartilage of the first rib. Inserts into the thyroid cartilage. Action, depresses the larynx.

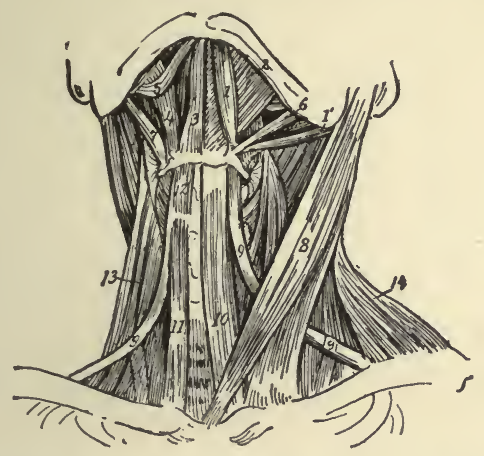

Fig. 33.-Muscles of the neck from the front: On the left side the platysma has been removed, and on the right the digastric, the stylohyoid, mylohyoid, sternohyoid, and omohyoid : I, Posterior, and $I^{\prime}$, anterior belly of digastric ; 2, mylohyoid; 3 , geniohyoid; 4 , hyoglossus ; 5 , styloglossus ; 6 , stylohyoid; 7 , stylopharyngeus; 8 , sternomastoid; 9 , 9', omohyoid; Io, sternohyoid; II, I2, sternothyroid; I3, levator scapulæ.

Thyrohyoid.-Origin from the thyroid cartilage. Insertion into the hyoid bone. Action, elevates the larynx or depresses the hyoid bone.

The omohyoid arises from the scapula, and is inserted into the hyoid bone and cartilage of the first rib. Action, depresses the hyoid bone and larynx and draws them backward and to one side.

Digastric.-Arises from two heads, with an intermediate tendon; is attached to mastoid process of the temporal bone and lower jaw. Insertion, into the hyoid bone. Action, raises the hyoid bone and depresses the 
lower jaw. Nerve-supply from the facial and inferior dental.

Stylohyoid.-Arises from the styloid process of the temporal bone. Insertion, into the body of the hyoid bone Action, same as the former.

Geniohyoid.-Arises from the inferior tubercle of the lower jaw and is inserted in the hyoid bone. Action, same as the digastric.

Geniohyoglossus.-Originates from the superior tubercle of the lower jaw. Inserted into the body of the hyoid bone, side of the pharynx, and tongue from the base to the tip. Action, protrudes as well as retracts the tongue; it also produces a longitudinal groove in this organ, as in the act of sucking. Nerve-supply, the hypoglossal.

Hyoglossus.-Arises from the hyoid bone; inserted in the sides of the tongue. Action, makes this organ convex from side to side. Nerve-supply from the hypoglossal.

Styloglossus.-Arises from the styloid process of the temporal bone. Insertion, into the side of the tongue. Action, draws the tongue upward and backward. Nerve, hypoglossal.

Lingualis.-Forms the bulk of the tongue, and is blended with the other muscles which are attached to this organ. Its action is in unison with that of the other muscles.

Palate Region.-This region is supplied with the following muscles: Levator palati, tensor palati, azygos uvulæ, palatoglossus, palatopharyngeus. They arise from adjacent bony structures and have control over the soft palate and uvula, besides assisting in the acts of speech, deglutition, and mastication.

Pharyngeal Region.-The muscles found in the pharyngeal region are the following: Three constrictorsthe inferior, the superior, and the medius; the stylopharyngeus and the palatopharyngeus. 
The inferior constrictor arises from the cricoid and thyroid cartilages, and is inserted into its fellow of the opposite side. Action, constricts the pharynx during swallowing.

The middle constrictor has its origin in the hyoid bone and stylohyoid ligament. Insertion, into its fellow of the opposite side. Action, same as inferior constrictor.

The superior constrictor arises from the pterygoid plate and palate bone, as well as from the hyoid. Insertion, into the occipital bone. Action, same as inferior constrictor.

The stylopharyngeus arises from the inner side of the base of the styloid process of the temporal bone; it is inserted into the thyroid cartilage and blends with the constrictor muscles. Action, elevates the pharynx.

The palatopharyngeus has its origin in the soft palate. Insertion, into the thyroid cartilage and side of the pharynx. Action, closes the posterior fauces.

All the muscles just described, except the palatopharyngeus, are controlled by the spinal accessory, glossopharyngeal, pharyngeal plexus, external and recurrent laryngeal nerves; the palatopharyngeus is controlled by the palatoglossus nerve.

The Pharynx.-The pharynx is a musculomembranous sac, about 5 inches long, extending from under the base of the skull to a point corresponding to the sixth cervical vertebra. It is widest above and narrowest below, where it terminates in the esophagus. It is attached to the styloid processes of the temporal bone, and presents seven openings: the two posterior nares, two Eustachian tubes, the mouth, the larynx, and the esophagus. For purposes of description it is divided into three parts-nasal, oral, and laryngeal. It is attached to two cartilages-the cricoid and the thyroid - and has three coats - an internal mucous membrane, a middle fibrous layer, and an external muscular coat. 
Anterior Vertebral Region.--This region contains four muscles-the rectus capitis anticus major, the rectus capitis anticus minor, the rectus lateralis, the longus colli. The lateral vertebral region contains three muscles-the scalenus anticus, medius, and posticus. Action of the several muscles, to control the movements of the head and neck; by means of their several attachments the head may be moved in any direction.

\section{MUSCLES OF THE TRUNK}

These are divided into groups, according to the region in which they are located: thus we have the muscles of the back, of the abdomen, of the thorax, and of the perineum.

The muscles of the back are divided into five layers, of which the more superficial layer is of most interest to the nurse. (In this connection the illustrations should be carefully studied, in order that an accurate knowledge of the construction of these muscles may be obtained.)

The first of these to be described is the large muscle situated at the upper and back part of the neck and shoulders, this is known as the trapezius.

$\checkmark$ Trapezius.-Origin, from the occipital bone and the processes of the seventh cervical and the twelve dorsal vertebræ, as well as from the ligamentum nuchæ. Insertion, into the clavicle and scapula. Action, draws the shoulder upward, backward, and outward; it also moves the scapula on the chest.

$\checkmark$ Latissimus Dorsi.-Origin, from the six lower dorsal vertebræ and the crest of the ilium; being a broad muscle, it covers the lumbar and lower half of the sacral region, and is one of the most important muscles of the body. Insertion, into the humerus. Action, draws the humerus downward and backward while rotating it inward; it raises the lower ribs in forcible inspiration; it 
also assists the other muscles of the chest, as in climbing, pulling, etc.

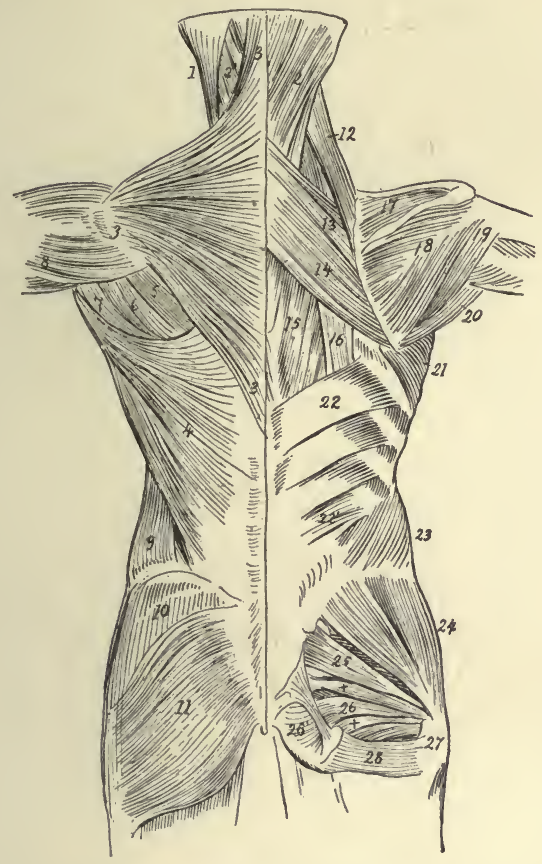

FIG. 34.-Muscles of the trunk from behind (left side, superficial; right side, deep): I, Sternomastoid; 2 , splenius; 3 , trapezius; 4, latissimus dorsi ; 5, infraspinatus; 6 , teres minor; 7 , teres major; 8 , deltoid ; 9 , external oblique of abdomen; Io, gluteus medius; II, gluteus maximus; I2, levator anguli scapulæ; $1_{3}$, rhomboideus minor; I4, rhomboideus major; 15. part of longissimus dorsi ; I6, tendons of insertion of iliocostalis ; I7, supraspinatus; 18 , infraspinatus; I9, teres minor; 20, teres major; 21 , serratus magnus; 22 , upper, and $22^{\prime}$, lower part of serratus posticus inferior ; 23 , internal oblique; 24 , gluteus medius; 25 , pyriformis and superior and inferior gemelli; $26,26^{\prime}$, portions of obturator internus; 27 , tendon of obturator internus; 28 , quadratus femoris.

('The student should refer to the illustrations, noting the relation that the several muscles bear to the act of respiration, as well as the number that are concerned in the act of moving the trunk in various positions.) 
r The muscles of the abdomen are: The obliquus externus; obliquus internus; transversalis; rectus; pyramidalis, and quadratus lumborum.

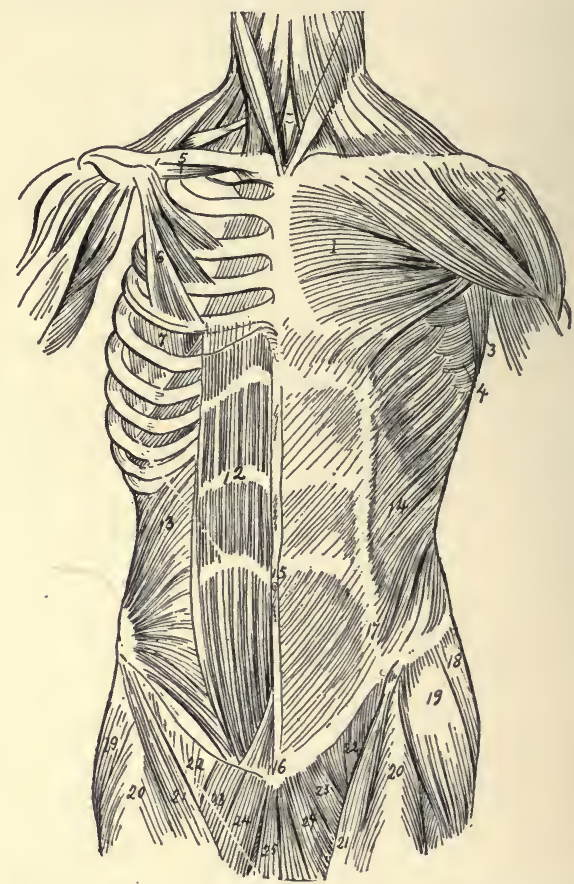

FIG. 35.-Muscles of the trunk from in front (left side, superficial; and right side, deep): I, Pectoralis major; 2, deltoid; 3 , portion of latissimus dorsi ; 4, serratus magnus; 5 , subclavius; 6 , the pectoralis, sternocostal portion; 7 , serratus magnus; 12, rectus abdominis ; 13 , internal oblique ; I4, external oblique; 15 , abdominal aponeurosis and tendinous intersections of rectus abdominis; 16 , over symphysis pubis; 17 , linea semilunaris; 18 , gluteus medius; I9, tensor vaginæ femoris; 20 , rectus femoris; $2 \mathrm{I}$, sartorius; 22, femoral part of iliopsoas; 23 , pectineus; 24 , adductor longus ; 25, gracilis.

- The external oblique arises from the eight inferior ribs; it is inserted into the anterior half of the iliac crest, and, mingling with its fellow of the opposite side, it forms the linea alba. It is connected above with the ensiform carti- 
lage, and below with the pubis. (That portion of the aponeurosis of the external oblique which extends from the anterior superior spine of the ilium to the spine of the pubis is known as Poupart's ligament.) Gimbernat's ligament is an extension of the latter to the iliopectineal line. Action, compresses the viscera, flexes the thorax on the pelvis or the pelvis on the thorax, according to which is the fixed point, and also assists expiration. Nervesupply, the lower intercostals.

The internal oblique arises from Poupart's ligament, from the middle lip of the crest of the ilium, and from the lumbar fascia. Insertion, crest of os pubis with the transversalis muscle-forming the conjoined tendon, which strengthens the external abdominal ring; it also blends with the external oblique to form the linea alba, and with the transversalis muscles. Action, same as that of the external oblique. Nerve-supply, lower intercostals, iliohypogastric, and ilio-inguinal.

The muscles just described are broad and cover the greater part of the abdominal wall; they are, therefore, to be considered in operations in this locality.

The muscles of the abdomen that have not been described may be studied from the illustrations. The student should observe especially the linea alba, a tendinous raphé or cord seen as a dark, perpendicular streak in the middle of the abdomen. This is of some importance, and is of interest to the nurse, especially in regard to the discoloration that takes place in pregnant women.

Gray says: The abdominal muscles have a threefold action: They compress the abdominal wall during the process of defecation; they assist in the act of respiration, and they help in the expulsion of the fetus; the urine is expelled through the agency of this combination of muscular force, and by it, through upward pressure, the stomach is relieved of its contents. From this it will be seen 
that the action of these muscles should be studied closely, making frequent reference to the illustrations.

Muscles of the Thorax.-The muscles of the thorax $\checkmark$ are the external and internal intercostals, the infracostales, the triangularis sterni, and the levator costarum; of these, the most important are the intercostals. They are all connected with the bony structure of the thorax, and are all concerned in respiration.

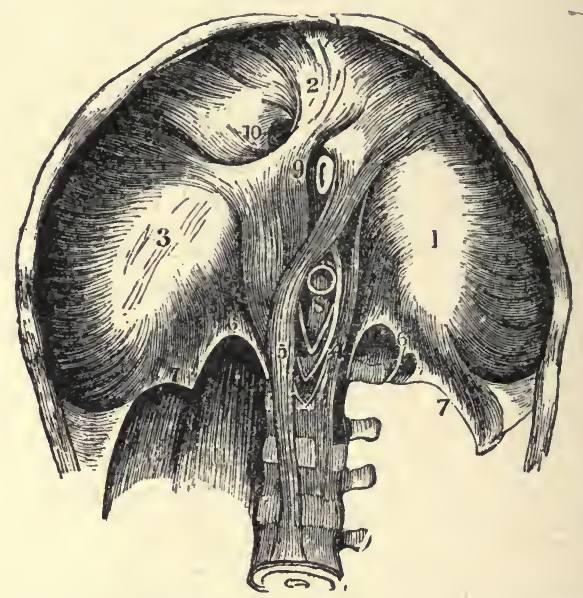

FIG. 36.-The diaphragm, inferior surface: $1,2,3$, Central cordiform tendon; 4,5 , crura; 6 , internal arcuate ligament; 7 , external arcuate ligament; 8, aortic opening; 9, esophageal; Io, opening for vena cava (Leidy).

- Diaphragmatic Region.-The diaphragm is a thin, musculofibrous septum, separating the thorax from the abdominal cavity. It is divided into two parts-a greater and a lesser portion. It is convex toward the chest; its circumference is connected with the ribs and the vertebræ. It contains openings for the transmission of the aorta, esophagus, vena cava, nerves, arteries, and veins. Action, a muscle of respiration. 


\section{MUSCLES OF THE UPPER EXTREMITY}

\section{MUSCLES OF THE SHOULDER AND ARM}

\author{
Pectoralis major. \\ Pectoralis minor. \\ Subclavius. \\ Serratus magnuș. \\ Deltoid. \\ Subscapularis. \\ Supraspinatus.
}

\author{
Teres major. \\ Teres minor. \\ Coracobrachialis. \\ Biceps flexor cubiti. \\ Brachialis anticus. \\ Triceps extensor cubiti. \\ Subanconeus.
}

The pectoralis major muscle is located upon the chest. Origin, from the clavicle, sternum, and upper six costal cartilages. Insertion, into the humerus. Action, draws arm downward and forward.

Pectoralis Minor.-Origin, from the third, fourth, and fifth ribs. Insertion, into the coracoid process of the scapula. Action, depresses the shoulder and assists in the elevation of the ribs during the act of inspiration.

The subclavius arises from the first costal cartilage and rib, and is inserted into the inner surface of the clavicle. Action, draws the clavicle downward.

The serratus magnus arises by nine fleshy digitations from the outer surface of the eight upper ribs; it is inserted into the posterior border of the scapula, Action, elevates ribs in inspiration; fixes scapula in act of pushing.

The deltoid is a thick, triangular muscle forming the convexity of the shoulder. Origin, from the upper border of the clavicle, outer and upper surfaces of the acromion process, and lower border of the scapula. Insertion, into the outer side of the humerus. Action, raises the arm to a right angle, and draws it forward or backward.

The subscapularis arises from the inner two-thirds of the subscapular fossa; is inserted into lesser tuberosity of the humerus. Action, rotates the humerus inward as well as adducts the same. Nerve, subscapular. 
The supraspinatus arises from the supraspinous fossa. Inserted into the upper facet on the greater tuberosity of the humerus. Action, assists the deltoid; fixes the head of the humerus in the socket. Nerve, suprascapular.

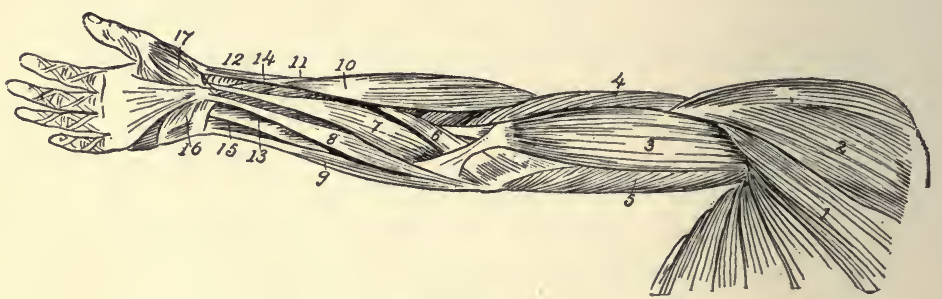

FIG. 37.

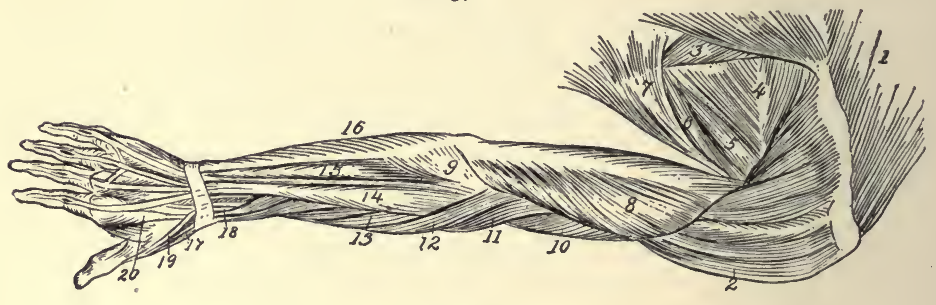

FIG. 38.

FIG. 37.-Superficial muscles of shoulder and arm (from in front): I, Pectoralis major; 2 , deltoid; 3 , biceps; 4 , brachialis anticus; 5 , triceps; 6 , pronator radii teres; 7 , flexor carpi radialis; 8 , palmaris longus; 9 , flexor carpi ulnaris Io, supinator longus ; II, extensor ossis metacarpi pollicis ; 12, extensor brevis pollicis; I3, flexor sublimis digitorum; I4, flexor longus pollicis; flexor profundus digitorum; I6, palmaris brevis; I7, abductor pollicis.

FIG. 38.-Superficial muscles of shoulder and arm (from behind): I, Trapezius; 2 , deltoid; 3 , rhomboideus major; 4 , infraspinatus; 5 , teres minor; 6, teres major; 7, latissimus dorsi ; 8, triceps; 9, anconeus; 10, brachialis anticus; II, supinator longus; I2, extensor carpi radialis longior; I3, extensor carpi radialis brevior; I4, extensor communis digitorum; I5, extensor carpi ulnaris ; I6, flexor carpi ulnaris ; I7, extensor ossis metacarpi pollicis ; I8, extensor brevis pollicis; I9, tendon of extensor longus pollicis.

Infraspinatus.-Origin, infraspinous fossa. Insertion, into the middle facet of the greater tuberosity_of the humerus. Action, rotates the humerus outward. Nerve, suprascapular. 
Teres Minor.-Origin, upper two-thirds of the axillary border of the scapula. Insertion, into the lower facet of the greater tuberosity of the humerus. Action, rotates the humerus outward. Nerve, circumflex.

Teres Major.-Origin, lower border of infraspinous fossa, and is inserted into the bicipital groove of the humerus. Action, draws the humerus downward and backward, when raised also rotates it inward. Nerve, lower subscapular.

Coracobrachialis.-Origin, from the coracoid process of the scapula. Insertion, middle of inner side of the humerus. Action, draws the humerus forward and inward, as well as elevates same. Nerve, musculocutaneous.

Biceps Flexor Cubiti.-Origin, by two heads: Short head, from the coracoid process of the scapula; long head, from upper margin of the glenoid cavity by a round tendon continuous with the glenoid ligament. Insertion, by a tendon into the back part of the tuberosity of the radius and by an aponeurosis into the fascia of the forearm, which renders this fascia tense. Nerve, musculocutaneous.

Brachialis Anticus.-Origin, anterior surface of the humerus, embracing the insertion of the deltoid. Insertion, into the inferior surface of the coronoid process of the ulna. Action, flexes the forearm. Nerve, musculocutaneous and musculospiral.

Triceps Extensor Cubiti.-Origin, arises by three heads, $大$ the long, external, and internal; long head, from the glenoid fossa, the external, from the posterior surface of the shaft of the humerus between the upper part of the musculospiral groove and the insertion of the teres minor; internal, from the posterior surface below the musculospiral groove. Insertion, into the olecranon process of the ulna. Action, extends the forearm. Nerve, musculospiral.

Subanconeus.- Origin, above the olecranon fossa of the humerus. Insertion, into the posterior ligament of the 
elbow. Action, prevents the capsule from injury during extension of the arm. Nerve, musculospiral.

\section{MUSCLES OF THE FOREARM}

$\begin{array}{ll}\text { Superficial layer: } & \left\{\begin{array}{l}\text { Pronator teres. } \\ \text { Flexor carpi radialis. } \\ \text { Palmaris longus. } \\ \text { Flexor carpi ulnaris. } \\ \text { Flexor sublimis digitorum. }\end{array}\right. \\ \text { Deep layer: } & \left\{\begin{array}{l}\text { Flexor profundus digitorum. } \\ \text { Flexor longus pollicis. } \\ \text { Pronator quadratus. }\end{array}\right. \\ \text { Radial region: } & \left\{\begin{array}{l}\text { Supinator longus. } \\ \text { Extensor carpi radialis longior. } \\ \text { Extensor carpi radialis brevior. }\end{array}\right. \\ \text { Posterior radio-ulnar region } & \left\{\begin{array}{l}\text { Extensor communis digitorum. } \\ \text { Extensor minimi digiti. } \\ \text { Extensor carpi ulnaris. } \\ \text { Anconeus. }\end{array}\right. \\ \text { Deep layer: } & \left\{\begin{array}{l}\text { Supinator brevis. } \\ \text { Extensor ossis metacarpi pollicis. } \\ \text { Extensor brevis pollicis. } \\ \text { Extensor longus pollicis. } \\ \text { Extensor indicis. }\end{array}\right.\end{array}$

The pronator teres arises from the internal condyle of the humerus and from the coronoid process of the ulna. Inserted, outer side of shaft of radius. Action, pronates forearm. Nerve, median.

Flexor Carpi Radialis.-Origin, internal condyle of humerus. Insertion, metacarpal bone of index-finger. Action, flexes wrist. Nerve, median.

Palmaris Longus.-Origin, from internal condyle. Insertion, in the annular ligament and palmar fascia. Action, makes the palmar fascia tense. Nerve, median.

The flexor carpi ulnaris arises by two heads: First, from the internal condyle; second, from the olecranon 
process of the ulna. Insertion, fifth metacarpal bone, annular ligament, and pisiform bone. Action, flexes the wrist. Nerve, ulnar. (See Figs. 37 and 38.)

Flexor Sublimis Digitorum.-Origin, by three heads: First, from the inner condyle; second, coronoid process of ulna; third, oblique line of radius. Insertion, into the second phalanges by four tendons. Action, flexes second phalanges. Nerve, median.

The flexor profundus digitorum arises from shaft of ulna. Insertion, into last phalanges by four tendons. Action, flexes the phalanges. Nerve, median.

Flexor Longus Pollicis.-Origin, shaft of radius and coronoid process of ulna. Insertion, last phalanx of thumb. Action, flexes the phalanx. Nerve, anterior interosseous.

Pronator Quadratus.-Origin, from lower fourth of ulna. Inserted, in lower one-fourth of radius. Action, pronates the hand. Nerve, anterior interosseous.

Supinator Longus.- Origin, upper two-thirds of external condyloid ridge of humerus. Insertion, styloid process of radius. Action, flexes forearm. Nerve, musculospiral.

Extensor Carpi Radialis Longior.-Origin, lower onethird of external condyloid ridge of humerus. Insertion, base of second metacarpal bone. Nerve, musculospiral. Action, extends wrist.

Extensor Carpi Radialis Brevior--Origin, external condyle of humerus. Insertion, base of second and third metacarpal bones. Action, extends wrist. Nerve, posterior interosseous.

Extensor Communis Digitorum.-Origin, external condyle of humerus. Insertion, the second and third phalanges. Action, extends the fingers. Nerve, posterior interosseous.

Extensor Minimi Digiti.-Origin, external condyle of humerus. Insertion, in the second and third phalanges of 
little finger. Action, extensor of little finger. Nerve, posterior interosseous.

Extensor Carpi Ulnaris.-Origin, by two heads: First head, external condyle of humerus; second head, posterior border of ulna. Insertion, base of fifth metacarpal bone. Action, extends wrist. Nerve, posterior interosseous.

Anconeus.-Origin, back and external condyle of humerus. Insertion, olecranon process and shaft of ulna. Action, extends forearm. Nerve, musculospiral

Supinator Brevis.-Origin, external condyle o nerus and oblique line of ulna. Insertion, neck of rad ind its bicipital tuberosity. Action, supinates the forearm. Nerve, posterior interosseous.

Extensor Ossis Metacarpi Pollicis.-Origin, back of radius and ulna and interosseous membrane. Insertion, base of metacarpal bone of thumb and fascia. Action, extends the thumb. Nerve, posterior interosseous.

Extensor Brevis Pollicis.-Origin, back of radius. Insertion, inner part of the base of first phalanx of thumb. Action, extends the first phalanx of thumb and abducts the wrist. Nerve, posterior interosseous.

Extensor Longus Pollicis.-Origin, posterior part of the ulna and interosseous membrane. Action, extends second phalanx of the thumb and abducts the wrist. Nerve, posterior interosseous.

Extensor Indicis. - Origin, posterior part of ulna. Insertion, second and third phalanges of index-finger. Action, extends the index-finger. Nerve, posterior interosseous.

\section{MUSCLES OF THE HAND}

Abductor pollicis.

Opponens pollicis.

Flexor brevis pollicis.

Abductor obliquus pollicis.

Adductor transversus pollicis.

Palmaris brevis.
Abductor minimi digiti.

Flexor brevis minimi digiti.

Opponens minimi digiti.

Lumbricales.

Dorsal interossei (4).

Palmar interossei (3). 
Abductor Pollicis.-Origin, from trapezium, scaphoid, annular ligament, and palmar fascia. Insertion, first phalanx of thumb. Action, abducts and flexes first phalanx of thumb. Nerve, median.

Opponens Pollicis.-Origin, trapezium and anterior ligament. Insertion, metacarpal bone of thumb. Action, flexes and opposes thumb. Nerve, median, palmar division.

Flexor Brevis Pollicis.-Origin, by two heads: Outer, lower border of superior annular ligament and ridge of trapezium; inner, os magnum, and base of first, second, and third metacarpal bones. Insertion, base of first phalanx of thumb. Action, flexes the thumb at metacarpal articulation. Nerve, outer head, median; inner head, deep ulnar.

Adductor Obliquus Pollicis.-Origin, from os magnum, second and third metacarpal bones. Insertion, base of first phalanx of thumb. Action, draws the thumb inward toward the middle line. Nerve, ulnar.

Adductor Transversus Pollicis.-Origin, third metacarpal bone. Insertion, base first phalanx of thumb. Action, flexes the phalanx, adducts the thumb. Nerve, ulnar.

Palmaris Brevis.-Origin, from the annular ligament and palmar fascia. Insertion, skin on inner border of palm. Action, wrinkles the skin on the palmar side. Nerve, ulnar.

- Abductor Minimi Digiti.-Origin, pisiform bone. Insertion, base of first phalanx of little finger. Action, draws the little finger from the middle line and flexes the metacarpophalangeal joint. Nerve, ulnar.

Flexor Brevis Minimi Digiti.-Origin, from the unciform bone and annular ligament. Insertion, first phalanx of little finger. Action, flexes the first phalanx. Nerve, ulnar. 
Opponens Minimi Digiti.-Origin, from the unciform bone. Insertion, whole length of the fifth metacarpal bone. Action, flexes the fifth metacarpal, draws it forward, and makes the hollow of the palm of hand. Nerve, ulnar.

Lumbricales.-Origin, tendon of the deep flexors. Insertion, in the dorsum of each finger. Action, flex the first and extend the last two phalanges. Nerve, two outer by the median, two inner by the ulnar.

Dorsal Interossei.-Origin, four in number by two heads, from the sides of the metacarpal bones. Insertion, into base of first phalanges of fingers. Action, flex the first and extend the last two phalanges, abduct the fingers. Nerve, ulnar.

Palmar Interossei (three in number).-Origin, by single heads, from the palmar side of the second, fourth, and fifth metacarpals. Insertion, into the second, fourth, and fifth phalanges. Action, flex the first, extend the last two phalanges, adduct the fingers toward the middle line. Nerve, ulnar.

\section{MUSCLES OF THE LOWER EXTREMITY}

For convenience in description the muscles of the leg will be divided into groups according to their various locations, only the most important ones with which the nurse should become acquainted being described.

The muscles of the iliac or inguinal region are:

Psoas magnus.

Psoas parvus.

Iliacus.

The psoas magnus is a long, fusiform muscle, situated on the side of the lumbar region of the spine and margin of the pelvis. Origin, from the transverse processes of the last thoracic and of all the lumbar vertebræ. Insertion, into the lesser trochanter of the femur. Action, flexes the thigh on the pelvis, or vice versa; also rotates the femur 
outward. Nerve-supply, anterior branches of the second and third lumbar.

The psoas parvus is a long, slender muscle, situated in front of the psoas magnus. It arises from the last thoracic and the first lumbar vertebræ. Insertion, iliac fascia and iliopectineal eminence. Action, makes the iliac fascia tense. Nerve, anterior branch of first lumbar.

* The iliacus is a flat, radiated muscle that fills up the whole of the iliac fossa (internal), from which point, as well as from the inner margin of the iliac crest and sacrum, it originates. Inserted, with the tendon of the psoas magnus, into the lesser trochanter of the femur. Action, same as the psoas magnus. Nerve, anterior crural.

The muscles of the anterior femoral region are:

Tensor vaginæ femoris.

Sartorius.

Rectius femoris.

Vastus externus.

Vastus internus.

Crureus.

Subcrureus.

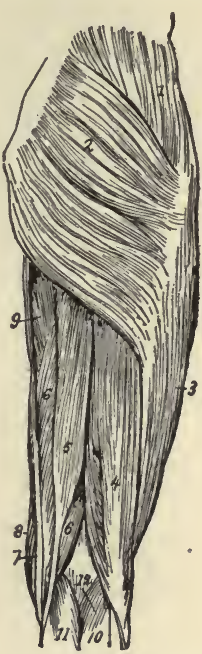

FIG. 39.-Superficial muscles of hip and thigh (from behind): I, Gluteus medius ; 2 , gluteus maximus; 3 , vastus externus ; 4 , biceps flexor cruris ; 5 , semitendinosus; 6 , semimembranosus; 7 , gracilis ; 8 , sartorius ; 9 , adductor magnus; Io, II, gastrocnemius; 12 , origin of plantaris.

The tensor vaginæ femoris is a short, flat muscle, situated at the upper and outer side of the thigh. Origin, outer part of the crest of the ilium and anterior superior spinous process. Inserted in the fascia lata at the outer side of the thigh. Action, internal rotator of the thigh. Nerve, superior gluteal. 
The sartorius, the longest muscle in the body, is a flat, narrow, ribbon-like muscle that arises from the anterior superior spinous process of the ilium, passing down the

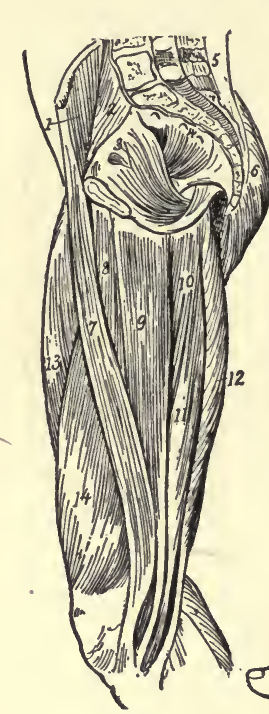

FIG. 40.

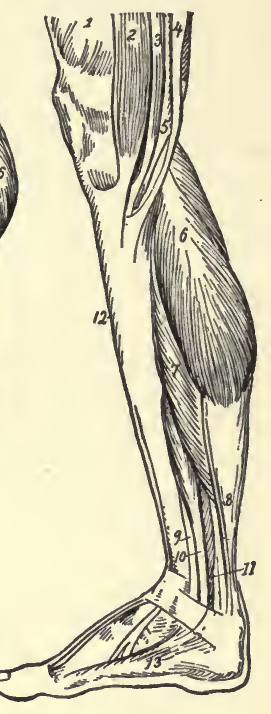

FIG. 4I.

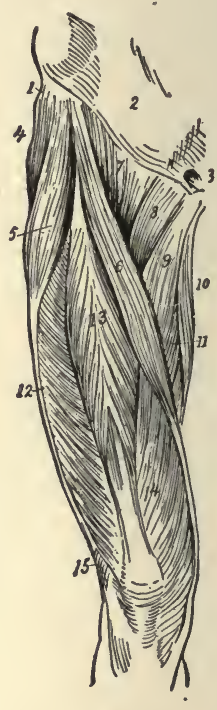

FIG. 42.

FIG. 40.-Muscles of the inner side of thigh and interior of pelvis: $\mathbf{I}$, Iliacus; 2, psoas magnus; 3 , obturator internus; 4 , pyriformis; 5 , erector spinæ; 6 , gluteus maximus; 7 , sartorius; 8 , adductor longus; 9 , gracilis ; IO, adductor magnus ; II, semimembranosus; I2, semitendinosus; 13 , rectus femoris; 14 , vastus internus.

FIG. 4I.-Superficial muscles of the leg from inner side: I, Vastus internus; 2 , sartorius; 3 , gracilis ; 4 , semitendinosus ; 5 , semimembranosus ; 6 , inner head of gastrocnemius; 7 , soleus; 8 , tendon of plantaris; 9 , tendon of tibialis posticus; Io, flexor longus digitorum; II, flexor longus hallucis; I2, tibialis anticus; I3, abductor hallucis.

FIG. 42.- Superficial muscles of front of thigh: I, Insertion of external oblique into iliac crest; 2 , aponeurosis of external oblique ; 3 , external abdominal ring; 4 , gluteus medius; 5 , tensor vaginæ femoris; 6 , sartorius; 7, iliopsoas; 8, pectineus; 9 , adductor longus; Io, gracilis ; II, adductor magnus; 12, vastus externus; 13 , rectus femoris; 14 , vastus internus; 15 , biceps flexor cruris.

inner side of the thigh vertically to the inner side of the knee. Insertion, into the inner surface of the shaft of the tibia. Action, flexes the leg upon the thigh, as well as 
the thigh upon the pelvis. Also called the tailors' muscle. Nerve, anterior crural.

\& The rectus femoris arises from the anterior inferior iliac spine. Insertion, in the patella. Action, flexes the hipjoint. Nerve, anterior crural.

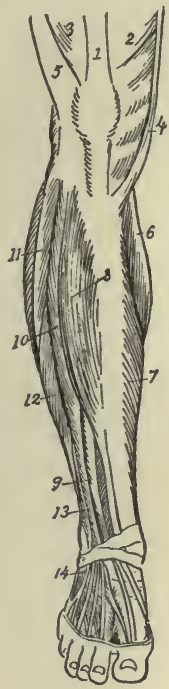

FIG. 43.

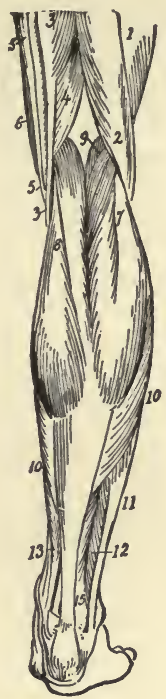

FIG. 44.

FIG. 43.-Muscles of leg and foot (from before): I, Tendon of rectus femoris ; 2 , vastus internus ; 3 , vastus externus ; 4 , sartorius ; 5 , iliotibial band; 6 , inner head of gastrocnemius; 7 , inner part of soleus; 8 , tibialis anticus; 9 , extensor proprius hallucis; ro, extensor longus digitorum; II, peroneus longus; $I_{2}$, peroneus brevis; $I_{3}$, peroneus tertius; $I_{4}$, origin of extensor brevis digitorum.

FiG. 44.--Superficial muscles of leg (from behind) : r, Vastus externus; 2 , biceps flexor cruris ; 3 , semitendinosus ; 4 , semimembranosus; 5 , gracilis ; 6 , sartorius; 7 , outer, and 8 , inner, head of gastrocnemius; 9 , plantaris; ro, soleus; II, peroneus longus; $I_{2}$, peroneus brevis; $I_{3}$, flexor longus digitorum; $I_{4}$, tibialis posticus; ${ }_{15}$, lower fibers of flexor longus hallucis.

Vastus Externus.-Origin, tubercle of the femur and the great trochanter. Inserted into the outer side of the patella. Action, extends the leg. Nerve, anterior crural. 
Vastus Internus and Crureus.-Origin, from the inner side of the femur. Insertion, into the patella. Action, extends the leg. Nerve, anterior crural.

Subcrureus.-Arises by two heads from the femur. Inserted in the upper portion of the synovial pouch of the knee-joint. Action, dráws the synovial sac upward. Nerve, anterior crural.

The muscles of the internal femoral region are:

Gracilis.

Pectineus.
Adductor longus.

Adductor magnus.

Adductor brevis.

Gracilis.-Origin, ramus of the os pubis. Inserted into the inner side of the tibia. Action, flexes the leg, rotates it inward, and adducts the thigh. Nerve, the obturator.

Pectineus.-Origin, from the iliopectineal line. Inserted into the lesser trochanter. Action, flexes and adducts the thigh. Nerves, anterior crural and obturator.

The adductor longus magnus and brevis arise from the os pubis, and are inserted into the inner side of the femur. Action, adduct, rotate, and flex the thigh. Nerve, obturator and sciatic.

The gluteal region contains the largest muscles of the body; these are as follows:

Gluteus maximus.

Gluteus medius.

Gluteus minimus.

Pyriformis.
Obturator internus.

Gemellus superior.

Gemellus inferior.

Obturator externus.

Quadratus femoris.

$\chi$ The gluteus maximus is the most superficial muscle in this region. It is a broad, thick, fleshy muscle, quadrilateral in shape, and forms the nates; it is the most important muscle in maintaining the body in an erect posture. It arises from the ilium, sacrum, and coccyx, and is inserted into the great trochanter. Action, holds the 
trunk erect, extends, abducts, and rotates the thigh outward. Nerve, inferior gluteal.

Gluteus Medius.-Arises from the outer surface of the ilium. It is inserted into the great trochanter. Action, supports the trunk, flexes and abducts the thigh, and rotates it inward. Nerve, superior gluteal.

Gluteus Minimus.-Arises from the outer side of the ilium, and is inserted in the great trochanter. Action, flexes, abducts, and rotates the thigh inward; it also assists in holding the trunk erect. Nerve, superior gluteal.

The pyriformis is a flat muscle, arising by three digitations from the sacrum and ilium. It is inserted into the great trochanter. Action, rotates thigh outward, abducts it as well, and draws the pelvis forward. Nerves, branches of sacral plexus.

The obturator internus is situated partly in the pelvis, and arises from the margin of the obturator foramen. It is inserted into the inner part of the great trochanter. Action, draws the pelvis forward and assists in external rotation and abduction of the thigh. Nerve, branch of the sacral plexus.

Gemellus Superior.-Arises from the ischium, and is inserted into the inner part of the great trochanter. Action, rotates the thigh outward. Nerve, branch of sacral plexus.

Gemellus Inferior.-Arises from the ischium, and is inserted into the great trochanter of the femur. Action, external rotator of thigh. Nerve, branch of sacral plexus.

The obturator externus is a flat, triangular muscle, situated on the outside of the pelvis. Arises from the margin of obturator foramen, and is inserted into the femur. Action, rotates thigh outward. Nerve-supply, from branch of obturator.

The quadratus femoris is a short, flat muscle, quadrilateral in shape. Origin, from the tuberosity of the 
ischium. Insertion, into the posterior intertrochanteric line. Action, abducts the thigh and rotates it outward. Nerve, branch of the sacral plexus.

The muscles of the posterior femoral region are the biceps, the semitendinosus, and the semimembranosus.

The biceps is a large muscle situated on the outer part of the thigh. It arises by two heads, the long from the tuberosity of the ischium and the short from the linea aspera. Insertion, head of the fibula, the tendon thereof splitting, one band embracing the lateral ligament and the other extending to the outer tuberosity of the tibia; this muscle is called the outer hamstring. Action, extends the hip, flexes the leg, after which it rotates it outward. Nerve, great sciatic.

The semitendinosus is situated on the inner side of the thigh; this is a remarkable muscle, as it is provided with a very extensive tendon. It arises from the tuberosity of the ischium and is associated with the biceps; it continues downward, the muscular belly becoming tendinous a little below the middle of the thigh. It is inserted into the upper and inner part of the tibia. Action, extends the hip, flexes the leg on the thigh, and rotates it inward. Nerve, great sciatic.

The semimembranosus arises from the tuberosity of the ischium. It is inserted into the inner and back part of the tuberosity of the tibia. Action, flexes the leg on the thigh and rotates it inward. Nerve, great sciatic.

Muscles of the Leg.-Having studied the muscles of the thigh and hip, those of the leg and foot will now be considered. Of these, only the most important, and especially the most superficial, will be described.

There are thirteen muscles in the leg; these are as follows :

The tibialis anticus, extensor longus digitorum, ex- 
tensor longus, hallucis, peroneus tertius, gastrocnemius, plantaris, popliteus, flexor longus hallucis, flexor longus digitorum, tibialis posticus, peroneus longus, peroneus brevis.

The deep fascia forms a complete investment of the entire region of the leg, and is attached to the inner side of the tibia.

The tibialis anticus is a thick, fleshy muscle, situated on the outer side of the tibia. It arises from the tuberosity and shaft of the tibia, the fibers thereof terminating in a tendon that passes under the annular ligament and is inserted into the internal cuneiform bone and base of the first metatarsal. Action, flexes the ankle-joint and raises the inner border of the foot. Nerve, anterior tibial.

The extensor longus hallucis is a thin, elongated, and flattened muscle. It arises from the anterior surface of the fibula and from the interosseous membrane; the fibers pass downward and terminate in a tendon that passes under the annular ligament, and is inserted in the last phalanx of the great toe. Action, same as the tibialis anticus. Nerve, anterior tibial.

The extensor longus digitorum is an elongated, flattened muscle, situated most externally of all the muscles in front of the leg. It arises from the tuberosity of the tibia and shaft of the fibula, extends downward, and divides into four tendons, which are inserted in second and third phalanges of the four lesser toes. Action, extends the toes, flexes the ankle, and everts the foot. Nerve, anterior tibial.

The gastrocnemius, the most important muscle of the posterior part of the leg, is an elongated, flattened muscle. It forms the calf proper. It arises from the condyles of the femur by two heads. Insertion, with the tendons of the soleus and plantaris to form the tendo Achillis, which is finally inserted into the tuberosity of the os calcis. 
Action, extends the foot and rotates it slightly inward. Nerve, internal popliteal.

The nurse should ascertain, from the illustrations, the location of the other muscles of the leg, particularly of the flexors, which assist in the several movements of the leg and foot.

The Muscles of the Foot.-The muscles of the foot are the following: The extensor brevis digitorum, dorsal interossei, abductor hallucis, abductor minimi digiti, flexor brevis digitorum, flexor accessorius, lumbricales, flexor brevis hallucis, adductor transversus hallucis, flexor brevis minimi digiti, transversus pedis, and plantar interossei.

While an accurate knowledge of the exact location of each of the muscles of the foot is not an absolute requisite, the nurse should, by reference to the illustrations, seek to familiarize herself with their names and their general location.

The anterior annular ligament consists of an upper vertical portion, which binds down the tendons as they descend downward to their several attachments (without this no power would be obtained) and a horizontal portion. This, with the internal and external annular ligaments, supports all the tendons, assisted by the fascia of the parts.

\section{MUSCLES OF THE PERINEUM}

The perineum is the anterior portion of the outlet of the pelvis. It is the space that lies between the anus and the genital organs. The muscles of this region are divided into two groups--those that control the organs of generation and those governing the urethra and the terminal portion of the rectum.

In the male the muscles of this region are: The accelerator urinæ, erector penis, transversus perinei, compressor urethræ, sphincter ani, levator ani, and coccygeus. 
In the female the muscles, while similar, are somewhat smaller than in the thale, and receive the following names: Constrictor vaginæ, erector clitoridis, transversus perinei, compressor urethræ, sphincter ani, levator ani, and coccygeus.

What are muscles?

\section{REVIEW QUESTIONS}

What peculiar property do muscles possess?

What is understood by origin and insertion?

How are muscles divided for purposes of description?

How many muscles are there in the body?

What is a sheath?

How are muscles attached to the skeleton?

What two kinds of muscular tissue have we in the body?

What muscle covers the upper part of the head?

How many muscles have we in the auricular region? Name them.

What muscles are in the palpebral region?

Name the several muscles of the orbital region.

How many pairs of muscles are there in the nasal region?

What do you understand by the superior maxillary region?

What peculiar action have the muscles of this region?

What muscles have we in the inferior maxillary region? Their action?

What important muscles have we in the intermaxillary region?

State the action of the several muscles in the temporomaxillary region.

How many muscles are there in the pterygomaxillary region?

What are the three large muscles of the neck?

What action has the digastric muscle?

What are the several functions performed by muscles of the palate region?

What is the pharynx?

What muscle elevates the pharynx?

What function do the pharyngeal muscles control?

What muscles control the movements of the head?

Into how many layers are the muscles of the back divided?

Name two of the superficial muscles of the back, and give their action.

Name the muscles of the abdomen.

What do you understand by the linea alba?

What important functions do the abdominal muscles assist in performing? 
Name three important muscles of the diaphragmatic region.

State the function of the several muscles just described.

What are the important muscles of the chest?

What function do they assist in performing?

What muscle forms the convexity of the shoulder?

What are the groups of muscles in the forearm?

How do the muscles of the forearm become inserted?

What are fasciæ? Explain their function.

Name the important muscles of the iliac region.

What is the longest muscle of the body? Where is it located?

Name three muscles of the gluteal region.

What muscle in this region arises by three digitations?

How many muscles are there in the leg?

What muscle forms the calf of the leg?

What is the function of the anterior annular ligament?

What is the perineum, and how many groups of muscles are there in this region? 


\section{C.H A P T E R I I I}

\section{THE CIRCULATORY OR VASCULAR SYSTEM}

THE organs of the circulatory or vascular system are the heart, the arteries, the veins, and all structures concerned in the propulsion or onward movement of the blood.

Circulation of the Blood.- The heart is the central station to which the blood is constantly returned, and from which it is as constantly distributed to all parts of the body. In making one complete revolution of the body the blood passes twice through this organ, making its circuit in the following manner:

As it returns from all parts of the system, the blood is emptied into the right auricle of the heart by what is termed the ascending and the descending vena cava; thence it passes into the right ventricle, the contraction of the heart propelling it through the pulmonary artery into the lungs. From this point the blood, oxygenated by its passage through the lungs, again returns through the four pulmonary veins (this is the only instance in the adult circulation where the veins carry arterial blood) into the left auricle, passing thence into the left ventricle; from which, by the contraction of the heart, it is propelled through the aorta and its numerous branches and distributed to every part of the body.

\section{THE HEART}

The heart is a hollow, muscular, pear-shaped organ, placed obliquely between the lungs. It is nearer the 
left than the right side of the thorax, its base pointing upward and its apex downward toward the left side. It is a strong body, being made up of involuntary muscular tissue, which is known as the myocardium. It is enclosed in the pericardium, a loose sac that also contains a serous lubricating fluid. The heart is commonly regarded as the starting-point of the blood in its course through the body; from this point the blood is propelled, through the arteries, to every part of the system.

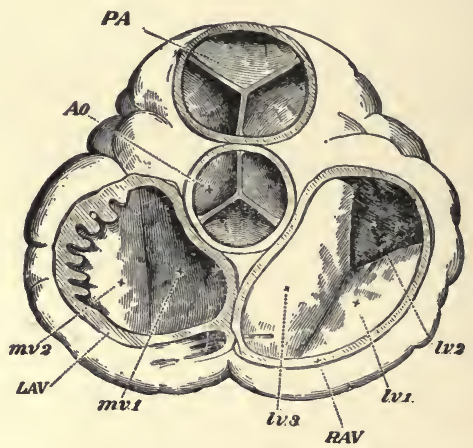

FIG. 45.-Orifices of the heart, seen from above, both the auricles and the great vessels being removed: $P A$, Pulmonary artery and its semilinar valves; $A 0$, aorta and its valves; $R A V$, tricuspid, and $L A V$, bicuspid valves; $m v$. segments of mitral valve; $l v$, segment of tricuspid valve (Huxley).

Cavities of the Heart.- The heart is divided into two parts-the right and the left heart. These are further divided into four distinct cavities, called respectively the right and the left ventricle and the right and the left auricle. By referring to the illustration (Fig. 48) it will be seen that the auricles are the upper cavities and the ventricles the lower.

The cavities of the heart are lined by a delicate, smooth membrane, the endocardium, which is continuous with the intima or innermost coat of the great blood-vessels. 
The right auricle is a little larger than the left, its walls are somewhat thinner, and its cavity is capable of containing about 2 ounces. The following openings are found in the right auricle: the superior vena cava, inferior vena cava, coronary sinus, foramina Thebesii.

The right ventricle is pyramidal in form, and extends from the right auricle to near the apex of the heart. Its anterior or upper surface is rounded and convex, and forms the larger part of the front of the heart. The right ventricle is provided with the opening of the pulmonary artery, and contains upon its interior the following valves: the tricuspid and semilunar, the latter guarding the pulmonary artery and the former the opening between the auricle and ventricle.

The left auricle resembles the right in having a principle cavity and an appendix, but its walls are thicker, measuring about one and one-half lines. It contains openings for the pulmonary veins, four in number, two emptying into the right and two into the left side of the auricle; frequently the latter terminate by one common opening.

The opening between the ventricle and auricle is provided with the bicuspid or mitral valves.

The left ventricle is longer than the right, and more conical in shape. It forms a small part of the left side of the anterior surface. It forms the apex of the heart from its projection beyond the right ventricle. Its walls are much thicker than those of the right side, the proportion being 3 to $\mathrm{I}$. It is thinner at the apex, and gradually gets thinner at the base. It is provided with the mitral valves and semilunar valves, the former making a division between the auricle and ventricle during contraction of the ventricle, and the latter guarding the aortic orifice. The aortic opening is to the right side.

The Valves.- On the right side, the valve which controls the opening from the right auricle to the right ven- 
tricle is known as the tricuspid valve. It is composed of three triangular segments, made up of a duplication of the endocardium, and continuous with fine cords called the * cordæ tendineæ, which close the aperture. The valve guarding the left side is composed of two irregular segments, and is called the bicuspid or mitral valve. If these valves were to become diseased, there would be a re-

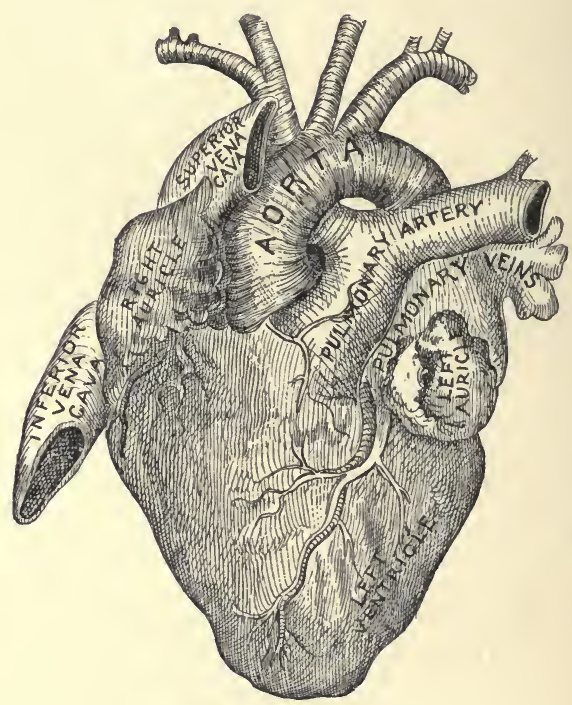

FIG. 46.-The heart (Stoney).

gurgitation of blood into the auricle (this will be clear to you if you will recollect the manner in which the blood passes through the heart, as previously explained). When healthy, the valves close accurately and form a complete partition between the auricle and the ventricle, and the blood is forced on through the large arteries.

Each auricle is provided with openings for the arteries and veins that enter from the circulation. The left 
ventricle gives off the aorta, the largest arterial structure in the body. The orifice of the aortic opening is guarded by valves, called the semilunar; in the fetus two are anterior and one posterior, but in the adult one is anterior and two are posterior, right and left.

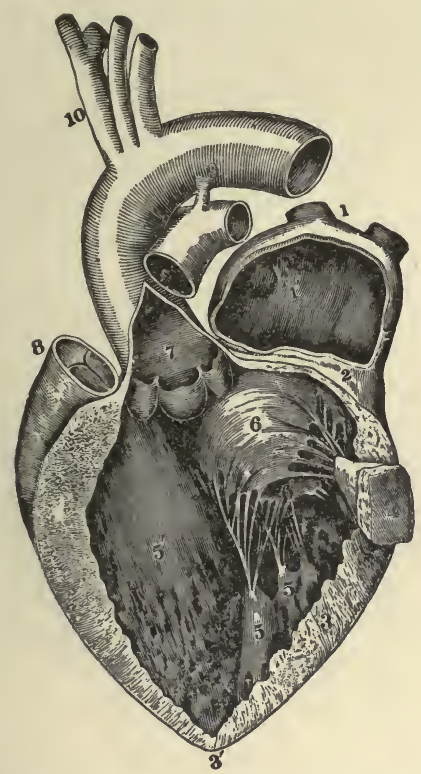

FIG. 47.-Left auricle and ventricle, opened and part of their walls removed to show their cavities: $\mathrm{I}$, Right pulmonary vein cut short $; \mathrm{r}^{\prime}$, cavity of left auricle; $3,3^{\prime}$, thick wall of left ventricle; 4 , portion of the same with papillary muscle attached; 5 , the other papillary muscles; $6,6^{\prime}$, the segments of the mitral valve; 7 , in aorta is placed over the semilunar valves; 8 , pulmonary artery; 10, aorta and its branches (Allen Thomson).

Contraction of the ventricles takes place, at the same time forcing the blood to the lungs from the right heart, through the pulmonary artery, and to the body generally from the left heart, through the aorta.

The aorta, rising from the left ventricle, immediately gives off, from its ascending portion, the two coronary 
arteries; at its arch it furnishes the left carotid and left subclavian on one side, and on the other only one, the innominate, which is larger than either of those on the left side. In the thorax the aorta gives off the bronchial arteries-one on each side; these nourish the lungs. It also gives off at this portion the esophageal, mediastinal,

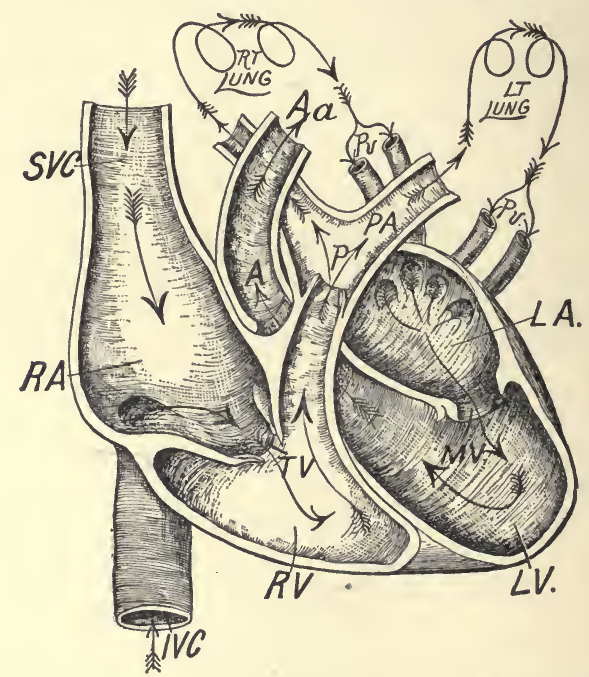

FIG. 48.-Normal blood-currents in the heart and relative position of the ventricles, auricles, and great vessels: $I V C$, Inferior vena cava; $S V C$, superior vena cava; $R A$, right auricle; $T V$, tricuspid valves; $R V$, right ventricle; $P$, pulmonary valves; $P A$, pulmonary artery ; $P v$, pulmonary veins; $L A$, left auricle; $M V$, mitral valves; $L V$, left ventricle; $A$, aortic valves; $A a$, arch of aorta (Page).

and intercostal arteries. From the abdominal portion it gives off the celiac axis with its three branches, the gastric, hepatic, and splenic arteries; the superior and inferior mesenteric, suprarenal, renal, spermatic (or ovarian), lumbar, and middle sacraI arteries. At the left side of the fourth lumbar vertebra the aorta bifurcates into two common iliacs, which each again divide into the internal 
and the external iliac, the internal supplying the pelvic tissues and the external the lower extremities.

The Heart=beat.-The heart in the healthy adult beats from 60 to 80 times in a minute; in children and infants the beat is more frequent, varying from roo to I 50 times in a minute.

Arteries are all characterized by a vibrating action, called pulsation, which corresponds to the working of the heart. This pulsation is in part dependent upon the action of the latter organ, but partly also on a similar power of contraction possessed by the arteries themselves.

The motion of the blood in the arteries is always in a direction away from the heart, and this fluid is propelled in these vessels by the pulsating force just described.

The systemic circulation is made up of two chief divisions - the arterial and the venous systems. In addition to these, two subsidiary systems are described-the pulmonary and the portal. We will describe first the arterial system, prefacing our remarks by a brief description of the arteries themselves.

This diagram, taken from Gray, shows the various parts and openings of the heart. The nurse will do well to make herself familiar with their names, and, by frequent reference to the illustrations, seek to learn their location:

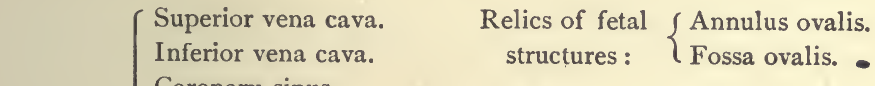

Openings Foramina Thebesii.

in the Auriculoventricular opening. Musculi pectinati.

heart: Pulmonary artery.

Pulmonary veins.

Aorta.

Auriculoventricular (right).

Valves (right side) $:\left\{\begin{array}{l}\text { Tricuspid. } \\ \text { Semilunar. }\end{array} \quad\right.$ Valves (left side) $:\left\{\begin{array}{l}\text { Mitral. } \\ \text { Semilunar. }\end{array}\right.$

Valves: $\left\{\begin{array}{l}\text { Eustachian. } \\ \text { Coronary. }\end{array}\right.$
} 


\section{THE ARTERIAL SYSTEM}

The arteries are the cylindric tubes that convey the blood from the ventricles of the heart to every part of the body. They are capable of preserving their form at all times. In ancient times, the arteries being found empty after death and still preserving their cylindric form, were believed to contain air. This theory was held until the time of Harvey, who discovered the circulation of the

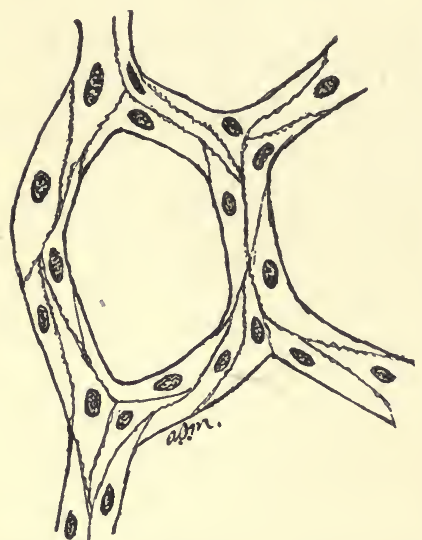

FIG. 49.-Diagram of capillaries (Morrow).

blood and presented it to the world as we now teach it.

As has been said, the main artery of the body is the aorta; from this all the arterial tissues derive their origin. This will be described further on. The communications of arteries are numerous. They do not terminate in veins, but are connected with the latter by an intermediate system of vessels, called capillaries (from capillus, a hair).

So numerous are these vessels that the slightest injury, such as a needle-prick, implicates many of these vessels and they bleed profusely.

The capillaries, as has been said, are the intermediate vessels between the arteries and veins. It is in these vessels that the most important functions or. changes of the blood occur - the change from arterial to venous blood. Here the phenomena of oxidation and calorification take place, and it is from this system that the endless variety of materials is supplied to the tissues of the body for nutrition and for glandular secretions. 
The Structure of Arteries.-The arteries are composed of three coats - an external, a middle, and an internal.

The external coat is firm and strong, and is made up of elastic and areolar tissues containing longitudinal and oblique fibers. It is of tougher texture than the middle coat.

The middle coat is a thick, circular coat which contains muscular tissue, the fibers of which are arranged in rings that vary in number according to the size of the artery. They contain some elastic tissue, but this is lost in the smaller arteries; in the larger ones the elastic tissue is blended with the areolar tissue.

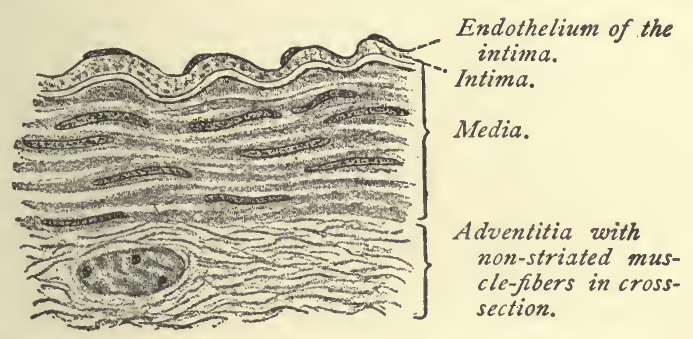

FIG. 50.-Section through human artery, one of the smaller of the mediumsized $\left(\times 6_{40}\right)$ (Böhm and Davidoff).

The internal coat is a thin, endothelial layer on the inside, and has an elastic exterior. In the large arteries there is an intermediate layer composed of areolar tissue.

From what has been said it will be seen that, considered from without inward, the external coat consists of areolar and elastic tissues; the middle coat, of smooth muscular fiber and areolar and elastic tissues; the internal coat, of elastic tissue and endothelium.

The walls of the capillaries are composed of only one coat, which is thin, transparent, and composed of tissue of light structure, which is lost in the elastic membrane of 
the internal coat of the arteries and in the middle coat of the veins. The capillaries form plexuses, which are very abundant, especially in the lungs, liver, kidneys, and other secreting glands. The skin and mucous membranes contribute very freely to this system. In the bones the capillaries are larger, but not so numerous.

The arteries are invested in a loose areolofibrous tissue called a sheath. This is considered an important structure in surgical operations; it also contains the accompanying veins, and at times a nerve.

The coats of the arteries are supplied with blood from the small branches which run in line with such structures. These are the vasa vasorum, or nutrient vessels, and are distributed to the external coat. The small veins which return the blood from the capillaries open into the small veins accompanying the arteries. The arteries are also supplied with nerves.

\section{THE AORTA}

The aorta is the largest arterial trunk in the body. It arises from the base of the left ventricle and middle of the heart, and at its beginning presents an enlargement, called the bulbus aortæ, which contains the semilunar valves. These valves are three in number, and prevent the blood from re-entering the left ventricle, whence it comes.

After leaving the left ventricle the aorta passes upward, backward, and over the root of the left lung to form the arch. On the right side this arch gives off a single branch about 2 inches in length; this is the innominate artery, which subdivides into the right common carotid and the right subclavian. On the left side of the arch the left common carotid and left subclavian are given off separately.

From the arch the aorta descends through the chest as 
the thoracic aorta; after passing through the diaphragm it is termed the abdominal aorta.

The first or ascending portion of the aorta is about $2 \frac{1}{2}$ inches in length, and is contained within the pericardium. Anteriorly, it is in relation with the pulmonary artery; on the left side, with the left auricle and pulmonary artery; on the right side, with the right auricle and superior vena cava; and behind, with the right pulmonary artery and veins.

The second or transverse portion gives off the innominate artery, left common carotid, and left subclavian arteries. It is in relation behind with the left recurrent nerve, trachea, esophagus, thoracic duct, and nerves of the cardiac plexus; in front, with the left phrenic, left superior cardiac, and left pneumogastric nerves.

The third or descending portion of the arch lies adjacent to the third dorsal vertebra, and is covered with a portion of the pleura.

The descending aorta proper is divided into two great portions, named the thoracic and the abdominal aorta.

The thoracic aorta takes its origin at the lower border and to the left of the third dorsal vertebra, curves forward to the right, runs downward on the median line of the vertebral column, and passes through the aortic opening in the diaphragm. Relations: behind, with the vertebral column and vena azygos minor; anteriorly, with the esophagus and left pneumogastric nerve; on the left, with the pleura; on the right, with the thoracic duct.

The abdominal portion (see Fig. 56) enters through the opening in the diaphragm, proceeds downward in line with the vertebral column, slightly to the left, and extends to the fourth lumbar vertebra, where it divides into the two common iliac arteries. Relations: in front, with the left renal and splenic veins, pancreas, transverse duodenum, and mesentery; behind, with the thoracic duct, receptaculum chyli, and left lumbar veins; on the left, with the left 
semilunar ganglion, suprarenal capsule, and sympathetic nerve; on the right, with the right semilunar ganglion and the commencement of the vena azygos major.

The branches given off from the aorta are as follows:

$$
\text { Arch : }\left\{\begin{aligned}
\text { Ascending portion. } & \text { Coronary, right and left. } \\
& \text { Innominate }:\left\{\begin{array}{l}
\text { Right common carotid. } \\
\text { Right subclavian. }
\end{array}\right. \\
\text { Transverse portion. } & \left\{\begin{array}{l}
\text { Left common carotid. } \\
\text { Left subclavian. }
\end{array}\right.
\end{aligned}\right.
$$$$
\text { Thoracic aorta : }\left\{\begin{array}{l}
\text { Pericardiac. } \\
\text { Bronchial. } \\
\text { Esophageal. } \\
\text { Posterior mediastinal. } \\
\text { Intercostal. }
\end{array}\right.
$$$$
\left\{\text { Celiac axis : } \left\{\begin{array}{l}
\text { Gastric. } \\
\text { Hepatic. } \\
\text { Splenic. }
\end{array}\right.\right.
$$$$
-
$$$$
\text { Inferior phrenic (2) suprarenal. }
$$

Abdominal aorta :

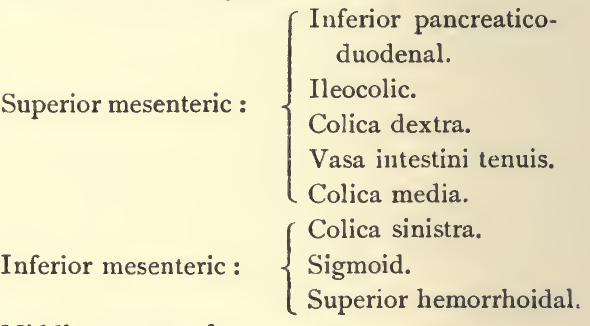

Middle suprarenal.

Renal.

Spermatic (in female, ovarian).

Lumbar.

Dorsal.

Middle sacral.

The coronary artery arises from the aortic sinuses at the commencement of the ascending portion at the arch, immediately above the margin of the semilunar valves, dividing into the right and the left coronary arteries, which inosculate with each other and supply the substance 
of the heart with nutrition. These arteries encircle the heart in both the horizontal and the vertical directions.

The innominate artery is the first and largest trunk given off from the arch of the aorta; it is about $\mathrm{I} \frac{1}{2}$ inches in length, and ascends obliquely toward the right sternoclavicular articulation, where it divides into the right common carotid and right subclavian arteries. It occasionally gives off a small branch to the thyroid gland.

The Common Carotid Arteries.-The right arises from the bifurcation of the innominate artery, opposite the right sternoclavicular articulation. The left ascends from the arch of the aorta. The right is shorter than the left, more anterior, and somewhat larger.

The right common carotid ascends the neck perpendicularly by the side of the trachea and larynx, from behind the right sternoclavicular articulation to the level of the upper border of the thyroid cartilage, where it divides into the right external and internal carotids.

The left common carotid ascends from the aortic arch obliquely outward to the side of the neck, and upward by the side of the trachea and esophagus to the upper border of the thyroid cartilage, where it divides into the left external and internal carotids.

The external carotid ascends perpendicularly between the lower jaw and auditory meatus, where it divides into two terminal branches, the temporal and the internal maxillary; it gives off the following branches:

Anterior.

Posterior.

Superior thyroid.

Occipital.

Lingual.

Posterior auricular.

Facial.

Ascending.

Ascending pharyngeal.
Terminal. Superficial temporal. Internal maxillary. 
The external branches arise from the commencement of the external carotid, within a short distance of each other. The lingual and facial sometimes bifurcate from the common carotid.

The superior thyroid artery arises from the external carotid; and is distributed to the thyroid gland; it anastomoses with the vessel of the opposite side, and gives off the following branches:

Hyoid.

Superior laryngeal.
Cricothyroid.

Muscular.

The lingual artery ascends obliquely from its origin parallel with the hyoid bone, and directly forward, taking the name ranine artery, where it inosculates with the opposite side; it gives off the following branches: hyoid, dorsalis linguæ, sublingual, and ranine.

The facial artery arises immediately above the lingual, and a short distance above the great cornu of the hyoid bone. It is embedded in the submaxillary gland, curves around the lower jaw at the inferior angle of the masseter muscle, ascends to the angle of the mouth, and terminates at the inner angle of the eye, under the name of the angular artery.

The facial artery is tortuous in its course over the buccinator muscle, to accommodate itself to the movements of the jaw. The branches that are given off are divided into two sets-those given off below the jaw (cervical) and the facial; these are as follows:

Below the jaw:

Inferior palatine.

Tonsillar.

Submaxillar.

Submental.

Muscular.
On the face:

Buccal (muscular).

Inferior labial.

Inferior coronary.

Superior coronary.

Lateralis nasi.

Argular: : 
In its distribution this artery sometimes terminates at the angle of the mouth or nose, at the ala, and its inosculations are numerous.

The occipital artery, one of the branches of the external carotid, arises opposite the styloid process of the temporal bone, and ascends beneath the parotid gland and under

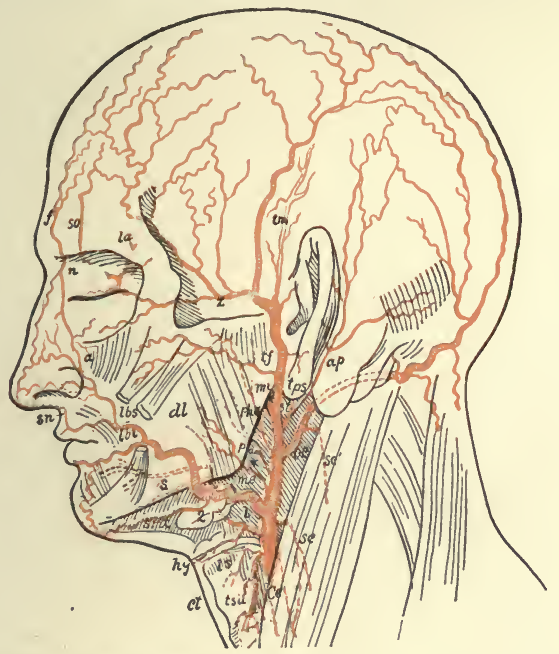

FIG. 51.-Facial and temporal arteries: $C e$. External carotid; $t s u$, superior thyroid; $l s$, superior laryngeal; $c t$, cricothyroid; $h y$, hyoid branch of lingual; $d l$, dorsal of tongue; $s$, sublingual; me, facial; smt, submental; $a$, angular; $l b i$, inferior labial; $l b s$, coronary of upper lip; $s u$, artery of nasal septum ; $s c, s c^{\prime}$, sternomastoids ; $a p$, posterior auricular ; st, stylomastoids; $p d a$, ascending pharyngeal; $m i$, internal maxillary; $t p s$, superficial temporal ; $t f$, transverse facial ; $t m$, middle temporal; $z$, external supra-orbital; $f$, frontal ; so, supra-orbital ; la, lacrimal ; $n$, nasal; *, parotid branches of - external carotid (after Henle).

the sternomastoid muscle, passing along to the occipital groove in the mastoid portion of the temporal bone; it then ascends and is distributed to the back part of the head. It furnishes the following branches: muscular (supplying the sternomastoid, digastric, stylohyoid), auricular, meningeal, and arteria princeps cervicis. 
The sternomastoid (superficial descending branch) is distributed to the sternomastoid muscle and lymphatic glands.

The posterior auricular artery arises from the external carotid opposite the styloid process of the temporal bone. It is distributed to the external ear and side of the head; it anastomoses with the occipital and temporal arteries. It gives off several branches to the muscles and furnishes a branch to the parotid gland, as well as branches to the mastoid cells of the temporal bone and tympanum of the ear.

The ascending pharyngeal artery is the smallest of the branches of the external carotid. Its branches are divided into three sets-meningeal, to the dura mater; pharyngeal, to the pharynx, tonsils, soft palate; and a prevertebral branch, to the posterior part of the throat.

The parotid arteries give off several branches that supply the parotid gland; they also supply the integument of the face and the masseter muscle.

The temporal artery (superficial) is one of the terminal branches of the external carotid, and divides into anterior and posterior branches. The anterior branch runs forward and is distributed to the temple; the posterior anastomoses with the posterior auricular; with the occipital, and with its fellow on the opposite side. The branches given off from this artery are: the anterior auricular, which are distributed to the pinna of the ear; an orbital branch, which unites with the ophthalmic artery; the transverse facial, which arises from the temporal and unites with the facial and infra-orbital arteries; and the middle temporal, which supplies the temporal muscle, associated with the deep temporal artery.

The internal maxillary artery is the larger terminal branch of the external carotid; it passes inward in the deep structure of the face, at the inner side of the condyle of 
the lower jaw, and divides into three portions - the maxillary, the pterygoid, and the sphenomaxillary; these are subdivided into the following branches (see Fig. 51):

Naxillary.

Tympanic.

Inferior dental : $\quad\left\{\begin{array}{l}\text { Mylohyoid. } \\ \text { Incisor. } \\ \text { Mental. }\end{array}\right.$

Middle meningeal.

Small meningeal.

Pusterior dental.

Infra-orbital.
Pterygoid.

Deep temporal :
Pterygoid. Masseteric.

Buccal.

Sphenomaxillary.

Sphenopalatine.

Pterygopalatine.
Posterior palatine.

Vidian.

The Internal Carotid Artery.-This arises from the common carotid, curves backward and outward, and ascends upward by the side of the pharynx to the carotid foramen in the petrous portion of the temporal bone. It then passes inward along the carotid canal, where it pierces the dura mater and divides into three terminal branches. This artery makes many curves, which may be seen at the cervical portion, at the base of the skull. It divides into the anterior and middle cerebral arteries. The cervical portion of the artery gives off no branches. Within the skull the following branches are given off:

Tympanic.

Arteriæ receptaculi.

Anterior meningeal.

Ophthalmic.
Anterior cerebral.

Middle cerebral.

Posterior communicating.

Anterior choroid.

The tympanic is a small branch given off in the carotid canal; it penetrates the tympanum and unites with the tympanic branch of the internal maxillary and the stylomastoid arteries.

The arteriæ receptaculi are small vessels that supply the 
cavernous and inferior petrosal sinuses, pituitary body, and Gasserian ganglion.

The anterior meningeal supplies the dura mater of the anterior fossa.

The ophthalmic artery arises from the cavernous portion, enters the orbit by the optic foramen, dividing at the inner angle of the eye into two terminal divisions- the frontal and the nasal-which further divide into the following branches:

Orbital: $\left\{\begin{array}{l}\text { Lacrimal. } \\ \text { Supra-orbital. } \\ \text { Posterior ethmoid. } \\ \text { Anterior ethmoid. } \\ \text { Palpebral. } \\ \text { Frontal. } \\ \text { Nasal. }\end{array} \quad\right.$ Ocular: $\left\{\begin{array}{l}\text { Muscular. } \\ \text { Anterior ciliary. } \\ \text { Short ciliary. } \\ \text { Long ciliary. } \\ \text { Arteria centralis retinæ. }\end{array}\right.$

The anterior cerebral artery enters the longitudinal fissure between the two hemispheres of the brain; it gives branches to the olfactory and optic nerves. The two arteries on each side join, shortly after origin, by a short anastomosing branch. (See chapter on the Nervous System for a more complete description of the arteries that supply the brain, including the middle cerebral, posterior communicating, and anterior choroid.)

The Subclavian Artery.-The right subclavian artery takes it origin from the innominate artery, opposite the sternoclavicular articulation; the left arises directly from the aorta. At the lower border of the first rib it ceases to be called the subclavian, and becomes the axillary. For descriptive purposes the artery is generally divided into three sections. This division is necessary, owing to the fact that there is a difference in the origin of the right and the left arterial trunk; it follows, therefore, that there is a marked difference in the first part of the course of this artery. On the right, the first portion passes 
obliquely upward and outward to the inner border of the scalenus anticus muscle; on the left side it passes upward to the inner border (not outward). The second portion curves outward behind the same muscle; the third passes downward and outward beneath the clavicle to the lower border of the first rib, where it becomes the axillary artery.

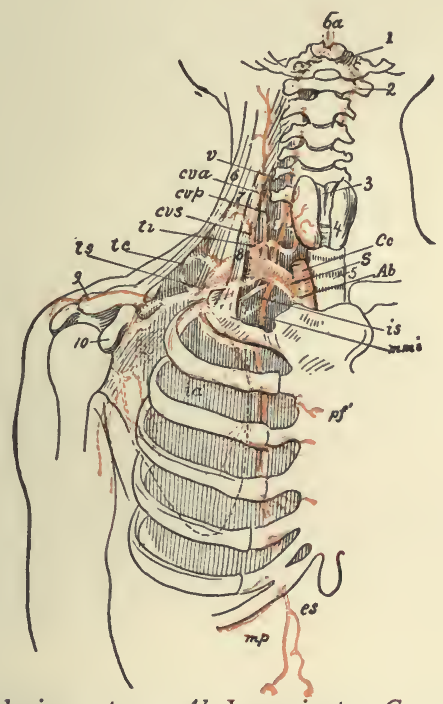

FIG. 52.-Subclavian artery: $A b$, Innominate; $C c$, common carotid; $S$, subclavian; $v$, vertebral ; $b a$, basilar; $m m i$, internal mammary ; $e s$, superior epigastric ; $m p$, musculophrenic ; is, superior intercostal; cvp, deep cervical ; $t i$, inferior thyroid; $c v a$, ascending cervicai; $c v s$, superficial cervical; $t s$, suprascapular; $t c$, posterior scapular (after Herle).

The branches which this important artery furnishes are the following:

Vertebral.

Internal mammary.

Thyroid axis :

$\left\{\begin{array}{l}\text { Inferior thyroid. } \\ \text { Suprascapular. } \\ \text { Transversalis colli. }\end{array}\right.$

Superior intercostal. 
The vertebral artery is the first and largest branch. It arises from the back part of the subclavian artery, passes upward through the foramina in the transverse process of the upper six cervical vertebræ; it turns backward around the articulating process of the atlas, after which it enters the skull through the foramen magnum. The two vertebral arteries unite to form the basilar artery. The branches of the vertebral may be divided into two groups:

Cervical branches.

Lateral spinal.

Muscular.
Cranial branches.

Posterior meningeal.

Anterior spinal.

Posterior spinal.

Postero-inferior cerebellar.

The basilar artery is situated at the base of the brain. It runs in the median groove of the pons Varolii, and divides into the following branches:

Transverse.

Superior cerebellar.

Antero-inferior cerebral. Posterior cerebral.

The internal mammary artery arises and descends within the chest from the under side of the subclavian, alongside of the sternum, upon the cartilages of the ribs, to the sheath of the rectus muscle, where it takes the name of the superior epigastric; the continuation of this vessel joins the epigastric branch of the external iliac. This artery is crossed by the phrenic nerve just as it enters the chest. It distributes to the muscles of the abdominal walls and gives off several branches, which are as follows:

Comes nervi phrenici.

Mediastinal.

Pericardiac.

Sternal.
Anterior intercostal.

Perforating.

Musculophrenic.

Superior epigastric. 
The thyroid axis is a short trunk, and divides into three branches - the inferior thyroid, suprascapular, and transversalis colli.

The superior intercostal artery arises from the subclavian, and gives off branches to the intercostal spaces and to the muscles of the dorsal region; it runs behind the pleura and to the outer side of the sympathetic nerve, and joins the intercostal branch from the aorta.

The profunda cervicis is a branch of the superior intercostal; it unites with branches from the occipital and vertebral arteries, and supplies the several muscles of the parts through which it passes.

\section{The Axillary Artery.-} We will now consider the axillary artery and its branches. This artery commences at the lower border of the first rib and extends

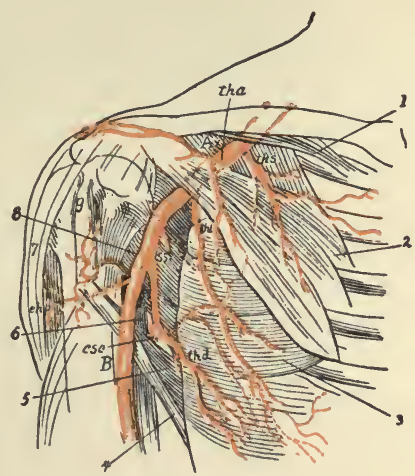

FIG. 53.-Axillary artery and branches: $A x$, Axillary ; $B$, brachial ; ths, superior thoracic; tha, acromiothoracic; thl, external mammary; cha, anterior circumflex; chp, posterior circumflex; ss, subscapular; csc, circumflex of scapula; 1 , subclavius muscle; 2 , pectoralis muscle ; 3 , serratus magnus; 4, latissimus dorsi ; 5 , teres major; 6 , long head of triceps; 7 , deltoid; 8 , subscapular muscle; 9, bicipital groove (after Henle).

to the lower border of the teres major muscle, where it becomes the brachial. Its relations are as follows:

\section{In front.}

Pectoralis major.

Pectoralis minor.
Inner side.

First intercostal muscle.

Serratus magnus.

Brachial plexus.

Axillary vein.
Outer side.

Brachial plexus.

Coracobrachialis. 
The branches are:

Superior thoracic.

Acromial thoracic.

Long thoracic.
Thoracica alaris.

Subscapular.

Anterior circumflex.

Posterior circumflex.

The Brachial Artery. -This is a continuation of the axillary, passing down the inner side of the arm to a little

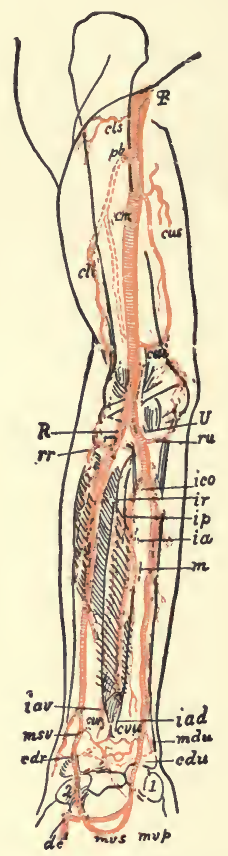

FIG. 54.-Brachial artery and branches: $B$, Brachial; $R$, radial; $U$, ulnar; $c l s$, deltoid artery ; $c l i$, deep radial ; $p b$, superior profunda ; $c m$, arteria collateralis media; cui, anastomotic; $r r$, anterior radial recurrent; $r u$, anterior ulnar recurrent; ico, common interosseous; $i a$, anterior interosseous ; $i p$, posterior interosseous; ir, posterior interosseous recurrent; iar, palmar anterior interosseous; $i a d$, dorsal anterior interosseous; $m$, median; $c v r$, anterior radiocarpal; $c v u$, anterior ulnocarpal; $c d r$, posterior radiocarpal; $c d u$, posterior ulnocarpal ; $m d u$, posterior ulnocarpal ; $m v s$, superficial volar ; $m v p$, ulnar part of deep palmar arch; $d c$, first palmar digital (after Henle). 
below the bend of the elbow, where it divides into radial and ulnar arteries. It gives off the following branches:

Superior profunda.

Nutrient.

Inferior profunda.

Anastomotica magna.

Muscular.

The radial artery is a branch of the brachial, and appears to be a continuation of this artery; its course is along the radial side of the forearm from the elbow to the wrist; passing backward around the thumb, it runs be-

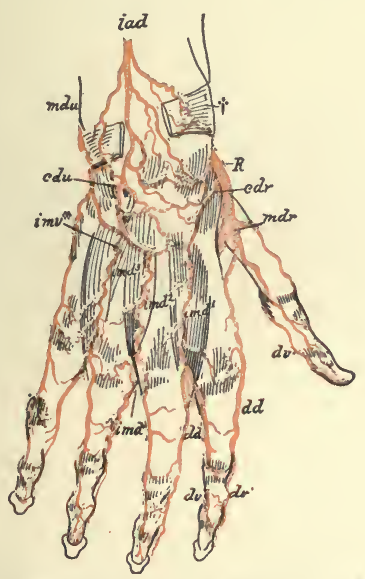

FIG. 55.-Dorsal arteries of hand : $c d r$, Radial posterior carpal ; $c d u$, ulnar posterior carpal ; $m d r$, dorsales pollicis ; $i m d^{1}$, $i m d^{2}$, $i m d^{3}$, dorsal interossei ; imv*, posterior perforating; $d d$, dorsal digital (after Henle).

tween the heads of the first dorsal interosseous muscle into the palm of the hand, forming the deep palmar arch.

For descriptive purposes this artery is divided into three parts, which correspond with the parts which it 
supplies, viz., the forearm, the wrist, and the hand. Its divisions are:

Forearm.

Radial recurret.

Muscular.

Superficialis volæ.

Anterior carpal.
Wrist.

Posterior carpal.

Metacarpal.

Dorsales pollicis.

Dorsales indicis.
Hand.

Princeps pollicis.

Radialis indicis.

Interosseous.

Perforating.

Palmar recurrent.

The ulnar artery is larger than the radial, and runs obliquely inward to the middle of the forearm; then across the annular ligament to the radial side and to the palm, where it unites with the superficialis volæ or radialis indicis to form the superficial palmar arch. The branches of the ulnar artery are as follows:

\section{Forearm.}

Anterior ulnar recurrent. Posterior ulnar recurrent. Interosseous (anterior and posterior).

Muscular.
Wrist.

Anterior carpal. Posterior carpal.

Hand.

Communicating.

Digital.

Superficial palmar arch. Deep palmai arch.

Having considered the arterial structure of the upper portion of the body, we will now describe the blood-supply of the trunk and lower extremities, beginning with the thoracic aorta.

\section{THE THORACIC AORTA}

The thoracic aorta commences at the left side of the lower border of the fourth dorsal vertebra; it terminates at the aortic opening in the diaphragm, in front of the last dorsal vertebra.

Its branches are as follows:

Pericardiac.

Bronchial.
Esophageal.

Posterior mediastinal. 
The pericardiac arteries are small and irregular, and are distributed to the pericardium.

The bronchial arteries are three in number-one for the right lung and two for the left; they are distributed to the lung-tissue, and also provide small branches that supply the bronchial glands, esophagus, and pericardium.

The esophageal arteries number five or six; they are given off from the anterior part of the aorta, and are distributed to the esophagus, forming a chain of arterial supply about it. They anastomose freely with the arteries in the vicinity.

The posterior mediastinal arteries are small, and are distributed to the lymphatic glands and cellular tissue of the posterior mediastinum.

The intercostals (posterior) arise from the posterior part of the aorta, and number ten on each side. The first intercostal space is supplied by the superior inter= costal artery, a branch of the subclavian. They anastomose freely with the anterior intercostal, mammary, axillary, epigastric, phrenic, and lumbar arteries. They supply the vertebræ, spinal cord, dorsal muscles, and skin.

\section{THE ABDOMINAL AORTA}

This important structure has been briefly described in another section (page 97). For the purpose of making the nurse more familiar with this artery and its branches, a more complete description will now be given.

The abdominal aorta commences at the aortic opening in the diaphragm, in front of the body of the last dorsal vertebra; descending to the left of the vertebral column, it terminates on the body of the fourth lumbar vertebra by dividing into the right and left common iliac 
arteries. This portion of the aorta gives off the following branches:

Phrenic.

Celiac axis : $\begin{cases}\text { Gastric. } & \text { Inferior mesenteric. } \\ \text { Hepatic. } & \text { Suprarenal. } \\ \text { Splenic. } & \text { Renal. }\end{cases}$
Superior mesenteric. $\begin{aligned} & \text { Lumbar. } \\ & \text { Spermatic (ovarian in female). }\end{aligned}$

The phrenic arteries supply the under surface of the diaphragm. Each gives off a superior suprarenal branch.

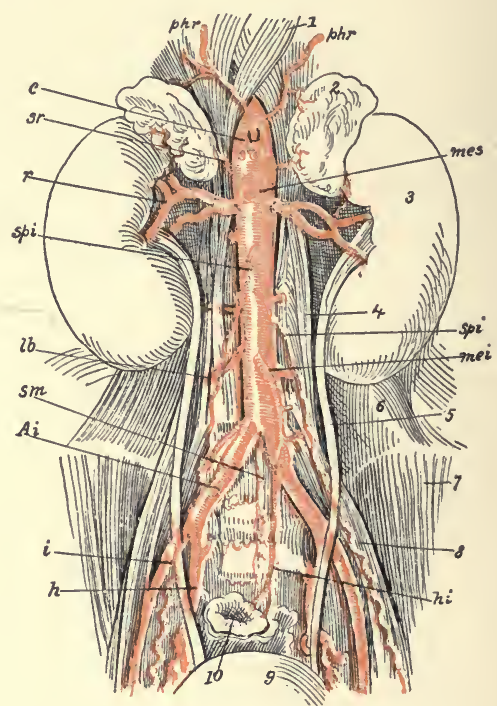

FIG, 56.-The abdominal aorta: $A i$, Common iliac ; $i$, external iliac; $h$, internal iliac; $s m$, middle sacral ; phr, inferior phrenic; $l b$, lumbar ; $c$, celiac ; mes, superior mesenteric ; mei, inferior mesenteric ; $s r$, suprarenal ; $r$, renal ; $s p i$, spermatic; $h i$, internal hemorrhoidal (after Henle).

The celiac axis arises from the aorta opposite the margin of the diaphragm, passes out for $\frac{1}{2}$ inch, and divides into three branches-the gastric, hepatic, and splenic. 
The gastric (sometimes called the coronary) passes along the lesser curvature of the stomach, anastomosing with the esophageal, splenic, and hepatic arteries.

The hepatic artery passes along the border of the lesser omentum to the liver, where it gives off the following branches: Pancreatic, pyloric, gastroduodenalis, and cystic. These are distributed to the parts indicated by the names, and anastomose with the splenic, gastric, and superior mesenteric arteries.

The splenic is the largest of the three branches of the celiac axis. It passes to the left along the upper border of the pancreas, and divides into five or six branches, which enter the spleen at its hilum and are distributed to the structure of the spleen. This is what is called a serpentine artery, since it pursues an extremely tortuous course. It has the following branches:

Pancreaticæ parvæ.

Pancreatica magna.
Vasa brevia.

Gastro-epiploica sinistra.

The superior mesenteric artery is given off from the abdominal aorta about $\frac{1}{4}$ inch below the celiac axis, and passes downward and forward from left to right; it is distributed to the small intestine, with the exception of the first part of the duodenum. It gives off five branches:

Inferior pancreaticoduodenal.

Vasa intestini tenuis. .
Ileocolic.

Colica dextra.

Colica media.

The inferior mesenteric artery is given off from the aorta about 2 inches above the bifurcation, passing to the left and supplying the descending colon, sigmoid flexure, and part of the rectum, anastomosing with the colica media, and distributing branches to the transverse colon. It gives off the following branches: colica sinistra, sigmoid, and superior hemorrhoidal; these furnish nutri- 
tion to the pelvic tissues and rectum, and freely anastomose with arteries therein contained.

The suprarenal arteries are two small branches that are distributed to the suprarenal capsule of the kidney.

The renal arteries are two large branches given off $\because$ from the abdominal aorta, the right being longer and lower than the left. Before entering the kidney. they divide. into several branches which are distributed to the substance of the organ. Each vessel gives off small branches -one to the ureter and the other to the suprarenal capsule.

The spermatic arteries (ovarian in the female) are given off in front of the abdominal aorta on each side, below the renals, and pass behind the peritoneum. In the male they pass out through the inguinal canal to the testicles; in the female they run between the layers of the broad ligament to reach the ovary, Fallopian tubes, and uterus. In their course they supply the several organs mentioned.

The lumbar arteries are usually five in number on each side; they pass about the lumbar vertebræ, and divide into two branches-one dorsal and one abdominal -which are distributed to the muscles of the back, the vertebræ, the spinal cord, and the abdominal muscles.

The sacra media arises from the bifurcation of the abdominal aorta, passes along the anterior part of the sacrum to the coccyx, and supplies branches to the rectum and anterior sacral nerves; it anastomoses with the lateral sacral arteries.

The Common Iliac Arteries.-As has been said, the abdominal aorta divides into the common iliac arteries:These extend from the bifurcation, which takes place usually at the fourth or the fifth lumbar vertebra. These arteries are about 2 inches long, and pass outward and 
downward to the pelvis opposite to the sacro-iliac symphysis, where they divide into the internal and the external iliac. The right artery is longer than the left. In the female the bifurcation is more expanded than in the male.

The Internal Iliac Artery.-This is a short branch from the common iliac, extending to the sacrosciatic foramen, where it divides into two branches-the anterior and the posterior-which subdivide into the following:

Anterior.

Superior vesical.

Middle vesical.

Inferior vesical.

Middle hemorrhoidal.

Uterine.

Vaginal.

Obturator.

Sciatic.

Internal pudic.
Posterior.

Iliolumbar.

Lateral sacral.

Gluteal.

The external iliac artery passes along the psoas muscle to Poupart's ligament. A line drawn from the umbilicus to the middle of Poupart's ligament indicates its course. In addition to branches to the psoas muscle and to the neighboring lymphatic glands, it gives off the deep epigastric and the deep circumflex iliac.

The Femoral Artery.-When the external iliac passes under Poupart's ligament it becomes the femoral. From this point it passes down the thigh. A line drawn from the mid-point between the anterior superior spine of the ilium and the symphysis pubis to the inner side of the internal condyle of the femur indicates its course. 'Together with its vein it is enclosed in a strong sheath, but is separated from the vein by a partition of fibrous septum. 
It is divided into a superficial and a deep branch, which are further subdivided into the following branches:

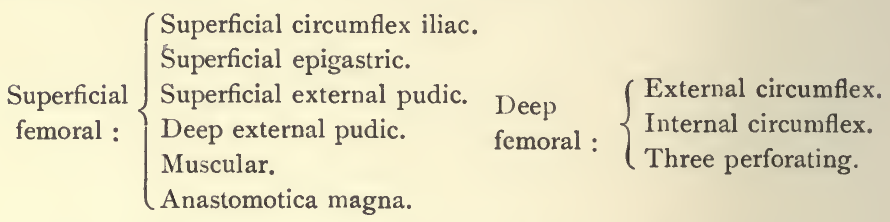

The popliteal artery commences at the opening in the adductor magnus, and, passing downward behind the knee-joint, it divides at the lower border of the popliteus muscle into the anterior and the posterior tibial. The walls are exceedingly thick, thus protecting the artery, which is subjected to strain from the pressure that is applied from without, as in flexing the limb on the thigh. It is the deepest structure in the popliteal space. The branches which it gives off are as follows:

Superior muscular.

Inferior muscular or sural.

Cutaneous.

Azygos articular.
Superior external articular. Superior internal articular. Inferior internal articular. Inferior external articular.

The anterior tibial artery passes through the interosseous membrane to the anterior tibial region, and runs down the anterior part of the leg to the ankle-joint, where it becomes the dorsalis pedis. The branches are the recurrent, muscular, external malleolar, and internal malleolar.

The dorsalis pedis artery is on the tibial side of the dorsum of the foot, passing from the ankle to the base of the metatarsal bone of the great toe, where it gives off a branch-the dorsalis hallucis; it then passes to the sole of the foot, between the heads of the interosseous muscle, and completes the plantar arch by anastomosing with the external plantar artery. Its branches are the tarsal, 


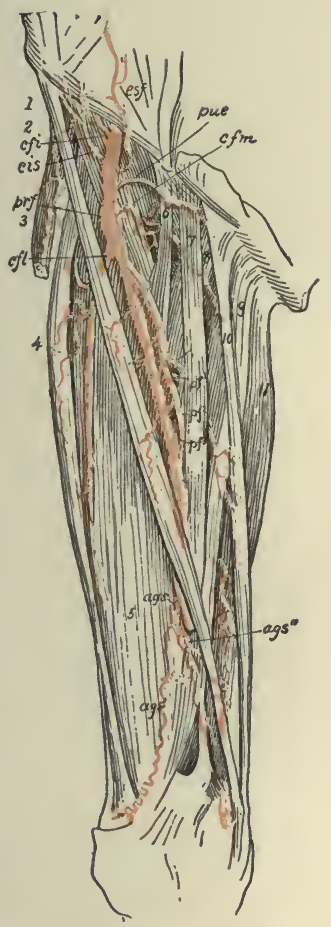

FIG. 57.-Femoral artery : $c f i$, Common femoral; esp, superficial epigastric ; cis, superficial circumflex iliac; pue, external pudic; prf, deep femoral; $c f l$, external circumflex of thigh ; $c \mathrm{fm}$, internal circumflex of thigh; $\not f^{1}$, first perforating; $p f^{2}$, second perforating; $p f^{3}$, third perforating; ags, anastomotica magna; $s f$, superficial femoral (after Henle).

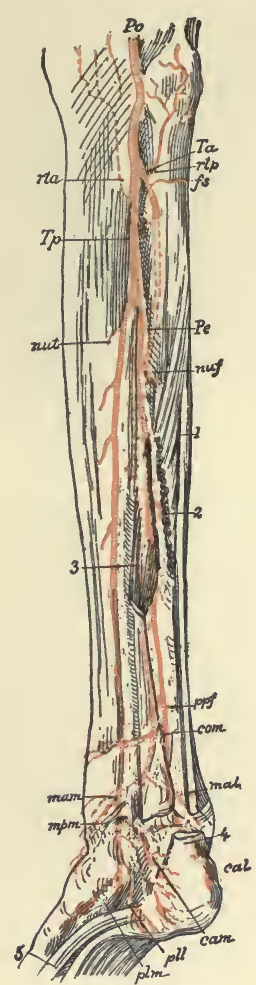

FIG. 58.-Posterior tibial artery : $P o$, Popliteal; $T a$, anterior tibial; $T p$, posterior tibial; $P e$, peroneal; $r t a$, anterior tibial recurrent; $r t p$, posterior tibial recurrent; $f s$, superior fibular; nuf, nutrient of fibula; nut, nutrient of tibia; ppf, anterior peroneal; com, transverse anastomotic ; mam, anterior medial malleolar; mal, internal malleolar; $m p m$, posteromedian malleolar; cam, middle calcaneal ; plm, internal plantar; pll, external plantar (after Henle).

metatarsal, interosseous, dorsalis hallucis, and communicating. 
The posterior tibial artery passes down the tibial side of the leg, from the lower border of the popliteal muscle to the concavity of the os calcis, where it divides into the internal and the external plantar arteries. The branches of the posterior tibial are as follows: Pero-

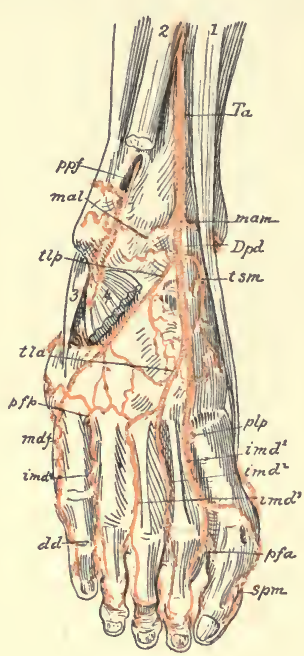

FIG. 59.-Dorsal arteries of foot : $T a$, Anterior tibial; $D p d$, dorsal; $p p f$, anterior peroneal ; mam, anteromedian malleolar; mal, internal malleolar; $t s m$, internal supratarsal; $t l p$, external tarsal; tla, metatarsal; plp, deep plantar; $i m d^{1}$, $i m d^{2}$, $i m d^{3}$, the dorsal interossei ; $\not f p$, posterior perforating; $p f a$, anterior perforating; $s p m$, digital branch of communicating (after Henle). neal, nutrient, muscular, internal calcanean, internal plantar, and external plantar.

The Pulmonary Artery. - The pulmonary artery arises from the right ventricle of the heart, in front of the aorta, and is divided into a right and a left pulmonary artery, which pass to the lungs. The right is larger than the left. These vessels transmit the venous blood to the lungs, to be supplied with oxygen. 'They will be subsequently more fully described.

\section{THE VENOUS SYSTEM}

The venous system is that part of the systemic circulation which returns the venous blood to the right auricle of the heart.

The veins take their origin from absorbent radicles from the capillaries, which in their course unite and reunite, thus forming larger and still larger trunks, until they finally form two large vessels-the ascending and the descending vena cava. The circulation in the veins is always in a direction toward the heart. 
There is no pulsation in veins, as there is in the arteries, hence the circulation in them depends upon a different mechanism. They are supplied with numerous valves (Fig. 60), which prevent any reflux of blood, and close in such a manner that the blood can flow only toward the heart.

Physiologists are not yet in accord regarding all the theories that have been proposed to account for the grand principle of venous circulation, or the power of sustaining it. The valvular arrangement just mentioned at once suggests that an alternate contraction and relaxation is exercised upon them; such action, we can readily perceive, is afforded to some extent by the movements of the muscles, but this action is not sufficiently regular to account for the regularity of the venous circulation. Another action, however, that is more regular and universal, is that of pulsation of the
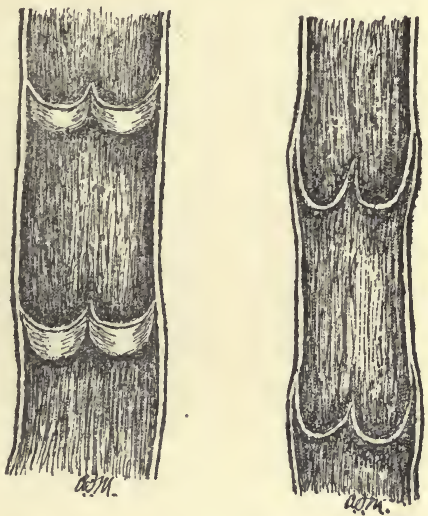

FIG. 60.-Diagram of the valves of veins (Morrow). arteries. The swell of the

arteries in the diastolic motion produces great pressure in every part of the system. We have a very striking example of this in the throbbing pain in the head in some forms of fever, or in cases of inflammation, especially when the parts are much swollen.

Thus the impulse of the heart and arteries not only propels the blood through the arteries, but tends indirectly also to force it forward in the veins.

There is, however, another physical principle which 
is cited as an agency in venous circulation, and that is capillary attraction. Still another factor responsible for the return of the venous blood to the heart is furnished by thoracic aspiration. The following illustrations of the venous system give a fair conception of the course of the veins and shows their relation to the arterial system.

The blood is of much darker color in the veins than in the arteries. This change takes place during the passage of the blood through the capillary system, and is dependent upon the phenomena of combustion and the general metamorphosis of the materials of the tissues, all of which are in contact with the capillary system.

The veins of the body are divided into two groups: those that pass by way of the superior and those that empty into the inferior vena cava. The blood from the lower extremities is brought by way of the inferior vena cava; the blood from the brain, neck, and face passes through the internal and the external jugular veins, which unite with the venous trunk from the upper extremity to form the superior vena cava.

The external jugular vein starts at the angle of the lower jaw and terminates in the subclavian vein.

The internal jugular vein passes downward in line with the common carotid, and unites with the subclavian to form the innominate vein.

The subclavian vein receives the blood from the arm. The veins, with the exception of the superficial branches, accompany the arteries in the same sheath. The right and left azygos veins receive the blood from the spine.

The innominate veins unite in forming the superior vena cava. The inferior vena cava is formed by the union of the two common iliacs, which receive all the blood from the deep and the superficial veins of the lower limbs. The large vein of the leg is called the internal saphenous. 
Sinuses are venous channels found chiefly within the cranium. They, together with the blood-supply of the cerebrum, will be considered in the chapter on the Nervous System.

\section{VEINS OF THE HEAD AND NECK}

The veins of the exterior of the head are as follows:

Facial.

Internal maxillary.

Temporal.
Temporomaxillary.

Posterior auricular.

Occipital.

(The student will bear in mind that the veins bear the same names as the arteries, and are generally in the same sheath; this is particularly true of the deep trunks.)

The facial vein takes its commencement on the anterior part of the head, from a plexus of the temporal branches. Under the name frontal vein it passes along the middle line of the forehead to the root of the nose, where it is connected with its fellow of the opposite side by a communicating branch. It is divided into several branches, which are distributed about the anterior part of the face; it receives, in its course, the supra-orbital, the frontal vein, and the dorsal veins of the nose; also all veins that correspond with the branches of the facial artery.

The internal maxillary vein joins the temporal vein.

The temporal vein drains the region of the temple and enters the parotid gland, after which it unites with the internal maxillary vein to form the temporomaxillary.

The temporomaxillary vein is formed by the union of the temporal and internal maxillary. It divides into two branches, one joining the facial vein, and the other joining with the posterior auricular to form the external jugular. 
The posterior auricular vein begins at the vertex, runs behind the ear, and helps to form the external jugular vein.

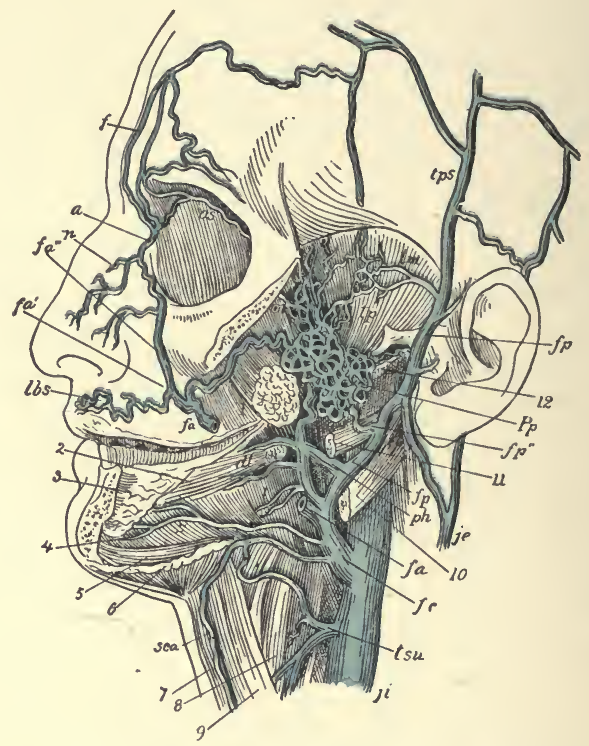

FIG. 6I.- The internal jugular and common facial veins and their branches. The zygoma and the left side of the inferior maxilla, together with the masseter, have been removed: I, Pterygoid muscle; 2 , styloglossus muscle; 3 , sublingual gland; 4 , hyoglossus muscle; 5 , geniohyoid muscle ; 6, mylohyoid muscle; 7 , sternohyoid muscle; 8 , thyrohyoid muscle; 9 , omohyoid muscle; IO, stylohyoid muscle; II, styloglossus muscle; I2, stylopharyngeus muscle; $j i, j e$, internal and external jugular vein; $p h$, pharyngeal vein; $d l$, dorsal vein of tongue; $l$, lingual veins; $s$, sublingual vein; $t s u$, superior thyroid vein; $f c$, common facial vein; $f a$, anterior facial vein ; $f a^{\prime}$, superficial, and $f a^{\prime \prime}$, deep branches of same; $f$, frontal vein; $a$, angular vein ; $o s$, superior ophthalmic vein ; $n$, nasal vein ; $l b s$, superior labial vein; $f p$, posterior facial vein ; $f p^{\prime}, f p^{\prime \prime}$, superficial and deep branches of same; tps, tpm, tpr, superficial, middle, and deep temporal veins; $P p$, pterygoid plexus; $o i$, inferior ophthalmic vein; $s c a$, anterior jugular vein (after Henle).

The occipital vein follows the direction of the occipital artery, terminating usually in the internal jugular.

The veins of the neck, which return the blood from 
the head and face, are the internal jugular, anterior jugular, external jugular, and vertebral.

The internal jugular vein is formed by the lateral and inferior petrosal sinuses outside of the jugular foramen; it unites with the subclavian to form the innominate vein. The facial, lingual, pharyngeal, superior, and middle thyroid veins unite to make up the supply on its course. At the base of the neck, where it unites with the subclavian vein, is a pair of valves.

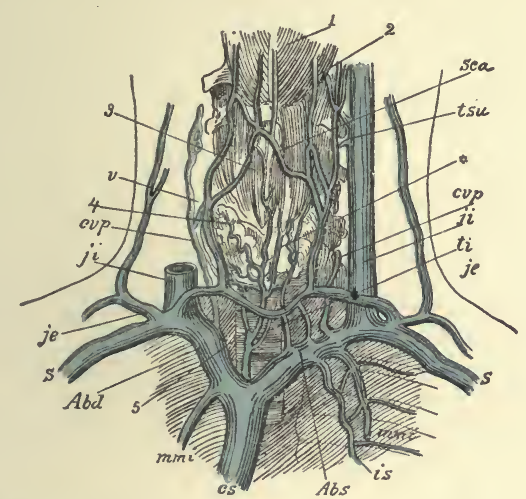

FIG, 62.-Innominate vein and its branches: I, Mylohyoid muscle; 2, root of tongue; 3 , larynx; 4 , thyroid gland; 5 , trachea; $c s$, superior vena cava; $A b d, A b s$, right and left innominate veins; $S$, subclavian vein ; $\ddot{j}, j e$, internal and external jugular veins; $t s u$, $t i$, superior and inferior thyroid vein; $c v p$, deep cervical vein; $v$, vertebral vein ; $m m i$, internal mammary ; is, superior intercostal vein; sca, anterior jugular vein; $*$ thyroid plexus (after Henle).

The anterior jugular begins by many small veins about the hyoid bone in the median line, and enters the subclavian vein.

The external jugular vein, formed by a division of the temporomaxillary and the posterior auricular, passes over the sternomastoid muscle, thence downward, and terminates in the subclavian vein.

The vertebral vein follows the course of the artery, 
but drains only the scalp in the occipital region, since it does not come from the cranial cavity. It enters the subclavian vein.

The inferior thyroid veins receive the blood from the thyroid gland. Each unites with its fellow, and, along with the middle and superior, they form a plexus in front of the trachea. The right terminates in the right innominate vein, and the left in the left innominate vein.

\section{VEINS OF THE UPPER EXTREMITIES}

The veins of the upper extremities are superficial and deep; the latter accompany the arteries, and are called vence comites; they are sometimes enclosed in the same sheath. The brachial vence comites are situated on each side of the artery, and unite with the basilic vein to form the axillary vein. The axillary becomes the subclavian, and the subclavian unites with the internal jugular to form the vena innominata.

The superficial veins of the arm are:

Anterior ulnar.

Posterior ulnar.

Basilic.

Radial.
Cephalic.

Median.

Median basilic.

Median cephalic.

The anterior ulnar vein collects the blood from the inner part of the hand, ascends to the elbow, and unites with the median basilic to form the basilic vein.

The posterior ulnar vein takes its beginning at the back of the hand, unites with the veins of the fingers, ascends to the back part of the elbow, and terminates in the anterior ulnar vein.

The basilic vein arises from the ulnar, and is formed by the coalescence of the median basilic with the ulnar veins; it is situated on the inner side of the arm, passing upward to the axilla, terminating in the axillary. 
The radial vein starts at the back of the wrist, receiving tributaries from the deep veins of the palm, and connects with the several venous branches from the fingers and thumb, where it is increased in size; it passes on upward, and at the bend of the elbow it runs forward and unites with the median cephalic to form the cephalic vein.

The cephalic vein passes upward on the outer side of the arm and terminates in the axillary vein.

$\because$ The median vein takes its start at the wrist and palm of the hand; at the elbow it receives other branches from the deep structures, and divides into two branchesthe median basilic and median cephalic.

The median cephalic unites with the radial to form the cephalic vein.

The median basilic unites with the ulnar to form the basilic vein.

The deep veins of the upper extremity follow the course

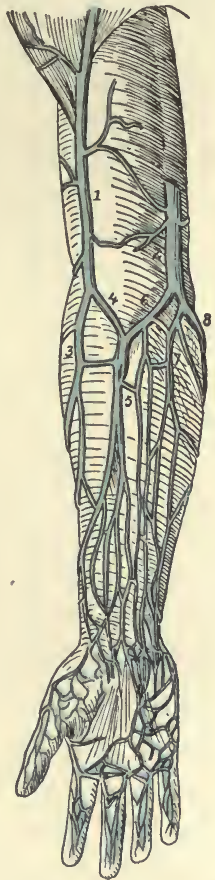

FIG. 63.-Superficial veins of upper limb: I, Cephalic vein; 2, basilic vein ; 3 , radial vein ; 4, median cephalic vein ; 5 , median vein; 6 , median basilic vein ; 7 , anterior ulnar vein; 8, posterior ulnar vein (after Quain). of the arteries. They have numerous anastomoses, not only with one another, but with the superficial veins.

The axillary/vein is formed by the union of the brachial venæ comites with the basilic; it also receives 
several other branches from the axillary region, and terminates in the subclavian.

The subclavian vein crosses the first rib and passes below the clavicle; it unites with the internal jugular

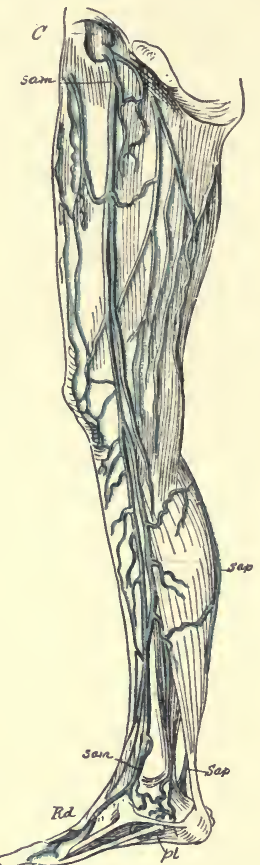

FIG. 64.-Superficial veins of lower limb: $C$, Femoral vein; Sam, Sap, long and short săphenous veins; $R d$, dorsal rete of foot; $p l$, plantar vein (after Henle). vein to form the innominate vein.

VEINS OF THE LOWER EXTREMITIES

The deep veins of the leg accompany the arteries in pairs, and form the venæ comites of the anterior and posterior tibial arteries. The tibial veins unite and form the popliteal vein.

The popliteal vein passes through the popliteal space and to the outer side through an opening in the adductor magnus muscle, and becomes the femoral vein. It usually contains four valves, and it also receives blood from the short saphenous vein.

The femoral vein commences at sthe opening in the adductor magnus muscle, and is enclosed in the femoral sheath; it enters the pelvis beneath Poupart's ligament, and then becomes the external iliac vein. The valves are about five in number. It receives several veins from the muscular structure, as well as the deep femoral and the long saphenous veins.

The deep femoral is made up of small branches that 
are associated with the artery of the same vein, and terminates in the femoral vein, below Poupart's ligament.

The short saphenous vein begins in the arch on the dorsum of the foot; it ascends the outer and back part of the leg, receives many branches from the posterior part of the leg, and perforates the deep fascia to terminate in the popliteal vein.

The long saphenous vein begins at the inner end of the arch on the dorsum of the foot, and passes to the inner part of the ankle; it then ascends to the knee, passes behind the condyle of the femur upward to the saphenous opening, where it enters the femoral sheath and terminates in the femoral vein below Poupart's ligament. It receives the cutaneous veins of the thigh, and unites with many deep branches. At the saphenous opening it unites with the superficial epigastric and superficial circumflex iliac veins, as well as with the superficial external pudic. This vein is of importance, for in phlebitis of the lower extremity it is generally implicated; in the varicosity which occurs in the leg it is also this venous trunk that is usually involved.

\section{VEINS OF THE TRUNK}

The innominate veins are two large trunks, formed one on each side, by the union of the internal jugular and subclavian. The right innominate unites with that of the opposite side and forms the superior vena cava; it receives the right internal mammary and the right inferior thyroid veins. The left innominate passes obliquely across the chest, and unites with the right innominate to form the superior vena cava.

The superior vena cava is a short branch, about 3 inches in length, formed by the union of the two innominate veins; it enters the pericardium and terminates in the right auricle of the heart. 
The external iliac accompanies the artery of the same name, and unites with the internal iliac to form the common iliac vein.

The internal iliac drains that area of the pelvis which is supplied by the artery of the same name, and unites with the external iliac to form the common iliac vein.

The uterine plexuses are situated about the vagina and uterus, between the two layers of the broad ligaments. The vesical and prostatic plexuses receive blood from the genital organs and the bladder.

Each common iliac vein is formed by the union of the external and internal iliac veins of the corresponding side; at the origin of the right common iliac artery the two common iliac veins unite and form the inferior vena cava.

The inferior vena cava, as has been said, is formed by the union of the two common iliac veins; it ascends along the vertebral column to the right of the abdominal aorta, passing through the diaphragm, and terminating in the inferior and posterior part of the right auricle of the heart; this vein contains no valves. It receives, in its course, the following veins: Lumbar, right spermatic, renal, suprarenal, phrenic, and hepatic.

The Azygos Veins.-These connect the superior and the inferior vena cava, taking the place of these vessels in that part of the chest occupied by the heart.

The superior intercostal veins receive the veins of the first and second intercostal spaces; the right terminates in the vena azygos major, the left in the left innominate. The vena azygos major drains the remaining intercostal spaces on the right side and empties into the superior vena cava. The remaining intercostal spaces on the left are drained by the left upper and lower azygos veins; both of these vessels frequently terminate in the vena azygos major. 


\section{The Vertebral and Spinal Veins.-The plexuses} of veins of the vertebral column and spinal cord are: The dorsispinal, meningorachidian, and medullispinal.

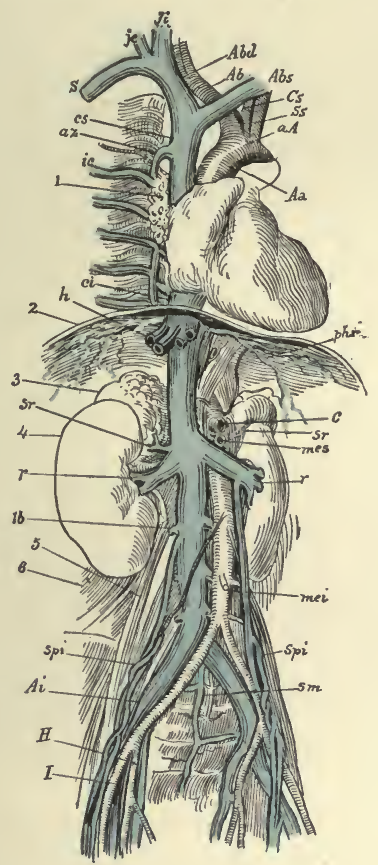

FIG. 65.-Veins and arteries of the thoracic and abdominal cavities: I, Root of right lung ; 2 , section of diaphragm ; 3 , suprarenal body ; 4 , kidney ; 5, psoas magnus muscle; 6 , quadratus lumborum muscle; $A a$, descending aorta; $a A$, arch of aorta; $A b$, innominate artery; $c s$, left carotid artery ; $S s$, left subclavian artery; $c$, celiac artery; mes, mei, superior and inferior mesenteric arteries; $c s, c i$, superior and inferior venæ cavæ; $A b d, A b s$, right and left innominate veins; $S$, subclavian vein $; j e, j i$, external and internal jugular ; $a z$, vena azygos major; $i c$, intercostal vein ; $h$, hepatic veins ; $p h r$, phrenic vein and artery; $s r$, suprarenal vein; $r$, renal artery and vein; $l b$, lumbar vein; spi, internal spermatic artery and vein; $s m$, middle sacral artery and vein; $a i$, common iliac artery and vein; $H$, hypogastric artery and vein ; $I$, iliac artery and vein (after Henle).

The Cardiac Veins.-These veins return the blood from the muscular structure of the heart. They are 
divided as follows: Great cardiac vein, posterior cardiac vein, anterior cardiac vein, and venæ Thebesii. They collect the blood from the several portions of the heart, and terminate in the right auricle.

\section{THE PORTAL CIRCULATION}

The portal circulation is a part of the general systemic circulation. The stomach, intestines, pancreas, and spleen receive their arteries from the aorta, and the blood, on leaving these, is received into various veins, which unite to form the portal vein; this enters the substance of the liver and breaks up into numerous capillaries. The portal vein not only returns venous blood from the gastro-intestinal tract, but also carries nutritive products to the liver, which are there still further elaborated. The blood is then collected by the hepatic vein, which opens into the inferior vena cava just before the vessel enters the right auricle.

The portal system of veins is formed by the union of the superior and inferior mesenteric, splenic, and gastric veins, and collects the blood from the digestive viscera. The resulting trunk divides in its course to the transverse fissure of the liver into a branch each for the right and left lobe, which ramify to form plexuses in the liver. The blood from the hepatic artery furnishes blood to this vein in the interior of the liver, and exterior to this organ the vein unites with the pyloric and coronary veins. The blood so collected passes through the liver and, as previously stated, is transmitted to the inferior vena cava by the hepatic vein.

\section{THE PULMONARY CIRCULATION}

The pulmonary artery, carrying venous blood, leaves the right ventricle and divides into branches, one for each lung. Inside the lung this vessel breaks 
up into small branches, which form a network around the alveoli, or air-cells. The oxygenated blood is collected by the pulmonary veins and carried to the left auricle.

The two auricles contract and force the blood into the ventricles; the two ventricles then contract, and the tricuspid and mitral valves close their orifices, the blood being propelled on into the large vessels (the pulmonary artery and the aorta), the semilunar valves then closing. The blood cannot get from the right side of the heart to the left without passing through the lungs, or lesser circulation; neither can the blood get from the left side of the heart to the right without passing around the general or systemic circulation.

The Pulmonary Veins.-The pulmonary veins are formed by the union of the capillaries in the lung; they unite in two trunks from each lung, discharging their blood into the left auricle of the heart. As has been stated before, this is an instance where the veins transmit arterial blood. In some instances there are three veins on the right side, which terminate in a common opening in the auricle. There are no valves in the pulmonary veins.

\section{THE BLOOD}

We have previously described the manner in which the blood passes through the heart in circulating through the body. It now remains for us to consider the function of the blood, and to give a description of the manner in which it is oxygenated and replenished with nutritive matter.

The blood is a homogeneous liquid, possessing a slight degree of viscidity, with a consistency and density somewhat greater than water. It is especially distinguished by its color, which is usually of a bright scarlet when it is drawn from an artery, and of a dark red or purple, some- 
times almost approaching to black, when it is drawn from a vein. It has a salty taste and a peculiar odor. The reaction is faintly alkaline; at a temperature of $60^{\circ} \mathrm{F}$. the specific gravity is I.055. The temperature of the blood as it passes through its course in the arteries is about $100^{\circ} \mathrm{F}$., but it may vary under different circumstances in health from fatigue, overaction, and other conditions. It coagulates rapidly, the serum separating and leaving the clot, which is termed the crassamentum.

Distribution.-After leaving the arteries the blood passes through the capillary networks of the various tissues, where the nutriment diffuses through the delicate vessel walls and nourishes the part. At the same time the waste materials resulting from tissue changes are thrown into the circulation to be carried away and eliminated from the body.

The blood is composed of the plasma or liquor sanguinis, and of the corpuscles. The plasma contain proteids, fats, extractive matters, and salts.

The Corpuscles.-The corpuscles of the blood are of two kinds, and are known as the red and the white corpuscles. The red corpuscles exist in greater number than the white. The blood, therefore, is a fluid, which provides a means of suspending these important constituents; this fluid is named liquor sanguinis or "plasma," and must not be confused with the "serum" (see p. 136), which is formed during the coagulation of blood. It is serum and something more, for it contains one at least of the elements or factors from which fibrin is formed.

The table from "Gray's Anatomy" will show the relation, which will be easily understood by the nurse:

$$
\text { Blood }\left\{\begin{array}{l}
\text { Corpuscle }\left\{\begin{array}{l}
\text { Colored } \\
\text { Colorless }
\end{array}\right. \\
\text { Liquor sanguinis }\left\{\begin{array}{l}
\text { Fibrin } \\
\text { Serum }
\end{array}\right.
\end{array}\right\} \text { Clot. }
$$


The relative proportion of the white to the red corpuscles has been variously estimated by many observers, and varies from I to 400 to I to 1200.

The red corpuscles (erythrocytes) are round biconcave discs; their average diameter is estimated to be about $\frac{1}{3200}$ of an inch. They vary in size when disease, such as anemia, exists.

Composition.-The composition of the red cell is estimated to be as follows:

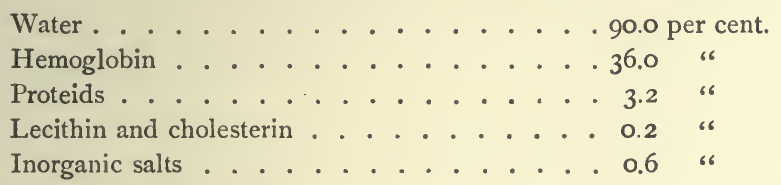

Their color is due to the hemoglobin which they contain; by some the color is said to be due to iron. The function of the red corpuscles is to carry oxygen (through the intervention of the hemoglobin they contain); therefore, they are denominated "oxygen carriers." The blood

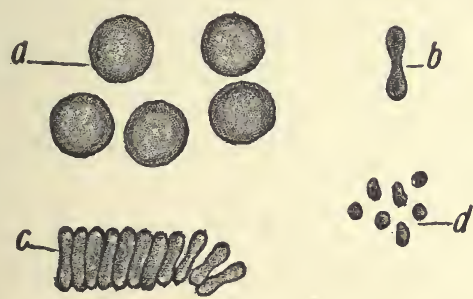

FIG. 66.-Cells of blood: $a$, Colored blood-corpuscles seen on the flat; $b$, on edge; $c$, in rouleau; $d$, blood-platelets (Leroy).

passing to the lungs receives a new supply of oxygen, and returns to the tissues, where combustion takes place, which deposits the oxygen, whereby heat and energy are produced; it now takes on new substances (carbon dioxid) 
and returns to the lungs (changed in character from red to blue blood) through the venous system.

The red corpuscles have a remarkable power of absorbing certain gases; for it has been found by Scherer that a solution of hematin imbibes a considerable amount of oxygen, which is the principal constituent of the composed strength of the corpuscle itself.

When examined beneath the microscope, the red corpuscles may be seen floating in the liquor sanguinis of the blood, exhibiting a marked tendency to approximate one another; usually coming into contact by their flattened surfaces, so that a number of them aggregated present the appearance of a pile of coins set upon their edges (rouleaux). If the stratum should be too thin to permit them to lie in this manner they will overlap one another, especially so when the current is impeded. They will be seen at times to change their shape, become elongated, yet afterward resuming their natural shape; the elongation is due to pressure from without, or from the diminution of the interior of the vessel in which they are contained.

The white corpuscles are called lenkocytes; they are few in number in comparison with the red; they are spheroid in form, and measure about $\frac{1}{2500}$ of an inch in diameter. The average proportion of the white to the red is I to 500 ; in certain diseased conditions the white cells are rapidly increased; this abnormal state is called leukocytosis.

The white corpuscle has no limiting membrane like the red corpuscle; it consists of a mass of transparent albuminous substance called protoplasm, containing one or more nuclei. They are endowed with the power of spontaneous movement, and are thus capable of changing their'form and place. 'The nuclei vary in shape: they may be crescentic, horseshoe shaped, or multiple.

In their movements they pass through the vessels into 
the lymph-spaces. In inflammations the cells pass out in great numbers, and in suppurative conditions they become pus cells.

The white cells migrate to the part infected, surround the substances, and, through their protective powers, prevent further intoxication of the system. This surrounding and absorption of infective matter is termed phagocytosis, and the white corpuscles, when acting in this manner, are spoken of as phagocytes.

This phagocytic action is aided by a certain chemic substance, called opsonin, which exists in the blood-plasma,

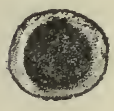

a

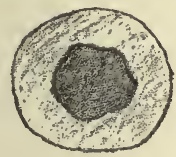

6

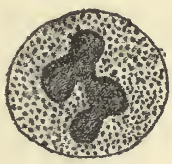

c

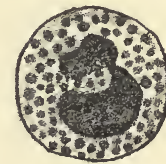

$\alpha$

FIG. 67.-Various forms of leukocytes: $a$, Small lymphocyte; $b$, large lymphocyte; $c$. polymorphonuclear neutrophile; $d$, eosinophile (Leroy).

and whose function is to act on disease germs, so as to prepare them for absorption by the white cells.

The blood-plates are small colorless or yellowish bodies of varying shape, but considerably smaller than the red corpuscles. It is still uncertain whether these bodies are independent structures or only fragments of destroyed red corpuscles.

The plasma of the blood is a white, yellowish, clear fluid, largely composed of water, holding in solution various salts, proteid substances, fats, etc. The proteid materials are made up of albumin, paraglobulin, and fibrinogen; the first two occur in about equal quantities, while the last is found in small quantities.

Nutrition is gained through the albuminoids, which replenish the tissues of waste materials taken up through the processes of combustion and disease. 
The paraglobulin has about the same effect as albumin. The fibrinogen produces coagulation; its power to coagulate is such that it is impossible to obtain it in a fluid condition.

The mineral salts maintain the alkalinity of the blood. The salt which occurs in largest quantity is sodium chlorid.

Gases.-Besides the constituents just mentioned, the blood contains oxygen and carbon dioxid; Ioo volumes of the blood contains about 50 volumes of these gases collectively. The proportion varies considerably, but arterial blood always contains about twice as much oxygen and a third less of carbon dioxid than venous blood. The several constituents of the serum vary with age, sex, and temperament. The quantity of water is greater in females than in males; in children and aged persons than in persons of middle life, and in the lymphatic temperament than in the sanguine. In healthy males it has a range of from 760 to 800 parts in 1000, and an average of 779 ; in healthy females a range of 773 to 813 , with an average of $79 \mathrm{I}$.

The quantity of blood contained in the human body is said to be about $\frac{1}{13}$ of the body weight.

Clotting of the Blood.-When the blood is drawn from a person in health, it solidifies in a very short time into a jelly-like mass, but retains its color and volume. After it has remained a longer time there will be noticed a separation of the constituents, with the formation of a central substance which, after a time, begins to become smaller. After ten to twenty hours what was formerly a coagulated mass of blood becomes separated into two parts, a clot (crassamentum) and a considerable quantity of yellowish fluid, which is known as serum, in which the clot is suspended. The clot thus formed consists of fibrin, which is associated with a large number of cells or bloodcorpuscles. Blood, while in the process of solidification, 
undergoes a chemic action with the air through the medium of the blood ferment, fibrinogen, which glutinates the corpuscles with the other constituents to form the clot. This ferment does not exist as such in the blood when contained in the blood-vessels, but is supposed to be the result of the destruction of the white corpuscles and the blood platelets. Some authors assume that these platelets contain a nuclein body, coupled with proteids, which is the essential factor in the coagulation of the blood. It is thought that these structures disintegrate very rapidly when blood is drawn from the body, liberating the ferment, and so producing coagulation.

Changes in Blood in Disease.-The blood undergoes various changes in disease. Traces of organic compounds, viz., uric acid, urea, keratin, keratinin, taurin, cholesterin, and leucin, may also occur in the blood, and cause serious, if not fatal, derangement.

There may occur variations in the relative proportion of its constituents; for instance, in plethora the red corpuscles are said to be increased in number, while in anemia they are deficient in number or quantity. After the loss of great numbers of the red blood-corpuscles they are slowly reproduced, hence the long-continued pallor after hemorrhages. The fibrin is increased in acute inflammation, especially in those of the serous membranes, in acute rheumatism, pneumonia, erysipelas, phthisis, tonsillitis, and inflammation of the lymphatic vessels. It is always in excess in the pregnant female. On the other hand, it is always diminished in enteric fever, cerebral hemorrhage and congestion, in scurvy, and after profuse hemorrhages. The albumen increases in Bright's disease and glandular inflammation, erysipelas, scarlet fever, and tuberculosis. The salts of the serum are diminished in enteric fever and cholera. Mixture with foreign substances from the inhalation of noxious gases may occur, or the 
respiration may become obstructed and the blood become overcharged with the poison. When the liver is inflamed or its ducts obstructed, the constituents of the bile are retained in the blood or pass into it by transudation. In acute nephritis urea is, in like manner, retained within the system and induces the gravest symptoms.

Of the morbid products which find their way into the blood, one of the most harmful is the germ of suppuration, which, under certain circumstances, is absorbed from the veins or from suppurating surfaces. Extraneous matters such as toxins, germs, or both, may be introduced by the stomach, skin, and lungs, or more directly by penetrating wounds.

The quantity of blood is increased by food and in plethora. It is diminished in hemorrhage and abstinence. It is also said to be diminished in anemia; but the pallor which characterizes that disease is due to a deficiency in the number of the red blood-corpuscles, as well as to the altered constituents of the same. The temperature of the body is increased in diseases accompanied with an increased circulation, as in severe inflammatory fevers. On the other hand, it is lowered in languid states of the circulation, as in apnea, and in poisoning by some drugs.

\section{THE VASCULAR SYSTEM OF THE FETUS}

The arterial blood destined for the nutrition of the fetus is carried from the placenta to the fetus, along the umbilical cord, by the umbilical vein. The umbilical vein enters the abdomen at the umbilicus, and passes upward along the free margin of the suspensory ligament of the liver to the under surface of this organ, where it gives off two or three branches to the left lobe; one of these is of large size; other branches are given off to the several other lobes of the liver. At the transverse fissure of the 
liver the umbilical vein divides into two branches; of these, the larger joins the portal vein and enters the right lobe;

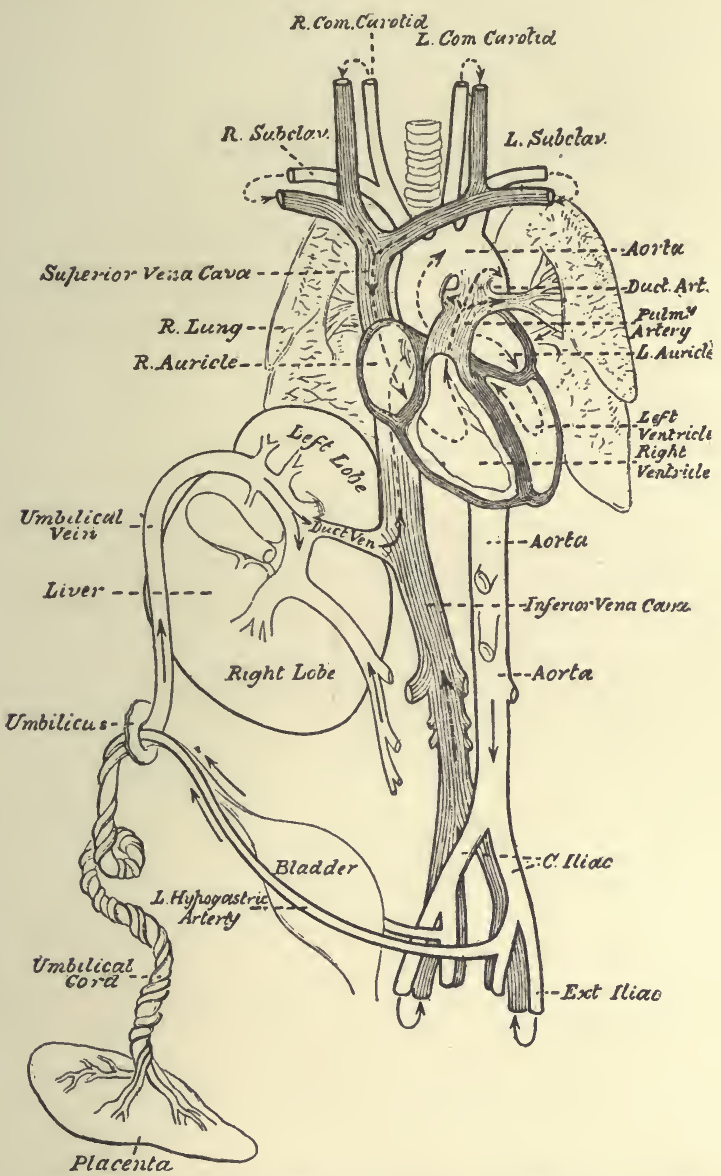

FIG. 68.-Diagrammatic view of the fetal circulation (Dorland).

the smaller branch continues onward, under the name of the ductus venosus, and joins the left hepatic vein at the point 
of junction of this vessel with the inferior vena cava. The blood, therefore, that traverses the umbilical vein reaches the inferior vena cava in three different ways: The greater quantity circulates through the liver with the portal venous blood before entering the vena cava by the hepatic veins; some blood enters the liver directly, and is also returned to the inferior vena cava by the hepatic veins; the smaller quantity passes directly into the vena cava by the junction of the ductus venosus with the left hepatic vein.

In the inferior vena cava the blood carried by the ductus venosus and hepatic veins becomes mixed with that returning from the lower extremities and viscera of the abdomen. It enters the right auricle, and, guided by the Eustachian valve, passes through the foramen ovale into the left auricle, where it becomes mixed with a small quantity of blood returned from the lungs by the pulmonary veins. From the left auricle it passes into the left ventricle, and from the left ventricle into the aorta, whence it is distributed almost entirely to the head and upper extremities, a small quantity being carried into the descending aorta. From the head and upper extremities the blood is returned by the branches of the superior vena cava to the right auricle, where it becomes mixed with a small portion of the blood from the inferior vena cava.

From the right auricle it descends over the Eustachian valve into the right ventricle, and from the right ventricle it passes into the pulmonary artery. The lungs of the fetus are solid and almost impervious, hence only a small quantity of blood is distributed to them by the right

- and left pulmonary arteries, and this is returned by the pulmonary veins to the left auricle; the greater part passes through the ductus arteriosus into the commencement of the descending aorta, where it becomes mixed with a small 
quantity of blood transmitted by the left ventricle into the aorta. Along this vessel it descends to supply the lower extremities and viscera of the abdomen and pelvis, the chief portion being, however, conveyed by the umbilical arteries to the placenta.

The placenta serves the double purpose of a respiratory and nutritive organ, receiving the venous blood from the fetus, and returning it again reoxygenated and charged with additional nutritive material.

Changes at Birth.-At birth, when respiration is established, an increased amount of blood from the pulmonary artery passes through the lungs, which now perform their office as respiratory organs, and, simultaneously, the placental circulation is cut off. The opening between the two auricles (foramen ovale) now becomes gradually closed in, generally about the tenth day; valvular folds now form about the valvular opening or foramen; sometimes this opening is persistent and never closes.

The other parts of the ducts that furnish the circulation from the after-birth, through the medium of the funis, or cord, become obliterated and form attachments to the several organs of the internal viscera.

The illustration (Fig. 68) will give a fair idea of the course of the blood to and from the after-birth, and of the location of the arterial structure.

The ductus arteriosus forms a cord that connects the left pulmonary artery to the concavity of the arch of the aorta.

The umbilical arteries form ligaments to the bladder, and the umbilical veins and ductus venosus become obliterated to form fibrous cords. The remains of the umbilical vein forms the round ligament of the liver. 


\section{REVIEW QUESTIONS}

What constitutes the circulatory system?

Give the circulation of the blood.

What is the heart, and where is it located?

How is the heart subdivided?

What membrane encloses the heart?

Name the valves contained within the heart, and location of each.

What valves present themselves at the beginning of the aorta?

What takes place when the ventricles contract?

How are the arteries distinguished?

In what direction does the arterial blood flow?

Give a description of the arteries.

What are the capillaries?

What is the aorta, and how is it divided?

What important arteries are given off from the abdominal aorta?

What are the coronary arteries? What do they supply?

What important arteries pass to the brain?

What arteries supply the face with blood?

What does the occipital artery assist in supplying?

What important structures does the temporal artery supply?

What artery pierces the dura mater and divides into three branches?

What does the ophthalmic artery supply?

What artery supplies nutrition to the eye?

Describe the subclavian artery.

What do you understand by the vertebral arteries?

What important artery do the vertebral arteries form?

What muscles does the internal mammary artery supply?

Name the artery that supplies the intercostal spaces and pleura.

What artery is in the axillary space?

Under what bone do we find the subclavian artery?

Of what is the radial artery a branch?

What artery lies on the outer side of the forearm?

Which is the larger, the ulnar or the radial artery?

What forms the palmar arch in the hand?

What is the abdominal aorta, and what important structures does this supply?

What artery supplies the diaphragm?

What artery supplies the liver?

What important structure does the splenic artery supply?

Into how many branches does the splenic artery divide?

What artery provides nutrition to the small intestine? 
What artery supplies the upper part of the duodenum and pancreas?What other name has the spermatic artery?

What important structures do the inferior mesenteric artery supply?

Name the artery which supplies the kidneys.

What artery provides nutrition for the pelvic organs?

What artery passes down the leg, and how is it divided?

What artery forms the plantar arch?

Give a description of the pulmonary artery.

What is the venous system?

What important veins have we in the neck?

In what direction does the blood flow in the veins?

What are sinuses, and where are they located?

What do veins contain?

What large vein in the lower extremity frequently becomes diseased?

What forms the superior vena cava? The Inferior?

What do the azygos veins connect?

What are plexuses, and where do we find them?

Through what veins does the blood from the substance of the heart return?

What makes up the portal circulation? Describe its location.

What do you understand by the pulmonary circulation?

What is the foramen ovale?

Where would you make pressure in a lacerated limb if an artery was severed?

Describe the red corpuscles; the white.

What is the function of the hemoglobin?

What is the function of the plasma?

What is the composition of the blood?

What is the average quantity of blood in the adult?

Through what medium does the fetus derive its nourishment?

What is the difference in the circulation of the fetus before birth and after? What takes place after birth?

What are the changes that take place in the blood?

On what is the pulse dependent? 


\section{H A P TER I V}

\section{THE RESPIRATORY SYSTEM}

THE substances furnished to the circulation from the alimentary canal are in a condition that, with reference to

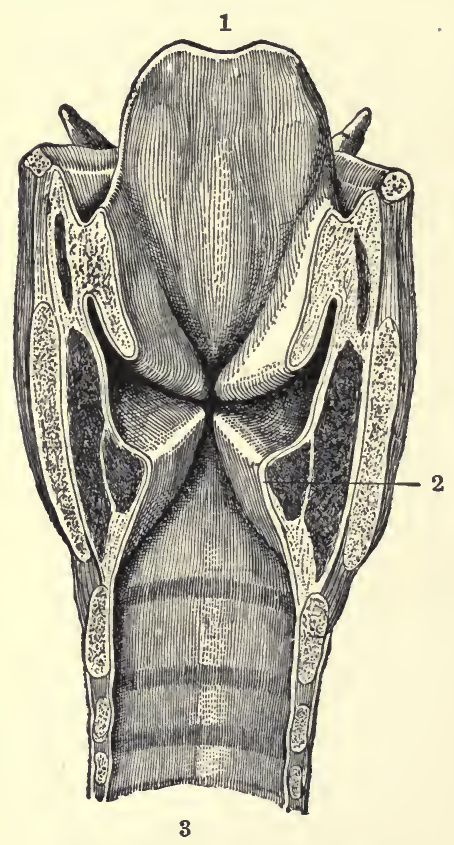

FIG. 69.-Interior of the larynx : I, Epiglottis; 2, vocal cord ; 3, cavity of the trachea (after Testut). the atmosphere, will admit of free combination with oxygen: this latter agent is quite necessary to prepare the tissues for the functions of assimilation and nutrition. Besides this, the venous blood is also charged with carbonated products that, when exposed to oxygen, are eliminated and passed off in the form of carbon dioxid. To effect these several purposes and thus to maintain the motive power of the system, eliminating, at the same time, deleterious substances, is the function of the apparatus of respiration.

The repiratory organs consist of the larynx, trachea, and lungs. The larynx is composed of cartilages, held together by ligaments, and contains the vocal cords. 
The trachea is a membranous tube with cartilaginous rings, which, upon its entrance into the chest, divides into right and left bronchi. It is about 5 inches in length and $\frac{3}{4}$ inch in diameter.

The lungs are so constructed as to receive a very large amount of atmospheric air by the most extraordinary mechanism. The entire extent of the respiratory surface in the lungs has been estimated at roo times that of the cutaneous surface of the body; the blood in the pulmonary capillaries, distributed in this large surface, and being in immediate proximity to the air in the cavity of the vesicles, is placed under the most favorable conditions for its rapid and complete oxygenation.

Each pulmonary vesicle is covered upon its exterior with a close network of capillary blood-vessels, which penetrate into the septa between it and the adjacent cavities, and which are thus exposed on both sides to the influence of the atmospheric air. The walls of the vesicles, as well as the interspaces between the lobules, are supplied with an abundance of elastic tissue, which gives to the pulmonary structure its property of resiliancy.

Thus the oxygen of the air combines with the blood, while a portion of the deleterious material also takes on new combinations and passes off with the carbon dioxid.

\section{THE LUNGS}

The lungs are situated in the uppermost portion of the chest, and, when inflated, completely fill that cavity. They are of irregular shape and two in number - one right and the other left. Each is enclosed in a sac formed by the pleural membrane; that portion of the thoracic cavity between the right and left pleural sac is known as the mediastinum. The right lung is the larger of the two, and is divided by two fissures into three lobes, whereas the left lung is divided by one fissure into two lobes. 
In their superior portion the lungs receive the trachea or windpipe, which divides into the bronchi, one of which goes to the right lung and the other to the left. These bronchial tubes continue to divide and branch off in the parenchymatous substance of the lungs until they become extremely minute in their ultimate attenuation. The lungs, like the heart, are conic in shape. Fig. 70 will

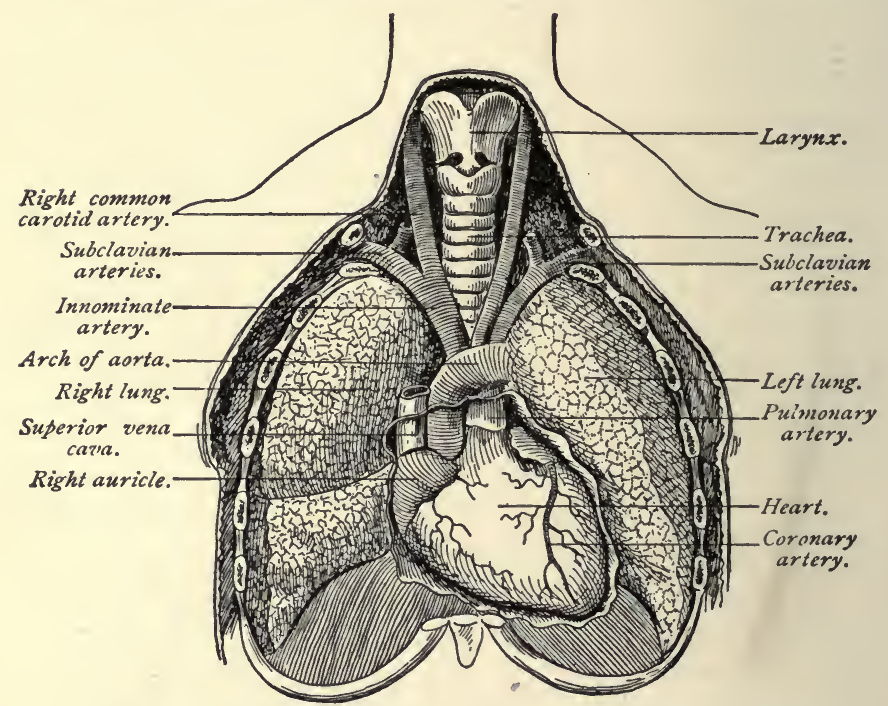

FIG. 70.- Relation of lungs to other thoracic organs (Ingals).

give some idea of the shape of the lungs and the position of the heart; it will also serve to illustrate the arrangement by which the blood and the air in the lungs become exposed by contact.

The substance of the lungs is of exceedingly light and spongy texture, being made up of air-cells, vessels, and tubes with their delicate walls. The color of the lungs varies with the age of the subject, being a light pinkishred in youth, and becoming darker, purplish, or mottled. 
in old age. The weight of the lungs is about 42 ounces, the right lung being 2 ounces heavier than the left.

The lungs, like the other organs, are supplied with nerves and vessels for their nutrition and support.

Respiration.-The respiration in health is free, easy, and noiseless. The number of respirations a minute

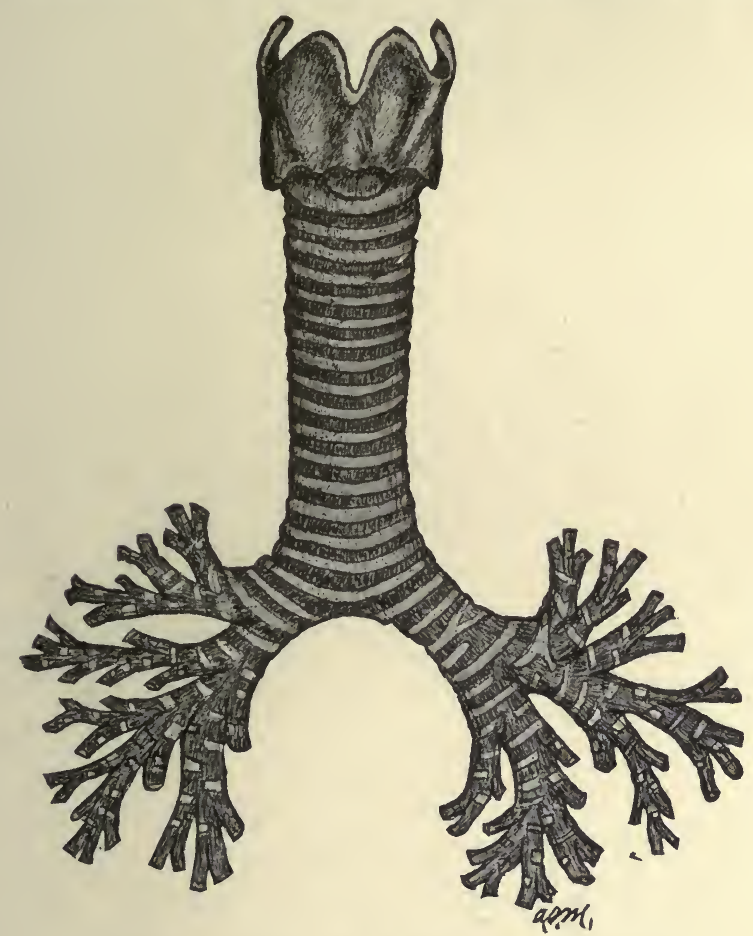

FIG. 71.-Larynx, trachea, and bronchi (Morrow).

varies, averaging about 35 during the first year of life, 25 during the second, 20 at puberty, and 18 in the adult.

The air which is contained in the pulmonary lobules and vesicles, being used for the purpose of arterializing the blood, becomes rapidly vitiated in the process of respira- 
tion, and requires accordingly to be as rapidly expelled and replaced by a fresh supply. The exchange or renovation of the air is effected by alternate movements of the chest of expansion and collapse, which follow each other in regular succession, and which are known as the movements of inspiration and expiration.

In inspiration the lungs fill with air, in expiration the air is expelled. The movements of respiration are involuntary in character, and even their acceleration or diminution is regulated by influences beyond our control. We may for a short time control these movements, but that for a limited time only, as the nervous impulse be-

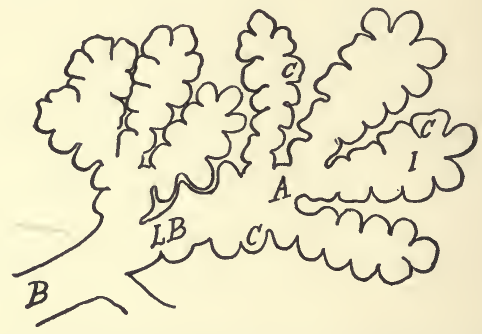

FIG. 72.-Diagrammatic representation of the termination of a bronchial tube in a group of infundibula: $B$, Bronchial tube; $L B$, bronchiole: $A$, atrium ; $I$, infundibulum; $C$, alveoli (Nancrede).

comes so very active that we are forced to breathe. Then again, if we try to breathe more rapidly than the normal respirations, we become fatigued and return to the normal standard. Respiration is, therefore, automatic in character, as may be noticed in persons during sleep; it is like the heart in that it requires no effort upon our part to maintain its action.

The respiratory movements vary according to age in sleeping and exercise. There are four pulsations of the heart to one respiratory act.

The air which is drawn into the lungs in respiration is 
a mixture of oxygen and nitrogen in the proportion of about 2I parts of oxygen to 79 parts of nitrogen. It also contains about .05 per cent. of carbon dioxid, a variable quantity of moisture, and some traces of ammonia.

If we examine the air at expiration, after passing through the lungs, we shall find that it has become altered in the following particulars: First, it has lost part of its oxygen; second, it has gained carbon dioxid, moisture, and organic matter.

The amount of carbon dioxid given off in the expired air, or the amount of oxygen that it contains, is dependent upon the gross amount of carbohydrates that have been consumed by the individual. Tigerstedt, in his description of this important action, says: "The percentage of oxygen in the expired air is, of course, less than that of the inspired air, and, in fact, it decreases more, as a rule, than the per cent. of $\mathrm{CO}_{2}$ (carbon dioxid) increases." When carbon burns in oxygen, the volume of the gas does not change. Since in respiration, however, the amount of oxygen which has disappeared is greater than that of the carbon dioxid formed, it follows that the oxygen is used in the body for other oxidations than that of carbon. The ratio between carbon dioxid formed and oxygen used, $\frac{\mathrm{CO}_{2}}{\mathrm{O}_{2}}$, is called a respiratory quotient.

The value of the respiratory quotient is very different under different circumstances, and depends upon the kind of food-stuffs which at the time are being burned in the body. The carbohydrates contain in their molecule just as much oxygen as is necessary to completely utilize their hydrogen. The total quantity of the inspired oxygen, therefore, can be used for oxidation of their carbon. The nurse's attention is directed to the following classification of the volumes of air at rest and in motion during 
pulmonary ventilation: (I) The residual air, or that part that cannot be expelled from the lungs, but remains after a full and forcible expiration, averages I 20 cubic inches; (2) the supplemental or reserve air, or that which can be expelled by forcible expiration after an ordinary outbreathing, measures I30 cubic inches; (3) the breath, tidal or breathing air, averages 26 cubic inches; (4) the complementary air, or that which can be inhaled after an ordinary inspiration, measures Ioo cubic inches. Thus, this estimate gives 250 cubic inches as the average volume of air that the lungs contain after an ordinary inspiration.

From what has been said it is apparent that the blood is constantly exposed to the air in the lungs; and it would appear that the residual air, or that which remains in the lungs after the expiration, is most exposed to the affinities in the materials of the blood, and, in all probability, it is upon this portion that the greatest impression is made. The inspiration replaces this residual air by admixture with the fresh portions inspired, and some of the expirations remove the mixed air so formed.

Many experiments have been tried to determine the amount of change of air in the lungs produced by a single breath. From the nature of the problem no method is wholly satisfactory. The one originally proposed by "Davy" is given by Prof. N. Kuntz, of Leipzig. It consists of inhaling a known volume of pure hydrogen, and then analyzing the expired air. If no mixing of the hydrogen with the air already in the lungs takes place, then the expired gas, like the inspired, would be pure hydrogen. By actual experiment it is found that, supposing that 500 c.c. of pure hydrogen are inhaled, only 170 c.c. of the hydrogen are exhaled, the remaining 330 c.c. being air. That is, 330 c.c. of the inhaled hydrogen displaces the same amount of air. If, now, it is assumed that in ordinary breathing 500 c.c. of air are inhaled, and 330 c.c. 
of this remain as with the hydrogen, and displaces a similar amount of vitiated air, then the amount of renewal must be the ratio between the air in the lungs before the inspiration - that is, the reserve and the residual air ( 3200 c.c.), and the fresh air (33o c.c.) remaining in the lungs after each respiration $\left(\frac{330}{3200}\right)=0.103$. This has been termed the coefficient of ventilation, and the case given shows that only about one-tenth of the air in the lungs is changed at each breath. The intermixture of this fresh air in the air-passages is due largely to the currents produced by inspiration, but the residual air in the alveoli and smallest air-passages must depend mostly on the jars produced by the heart-beats and the pulsation of the pulmonary arterioles, and also on the ciliary currents in the bronchi, facilitated by the swaying to and fro of the air as the chest alternately expands and contracts.

It would seem reasonable to suppose, therefore, that the air in the alveoli remains of a nearly uniform percentage of carbon dioxid, and a less percentage of oxygen than the expired air. The direct experiments of Wolfberg and others, however, show there is but little difference in the composition of the alveolar air and that which may be expired by a full expiration. This seems to show that the diffusion in the lungs is very rapid.

The trachea and bronchi are kept in a distended state, and their cylindric form maintained by the elastic annular cartilages of which the walls of these tubes are constructed. When in the lungs these cartilages are less apparent, as the tubes ramify and diminish, until we find the walls consisting simply of an elastic but very delicate membrane.

The accessory organs of respiration are the diaphragm and the intercostal and abdominal muscles; in difficult respiration other muscles of the chest are called into play. These muscles are all under the control of the respiratory 
center. The principal nerves concerned in respiration are the phrenic and the pneumogastric or par vagum.

Each pleural cavity is a closed sac, one occupying the right, the other the left half of the thorax; they are perfectly separate and do not communicate. They meet in the middle line of the chest at the upper part only, just behind the upper part of the gladiolus of the sternum. Lower down a space (the mediastinum) is left between

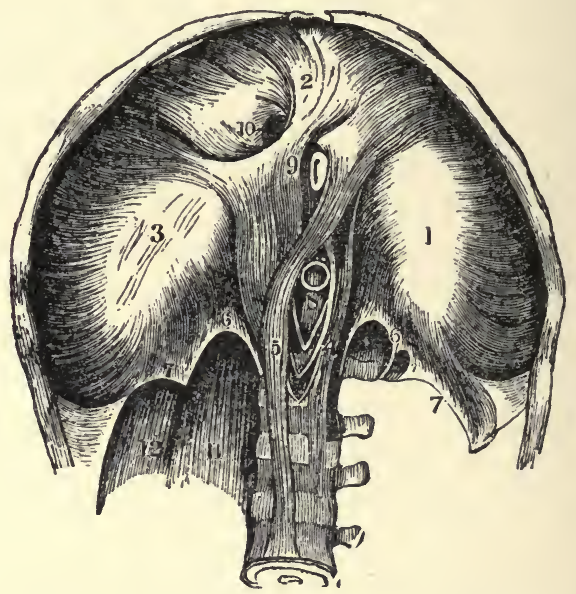

FIG. 73.-Interior view of the diaphragm: $1-3$, The three lobes of the central tendon, surrounded by the fleshy fasciculi derived from the inferior margin of the thorax; 4,5 , the crura; 6,7 , the arcuate ligaments; 8 , aortic orifice; 9, esophageal orifice; ro, quadrate foramen; II, psoas muscle; 12, quadrate lumbar muscle.

them, which contains all of the viscera of the thorax excepting the lungs.

The mediastinum is the space left in the median portion of the chest by the non-approximation of the two pleura; it extends from the sternum in front to the spine behind. It is divided into an upper and a lower portion: the upper is called the superior; the lower or inferior is divided into three subdivisions, called respectively the 
anterior, middle, and posterior mediastina, which have their several anatomic boundaries.

The ribs are elevated during inspiration and depressed during expiration; the chest is consequently expanded when the air is taken in and contracted when it is given out.

\section{THE DIAPHRAGM}

The diaphragm is a firm, musculofibrous septum, situated transversely across the cavity of the trunk, at the lower margin of the bony walls of the chest. It is convex on its upper, and concave on its lower, surface. By the contraction of the muscle-fibers in inspiration, the plane of the diaphragm becomes flattened and the lungs swell and fill the enlarged cavity of the chest, the air rushing in, on the principle of common air-pressure.

\section{REVIEW QUESTIONS}

What constitutes the respiratory system?

What is respiration?

Into how many acts is it divided?

What takes place in respiration?

How are the lungs divided?

What is the weight of the lungs?

Where are they situated?

What membrane surrounds them?

Which lung is the larger?

What is the trachea?

How do the bronchial tubes divide?

What are the volumes of air called?

State the average volume of air taken in at an ordinary inspiration.

Define reserve air, complementary air, and supplementary air.

What are the constituents of the inspired air, and also of the expired air?

What accessory organs are involved in respiration?

Describe the pleura.

What is the mediastinum?

Describe the diaphragm.

What nerve controls respiration? 


\section{H A P T E R V}

\section{THE DIGESTIVE SYSTEM.}

THE lungs, as we have seen in the chapter on the Respiratory System, supply oxygen to the blood and remove certain waste products. The tissues of the body, however, require many other substances besides oxygen to maintain life.

The needed nutrition is derived from the food we eat. The process by which this food is altered in character and prepared so as to become available and suitable for distribution to the different parts of the body is known as digestion.

Digestion is one of the most important functions performed in the human system; and any considerable deviation from its regular action has a ruinous influence upon the health. In consequence of the great number of organs concerned in digestion, the process is subject to frequent disturbances; these occur, more or less, in every disease to which the human frame is liable.

It is only by considering the great end of the digestive process that we can fully appreciate its vast importance to the animal economy. By this process our food and drink are prepared to yield their nutritious particles to the blood, from which all the other fluids, as well as the solids, are made, and upon which our very existence depends. Whenever, therefore, digestion becomes enfeebled, vitality must also become weakened; and a long-continued weakness of the digestive organs must produce disease, and ultimately death. 
The Alimentary Tract.-The digestion of food takes place in the series of organs known as the alimentary tract or canal. This begins at the mouth, and includes

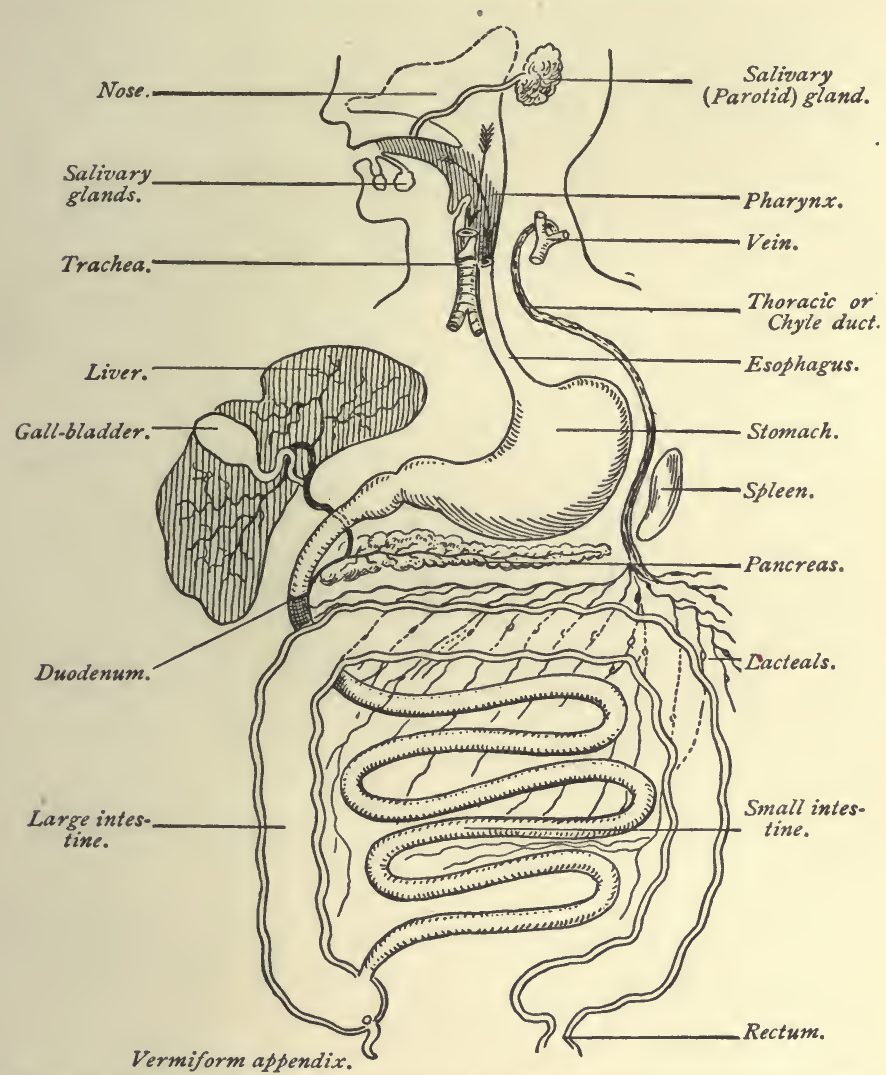

Fig. 74.-General scheme of the digestive tract, with the chief glands opening into it (Stockton).

the esophagus, stomach, and intestines, the organs chiefly concerned in the digestion of food. It is through this canal that aliment or food passes, until, having under- 
gone various changes, it yields its particles to the blood in the form of chyle.

Mastication.-By mastication is meant the trituration of food, a process accomplished by the teeth and the lower jaw, under the influence of muscular contraction.

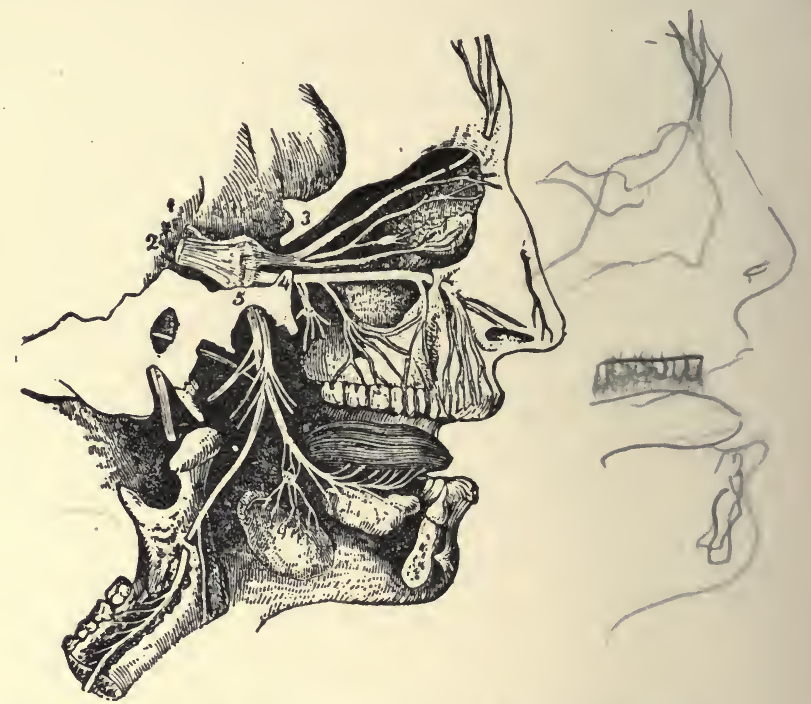

FIG. 75.-General plan of the branches of the fifth pair: I, Lesser root of the fifth pair; 2 , greater root, passing forward into the Gasserian ganglion; 3, placed on the bone above the ophthalmic division, which is seen dividing into the supra-orbital, lacrimal, and nasal branches, the latter connected with the ophthalmic ganglion; 4 , placed on the bone close to the foramen rotundum, marks the superior maxillary division; 5 , placed on the bone over the foramen ovale, marks the inferior maxillary division (after a sketch by Charles Bell).

At the same time the food becomes mixed with saliva and softened. When thoroughly divided, the food presents a greater surface for the solvent action of the digestive fluids, thus aiding the general process of digestion.

The lower jaw is capable of making a downward, an upward, a lateral, and an anteroposterior movement, 
dependent upon the construction of the temporomaxillary articulation.

The movements of mastication, though originating in an effort of the will and under its control, are, for the most part, of an automatic or reflex character, taking place through the medium of the medulla oblongata and induced by the presence of food within the mouth. The nerves and nerve-centers involved in this mechanism are the following:

Afferent, or Excitor Nerves: I. Lingual branch of the fifth pair. 2. Glossophary"ngeal.

Efferent, or Motor Nerves: I. Third branch of the fifth pair. 2. Second hypoglossal. 3. Facial.

The medulla oblongata not only generates motor impulses, but co-ordinates them in such a manner that movements of mastication may be directed toward the accomplishment of a definite purpose.

Insalivation.-Insalivation is the incorporation of the food with the saliva, secreted by the parotid, submaxillary, and sublingual glands. This process will be explained in the chapter devoted to the Glandular System.

Saliva.-Saliva is a colorless liquid, viscid in character, having a specific gravity of $\mathrm{I} .005$; its composition is:

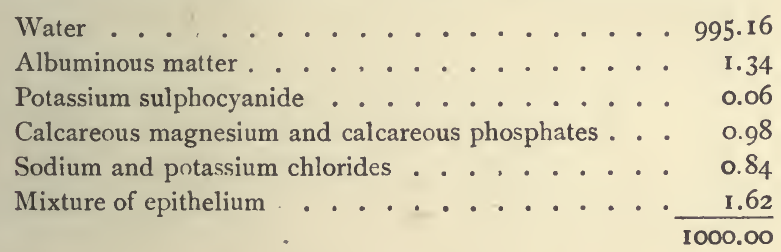

The viscidity of the saliva is due to the mucin which it contains. Saliva also contains an enzyme, called ptyalin, which acts upon the starchy particles of food to convert them into dextrin and sugar; and, finally, by the process of hydrolysis into dextrin and maltose. The 
amount of saliva secreted in twenty-four hours has been estimated at about $2 \frac{1}{2}$ pounds. The secretion of saliva is

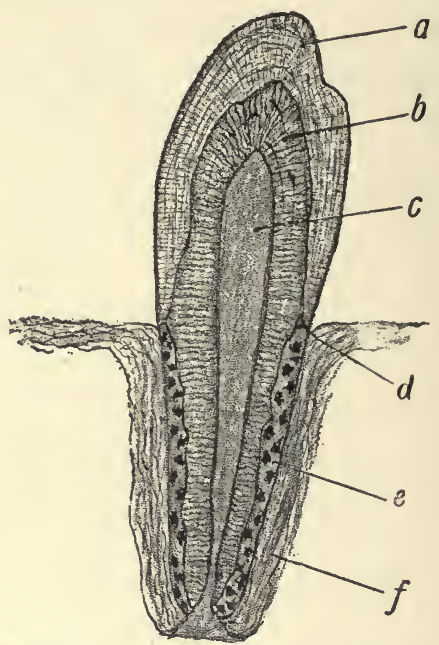

FIG. 76.-Tooth: $a$, Enamel; $b$, dentin; $c$, pulp-cavity; $d$, junction of enamel and cementum; $e$, cementum; $f$, alveolar periosteum (Leroy).

influenced through movements of mastication, by dry substances, and by speaking; moreover, saliva is poured

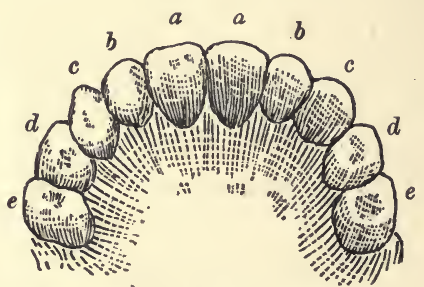

FIG. 77.-Diagram showing the temporary teeth: $a$, Central incisors; $b$, lateral incisors; $c$, canines; $d$, anterior molars; $e$, posterior molars (J. P. C. Griffith).

out in great quantities through the influence of anything which excites taste. 
The Teeth.-The teeth are thirty-two in number. There are sixteen in each jaw, divided into four incisors, or cutting teeth, two canines, four bicuspids, and six molars, or grinding teeth. Each tooth consists of a crown, covered with enamel, a neck, and a root, surrounded by the crusta petrosa, a thin layer of bone, em-

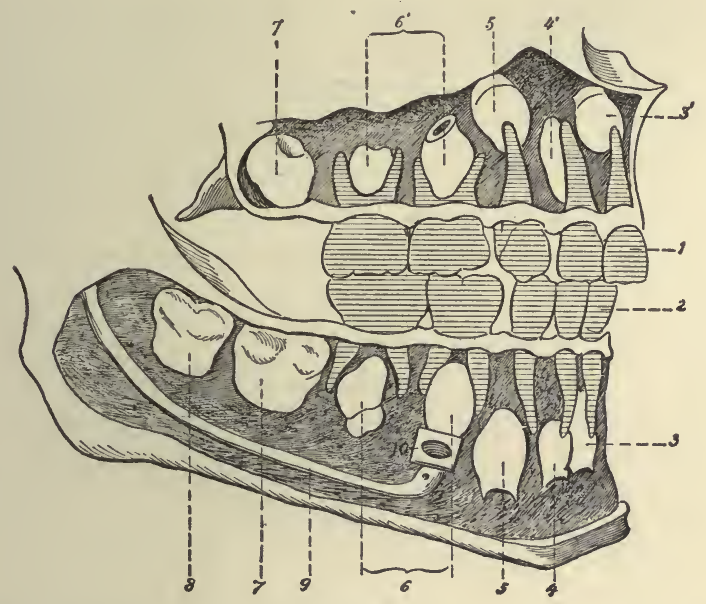

FIG. 78. - Schema showing the temporary and permanent teeth in a child five years old (right side): I, Temporary teeth of the upper jaw; 2 , the five temporary teeth of the lower jaw ; $3,3^{\prime}$, permanent median incisors ; $4,4^{\prime}$, permanent lateral incisors ; $5,5^{\prime}$, permanent canines ; $6,6^{\prime}$, the four permanent bicuspids; $7,7^{\prime}$, first molar; 8 , second molar of lower jaw in its alveolus (in the upper jaw the second molar is not yet formed); 9 , inferior dental canal; ro, orifice of inferior dental canal (after Testut).

bedded in the alveolar process formed by the jaw bones. A section through a tooth shows that it is composed of dentine, in the center of which is the pulp cavity, containing blood-vessels and nerves.

Temporary Teeth.-The temporary teeth of children are twenty in number-ten upper and ten lower. 'They appear at about the following ages, the lower teeth usually 
being erupted first. For convenience a table is appended:

The first four central incisors . ... 5 to 7 months.

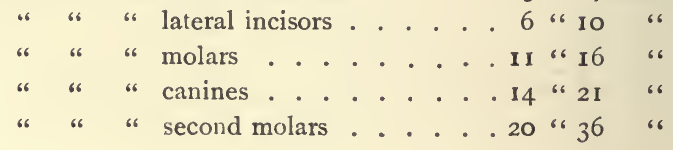

Those lettered $a$ (Fig. 77) are the central incisors; $b$, the lateral incisors; $c$, the cuspids; and $d$ and $e$, the temporary molars. All but the last $(d$ and $e$ ) are replaced by permanent teeth bearing the same names. In the permanent set the first and second bicuspids supplant the temporary molars. By referring to the illustration (Fig. 78 ), it will be seen that while the ten temporary teeth are visible in the mouth, the permanent teeth are being formed within the alveolar margin of the jaws.

The permanent teeth appear in the following order:

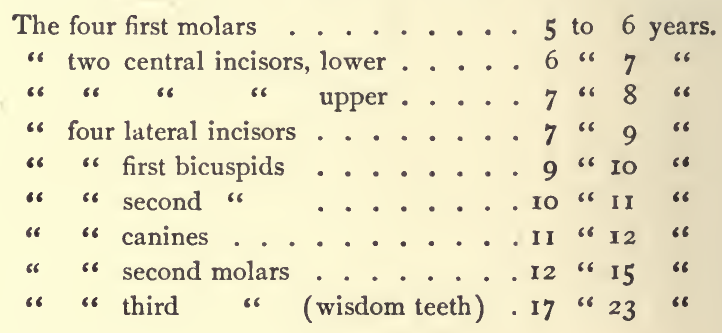

Deglutition.-Deglutition, or swallowing, is the act by which food is forced from the mouth into the stomach. The process may be divided into three stages:

In the first stage, which is entirely voluntary, the mouth is closed and respiration momentarily suspended. The tongue, placed against the roof of the mouth, arches upward and backward, and forces the bolus of food into the fauces. 


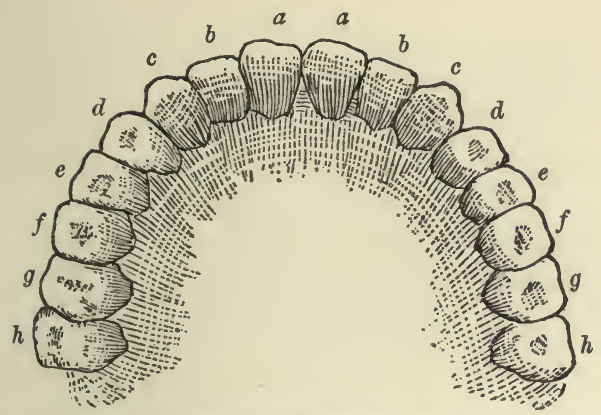

FIG. 79.-Diagram showing the permanent teeth : $a$, Central incisors; $b$, lateral incisors; $c$, canines; $d$, first bicuspids ; $e$, second bicuspids; $f$, first molars; $g$, second molars; $h$, third molars (J. P. C. Griffith).

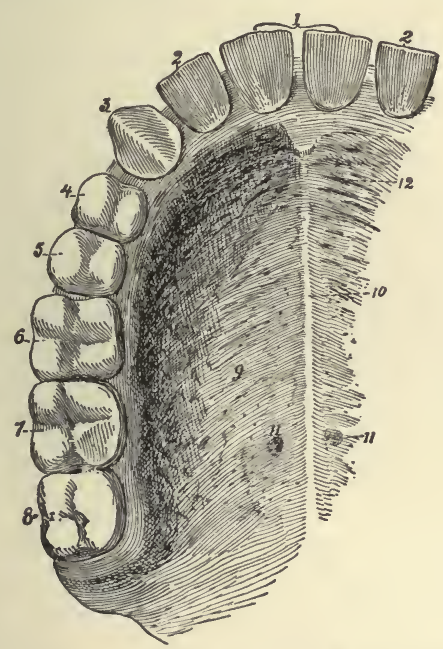

FIG. 80.-The palate and superior dental arch (right side): I, Median incisors; 2, lateral incisors; 3 , canine; 4, first bicuspid; 5, second bicuspids; 6 , first molar; 7 , second molar; 8 , wisdom tooth ; 9 , mucous membrane of the hard palate continuous, behind, with that of the soft palate; Io, the anteroposterior raphé of palate; II, pits on each side of the raphé perforated with the orifices of glands; 12 , anterior rugosities of the mucous membrane (after Testut).

In the second stage, which is entirely reflex, the palate is made tense and directed upward and backward by the 
levatores palati and tensor palati muscles; the bolus is grasped by the superior constrictor muscle of the pharynx and rapidly forced into the esophagus. The food is prevented from entering the posterior nares by the uvula and the closure of the posterior half-arches; from entering the larynx by its ascent under the base of the tongue and the closure of the epiglottis.

In the third stage the longitudinal and circular muscular fibers, contracting from above downward, propel the bolus into the stomach.

\section{THE ESOPHAGUS AND STOMACH}

The esophagus is a tube extending from the lower end of the pharynx, or cavity of the throat, to the stomach, passing through the diaphragm. It is 9 inches long, and consists of two layers of muscle lined with mucous membrane. By contraction of these muscles the food is forced along the tube to the stomach.

The stomach is situated in the left side of the upper portion of the abdomen. Its left extremity is in contact with the diaphragm, and its right is overlapped by the liver. It has two openings: one connected with the esophagus, called the cardiac orifice; the other connected with the upper portion of the intestine, called the pyloric orifice.

It is composed of three coats or membranes: the exterior or serous coat invests every part of this important organ; the middle coat is composed of three layers of muscular fibers, one set of which is arranged longitudinally, the other, circularly; the interior coat is called the mucous, and is arranged in rugæ or folds. The stomach is provided with numerous small glands that secrete the gastric fluids.

The Gastric Juice.-The gastric juice is of an acid character, and possesses very great solvent powers. The 
presence of food in the stomach excites the gastric glands to pour forth their secretion into the stomach. By the

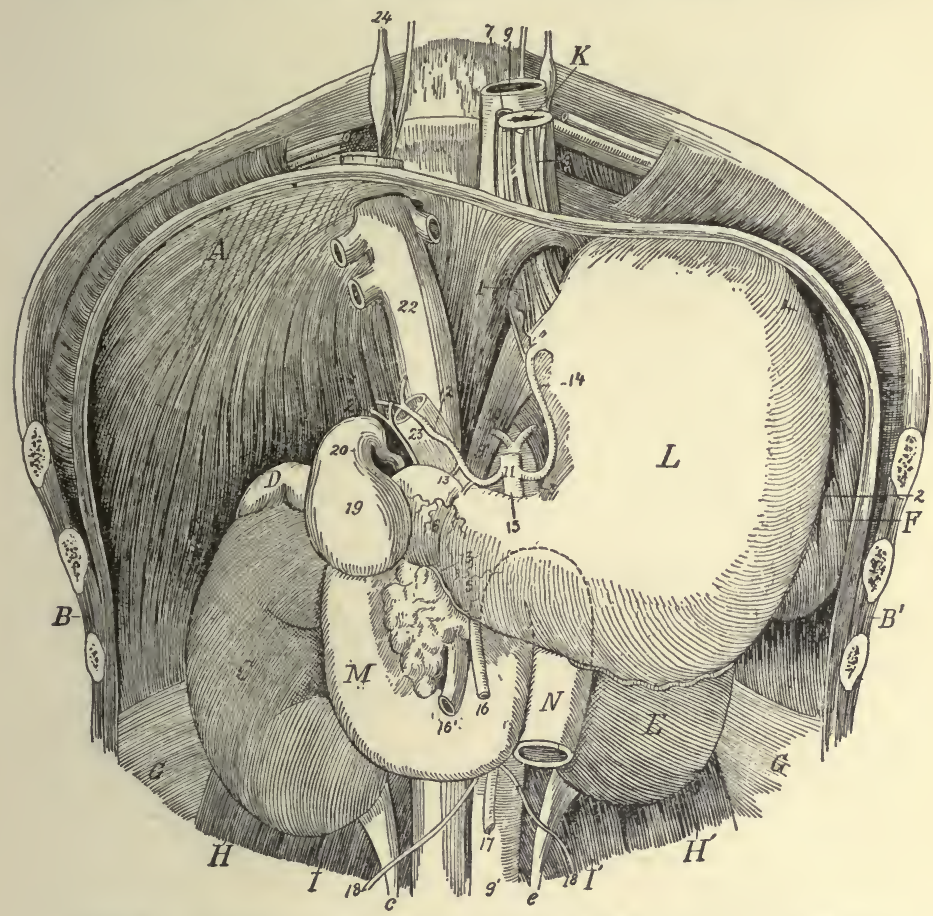

FIG. 8I.-Stomach in place after removal of liver and mass of intestines: $A$, Diaphragm ; $B, B^{\prime}$, thoraco-abdominal wall; $C$, right kidney with $c$, its ureter; $D$, right suprarenal capsule; $E$, left kidney with $e$, its ureter; $F$, spleen; $G$, aponeuroses of transversales; $H, H^{\prime}$, quadrati lumborum ; $I, I^{\prime}$, psoas muscles; $K$, esophagus; $L$, stomach; $M$, duodenum; $N$, origin of jejunum. I, cardia; 2 , greater curvature ; 3 , lesser curvature; 4 , great tuberosity or fundus; 5 , small tuberosity or antrum of pylorus; 6 , pylorus; 7 , right vagus ; 8 , left vagus ; 9 , thoracic aorta ; $9^{\prime}$, abdominal aorta ; 10 , inferior diaphragmatic arteries; II, celiac axis; I2, hepatic artery; I3, right gastro-epiploic; $I 4$, coronary artery; 15 , splenic artery; I6, I6', superior mesenteric artery and vein; 17 , inferior mesenteric artery; 18 , spermatic artery ; 19, gail-bladder ; 20 , cystic duct ; 21, hepatic duct ; 22, inferior vena cava; 23, portal vein; 24, great sympathetic (Testut).

violent mechanical motions of this organ, performed by its muscles whose fibers are so diverse in direction, the 
gastric juice becomes thoroughly mixed with the food, while the digestion of the latter goes rapidly on.
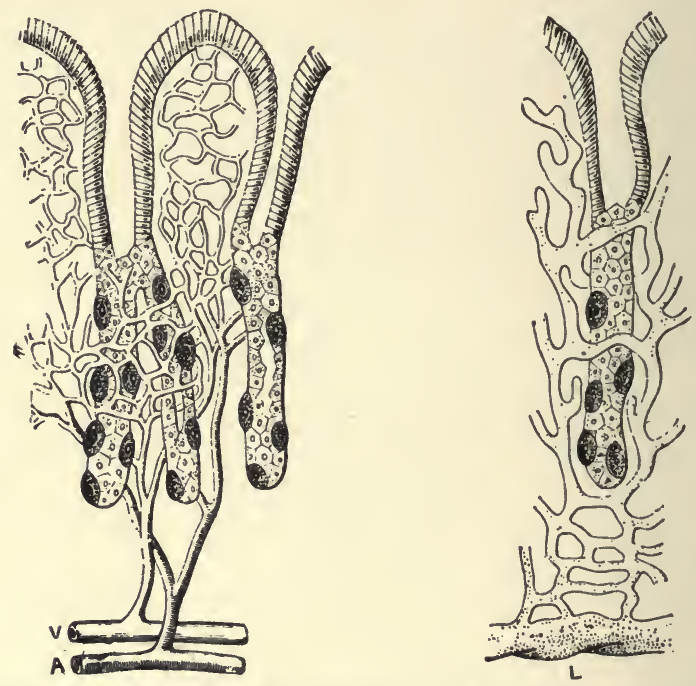

FIG. 82.-Cardiac glands: Diagram showing the relation of the ultimate twigs of the blood-vessels ( $V$ and $A$ ), and of the absorbent radicals $(L)$ to the glands of the stomach, and the different kinds of epithelium-namely, above, cylindrical cells; small pale cells in the lumen; outside which are the dark ovoid cells (Yeo).

The gastric juice is a clear, colorless fluid-acid reaction, with a specific gravity of 1005 ; its composition is as follows:

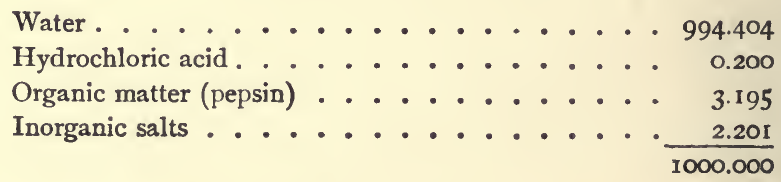

It will be noted that the water forms the largest part of this fluid, and serves to hold the other ingredients in solution. The hydrochloric acid occurs in a free state, and is secreted during the digestive action. It varies in 
quantity. The pepsin is associated with the enzymes of the secretions. Besides pepsin, a second ferment, termed rennin, exists in the gastric juice, which has the power of coagulating the caseinogen of milk. By this action the caseinogen is split into insoluble casein and soluble albumin. Calcium phosphate is essential to the action of this enzyme (Brubaker).

The gastric glands are embedded within the mucous membrane in enormous numbers. They are tubular in character, as may be seen in Fig. 82, which represents the circulation within the glands, as well as the tubules and absorbent radicals.

The principal action of the gastric juice is to transform the different proteid principles of the food into peptones, which is due to the hydrochloric acid and pepsin; when the albumins come in relation with the acid they are converted into parapeptones, and in a short period of time they are converted into albuminose with the aid of pepsin; finally, they are converted into peptones and absorbed.

\section{THE INTESTINES}

The small intestine is the narrowest part of the digestive tract, occupying the central and lower parts of the abdominal and pelvic cavities. It is about 25 feet in length, and is divided, for descriptive purposes, into three parts-the duodenum, jejunum, and ileum. It is suspended from the spine by a fold of peritoneum called the mesentery.

The large intestine extends from the ileum to the anus, and is about 5 feet in length, and is also divided, for purposes of description, into three parts - the cecum, colon, and rectum.

The Small Intestine.-The Duodenum.-This portion is somewhat greater in diameter than the remainder of the small intestine. It received its name from the fact 
that it is about twelve finger-breadths in length. Above, it commences at the pylorus, and ascends obliquely backward to the under-surface of the liver. It then descends perpendicularly in front of the right kidney, and passes transversely across the lower portion of the spinal column, behind the colon, and terminates in the jejunum. The ducts from the liver and pancreas open into the perpendicular portion, about 6 inches from the stomach.

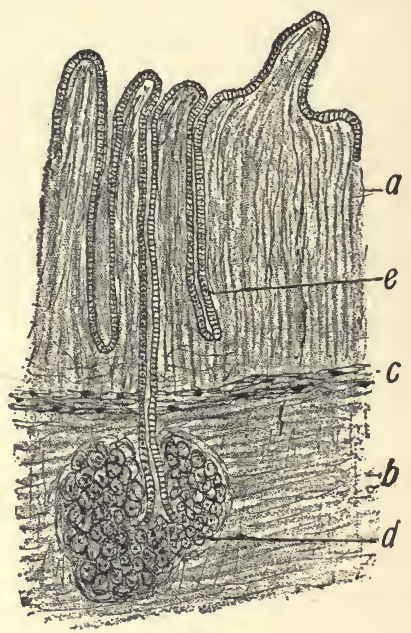

FIG. 83.-Duodenum : $a$, Mucosa; $b$, submucosa; $c$, muscularis mucosæ; $d$, Brunner's gland; $e$, crypts of Lieberkühn (Leroy).

The Jejunum. - The jejunum is continuous with the duodenum, and includes the upper two-fifths of the remainder of the small intestine, lying chiefly in the umbilical region and left iliac fossa. It has a pinkish tinge, and is thicker than the remainder of the small intestine.

The ileum is smaller, thinner in texture, and somewhat paler than the jejunum. There is nothing to mark the termination of the one or the commencement of the other 
of these portions. The ileum terminates, at an obtuse angle, near the right pelvic or haunch bone by a valvular opening into the colon, which is guarded by the ileocecal valve. This arrangement prevents the passing of substances from the colon into the ileum. The jejunum and ileum are surrounded above and at the sides by the colon.

The small intestine, like the stomach, has three coats (some writers include a fourth, or submucous coat). The

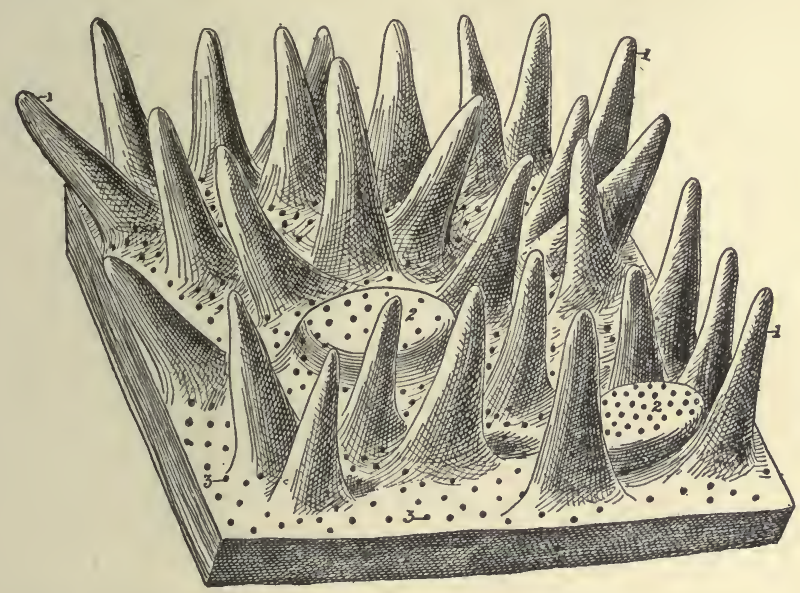

FIG. 84.-Mucous membrane of the jejunum, highly magnified (schematic): I, I, Intestinal villi; 2,2 , closed or solitary follicles; 3,3 , orifices of the follicles of Lieberkühn (Testut).

inner or mucous coat is thrown into folds or valves. In consequence of this valvular arrangement the mucous membrane is more extensive than the other tissues, and gives a greater extent of surface with which the aliment comes in contact. Protruding from the surface of the mucous coat are an immense number of projections which are called villi and give the surface the appearance of velvet; for this reasonthis membrane is sometimes called the villous coat. 
The small intestine is beset with numerous small glands, called Peyer's glands or patches; they are most numerous in the lower two-thirds of the ileum. These patches are liable to become ulcerated in chronic dysentery, and are the seat of the ulceration of the intestine that occurs in typhoid or enteric fever; in fact, this is the supposed location and seat of the disease.

The Large Intestine.-The cecum (cul-de-sac), or blind pouch, as it is sometimes called, is the commencement of the large intestine. It is closed at the end, and has projecting from its junction with the ileum a slender, worm-like appendage, called the "appendix vermiformis."

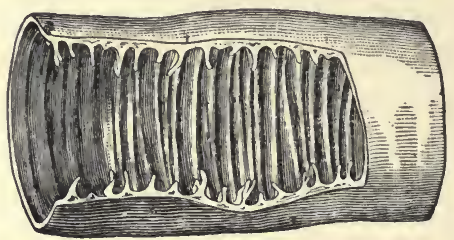

FIG. 85.-Portion of the wall of the small intestine, laid open to show the valvulæ conniventes (Brinton).

This structure is situated in the right iliac region, and is about three or four finger-breadths in length. The cecum, like the other portions of the intestine, has a pouch-like arrangement, caused by the peculiar position of the fibers of its muscular coat.

The appendix vermiformis is a narrow, blind, wormlike tube, from 3 to 6 inches long, springing from the lower and back part of the cecum and held in a variable position by a peritoneal fold. Its function as yet has not been determined. It is susceptible to extensive inflammation, resulting in many instances in suppuration, which requires immediate surgical intervention for the safety of the individual. 
The ileocecal valve (Fig. 86) is formed by two longitudinal semilunar folds of mucous membrane at the termination of the ileum in the cecum, opening toward the large intestine and guarding against reflux from the large into the small intestine.

The colon is divided into three parts- the ascending, the transverse, and the descending. The ascending colon passes upward from the right hip-bone to the under surface . of the liver. It then bends inward and crosses the upper part of the abdomen, below the liver and stomach, to the

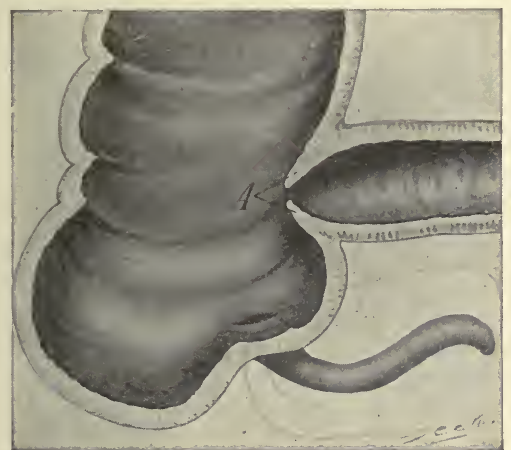

FIG. 86.-The cecum, vermitorm appendix, and ileocecal valve: $A$, The ileocecal valve (Campbell).

left side, under the name of transverse colon. At the left side it turns and descends to the left hip-bone, and is here called the descending colon. At this point it makes a peculiar curve upon itself, called the "sigmoid flexure," from its resemblance to the letter $S$, On its internal surface the colon has many sac-like folds, which serve to retain the contents in its passage for a long period.

The rectum is the lowermost or last portion of the large intestine, and terminates in the anus; at this point it is surrounded by a sphincter or circular muscle that keeps the orifice closed except during defecation. 
Peristalsis is the peculiar worm-like motion of the intestines, by means of which its contents are kept in motion and become mixed with the digestive fluids. It is produced by a compressing action of the circular fibers combining with a shortening action of the longitudinal ones.

Besides the organs just described as being concerned in the nutrition of the body, we will consider here those structures whose function is the absorption of the digested

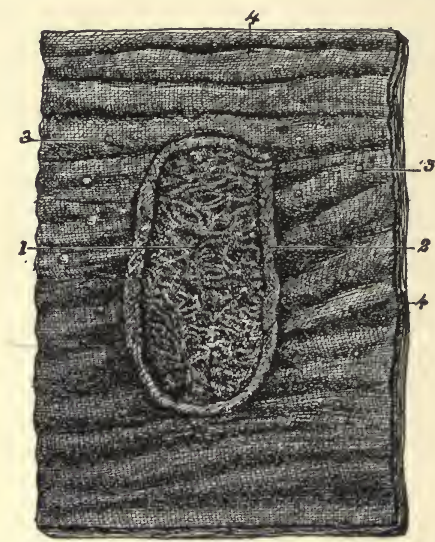

FIG. 87.-Mucous membrane of the jejunum: I, Peyer's patch; 2, its border; 3 , solitary follicles; 4,4 , valvulæ conniventes (Testut).

food. These are the lymphatics of the small intestine, known as the lacteals, the villi, and the thoracic duct. (See p. 175.) Of these, the villi have already been described.

Intestinal digestion is an important and complex function, requiring several important secretions-namely, the pancreatic juice, the bile, and the intestinal juice, the first two of which are the most important. They mingle together in the small intestine and act upon the food, not in succession, but simultaneously. 
The material passed to the intestine during stomach digestion, called chyme, is slightly acid in reaction. As it passes through the pylorus into the intestine, it excites therein a secretion of the intestinal fluids. These latter have an alkaline reaction, which neutralizes the substances passed from the stomach; the nutriment which has not been absorbed by the stomach is taken up by the absorbents of the intestine and sent to the several parts of the system, as will be noted under the Lacteals.

It is supposed that in the intestines starchy substances are converted into dextrose. This applies especially to the conversion of cane-sugar, maltose, and lactose into dextrose, by which process they are prepared for absorption.

Pancreatic juice is secreted by the pancreas, the duct of which opens into the intestine (duodenum). This juice is a transparent, colorless fluid, strongly alkaline, with a specific gravity of r.040. Its composition is as follows:

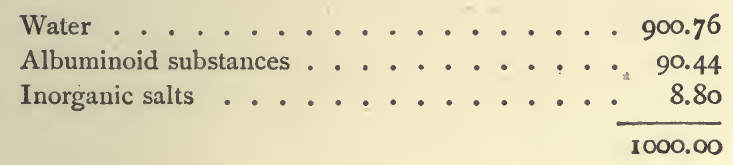

It is the function of the pancreatic juice to convert starch into maltose, and albumin into albumose, and finally into peptone; the trypsin contained in it converts the hemipeptones into leucin and tyrosin. Pancreatic juice has a specific action upon fats, which it emulsifies. This action is rapid, and depends upon the alkaline substances with which it is combined. It is supposed that the neutral fats are acted upon, and converted into fatty acids and glycerin by a ferment called steapsin. The pancreatic juices increase the peristalsis of the intestines through the glycerin which is produced. 
The individual actions of the several ferments of the pancreatic juice are as follows: Amylopsin is the diastic ferment of the pancreatic juice; it converts starches into maltose and glucose. It transforms glycogen into grape sugar, and dissolves cellulose. This action may take place in an alkaline, neutral, or acid medium.

Trypsin converts albumin into peptones in alkaline medium, also in slightly acid media.

Steapsin converts fat into fatty acids and glycerin.

The bile is an important secretion, whose action is to prepare the food for absorption. It is of a golden color, and has an alkaline reaction and a specific gravity of $\mathbf{1} .020$. Its composition:

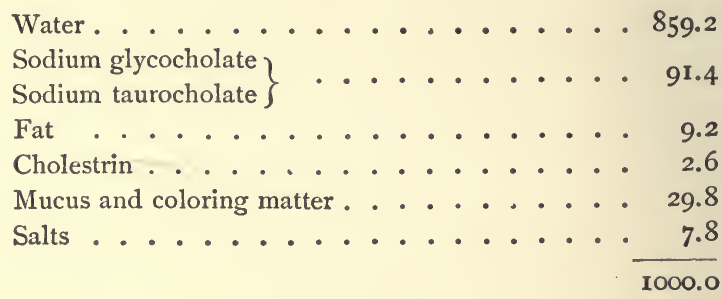

The function of the bile is to assist in the emulsification of the fats and to aid in their absorption. It prevents putrefactive changes in the food. It stimulates the secretion of the intestinal glands and excites peristaltic action of the bowels. The total amount secreted in twenty-four hours is estimated to be $2 \frac{1}{2}$ pounds.

The intestinal juice (succus entericus) is secreted from the small glands of the intestines (glands of Lieberkühn). It resembles light Rhine wine in color, and is strongly alkaline from the carbonate of sodium it contains. The constituents are albumin and mucin. 


\section{THE LACTEALS}

The lacteals are minute vessels that commence in the villi of the small intestine. They pass between the layers of the mesentery to small glands, which they enter. The first range of glands collects many small vessels, and transmit a few larger branches to a second range of glands; and, finally, after passing through several successive ranges of these glandular bodies, the lacteals, diminished in number and increased in size, proceed to the enlarged portion of the thoracic duct, into which they open. They are more numerous in the upper portion of the small intestine.

The absorption of digested materials is accomplished by the lymphatics or lacteals of the intestines which form part of this great system. The absorption takes place in the villi, which are part of the mucous coat of the small intestine. Passing through the epithelial layer, the lymph comes at once in contact with the capillaries of the vascular network.

Lymphatic absorbent vessels are found in every part of the body-in the glandular system, muscular organs, and mucous membranes. The lymph is a colorless or slightly yellowish, transparent liquid.

The lymphatic vessels of the intestines originate in the substance of the villi. They form plexuses, and continue to build up in size and diminish in number until they finally terminate in a saccular dilatation called the receptaculum chyli, which is the beginning of the thoracic duct.

The products of digestion, which are taken up by the blood-vessels and lymphatics of the intestines, pass by two different routes into the general circulation. The blood of the portal system, containing albuminose, sugar, and molecular fat, is carried at once to the liver, where it traverses the capillary vessels of this organ before reaching the ascending vena cava and right side of the 
heart. The chyle, on the other hand, containing also a large portion of fatty ingredients, passes by the thoracic duct, and mingles with the return current of the venous blood in the subclavian vein, where it undergoes an immediate transformation. In this transformation tle fatty matter loses its distinctive character and is no longer

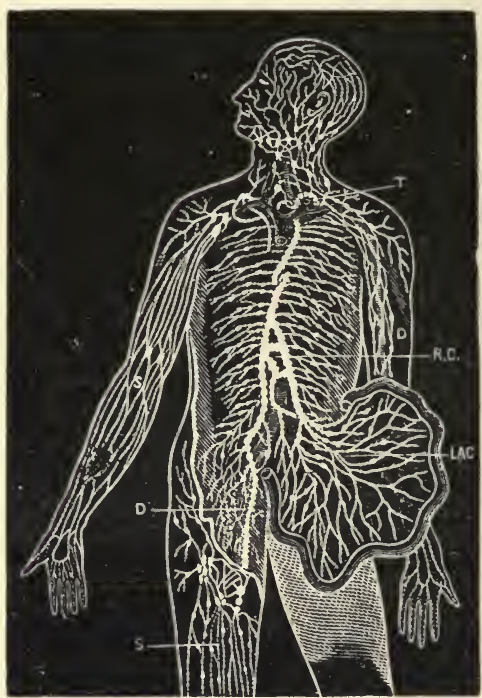

FIG. 88.-Diagram showing the course of the main trunks of the absorbent system: the lymphatics of lower extremities (D) meet the lacteals of the intestines (LAC) at the receptaculum chyli (R.C.), where the thoracic duct begins. The superficial vessels are shown in the diagram on the right arm and leg (s), and the deeper ones on the left arm (D). The glands are here and there shown in groups. The small right duct opens into the veins on the right side. The thoracic duct opens into the union of the great veins of the left side of the neck (T) (Yeo).

visible as oleaginous molecules. The nutritive elements of the food, prepared for absorption by the digestive process, are taken up into the circulation under the different forms of albuminose, sugar, and chyle, and accumulate as such, at certain times, in the blood; but these conditions are temporary and transitional. The nutritive materials 
soon pass by transformation into other forms, and become assimilated to the pre-existing elements of the circulating fluid. In this way they accomplish, finally, the object of digestion, and replenish the blood by a supply of new material from without.

\section{THE THORACIC DUCT}

The thoracic duct begins in the abdomen by a considerable dilatation which is situated in front of the lower

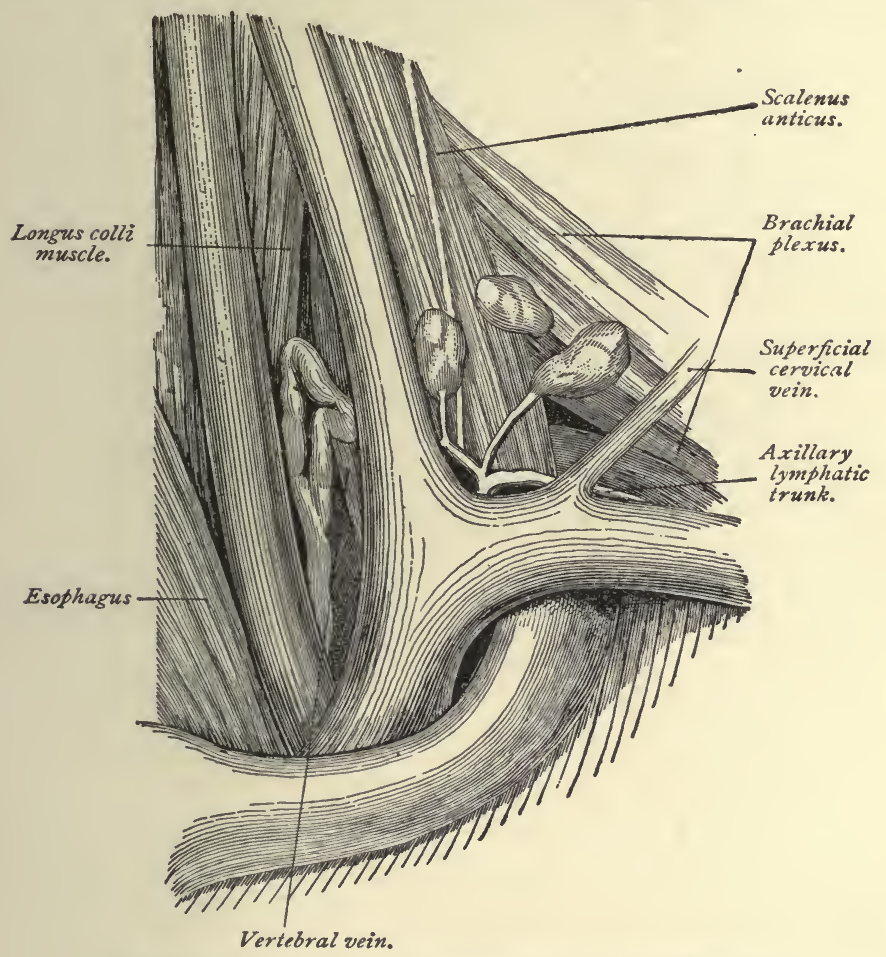

FIG. 89.-Topography of the thoracic duct (Zuckerkandl).

portion of the spinal column. From this point it passes 
through the diaphragm, and ascends to the lower part of the neck. In its ascent it lies anterior to the spine, and by the side of the aorta and esophagus. At the lower part of the neck it makes a sudden turn downward and

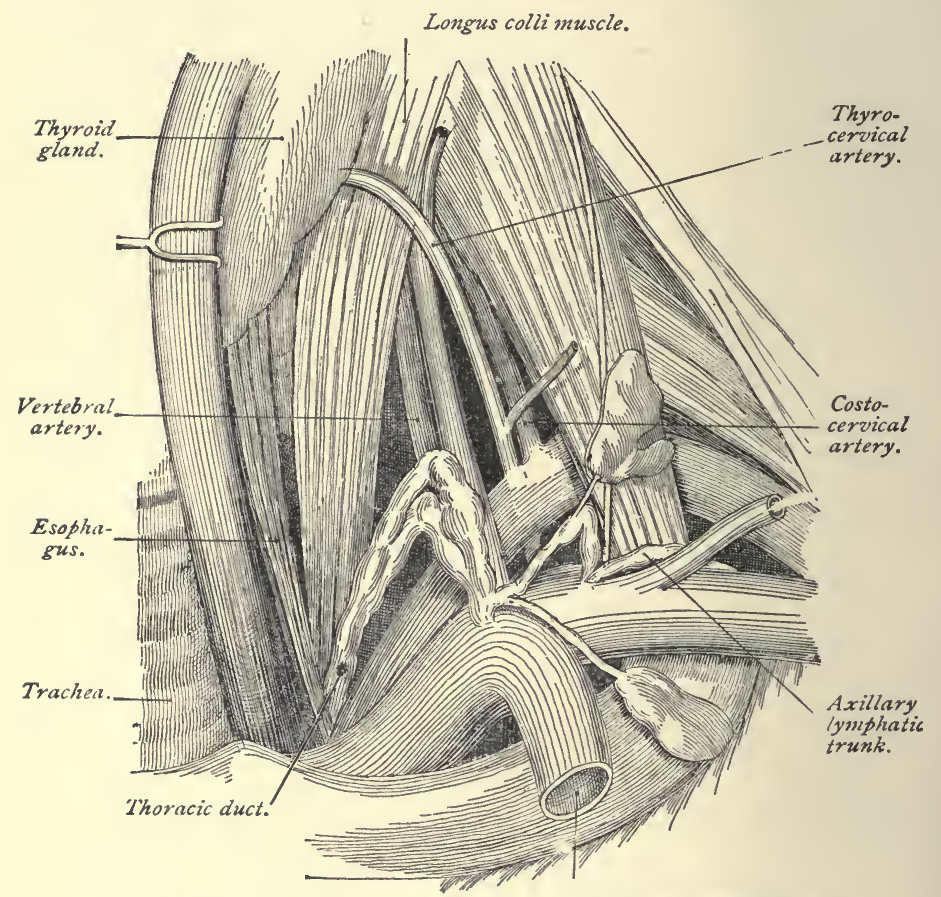

Internal jugular vein.

FIG. 90.-Topography of the thoracic duct (Zuckerkandl).

forward, and terminates by opening into the left subclavian vein. The thoracic duct is equal in diameter to a goose-quill, and at its termination is provided with a pair of semilunar valves, which prevent the admission of venous blood. 


\section{REGIONS OF THE ABDOMEN AND THEIR CONTENTS}

By referring to the illustration (Fig. 9I), you will observe that the abdomen is divided into nine regions; these are: The right hypochondriac, right lumbar, right inguinal, epigastric, umbilical, hypogastric, left hypochondriac, left lumbar, and left inguinal. The division, as will be seen from the illustration, is made by draw-

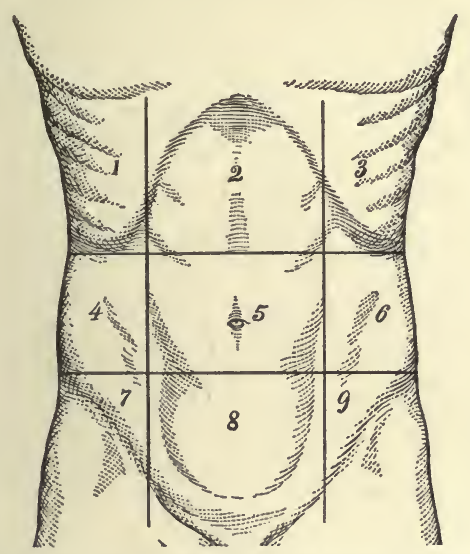

FIG 9I.--1maginary lines drawn upon the surface of the abdomen dividing it into regions: 1 , Right hypochondriac region; 2 , epigastric; 3 , left hypochondriac; 4 , right lumbar; 5 , umbilical ; 6 , left lumbar; 7 , right iliac ; 8, hypogastric; 9, left iliac (Chapman).

ing an imaginary line horizontally between the cartilages of the ninth ribs; another between the crest of the ilia; and two vertical lines from the cartilages of the eighth ribs to the center of Poupart's ligament.

The right hypochondriac region contains the right lobe of the liver, the gall-bladder, the duodenum, the hepatic flexure of the colon, the upper part of the right kidney, and the right suprarenal capsule. 
The epigastric region contains the right two-thirds of the stomach, the left lobe of the liver, the celiac axis, the solar plexus, the pancreas, and part of the aorta, inferior vena cava, vena azygos, and thoracic duct.

The left hypochondriac region contains the splenic end of the stomach, the spleen, the tail of the pancreas, the splenic flexure of the colon, the upper half of the left kidney, and its suprarenal capsule.

The right lumbar region contains the ascending colon, the lower half of the right kidney, and part of the small intestine.

The umbilical region contains the transverse colon, the transverse duodenum, part of the great omentum and mesentery, and part of the small intestine.

The left lumbar region contains the descending colon, the lower half of the left kidney, and part of the small intestine.

The right inguinal region contains the right ureter, the cecum, the appendix vermiformis, and the spermatic vessels of that side.

The hypogastric region contains part of the small intestine, the bladder in children (and when distended, in adults), and the uterus during pregnancy.

The left inguinal region contains the left ureter, the spermatic vessels, and the sigmoid flexure of the colon.

\footnotetext{
REVIEW QUESTIONS

Es sublogus. Intes. Jfum

What organs constitute the digestive system?

What is mastication?

What organs are brought into action in the mastication of foods?

What nerve controls this action?

What is the saliva?

What important enzyme does the saliva contain?

How many temporary teeth are there?

At what ages are they erupted?

Give the number of permanent teeth.
} 
What is deglutition?

What is the stomach?

What important openings does the stomach contain?

How many coats has the stomach?

What is the gastric juice?

What is its composition?

Name the several enzymes of same, and state their several actions.

State the divisions of the small intestine.

Give the location of the duodenum of the jejunum, of the ileum.

What valve is situated at the junction of the small with the large intestine?

What patches are found in the lower portion of the ileum?

What disease is particularly apt to affect these glands?

What secretions are concerned in the digestive process?

What is the pancreatic juice?

What is the composition of the pancreatic juice?

Describe the individual action of the several ferments of the pancreatic juice.

What is the bile, and from what source is it derived?

What is the action of the bile?

State the estimated amount secreted in twenty-four hours and give the color of the bile.

What is produced when the intestines are inflamed?

What are the glands of Lieberkühn?

What secretions are formed from these glands?

How is the large intestine divided as to name?

What important flexure is there in the descending portion?

What is the rectum? In what does it terminate?

What do you understand by peristaltic movements?

What are lymphatics?

Where is the thoracic duct located?

What is provided at its termination? Why?

Where do the lacteals originate?

Through what route do the products of digestion pass in entering the circulation?

What are the lacteals?

Should digestion be interfered with? What would be the result?

What are the regions of the abdomen?

State as nearly as possible what important organs are located in the several divisions. 


\section{CHAPTER VI}

\section{THE EXCRETORY SYSTEM}

WE have considered the circulatory, the respiratory, and the digestive systems, with the various chemic substances that are required to maintain the life, growth, and activity of the body. These substances, entering the current of the blood, are carried to all organs and tissues, and are incessantly combining with the chemic substances of which these tissues are composed. These combinations are not dependent upon chance; each tissue has a special affinity for the chemic substance in the blood that it requires for its own growth and special form of activity; for example, the secretory cell of the liver selects substances from which it can elaborate bile and glycogen; the muscle-fiber assimilates those that will promote the changes upon which the power of contractility depends.

We know that the proteid compounds contain the most essential elements for the formation of all forms of tissue, and that phosphate of lime is a necessary factor in the hardening of bone, but we are utterly ignorant of the process by which each tissue element is enabled to select the particular substance it needs and to reject that which it does not require.

Metabolism.-Our bodies are masses of changing atoms, some of which, we may say, are on the "up grade," to construct the various tissues, and some on the "down grade," to form the waste matters that are the final products of the tissues' activity. These changes, which are going on incessantly while life lasts, are described under 
the general term metabolism; the constructive changes are termed anabolic; the destructive, katabolic.

The final products, then, of the metabolism of the body will be certain waste matters; we shall now proceed to describe the mechanism of the organs by means of which these waste products are eliminated from the body.

The Elimination of Waste Products.-In passing through the blood and tissues of the body the proteids, fats, and carbohydrates are transformed into urea, or some allied product, carbon dioxid, and water, the nitrogen of the urea being supplied by the proteids alone. Many of the proteids contain sulphur, and in some phosphorus in some combination is also present; many of the fats taken as food contain phosphorus. By oxidation these elements are converted into phosphates and sulphates, and are excreted in company with other salts of the body.

Broadly speaking, then, the waste products of the body consist of urea, carbon dioxid, salts, and water. These leave the body by one of three main channels-the lungs the skin, or the kidneys. A portion, it is true, leaves the body by the bowels, for, as we have seen, the feces contain, besides undigested portions of food, substances that have been excreted into the bowels, and are therefore waste products; this amount is, however, very small, and, except in diseased conditions, is not of any special importance. The waste matter discharged relatively by the lungs, skin, and kidneys may be stated to be as follows:

By the lungs: The greater part of the carbon dioxid; a considerable quantity of water.

By the skin: A variable but, on the whole, large quantity of water, a little carbon dioxid, and a small amount of salts. -

By the kidneys: All or nearly all the urea and allied bodies; the greater part of the salts; a large amount of water and a very small quantity of carbon dioxid. 
Having studied, in a previous chapter, the mechanism by which the lungs relieve the blood of carbon dioxid and water, it now remains for us to consider the excretory mechanism of the kidneys and of the skin. The former excretes the urine, while the other urinary organs, the ureters, bladder, and urethra, collect the urine and effect its exit from the body. The mechanism by which the

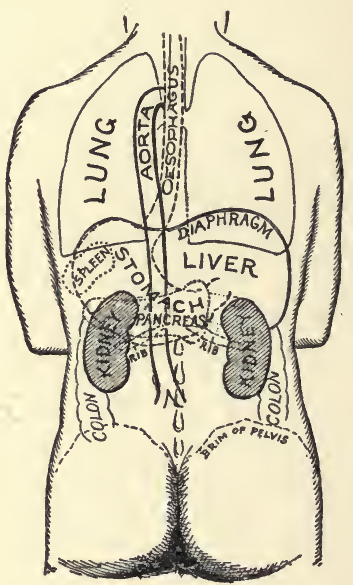

FIG. 92.-Diagram of the relation of kidney to viscera, spine, and surface points (American Text-Book of Surgery).

skin excretes waste products in the form of perspiration will be considered further on.

\section{THE KIDNEYS}

The kidneys are two tubular, secreting glands, placed at the back of the abdominal cavity, one on each side of the lumbar vertebræ. They are bean shaped, the concave side being directed toward the spine, and the convex side outward. Each kidney is about 4 inches long, 2 inches broad, I inch thick, weighs from 5 to 6 ounces, 
and extends from the eleventh dorsal to the third lumbar vertebra. The right kidney is a little lower than the left, in consequence of the large space occupied by the liver. The kidneys are covered by a tough envelope of fibrous tissue called the capsule of the kidney, and are usually embedded in a considerable quantity of fat.

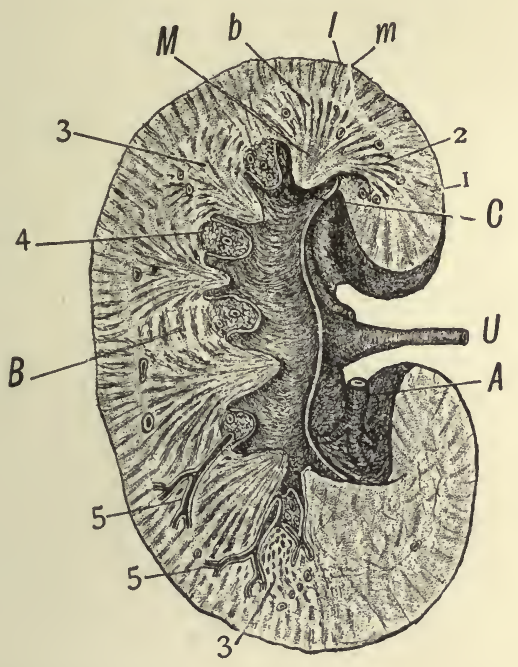

FIG. 93.-Kidney, longitudinal section, exhibiting general relations of microscopic details (after Piersol): $A$, Renal artery; $U$, ureter; $C$, one of the calices, into which a papilla projects; $\mathrm{r}$, cortex containing labyrinth $(l)$ and medullary rays $(m) ; 2$, medulla; $M$, Malpighian pyramids, some obliquely cut at 3,$3 ; b$, boundary layer; $B$, columns of Bertini ; 4 , masses of adipose tissue $; 5,5$, branches of renal artery (after Henle).

Structure of the Kidneys.-The kidney is made up of an outer part, the cortex, and an inner part, the medulla. Its substance is composed of a mass of uriniferous tubules and Malpighian bodies. The Malpighian bodies (Fig. 94) are located in the cortex of the kidney near the surface, and consist of a tuft of blood-vessels, the glomerulus, and an expansion of the uriniferous tubule, which forms a covering about the glomerulus. The uriniferous tubules, 


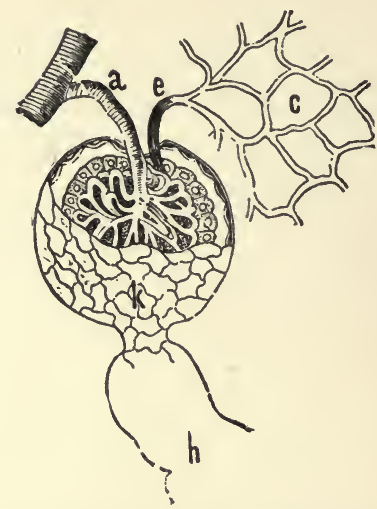

FIG. 94.-A Malpighian body or corpuscle; $a$, Afferent artery ; $e$, efferent vessel ; $c$, capillaries ; $k$, commencement of uriniferous tubule; $h$, uriniferous tubule (Leidy).

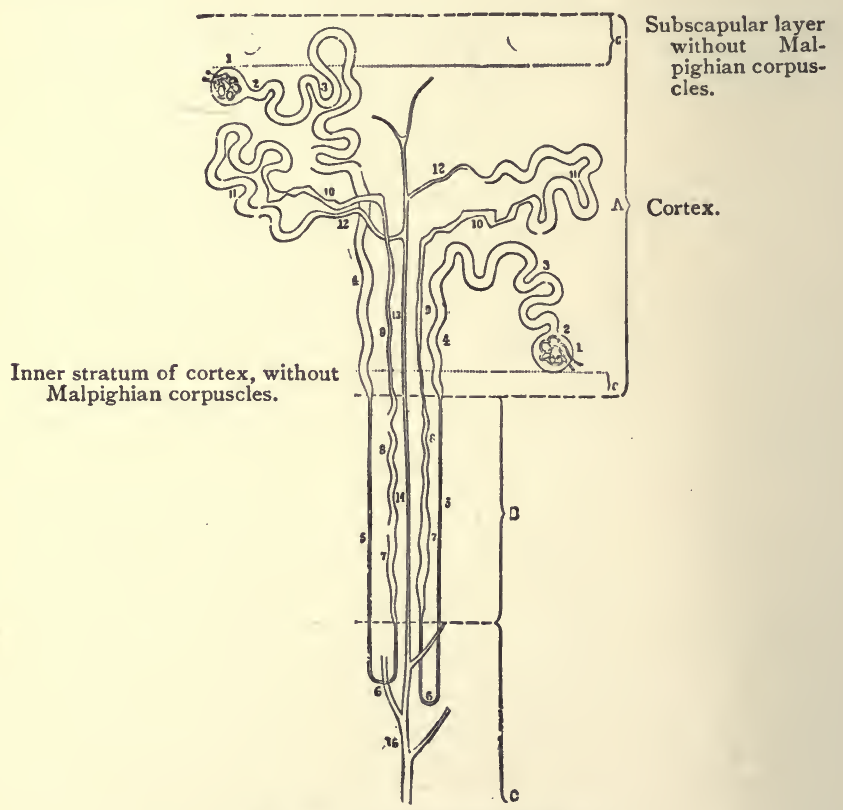

FIG. 95.-Uriniferous tubule. 
after leaving the Malpighian body, twist and turn several times, finally emptying into the ureter (Fig. 95).

By the arrangement of blood-vessels described above, the arterial blood is brought directly to the glomerulus. It is during its circulation through the glomerulus that the blood gets rid of some of its fluid constituents, while the

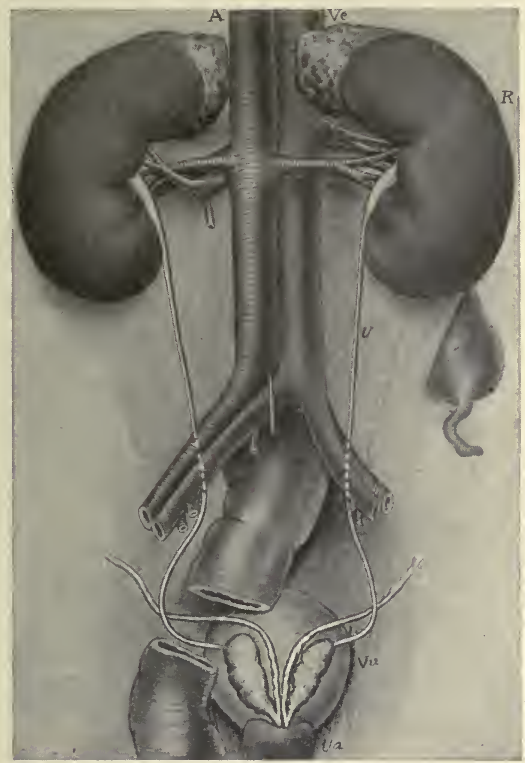

FIG. 96.-The kidneys, ureters, bladder, and their vessels viewed from behind: $R$, Right kidney; $U$, ureter; $A$, aorta; $A r$, right renal artery; $V e$, vena cava inferior; $V r$, right renal vein; $V u$, bladder; $U a$, commencement of urethra (Campbell).

waste products are excreted by, the uriniferous tubules. The waste matter, in the form of water, carbon dioxid, salts, and urea, passes into the tubules, and is passed off from the kidney in the form of urine.

The Blood=supply of the Kidneys.-For an organ of its size, the kidney is abundantly supplied with 
blood. This is derived from the renal artery, which, as we have seen in the description of this structure in a previous chapter, comes directly from the abdominal aorta.

\section{THE URETERS}

The ureters are the excretory ducts of the kidneys. They arise in the middle of the concave side, or hilus, of each kidney, and proceed obliquely downward and inward through the lumbar region of the abdomen into the pelvis, to open obliquely by two constricted orifices into the base of the bladder. Each ureter is from 16 to 18 inches long, of the diameter of a goose-quill, and is made up of muscular tissue lined by mucous membrane. The muscular coat is arranged in two layers-an outer circular and an inner longitudinal layer. Outside the muscular coat is a layer of fibrous connective tissue, carrying the blood-vessels and nerves with which the tube is supplied.

\section{THE BLADDER}

The bladder is a musculomembranous sac that serves as the reservoir for the urine. It is situated in the pelvic cavity, behind the pubes, and is held in position by ligaments. During infancy it is conic in shape, and projects above the upper border of the pubes into the hypogastric region. In the adult, when quite empty, it is deeply placed in the pelvis. When slightly distended it has a rounded form, but when greatly distended it is ovoid in shape, and rises to a considerable height in the abdominal cavity. When moderately distended, it measures about 5 inches in length and 3 inches across. The amount of urine which the bladder ordinarily contains is about I pint.

In structure, the bladder consists of involuntary muscular tissue lined by a strong mucous membrane, and 
covered partially by a serous coat derived from the peritoneum. The muscular coat has three layers, the principal fibers of which run longitudinally and circularly, the circular fibers being collected into a layer of some thickness around the constricted portion or neck, where the bladder becomes continuous with the urethra. These circular fibers around the neck form a sphincter muscle that normally is in a state of contraction, relaxing only at intervals, when the accumulation of urine within the bladder renders its expulsion necessary.

The Urethra.-The urethra is a narrow, membranous canal, about $1 \frac{1}{2}$ inches in length in the female, and extending from the neck of the bladder to the external orifice, or meatus urinarius. It is situated beneath the symphysis pubis, and is embedded in the anterior wall of the vagina. It runs obliquely downward and forward, its course being slightly curved, the concavity directed forward and upward. While it admits of considerable dilatation, its normal diameter is about $\frac{1}{4}$ inch. It is lined by a mucous coat that is continuous, externally, with that of the vulva, and, internally, with that of the bladder. The external muscular coat is also continuous with that of the bladder, but between the mucous and muscular coats is a layer of thin, spongy tissue, containing a network of large veins. In the male, the urethra extends from the neck of the bladder to the meatus urinarius at the end of the penis, measuring 8 or 9 inches. It is divided into a prostatic portion, a membranous portion, and a spongy portion. The latter portion is the longest, being contained in the corpus spongiosum of the penis (see Fig. 156). (By referring to the illustrations, Figs. I45, I46, the structures that make up the urinary organs may be clearly seen.)

Micturition.-This act is normally caused by the accumulation of urine within the bladder. The accumu- 
lation stimulates the muscular walls to contract, the resistance of the sphincter at the neck of the bladder is overcome, and the urine is ejected through the urethra.

Involuntary micturition may occur as a result of spinal injury, involving the nerve-centers that send nerves to the bladder. It may also be due to a want of tone in the muscular walls, or it may result from abnormal irritation.

The Urine.-General Character of the Urine.Normal urine may be described as a transparent, watery fluid, of a pale yellow color, acid reaction, a specific gravity of IOI 8 to 1020, and possessing an odor that can only be described as characteristic or urinous. Each one of these characters is subject to some variation within the limits of health as well as in disease.

The transparency of urine may be diminished in health by the presence of mucus derived from the genito-urinary tract, or by the deposits of salts. In disease the urine may become clouded by the presence of pus.

The color of urine depends mainly upon the amount of water it contains; also upon a diminution or increase of the coloring matter. In hysteria, in which the urine is copious in amount, the color is very light, while in fevers, in which it is scanty, the color is very high. It may take on abnormal color as the result of the ingestion of certain foods or medicine, or this may be due to a diseased condition.

The reaction of urine should always be tested from a collection of urine passed during twenty-four hours, for it is affected by diet and exercise; the reaction of mixed urine is normally acid.

The specific gravity of urine depends upon the amount of solid waste matter present in the urine. In health it may vary from IOI5 to I025. When the solids are dissolved in a large amount of water, the specific gravity will naturally be lower than when, from a deficiency of water, 
the urine is more concentrated. In the disease known as diabetes mellitus the specific gravity of the urine is considerably heightened, owing to the presence of sugar. The specific gravity is of greatest value when it is obtained from a twenty-four-hour collection.

The Composition of Urine.-The chief constituents of normal urine are water, urea, uric acid, coloring matter, and salts. Of these constituents, urea is by far the most important, for it is the chief waste product of the body. To eliminate urea is the special work of the kidneys, and if, for any reason, they fail to do their work, the accumulation of the urea in the system eventually leads to the termination of life. Urea is the final product of all proteid substances, and, consequently, a diet rich in proteids will increase the amount of urea in the system.

Of the salts, sodium chlorid occurs in the largest quantity. In certain inflammatory diseases, in which it is needed by the blood, it sometimes disappears temporarily from the urine.

When the kidneys are diseased, it is customary for physicians to lighten their work as far as possible by regulating the diet.

The chief abnormal constituents that are liable to appear in the urine are albumin and sugar; the former gives rise to a condition known as albuminuria; the latter, to the disease called diabetes.

The normal quantity of urine passed in twenty-four hours is from 40 to 50 ounces, or about 3 pints. This amount will vary in health according to the condition of the skin and the amount of fluid taken into the body. The excretion of water by the kidneys is closely related to a similar process conducted by the skin. When the body is exposed to cold the blood-vessels in the skin are constricted, and the discharge of water in the form of sweat is checked; at the same time the blood-vessels of the kidneys 
are dilated, there is a full and rapid stream of blood through the glomeruli, and an increased flow of urine results. On the other hand, when the body is exposed to warmth, the cutaneous vessels are widely dilated and free perspiration occurs, while the renal vessels being constricted, only a small and slow stream of blood trickles through the glomeruli, and the urine that is secreted is scanty in amount.

The effect of secretion, however, is governed largely by the amount of fluid absorbed through the alimentary canal; an increased secretion of water always follows an ordinary meal, and when large quantities of water are drunk, the amount of urine is correspondingly increased.

\section{THE SUPRARENAL CAPSULES}

The suprarenal capsules are two small, flattened bodies, of a yellowish color, one situated immediately above each kidney. Having no excretory duct, they are usually classified with the ductless glands. Each organ is invested by a fibrous capsule that sends septa into the glandular substance; these septa form a framework for the soft, pulpy substance of the gland, and within the spaces of this framework are groups of cells.

The suprarenal capsules are abundantly supplied with blood-vessels, nerves, and lymphatics, and contain remarkable coloring matters. When these organs become diseased, the skin frequently becomes "bronzed," from an increase of pigment or coloring-matter. Their special function is as yet unknown.

\section{THE SKIN}

Having described the mechanism by which the lungs eliminate carbon dioxid and water from the body, and the 
manner in which the kidneys relieve it of urea, salts, and water, it now remains for us to consider the part played by the skin in eliminating water and a certain amount of carbon dioxid and salts.

The skin, unlike the kidneys, is not set apart for the performance of one special function. It is not only an important excretory organ, but also an absorbing organ; it is likewise the principal seat of the sense of touch, and

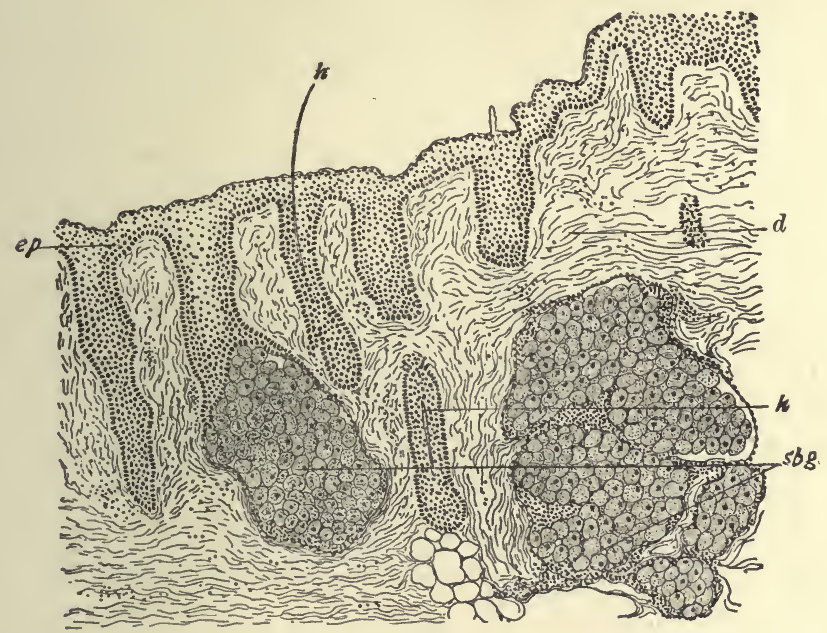

FIG. 97.- Vertical section of skin: $s b g$, Sebaceous glands; $e p$, epidermis ; $h$, hair ; $d$, derma (Fox).

also serves as a protective covering for the underlying tissues. The skin, like the mucous membrane, consists of two distinct layers - an epithelial covering and a connective-tissue basis. The epithelium is stratified, and is called the epidermis or scarf-skin; the connective-tissue layer is called the derma (true skin) or corium.

The epidermis is composed of layers of cells, the 
deeper of which are soft and protoplasmic, while the superficial layers are hard and horny. Between the two layers is a fairly distinct line of granular-looking cells, the granules in which have been thought to form the horny matter in the superficial cells. In the colored races the single layer of elongated cells next to the corium contains pigment-granules.

The epidermis, which throughout the body is of horny consistence, but so thin as readily to admit of flexibility, is on the ends of the fingers replaced by a thicker and more permanent texture of horny substance, called the nails (clavus).

In the palms of the hands and on the soles of the feet the skin is thickened and more firm, according as the parts are exposed to friction or wear.

The outer surface of the skin is covered by numerous small eminences or papilla, which are the terminations of the nerves and vessels, each papilla containing a nerve and one or more vessels.

The corium, as has been said, possesses great sensibility, and is commonly regarded as the starting-point of the nervous system to palpable feeling. It is, therefore, very largely supplied with nerves, and is also well furnished with blood.

The outer layer, or epidermis, possesses no sensibility, and yet, being extremely thin and delicate, it offers but little resistance to the sense of touch. It serves chiefly as a covering or shield for the under parts, to prevent irritation, corrosion, and the absorption of pernicious substances into the circulation.

The Sudorific and Sebaceous Glands.-The skin is furnished with numerous small glands termed secretory organs.

The sudorific glands secrete sweat or perspirable fluid from the circulation. Their function is of great import- 
ance, and serves, in the first place, to remove the excremental or exhausted material from the body; and, secondly, to regulate the temperature by means of evaporation of the fluid thus thrown out. For example, when the body has been exposed to heat or when exercise has been indulged in, perspiration is abundant, so that by its evaporation the heat may be dissipated.

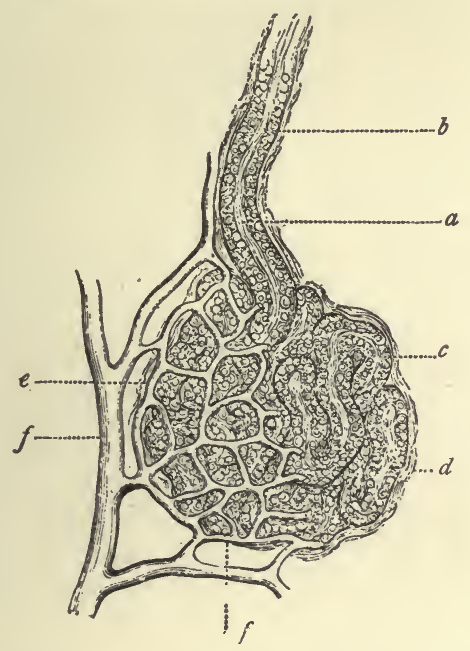

FIG. 98.-A normal sweat-gland, highly magnified: $a$, Sweat-coil, with secreting epithelial cells; $b$, sweat-duct ; $c$, lumen of duct; $d$, connectivetissue capsule; $e$ and $f$, arterial trunk and capillaries supplying the gland (after Neumann).

These little sweat or sudorific glands have a spiral outlet upon the surface, but their entire arrangement is so delicate and minute that they cannot be viewed by the unaided eye. In the illustration (Fig. 98) the parts showing the sudorific glands and the spiral outlet, passing through the different layers of skin, have been highly magnified.

The skin is also furnished with other glandular organs, 
the sebaceous glands which are situated in the armpits, and in the face, as about the nose and ears. These fur-

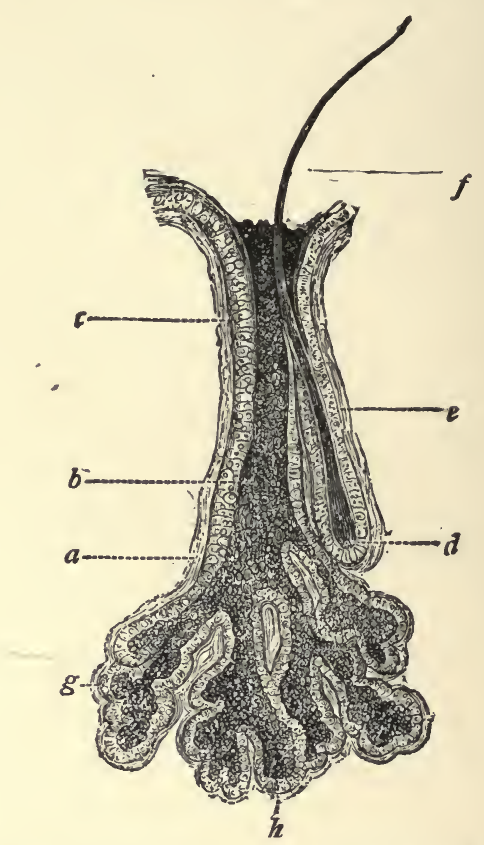

FIG. 99.-A normal sebaceous gland in connection with a lanugo hair; greatly magnified: $a$, Connective-tissue capsule; $b$, fatty secretion; $c, h$, fat-secreting cells; $d$, root of a lanugo hair; $e$, hair-sac $; f$, hair-shaft; $g$, acini of sebaceous gland (after Neumann).

nish an oily secretion to the skin in all parts, which keeps it soft and pliable.

\section{THE APPENDAGES OF THE SKIN}

The appendages of the skin are the hair and nails.

The hair is a peculiar modification of the epidermis, and consists essentially of the same structure as that membrane. It consists of a root, a shaft, and a point. The 
root presents a bulbous enlargement at its extremity; the shaft consists of a central part or meduilla.

Hairs are found on almost every part of the human body, but are most numerous on the scalp, and, in the

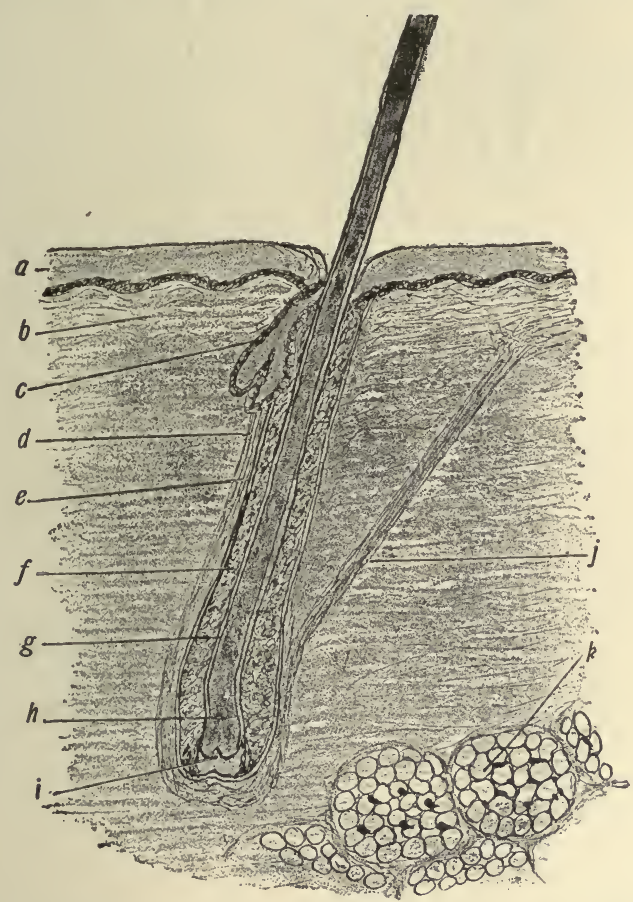

FIG. 100.-Skin and longitudinal section of hair: $a$, Epidermis; $b$, corium; $c$, sebaceous gland; $d$, fibrous root-sheath; $e$, glassy membrane; $f$, outer root-sheath; $g$, inner root-sheath; $h$, expanded bulbous end of hair ; $i$, papilla of hair; $j$, arrector pili ; $k$, adipose tissue (Leroy).

male, on the face, where, in the beard, they are thick and coarse in character. At puberty hair appears on the pubis and in the armpits.

The nails are flattened, elastic structures of a horny texture, situated upon the dorsal surface of the terminal 
phalanges of the fingers and toes. The nail has a firm attachment to the cutis, and the part beneath the body and root is called the matrix, from which successive growth
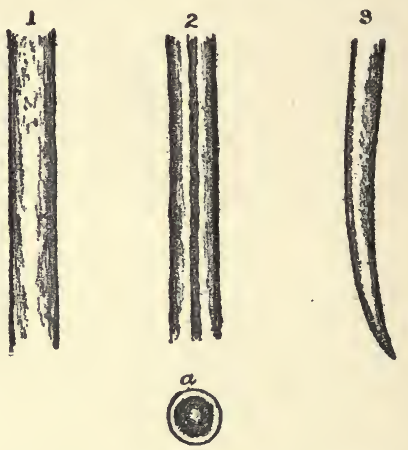

F1G. Ior.-Human hair: I, The hair of a child; 2 , hair of an adult; 3 , pointed extremity of the hair of the eyebrow ; $a$, transverse section of the hair, showing the cortical and medullary portions, and air-cells in the center of the cylinder (Chapman).

of the nail is produced, with the accumulation of the cells therein.

The chemic composition of the nails is the same as that of the epidermis, with a larger proportion of sulphur and carbon.

\section{REVIEW QUESTIONS}

What do you understand by metabolism?

What is known by elimination?

What are the waste products.?

What constitutes the excretory system?

How many kidneys are there?

What is the function of the kidneys?

Give the location of the kidneys.

What is their average weight?

What are the Malpighian bodies?

Describe the structure of the kidneys.

Describe the uriniferous tubules.

Describe the ureters. 
Describe the bladder.

Describe the urethra.

What is micturition?

Describe the characteristics of normal urine.

What is the specific gravity? Reaction?

Upon what is the color dependent?

What are the chief constituents of urine?

In what abnormal conditions do we find albumin, sugar, pus, uric acid?

What is the normal amount secreted in twenty-four hours for an adult in health?

How may the specific gravity vary, and under what conditions?

Describe the suprarenal capsule.

What are the sudorific glands? What is their function?

Describe sebaceous glands.

What important function do they possess?

How does the skin act as an excretory organ? Upon what is it dependent?

How many layers are there in the skin?

What are the appendages of the skin? 


\section{CHA P T E R V I}

\section{THE NERVOUS SYSTEM}

THE nervous system is concerned chiefly with the functions of sensation, motion, and volition. It is through this system that all actions, voluntary and involuntary, are controlled. It is made up of the cerebrospinal center, and the numerous slender or thread-like prolongations that emanate from the center, called nerves, and which are distributed to every tissue and part of the body, excepting the extremities of the nails, the hair, and adipose tissue. The substance of the brain, spinal cord, and nerves is very similar. It is of soft or pulpy consistence, and of a whitish or cineritious color.

The Brain.-The brain in the human subject is large, and, with its membranes and vessels, occupies the cavity of the skull. It consists of three portions-the cerebrum, or anterior and larger portion, the cerebellum, which is about one-seventh the size of the cerebrum, and is situated in the back and lower portion of the skull, and the medulla oblongata, situated below the cerebellum. The brain is entirely surrounded by membranes - the dura mater, the arachnoid, and the pia mater.

The dura mater is a firm membrane, which lines the cranial cavity and acting as the periosteum of the cranial bones. It forms septa-the falx cerebri, the falx cerebelli, and the tentorium-which form partitions between the different parts of the brain.

The arachnoid is a serous membrane that invests the brain and spinal cord. Like other serous membranes, it is a closed sac. 
The pia mater is a very vascular membrane. It invests nearly the entire surface of the brain, and dips in between its convolutions. It is the nutrient membrane of the brain, and receives all the blood from the carotid and vertebral arteries.

The Cerebrum. - The cerebrum is divided into two hemispheres by a cleft or fissure. In the fissure we find a circular or arched projection of the dura mater, called the falx cerebri. The object of this construction is to render support to this large pulpy mass. The upper surface of the cerebrum is marked by many undulations or convolutions, and the under surface admits of three divisions in each hemisphere - the anterior, the middle, and the posterior divisions.

When the brain is cut through its upper part horizontally, and the part removed so as to expose the cut surface, it will be discovered that the substance of this organ is of two distinct colors. On its outer, surface the brain, for a small depth, is of an ashen-gray or cineritious color, while the central portion is white. This darker portion is called its cortical or cineritious portion; in its outlines it follows the convolutions of the brain. The light-colored portion is called the medullary portion. The two hemispheres of the cerebrum are connected by a dense layer of transverse fibers, called the corpus callosum.

The Cerebellum.-This portion of the brain is situated in the occipital or back part of the skull. Like the substance of the brain, the substance of this portion is also of two colors. When the cerebellum is cut vertically, the white matter has a beautiful appearance, resembling the trunk and branches of a tree, and known as the "arbor vitce." The main stem of this structure contains a collection of gray matter, the corpus dentatum.

The Medulla Oblongata.-By referring to the illustration (Fig. IO2) you will see, at the base of the brain, the 
commencement of the spinal cord, here called the medulla oblongata. It is an elongated structure, consisting of three divisions or pairs of bodies, called the corpus pyramidale,

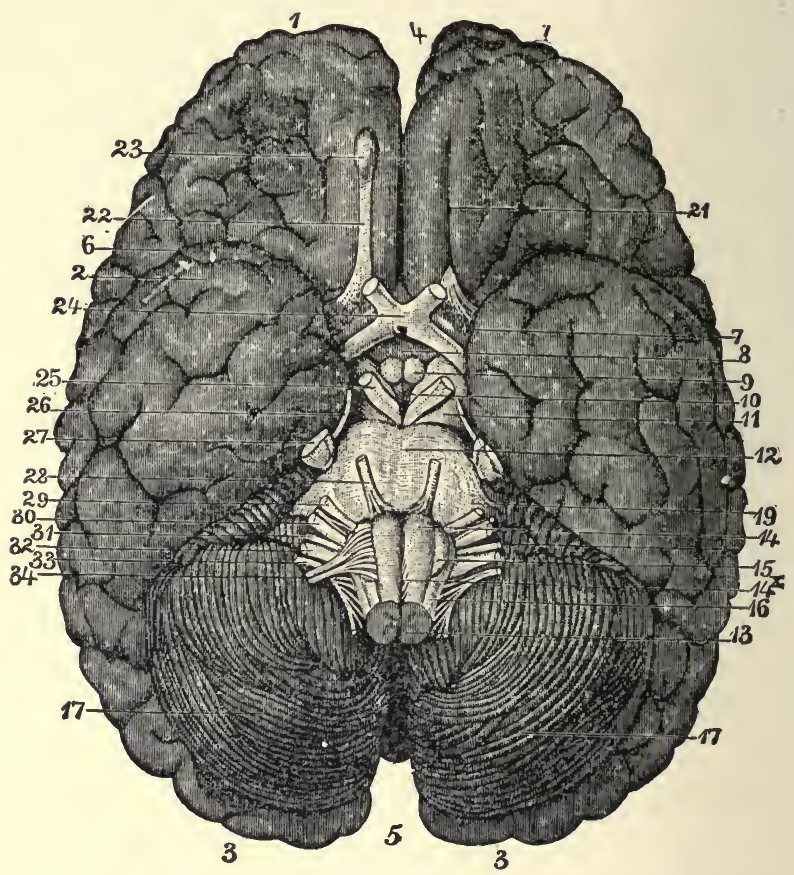

FrG. I02.-Base of brain: I, 2,3, Cerebrum ; 4 and 5, longitudinal fissure ; 6 , fissure of Sylvius; 7 , anterior perforated spaces; 8 , infundibulum; 9 , corpora albicantia; Io, posterior perforated space; II, crura cerebri; I2, pons Varolii ; I3, junction of spinal cord and medulla oblongata; I4, anterior pyramid; $14^{\mathrm{x}}$, decussation of anterior pyramid; 15 , olivary body ; I6, restiform body; I7, cerebellum ; I9, crura cerebelli ; 21 , olfactory sulcus; 22 , olfactory tract; 23 , olfactory bulbs; 24 , optic commissure; 25 , motor oculi nerve; 26 , patheticus nerve; 27 , trigeminus nerve; 28 , abducens nerve; 29 , facial nerve; 30 , auditory nerve; $3 \mathbf{I}$, glosso-pharyngeal nerve; 32 , pneumogastric nerve; 33 , spinal accessory nerve; 34 , hypoglossal nerve.

corpus restiforme, and corpus olivare, united in a single bulb. The medulla oblongata, unlike the brain, is highly sensitive, and if even slightly punctured, convulsions ensue. 
Most of the important vital centers are located in this portion of the brain. At this point all the special nerves diverge, so that an injury to this part produces immediate disturbance in the location to which the nerve is distributed.

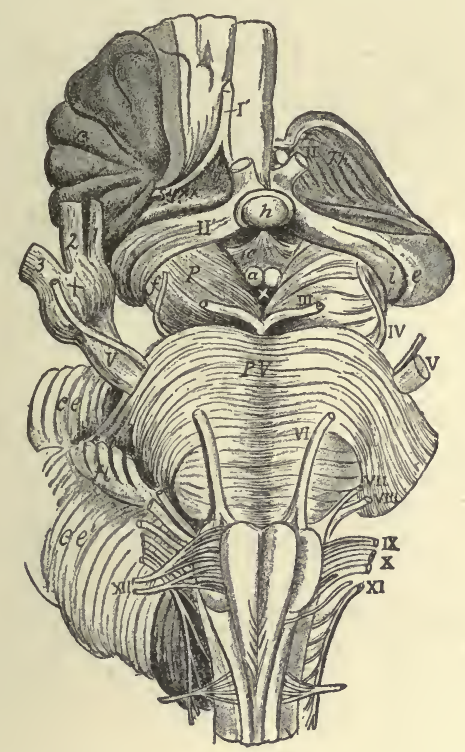

FIG. I03.-View, from below, of the connection of the principal nerves with the brain: $I^{\prime}$, The right olfactory tract; II, the left optic nerve; II', the right optic tract (the left tract is seen passing back into $i$ and $e$, the internal and external corpora geniculata); III, the left oculomotor nerve; IV, the trochlear; $\mathrm{V}, \mathrm{V}$, the large roots of the trifacial nerves $;++$, the lesser roots (the + of the right side is placed on the Gasserian ganglion); $I$, the ophthalmic ; 2 , the superior maxillary; and 3 , the inferior maxillary divisions; VI, the left abducens nerve; VII, VIII, the facial and auditory nerves; IX-XI, the glossopharyngeal, pneumogastric, and spinal accessory nerves; $\mathrm{XII}$, the right hypoglossal nerve; $\mathrm{C}_{1}$, the left suboccipital or first cervical nerve (Nancrede).

The Spinal Cord.-The spinal cord continues from the medulla oblongata downward through the spaces in the vertebral column afforded by the corresponding openings of the vertebræ. It runs throughout the entire length of 
the spinal canal, extending from the foramen magnum to the lower border of the first lumbar vertebra, where it terminates in slender filaments of gray substance. Its weight, when divested of all its membranes and nerves, is about $1 \frac{1}{2}$ ounces. It is usually about 16 or 17 inches in length. It varies in its diameter at different locations.

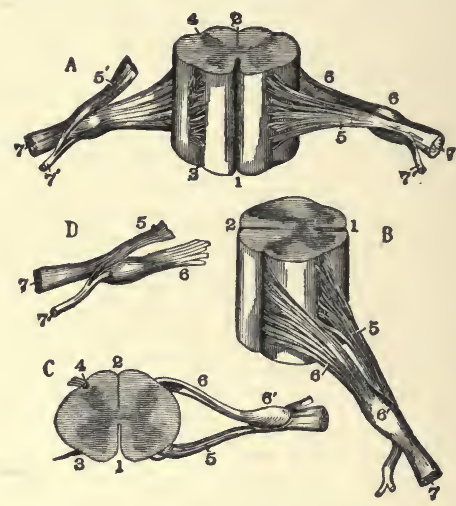

FIG. I04.-Different views of a portion of the spinal cord from the cervical region, with the roots of the nerves. In $A$ the anterior surface of the specimen is shown, the anterior nerve-root of $\mathrm{jts}$ right side being divided; in $B$ a view of the right side is given; in $C$ the upper surface is shown; in $D$ the nerve-roots and ganglion are shown from below: $\mathrm{I}$, the anterior median fissure; 2 , posterior median fissure; 3 , anterior lateral depression, over which the anterior nerve-roots are seen to spread; 4 , posterior lateral groove, into which the posterior roots are seen to sink; 5 , anterior roots passing the ganglion; $5^{\prime}$, in $A$, the anterior root divided; 6 , the posterior roots, the fibers of which pass into the ganglion, $6 ; 7$, the united or compound nerve; $7^{\prime}$, the posterior primary branch seen in $A$ and $D$ to be derived in part from the anterior and in part from the posterior root (Allen Thomson).

It is grooved, on both its anterior and its posterior surface, by a furrow that divides it in its entire length into two great nervous cords intimately united with each other. These lateral cords are each divided by furrows into three distinct sets of fibers or columns; namely, the anterior, lateral, and posterior columns. The anterior are the motor columns; the posterior, those of sensation; the 
lateral columns are divided in their function between motion and sensation.

The spinal cord terminates, at its lower extremity, in an oval tubercle, whence arise a number of nerves that go to the lower parts of the body. From the appearance these numerous nerves assume as they emerge from the spinal cord, they have collectively been denominated the "cauda equina," from a fancied resemblance to a horse's tail. The spinal cord, as well as the medulla oblongata, is enveloped in its entire length by the continuation of the three membranes of the brain. A more detailed description of the anatomic structure of the various portions of the nervous system will be given further on.

\section{THE NERVES}

The nerves are tubular cords, the substance of whose structure is similar to that of the brain and spinal cord. All nerves take their origin in the brain and the spinal cord, and extend in sets to every part of the body by different routes. They often unite in their course and form a plexus. There are 43 pairs of primary nerves arising from the nervous centers; these, according to their origin, are termed cranial, or encephalic, and spinal nerves. Twelve pairs originate within the skull and 3 I pairs pass from the spinal cord- 8 cervical, I 2 dorsal, 5 lumbar, 5 sacral, and I coccygeal.

Each nerve is composed of several filaments or cords, lying alongside one another, and surrounded by the neurilemma. Most of the cranial nerves have but a single root, but the spinal nerves arise by two roots-one from an anterior fasciculus of filaments and the other from a posterior; these are separated from each other by the ligamentum denticulatum. The two roots later join, however, to form one nerve.

The spinal nerves, as has just been stated, arise by 
two roots from the anterior and posterior columns of the spinal cord. The posterior roots are larger than the anterior. The anterior are the motor radicles, and the posterior, the sensory.

On each of the posterior roots, in the openings between the bones of the spinal column (intervertebral foramina),

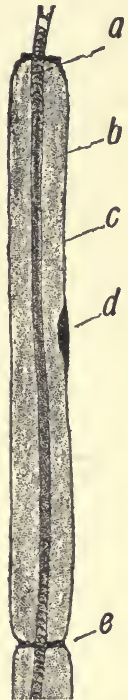

FIG. 105.-Longitudinal nervefiber (diagrammatic): $a$, Axiscylinder $; b$, medullary she ath $; c$, neurilemma ; $d$, nucleus; $\dot{e}$, node of Ranvier (Leroy).

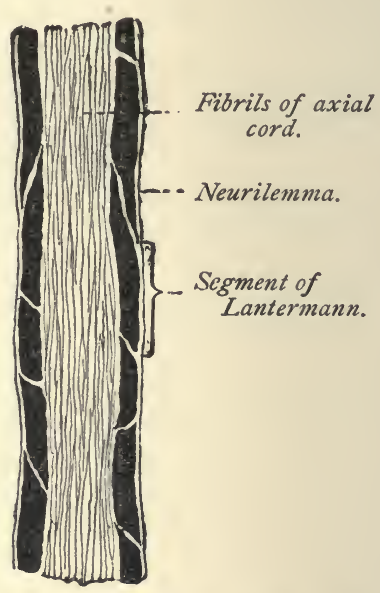

FIG. I06. - Longitudinal section through a nerve-fiber from the sciatic nerve of a frog $(\times 830)$ (Böhm and Davidoff).

a ganglion is formed, after which the anterior and posterior roots of the nerve unite and form the spinal nerve.

The ganglia may be considered as distinct centers, giving off branches in different directions: the superior, or ascending, to communicate with the ganglion above; the inferior, or descending, to communicate with the ganglion below; the external, to communicate with the sympathetic 
filaments. It is generally admitted that the nerves that emerge from the ganglia are larger than those that enter them-as if the ganglia imparted additional power to the nerve.

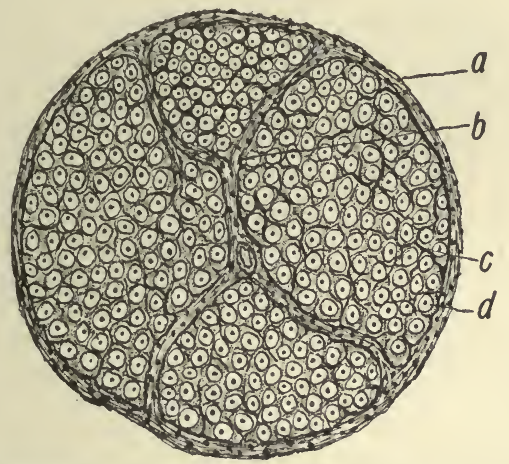

FIG. I07.-Transverse section of a nerve : $a$, Epineurium ; $b$, perineurium ; $c$, endoneurium; $d$, section of a single fiber (Leroy).

The branches of distribution accompany the arteries that supply the different organs and form communications around them; these are called plexuses, and take the name of the artery with which they are associated. Thus

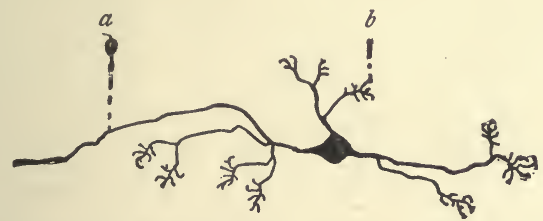

FIG. 108.-Nerve-cell with dendrites ending in claw-like telodendria : $a$, Neuraxis; $b$, telodendrion (Böhm and Davidoff).

we have the mesenteric plexus, the hepatic plexus, the splenic plexus, etc. All the internal organs of the head, neck, and trunk are supplied with branches from the sympathetic nerve, and some of them exclusively. This 
nerve, as well as the other important nerves of the body,

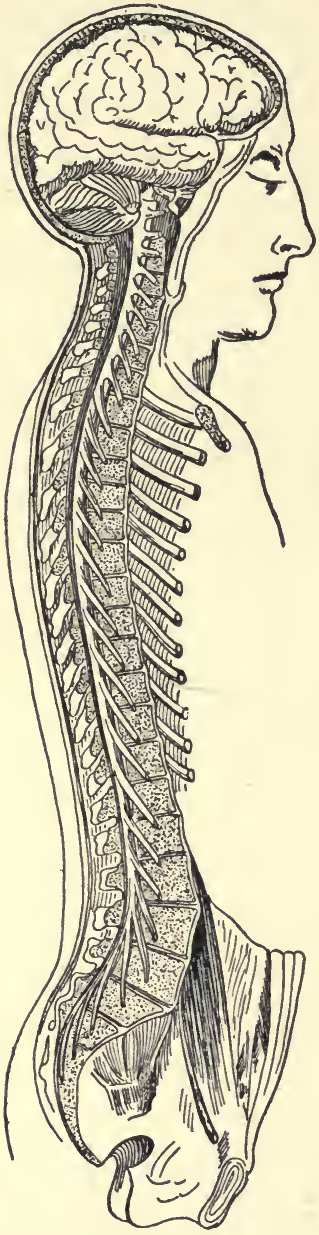

FIG. rog.-General view of the cerebrospinal nervous system (after Bourgery ; Schwalbe). Schwalbe). immediately affect the various
functions of life, such as the respiration, the digestion, and will be discussed elsewhere.

Functions of the Nervous System. - The cerebral hemispheres are the centers of the nervous system, through which are manifested all the phenomena of the mind; they are the centers in which impressions are registered and subsequently reproduced as ideas; they are the seat of intelligence, reason, and will.

However important a center the cerebrum may be for the exhibition of this highest form of nervous action, it is not directly essential for the continuance of life, for it exerts no control over those automatic reflex acts, known as respiration, circulation, etc., which regulate the functions of organic life.

The brain is not a sensitive organ, and may be lacerated, or portions may even be cut away without giving rise to much pain; such injuries, however, will immediately affect the mental faculties. The cerebellum, on the other hand, is in close relation with the functions of animal life, and any interference with it will immediately affect the various
the respiration, the digestion, and 
the circulation. The medulla oblongata appears to be the general receptacle of the impressions of the nerves of the special senses, and the spinal cord that of general sensation.

Functions of the Nerves.-Of the spinal nerves and of one of the cranial (trifacial), it may be stated that they have two roots-one for conveying motor and one for carrying sensory impulses. In other words, one set for the functions of motion and the other for those of sensation.

No difference has been discovered in the structure of the several kinds of nerves in any part of their course, and the functions they are designed to perform can only be learned by ascertaining the place of their origin. The nerves may be divided into five groups, according to their functions:

I. Nerves of Special Sensation.-There are the first, second, eighth, and ninth pair of cranial nerves, and are concerned with smelling, seeing, hearing, and tasting.

2. Nerves of General Sensation.-In those parts that require sensation for safety and the performance of their functions there is an abundant supply of sensitive nervous filaments. The nerves of sensation are distributed chiefly to the skin. Few filaments ramify in the mucous membranes and muscles. The head is supplied with sensation by the ophthalmic and superior maxillary divisions of the fifth cranial nerve; the remainder of the body by fibers from the mixed spinal nerves.

3. Nerves of Motion.-To these belong the third, the fourth, part of the fifth, the sixth, the seventh, the eleventh, and the twelfth pairs of cranial nerves. They are distributed to the muscular fibers, and, by their action, give rise to muscular contraction. (By referring to the illustration, Fig. II 4 , the several nerves that control the general system may be seen.) 
4. Mixed Nerves.-These contain both motor and sensory fibers. They are the inferior maxillary division of the fifth, the tenth pair of cranial nerves, and all of the spinal nerves.

5. The Sympathetic Nerve.-The sympathetic nerve, also called the trisplanchnic nerve, is in reality a system of nervous arrangement adapted particularly for the performance of the functions of organic life. It consists of a series of ganglia united by intermediate branches, and distributes its regular branches in the three great splanchnic cavities - those of the head, chest, and abdomen. In the trunk it lies mainly on the side of the spine (the student should carefully study the illustration, Fig. I I 4 , in order to become familiar with this important nerve) and communicates with all the spinal and several of the cranial nerves.

The sympathetic nerve serves to maintain vitality in all the important portions of the system. It exerts a controlling influence over the involuntary functions of digestion, absorption, secretion, circulation, and nutrition. Every portion of the body is, to a certain extent, under its influence, as filaments from this system of nerves accompany the blood-vessels throughout their course.

An important function of the sympathetic system is to form a communication of one part of the system with another, so that when one organ is affected, every other organ will act accordingly. If, for example, disease seizes the brain, the stomach, by its sympathetic connection, becomes aware of it; and since nourishment would augment the disease, the stomach refuses to receive food, and perhaps throws off what has already been taken. The loss of appetite in certain diseases is thus a kind provision of nature that prevents our taking food when it would be injurious; following this intimation, we, as a general rule, should abstain from food until the appetite returns. 
Having described briefly the several portions that go to make up the nervous system, we will now consider more fully the anatomy of the parts connected with these important structures.

\section{THE BRAIN}

This is that portion of the cerebrospinal center contained in the cavity of the cranium. It is divided into four principal parts: the medulla oblongata, pons Varolii, cerebrum, and cerebellum.

The average weight of the brain is about 49 ounces in the male and 44 ounces in the female; the heaviest male brain of which there is a record weighed $68 \frac{3}{8}$ ounces; the lightest, 34 ounces; the heaviest female brain weighed 56 ounces; the lightest, 30 ounces.

The brain is divided into fissures and lobes; the fissures divide the several parts of the brain substance, and in this manner the several lobes are marked out.

As has been stated elsewhere, the brain is surrounded by three membranes: the dura mater, which is a tough, fibrous membrane; the arachnoid, a serous membrane; and the pia mater, a vascular membrane.

The Dura Mater.-This is the first membrane to be encountered after the removal of the skull-cap. It derived its name from the fancied belief that it supplied all the membranes of the body. As has been said, it is a tough, inelastic, fibrous structure, forming the internal periosteum of the skull. It acts as a support for the lobes of the brain, and forms partitions for the falx cerebri, falx cerebelli, and tentorium cerebelli; it also provides sinuses for the veins that return the blood from the brain; further, it forms sheaths for the nerves as they leave the skull.

The Arachnoid Membrane.-This is the second membrane, and takes its name from its resemblance to a spider's 
web. It is a very delicate, serous membrane, and, like all other serous membranes, is a closed sac-one part, forming the parietal layer, lines the under surface; the other, or visceral layer, is reflected over the brain.

This membrane is perfectly smooth, and is sufficiently lubricated to prevent friction. It is in close connection with the pia mater. At the base of the brain it forms spaces, known as subarachnoidean spaces, which contain the cerebrospinal fluid; this fluid serves to protect the brain and nerve-centers from sudden shocks and concussions, such as blows, as well as loud noises. The base of the brain is supported by a bed of water. In fractures at the base of the skull or in the temporal region water will be seen to ooze through the ear and along the line of the fracture; this symptom is generally considered as proof of the presence of a fracture.

The Pia Mater.--This is the third membrane that invests the entire surface of the brain, and forms the velum interpositum and choroid plexuses of the third and fourth ventricles. It is extremely vascular, its bloodsupply being derived from the internal carotid and vertebral arteries, and forms a bed in which the blood-vessels divide and subdivide before they enter the brain. It dips in between the convolutions, as well as into the lateral ventricles, to supply blood to the interior of the brain substance.

The great mass of nervous matter termed the brain is, as we have said, divided into three great parts-the cerebrum, the cerebellum, and the medulla oblongata.

The cerebrum is of oval form: it is divided in the middle line, by the longitudinal fissure, into two parts, termed the right and the left hemisphere. The surface of the hemispheres is of tortuous character, and divided by furrows, or sulci, into convolutions. The furrows serve to accommodate the vessels in their course to the sinuses; they also 
contain fluid from the subarachnoid spaces. In old age, or in cases of disease in which the convolutions become shrunken, large quantities of water are present. The convolutions are folds of the brain substance, this folding serving to give greater extent to the circumference of the parts and also a greater area of gray matter. This is believed to be the location of the mental forces and of intelligence. The number of convolutions and their depth vary in different races.

The under surface of the brain resembles the upper; it presents the same convoluted appearance, and is made up of three lobes-the anterior, the middle, and the posterior. (See Fig. I02.)

The anterior lobe rests on the roof of the orbit, and is separated from the middle lobe by the fissure of Sylvius, which receives the lesser wing of the sphenoid bone. It contains the island of Reil. The middle lobe occupies the middle fossa of the base of the skull formed by the sphenoid and temporal bones. The posterior lobe rests upon the tentorium.

We will now examine the interior of the brain. In separating the hemispheres of the cerebrum in the longitudinal fissure we find what is termed the corpus callosum. In examining the brain we find it made up of two characteristic substances-the white and the gray matter. The gray matter is about $\frac{1}{8}$ inch in thickness, and invests the brain like a bark, hence it is termed the cortical substance. The furrows, as previously stated, vary in number and in depth, according to the intelligence of the individual. To the naked eye the cortical substance appears as one layer, but when examined under the microscope six layers are seen-three gray, alternating with three white. The central white tissue is called the medullary portion, and forms the mass of the brain substance. 
The corpus callosum is the transverse portion of the brain connecting the two hemispheres; it is about 4 inches long. It is composed of a body, a genu, and a splenium. It roofs in the lateral ventricles of the brain.

There are five ventricles in the brain. The two lateral are covered with the corpus callosum, and are formed by the folding backward of the cerebral lobes. They contain a quantity of a serous fluid, which, when it occurs in excess, as it sometimes does in children, constitutes the disease known as hydrocephalus. It has an anterior, a middle, and a posterior horn.

The third ventricle lies between the optic thalami and extends to the base of the brain.

The fourth ventricle is situated between the cerebellum and the posterior parts of the medulla oblongata and pons Varolii.

If a perpendicular section of the brain were made, the lateral ventricles, as well as the third, would be seen; the corpus callosum, and the fornix and velum interpositum below it, would also come into view.

The fornix is a layer of white matter, arranged in the form of an arch, beneath the corpus callosum.

The velum interpositum is a reflection from the pia mater, which penetrates the ventricles through the fissure beneath the posterior border of the corpus callosum. It is a vascular veil, and is rolled up in form of a fringe, which is called the choroid plexus. These plexuses are made up of minute arteries, and communicate with each other through the foramen of Monro. The foramen of Monro is not really a foramen, but simply a communication between the third and the two lateral ventricles. The venæ Galeni, two in number, return the blood through these parts and ventricles into the straight sinus.

The optic thalamus is an elevation upon the floor of the lateral ventricles immediately behind the corpus striatum. 
The corpus striatum is a layer of white and gray matter; the under part corresponds with the convolutions at the base of the brain, known as the island of Reil.

The tubercula, or corpora quadrigemina, are four rounded eminences, situated two on each side behind the pineal gland; they consist of white and gray matter; the gray matter of the anterior pair giving off the optic nerve, which pierces the ball of the eye; hence they are sometimes termed the optic lobes.

The cerebellum is situated in the occipital fossa, below the posterior lobes of the cerebrum. It is elliptic, its broad diameter being transverse. Upon transverse section it presents an appearance resembling the branches of a tree; hence, as has been stated elsewhere, it has been termed the arbor vitce, or "tree of life." It is considered to be more complex in its anatomic arrangement than any other part of the body.

The pons Varolii is situated at the base of the brain, just above the medulla oblongata, and rests upon the basilar surface of the occipital bone. It has a diameter of about $I$ inch. The basilar artery runs in a groove upon its inferior surface.

The medulla oblongata is that part of the central cerebrospinal axis that connects the cerebrum with the spinal cord, it is also connected with the cerebellum by the restiform bodies. It is one of the most important divisions of the nervous system, and contains the nervecenters for speech, deglutition, and respiration. Injury to this structure is, indeed, fatal.

The medulla oblongata is about $\mathrm{I} \frac{1}{4}$ inches long, and lies above the basilar groove of the occipital bone; it passes backward through the foramen magnum, at which level the spinal cord begins.

It is divided into anterior pyramids which are continuous with the spinal cord on the anterior of the same; 
they are pyramidal in form, and the motor fibers of each side decussate with one another. This is apparent from the fact that in conditions in which one side of the brain is affected, loss of power is manifested on the opposite side of the body. The decussation takes place only between the inner fibers of the pyramids, and also from the lateral columns of the cord.

The olivary bodies are situated on the outer side of the pyramids. The restiform bodies are on the outer side of and behind the olivary bodies. They diverge from each other and pass into the cerebellum.

The Arterial Supply to the Brain.-As was stated in a previous chapter, the circle of Willis is formed by the two internal carotids and the two vertebral arteries.

The internal carotid artery enters the skull through a canal in the petrous portion of the temporal bone; it becomes very tortuous, and passes by the side of the sphenoid bone, giving off the ophthalmic artery. It divides into the anterior and middle cerebral arteries, which supply the anterior and middle cerebral lobes of the brain. (This artery has been more fully considered in the chapter on the Circulatory System.)

The anterior cerebral artery distributes branches in all directions. It runs forward upon the lower surface of the brain, and then curves round in the front of the corpus callosum to pass backward along the dorsal surface of this structure. It is connected, soon after its origin, with the artery of the opposite side by a communicating branch. The middle cerebral artery runs outward along the fissure of Sylvius, distributing large branches to the anterior and middle lobes of the brain.

The vertebral artery, after winding backward along the arch of the atlas, enters the skull through the foramen magnum, and unites with its fellow at the lower border of the pons, to form the basilar artery; it then divides at the 
middle of the pons into the two posterior cerebral arteries, which supply the posterior cerebral lobes. At the pons the basilar gives off the auditory artery, which enters the internal auditory meatus together with the auditory nerve. It also gives off the superior and inferior cerebellar arteries for the supply of the upper and lower surface of the cerebellum, and eventually enters the circle of Willis.

The circle of Willis provides a free supply of blood from the several arteries of which it is formed-the anterior communicating in front; the two posterior communicating behind; the former running between the two anterior cerebral arteries, the latter from the posterior cerebral to the carotid. The tortuosity of the vessels before entering the brain provides a means to divert the excessive force of the blood in the small vessels; the four vessels that enter the skull break up and form minute branches, and it is for this reason that congestion of the brain is relieved only with difficulty and recovery therefrom is slow.

In considering the cerebral circulation, mention must be made of the important part played by the meningeal arteries.

The meningeal arteries ramify between the dura mater and the skull. Their most important branch is the middle meningeal, a branch of the internal maxillary artery, which enters the skull through the foramen spinosum, passes through a groove in the sphenoid bone and anterior angle of the parietal bones to the top of the skull, giving off branches in every direction. The arteries are accompanied by corresponding veins. The other branches are of less importance-the anterior is given off from the internal carotid within the cranial cavity; the posterior is a branch of the occipital, and enters the skull through the jugular foramen.

The sinuses of the brain are venous channels; they run in pairs and as single sinuses; there are five pairs 
and five single sinuses. It is a peculiarity of the cerebral circulation that the venous blood is returned through these canals or sinuses.

The cerebral sinuses are formed by a separation of the dura mater into layers and are unyielding structures. They are lined by the same smooth membrane that lines the veins.

As has been said, there are fifteen sinuses; their names are given below, and their arrangement should be studied.

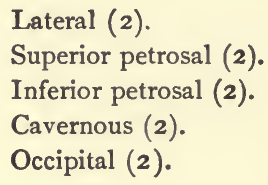

Circular.

Superior longitudinal. Inferior longitudinal.

Transverse.

Straight.

All these eventually discharge their blood into the internal jugular veins.

The spinal system of veins is considered together with the sinuses, as there is an extensive communication of vessels along the spinal column, their structure resembling that of a ladder; these all discharge through the intervertebral foramina into the several regions of the spine as follows: In the cervical region, into the vertebral veins; in the dorsal region, into the intercostal veins; in the lumbar region, into the lumbar veins. These veins are not provided with valves, which accounts for the serious conditions that result from injuries and inflammation of the spine.

\section{THE SPINAL CORD}

The spinal cord does not occupy the whole area of the spinal canal; the space is filled up with a reddish, fatty substance, and with the associated ramification of veins.

The Membranes of the Cord.-The dura mater does not adhere to the vertebra, therefore it is not called the internal periosteum, as in the skull; if this were the case, 
the adhesions would interfere with the movements of the vertebra upon each other. The dura mater sends off branches over each of the spinal nerves. The arachnoid membrane is continuous with that of the brain. Its spaces contain a watery fluid in which the cord may float.

The pia mater immediately invests the cord, and serves to support it; it plays a different rôle here, therefore, than it does in the brain. It is less vascular and

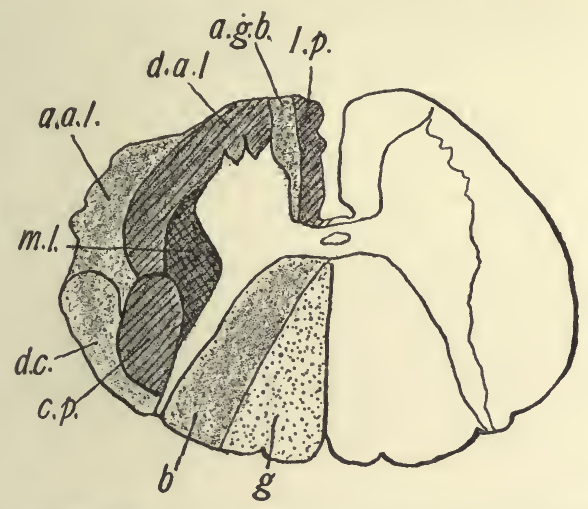

FIG. Iro.--Spinal cord, cervical region (diagrammatic), showing secondary tracts of white matter, the dark area representing descending fibers, dotted area ascending fibers, cross-lined area mixed fibers, and clear area gray matter: l.p., Direct pyramidal tract; $a . g . b .$, antericr ground-bundle; $d . c$., direct cerebellar tract ; a.a.l., ascending anterolateral or Gowers' tract ; c.p., crossed pyramidal tract; d.a.l., descending anterolateral tract; $m . l$., mixed lateral tract; $g$, column of Goll; $b$, column of Burdach (Leroy).

more fibrous in structure than the pia mater of the brain. It is closely adherent to the cord, forming what is called the neurilemma. The pia mater sends off ligaments from each side to steady the spinal cord; these are called the ligamenta dentata; they are eighteen to twenty in number on each side, and lie between the anterior and posterior roots of the spinal nerves.

The spinal cord is that part of the cerebrospinal axis contained in the vertebral canal. It is a continuation of 
the medulla oblongata, and runs to the upper border of the first or the second lumbar vertebra, where it divides into a bundle of nerves, called the cauda equina, which supply the lower limbs.

The cord is cylindric in form, and slightly flattened from before backward: On its anterior and posterior surfaces it presents fissures; laterally it is provided with two grooves on each side, from which the spinal nerves

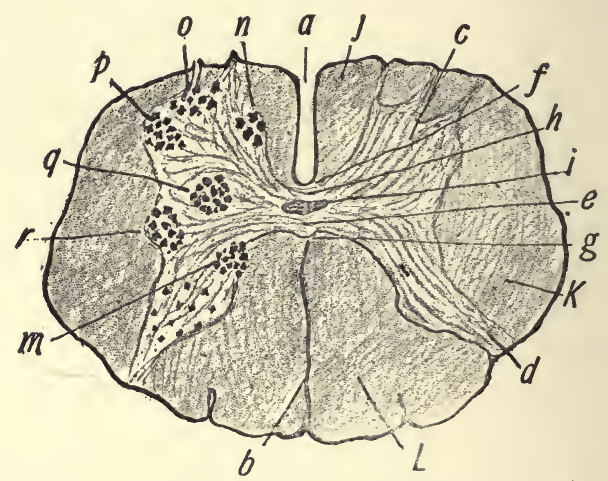

FIG. III.-Lumbar section of spinal cord, showing main tracts of white substance and location of principal groups of nerve cells in gray matter $: \cdot a$, Anterior median fissure; $b$, posterior median fissure; $c$, anterior horn of gray matter; $d$, posterior horn of gray matter ; $e$, central canal; $f$, anterior white commissure; $g$, posterior white commissure; $h, i$, anterior and posterior gray commissures; $j$, anterior median column; $K$, lateral column; $L$, posterior column; $m$, column of Clarke; $n$, inner group of nerve cells ; $o$, anterior group; $p$, anterolateral group; $q$, posterolateral group ; $r$, lateral horn (Leroy).

emerge. It is divided into three columns-anterior, posterior, and lateral; the fibers of the anterior are motor, the posterior sensitive, while those of the lateral are of both varieties.

In making a transverse section of the cord it will be observed (see Fig. I I ) that the interior contains gray matter, which resembles two crescents placed one in each half, and connected across the center by a band called the 
gray commissure. Each crescentic mass has an anterior and a posterior horn. The posterior horns are long and narrow, and are connected with the posterior roots of the spinal nerves. The anterior horns are shorter and thicker, and are directed forward toward the anterior roots of the nerves, but do not reach the surface.

The spinal nerves will be subsequently considered.

\section{THE NERVES OF THE GENERAL SYSTEM}

We will now discuss the several nerves that control the general system. Of these, the first to be considered are the cranial.

The Cranial Nerves.-The first, or olfactory, is the nerve of smell. It arises from the olfactory bulb and terminates in the olfactory tract, which has three rootsinner, middle, and outer. The olfactory nerves, coming from the olfactory bulb, are divisible into three groups: the inner supplies the mucous membrane of the septum of the nose; the middle supplies the roof of the nose; the outer supplies the turbinated bones. The common sensibility of the mucous membrane of the nose is derived from the fifth nerve.

The second, or optic, nerve is the nerve of sight. It arises from the anterior pair of the corpora quadrigemina and optic thalami. It rests on the sphenoid bone, then passes through the optic foramen to terminate in the retina. This nerve decussates at the middle of the commissure, a fact for which there seems to be no definite reason.

The third pair, or the motor oculi, apparently arises from the inner surface of the crus cerebri, immediately in front of the pons; it passes through the sphenoid fissure and supplies all the muscles of the eye, except the superior oblique and the external rectus.

The fourth nerve, or pathetic, arises from the valve of 
Vieussens, enters the orbit through the sphenoid fissure, and supplies the superior oblique muscle of the eye.

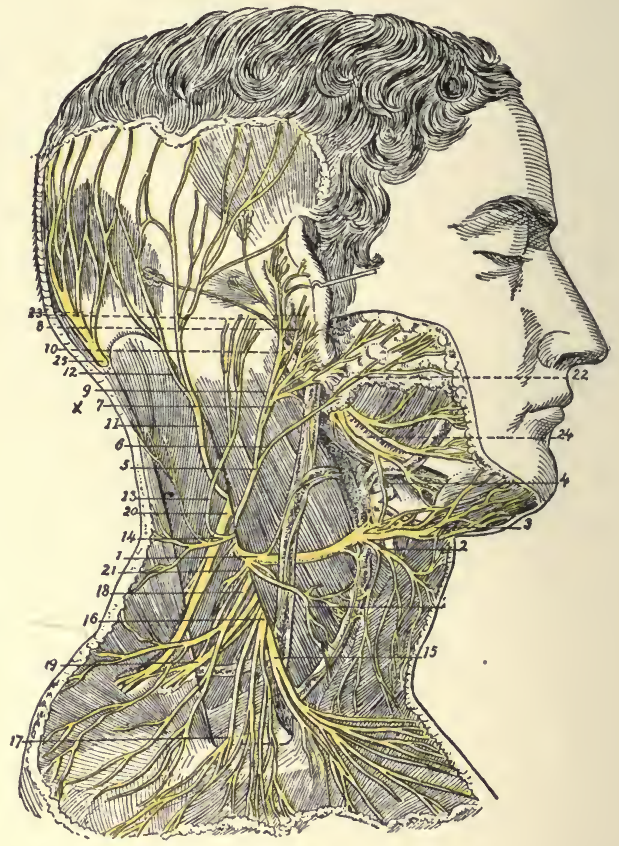

FIG. II2.-Superficial branches of cervical plexus: I, Superficial cervical nerve; 2 , its inferior branch; 3 , its superior branch; 4 , its union with facial; 5, great auricular nerve; 6 , one of its facial branches; 7 , its branch to lobule; 8 , twig which pierces the auricle; 9 , branch to deep surface of pinna; IO, its union with posterior auricular of the facial; II, small occipital nerve; I2, its branches; I3, a mastoid branch; I4, twigs from this to back of neck; I5, inner; I6, I7, middle; I8, outer branches of supraclavicular nerves; I9, branch of cervical nerves passing into trapezius muscle; 20, spinal accessory distributed to same, and receiving a uniting branch from the cervical nerves; 2 , branch to levator scapulæ; 22, trunk of the facial nerve; 23 , its posterior auricular branch; 24 , its cervical branch; 25 , great occipital nerve.

The fifth nerve, called the trifacial, is the great sensory nerve of the head and face and the motor nerve of the muscles of mastication. It is a nerve of general sensation 
and motion, and arises by two roots from the floor of the fourth ventricle of the brain. The sensory root has developed upon it one of the most important ganglia of the head-the Gasserian. This nerve gives off three branches - the ophthalmic division of the fifth, the superior maxillary, and the inferior maxillary. (See Fig. I 12.)

The ophthalmic division again divides, giving off the frontal, the lacrimal, and the nasal branches. The ophthalmic is a sensory nerve, and supplies the eyeball, ciliary muscles, iris, lacrimal gland, nasal and ocular mucous membrane, skin, and the muscles of the eyebrow, forehead, and nose.

The superior maxillary is also a sensory nerve, and is distributed to the temple, cheek, lower eyelid, nose, upper lip, teeth, the palate, and the pharynx. This branch divides again into the several nerves that supply the various parts, as follows: Superior dental, infra-orbital, meningeal, two sphenopalatine, and orbital. The superior dental divides into the anterior, middle, and posterior dental. The infra-orbital divides into the palpebral, nasal, and labial.

The inferior maxillary division of the fifth nerve is subdivided into the meningeal, masseteric, three temporal, buccal, two pterygoids, lingual, auriculotemporal, and inferior dental, which last subdivides into the dental, mylohyoid, incisive, and mental. The inferior maxillary is a nerve of common sensation and motion. It supplies the muscles of mastication, gums, temples, external ear, teeth, lower lip, tongue, maxillary ganglia, and lower part of the face.

The sixth nerve, or the abducens, arises from the medulla oblongata close to the poris, passes through the sphenoid fissure, and supplies the external rectus of the eye.

The seventh nerve, or facial nerve, arises as two por- 
tions. It is the motor nerve of the muscles of the face. It takes its origin in the pons Varolii and lateral columns of the medulla, and has its deep origin in the floor of the fourth ventricle. Its branches are the tympanic, chorda tympani, posterior auricular, digastric, stylohyoid, temporofacial (which subdivides into temporal, malar, infraorbital), cervicofacial (which subdivides into buccal, supramaxillary, and inframaxillary). The facial communicates with the auditory nerve; with Meckel's ganglion by the large petrosal; with the optic ganglion by the small petrosal; with the sympathetic on the middle meningeal by the external petrosal nerve; with the pneumogastric, glossopharyngeal, carotid plexus, åuricularis magnus, auriculotemporal, and with the three divisions of the fifth nerve. The importance of this nerve is apparent from its many communications, and it can readily be seen that injury to a part will interfere with the several relations of this nerve structure.

The eighth, or auditory, is the nerve of hearing. It begins by two roots, arising from the medulla oblongata; it is distributed to the internal ear by two branches- the vestibular, to the vestibule, and the cochlear, to the cochlea.

The ninth, or glossopharyngeal, is a nerve of common sensation, and also the nerve for the special sense of taste. It arises from the medulla oblongata, behind the olivary bodies, the deeper origin being from the floor of the fourth ventricle. This nerve emerges through the jugular foramen, and presents two enlargements or ganglia-the jugular and the petrosal. It is distributed to the muscles of the pharynx, mucous membranes of the pharynx, fauces, tonsils, tongue, and middle ear; for that purpose it gives off the following branches, which are distributed to the various parts indicated by their names: Tympanic, carotid, pharyngeal, muscular, tonsillar, and lingual.

The tenth, the pneumogastric, called the vagus nerve, 
has many points of interest; it gives off a great many nerves to important structures, and is both motor and

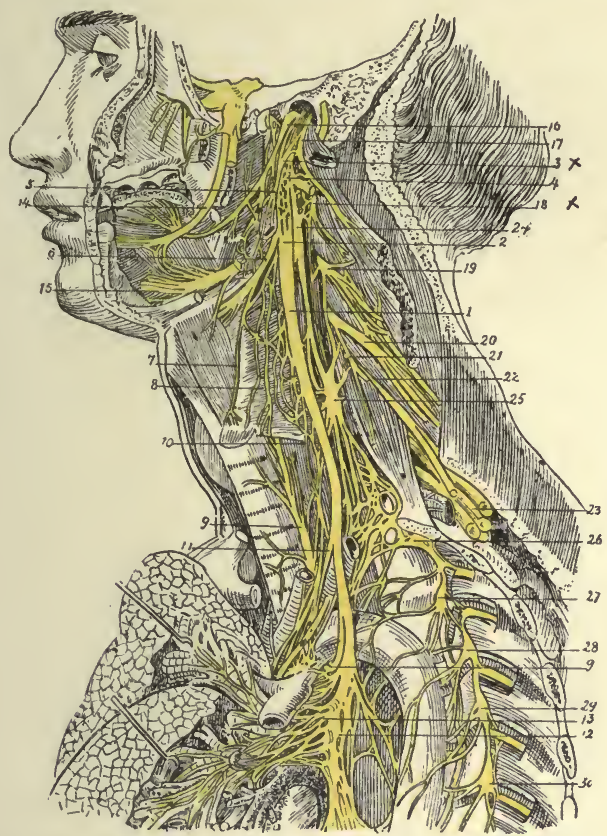

FIG. II3.-Distribution and connection of pneumogastric nerve of left side in neck and upper part of thorax: I, Pneumognstric nerve; 2, ganglion of its trunk; 3 , accessory part of spinal accessory ; 4 , union of pneumogastric with hypoglossal; 5 , pharyngeal branch of pneumogastric; 6 , superior laryngeal ; 7 , external laryngeal ; 8 , communication of external laryngeal with superior cardiac branch of sympathetic; 9 , inferior or recurrent laryngeal; Io, superior, and II, inferior, cervical cardi ic branches; I2, I3, posterior pulmonary plexus; I4, lingual branch of inferior nıaxillary; I5, distal part of hypoglossal nerve; 16, glossopharyngeal nerve; 17 , spinal accessory nerve; 18 , second cervical nerve; 19 , third $; 20$, fourth; 21 , origin of phrenic nerve; 22, 23, fifth, sixth, seventh, and eighth cervical nerves, forming with the first dorsal the brachial plexus; 24, superior cervical ganglion of sympathetic ; 25 , middle cervical ganglion ; 26 , inferior cervical gan glion; $27,28,29,30$, second, third, fourth and fifth dorsal ganglia.

sensory. It arises from the floor of the fourth ventricle, passes through the jugular foramen, and presents two gan- 
glia. It is distributed to the vocal cords, lungs, pharynx, esophagus, stomach, and heart. It gives off the following branches: Meningeal, auricular, pharyngeal, superior laryngeal, recurrent laryngeal, cervical cardiac, thoracic cardiac, anterior pulmonary, posterior pulmonary, esophageal, gastric, and abdominal plexuses. (See Fig. I I3.)

The eleventh, or spinal accessory, is a motor nerve, having its origin in the medulla oblongata, and making its exit through the foramen magnum; it is distributed to the muscles of the neck, pharynx, and palate, and is said to be a constrictor nerve of the heart.

The twelfth, or hypoglossal, is a motor nerve that supplies the tongue. It arises from the floor of the fourth ventricle, and makes its exit through the anterior condyloid foramen. It gives off the meningeal, muscular, and vascular branches. It supplies the tongue and the internal muscles of the throat, and communicates with the second and third cervical and sympathetic nerves.

The spinal nerves arise from the spinal cord. There are 3 I pairs of these nerves -8 cervical, I 2 dorsal, 5 lumbar, 5 sacral, I coccygeal. The function of these nerves has already been described. The following notes are taken from the researches of Blandin. ${ }^{1}$ He says, regarding the relative size of the anterior and posterior roots of the several regions of the spine:

The posterior roots are to the anterior in the cervical region . . . 2 : I The posterior roots are to the anterior in the dorsal region . . . I : I The posterior roots are to the anterior in the lumbar and

sacral regions . . . . . . . . . . . . . . I I $\frac{1}{2}:$ I

This writer states further that this relation quite accords with the great delicacy of the sense of touch in the upper extremities.

Each spinal nerve, as has been stated, arises by two ${ }^{1}$ Anat. descript., 1838 , t. ii., p. 648. 
roots - an anterior or motor root, and a posterior or sensory root. We will now consider the several spinal nerves individually.

The brachial plexus, sometimes called the axillary, is made up of the anterior divisions of the four lower cervical and first dorsal nerves.

The nerves forming the plexus communicate as follows: The fifth and sixth nerves unite near their exit from the spine to form a common trunk; the seventh nerve joins this trunk near the outer border of the middle scalenus muscle, and the three nerves thus form one large single cord. The eighth cervical and the first dorsal nerves form a common trunk. Thus two large trunks are formed, the upper one by the union of the fifth, sixth, and seventh cervicals; and the lower by the eighth cervical and the first dorsal. These two trunks accompany the subclavian artery to the axilla, lying upon the outer side, the trunk formed by the union of the last cervical and the first dorsal being nearest the vessel. Opposite the clavicle, and sometimes in the axilla, each of these cords gives off a fasciculus, which, uniting, form a third trunk, so that in the center of the axilla three cords are formed. The brachial plexus communicates with the cervical plexus by a branch from the fourth to the fifth nerve, and with the phrenic by a branch from the fifth cervical, which joins that nerve on the scalenus muscle. It is through this latter addition that in cardiac pains the sensation is felt in the elbow. The three cords of the brachial plexus are placed one on the inner side of the axillary artery, one behind, and the third on the outer side.

The plexus is broad, becoming narrower as it passes down the arm, but at the axilla, where it divides into terminal branches, it is larger. This plexus communicates with the cervical plexus by a branch from the fourth nerve, and also receives branches from the two inferior 
cervical ganglia of the sympathetic. At the neck it gives off the following branches, which are distributed to the several regions that each name designates.

Thoracic region.

Anterior thoracic.

Posterior thoracic.
Scapular region.

Superior muscular.

Suprascapular.

Subscapular:

Brachial region.

Musculocutaneous.

Median.

Internal cutaneous.
Circumflex.

Ulnar.

Lesser internal cutaneous.

Musculospiral.

The Dorsal Nerves.- These are twelve in number, and correspond to the dorsal vertebræ; they supply the several muscles of the chest and side.

The intercostals are formed from the dorsal and supply the intercostal spaces, pleura, mammary vessels, the muscles of the chest and side of the throat; the six lower supply the internal oblique and transversalis muscles, and continue to the sheath of the rectus; after supplying this muscle they go on to the linea alba, and, diverging, form the anterior cutaneous nerves of the abdomen.

The lumbar nerves are five in number on each side. The anterior branches of the four upper nerves anastomose to form the lumbar plexus. The plexus sends off the following branches:

The Iliohypogastric Branch.-This proceeds from the first lumbar nerve, and supplies the muscles of the hip and abdomen; it is distributed to the iliac region from the iliac branch; to the internal abdominal ring from the hypogastric branch.

The ilio-inguinal nerve arises from the first lumbar, and supplies the external abdominal ring, the pudenda, sper- 
matic cord, scrotum, and the integument of the upper thigh.

The genitocrural nerve arises from the second lumbar, enters the muscles of the anterior part of the thigh, and descends to near Poupart's ligament, where it divides into the genital and crural branches. The genital gives off branches to the internal abdominal ring and to the genital organs of the female; at the internal abdominal ring it sends off branches to the internal oblique and transversalis muscles, and is lost in the integument of the groin. The crural branch, the most external, descends along the outer border of the external iliac artery, and enters the sheath of the femoral artery; it is distributed to the upper as well as the anterior aspect of the thigh, communicating with the middle cutaneous nerve.

The external cutaneous nerve arises from the second lumbar nerve, or from a loop between it and the third; it passes into the thigh, beneath Poupart's ligament, where it divides into two branches - the posterior and the anterior, the posterior furnishing branches to the tensor vaginæ femoris muscle and posterior part of the thigh; the anterior divides into several branches, which are distributed to the outer border of the thigh and knee.

The obturator is formed from the third and fourth lumbar nerves. It makes a distribution to the obturator muscles, femoral and popliteal arteries, knee-joint, hip, and integuments of the upper and inner part of the thigh. At its origin it courses along the common iliac until it reaches the pelvic brim, where it passes through the obturator foramen and joins, in its course, the obturator artery; having escaped from the pelvis, it divides into anterior, posterior, and accessory branches, which supply the several muscular structures of the lower extremity.

The anterior crural nerve, sometimes called the femoral nerve, is the largest branch of the lumbar plexus; it is 
formed by the union of the second, third, and fourth lumbar nerves. It is distributed to the several muscles of the thigh; at Poupart's ligament it separates, and is divided into an anterior and a posterior part, which give off the following branches, which supply the structures their names imply. Anterior division: Middle cutaneous, internal cutaneous, long saphenous. Posterior division: Muscular and articular.

The sacral nerves are five in number on each side; they pass through the sacral foramina, the last one running between the coccyx and sacrum; their function is to supply the several pelvic organs. The coccygeal nerve supplies the coccygeus muscle and unites with the fifth sacral.

All the anterior cords of the sacral nerves communicate with the sympathetic at the points where they leave the sacral canal.

The sacral plexus of nerves is composed of the lumbosacral and the anterior branches of the three upper and part of that of the fourth sacral nerves; it gives off the following branches: Visceral, muscular, superior gluteal, pudic, small sciatic, and great sciatic.

The great sciatic is a continuation of the main part of the sacral plexus, forming the largest nerve in the body. It supplies nearly the whole integument of the leg, the muscles of the back of the thigh, and those of the leg and foot. At the lower third of the thigh it divides into two branches, the internal and external popliteal nerves.

The internal popliteal nerve is a branch from the great sciatic. This nerve with its branches supplies the posterior part of the leg; it finally divides into the internal and external plantar which supply the foot.

The external popliteal is smaller than the internal; it supplies the posterior muscles of the leg, and divides into two branches-the anterior tibial and musculocutaneous. 
The anterior tibial supplies the tibial artery and the several muscles of the anterior part of the leg, and furnishes branches to the foot and the articulation of the tarsus and metatarsus. The musculocutaneous nerve passes down along the fibula, supplying the peroneal and extensor muscles of the foot; the internal branch supplies the adjacent parts of the foot and toes; the external supplies the toes and communicates with the external saphenous nerve.

\section{THE GREAT SYMPATHETIC NERVE}

This nerve is divided into two portions: the vertebral and the prevertebral.

The vertebral portion is divided into ganglia, which unite and form a cord running longitudinally; it passes alongside the vertebral column from the head to the coccyx, communicates with the spinal and cranial nerves, and distributes branches to the internal organs and viscera.

The prevertebral portion also comprises a number of ganglia that form plexuses in the head, chest, abdomen, and pelvis.

The sympathetic nerve, as has been previously stated, communicates with the cerebrospinal nerves immediately at their exit from the cranium and vertebral canal. (See Fig. II 4.)

The branches accompany the arteries that supply the several organs, and form plexuses that surround the blood-vessels; they take the name of the artery they accompany. All the parts of the head, neck, and trunk are supplied, some of them exclusively, by branches from the sympathetic; therefore it has been called the nerve of "organic life." Since it contains so great a number of ganglia, which is evinced from the disposition it has to communicate with others in its distribution, it has also 
been termed the ganglionic nerve. Its functions has been previously described.

The Ganglia.-There are four ganglia in the head: the ophthalmic, sphenopalatine (Meckel's), otic or Arnold's, and the submaxillary; three in the neck: superior,

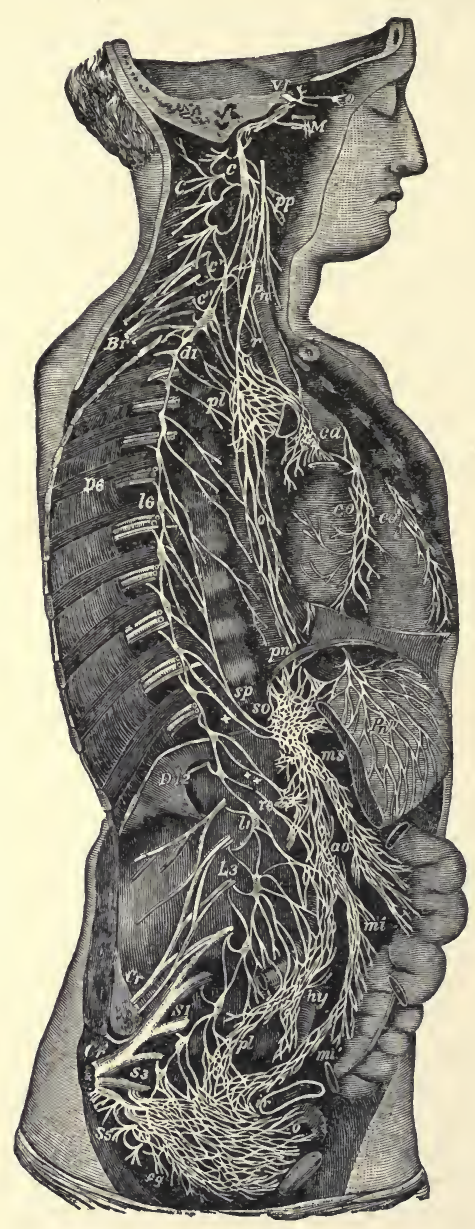

FIG. II4.-Diagrammatic view of the sympathetic cord of the right side, showing its connections with the principal cerebrospinal nerves and the main preaortic plexuses. (Reduced from Quain's Anatomy.)

Cerebrospinal Nerves: VI., A portion of the sixth cranial as it passes through the cavernous sinus, receiving two twigs from the carotid plexus of the sympathetic nerve; $O$, ophthalmic ganglion connected by a twig with the carotid plexus; $M$, connection of the sphenopalatine ganglion by the Vidian nerve with the carotid plexus; $C$, cervical plexus ; $B r$, brachial plexus; $D 6$, sixth intercostal nerve; $D$ I2, twelfth; $L_{3}$, third lumbar nerve; $S$ r, first sacral nerve; $S_{3}$, third; $S_{5}$, fifth ; $\mathrm{Cr}$, anterior crural nerve; $\mathrm{Cr}$, great sciatic: $P n$, vagus in the lower part of the neck; $r$, recurrent nerve winding round the subclavian artery.

Sympathetic Cord: $c$, Superior cervical ganglion; $c^{\prime}$, second or middle; $c^{\prime \prime}$, inferior; from each of these ganglia cardiac nerves (all deep on this side) are seen descending to the cardiac plexus; $d \mathrm{I}$, placed immediately below the first dorsal sympathetic ganglion; $d 6$ is opposite the sixth; $l \mathrm{I}$, first lumbar ganglion; $c g$, the terminal or coccygeal ganglion.

Preaortic and Visceral Plexuses: $p p$, pharyngeal, and, lower down, laryngeal plexus; $p l$, posterior pulmonary plexusspreading from the vagus on the back of the right bronchus; $c a$, on the aorta, the cardiac plexus, toward which, in addition to the cardiac nerve from the three cervical sympathetic ganglia, other branches are 
middle, and inferior cervical; twelve in the dorsal region; four in the lumbar region, and four in the sacral region.

Each ganglion is considered as a distinct center, receiving and giving branches in four different directions-viz., superior, or ascending, to communicate with the ganglion above; inferior, or descending, to communicate with the ganglion below; external, to communicate with the spinal nerves; and internal, to communicate with the sympathetic filaments of the opposite side and become distributed to the viscera.

Fig. I 4 illustrates the several portions of the sympathetic nerve. As has been said, this nerve is one of organic life, for there is no organ in the whole body but becomes affected when the function of this nerve is disturbed; from this it may be seen how sympathy exists between the several structures; this has already been dealt with in another part of this chapter.

seen descending from the vagus and recurrent nerves; $c 0$, right, or posterior, and $c o^{\prime}$, left, or anterior, coronary plexus; $o$, esophageal plexus in long meshes on the gullet; $s p$, great splanchnic nerve formed by branches from the fifth, sixth, seventh, eighth, and ninth dorsal ganglia; + , small splanchnic from the ninth and tenth; ++ , smallest, or third, splanchnic from the eleventh; the first and second of these are shown joining the solar plexus, so; the third descending to the renal plexus, $r e$; connecting branches between the solar plexus and the vagi are also represented- $-p n^{\prime}$, above the place where the right vagus passes to the lower or posterior surface of the stomach; $p n^{\prime \prime}$, the left distributed on the anterior or upper surface of the cardiac portion of the organ: from the solar plexus large branches are seen surrounding the arteries of the celiac axis, and descending to $m s$, the superior mesenteric plexus; opposite this is an indication of the suprarenal plexus; below re (the renal plexus) the spermatic plexus is also indicated; ao, on the front of the aorta, marks the aortic plexus, formed by nerves descending from the solar and superior mesenteric plexuses and from the lumbar ganglia; $m i$, the inferior mesenteric plexus surrounding the corresponding artery; $h y$, hypogastric plexus placed between the common iliac vessels, connected above with the aortic plexus, receiving nerves from the lower lumbar ganglia, and dividing below into the right and left pelvic or inferior hypogastric plexuses; $p l$, the right pelvic plexus; from this the nerves descending are joined by those from the plexus on the superior hemorrhoidal vessels, $m i^{\prime}$, by nerves from the sacral ganglia, and by visceral nerves from the third and fourth sacral spinal nerves, and there are thus formed the rectal, vesical, and other plexuses, which ramify upon the viscera, as toward $i r$, and $v$, the rectum and bladder. 


\section{REVIEW QUESTIONS}

What constitutes the nervous system?

What is the brain, and of how many parts does it consist?

What is the average weight of the brain?

What membranes surround the brain? Name them.

Describe the several membranes of the brain.

What part of the brain does the cerebrum form?

What important connection has the arachnoid membrane?

What is the relation of the pia mater?

Give the relations of the corpus callosum.

Where is the cerebrum located?

How does the medulla oblongata vary from the cerebrum as to sensitiveness?

Through what foramen does the spinal cord pass?

What is the average length of the spinal cord?

How is the spinal cord divided?

What is the circle of Willis?

What are sinuses, and what are their functions?

How many pairs of nerves are there?

How many pairs originate from the cranial region?

How many pairs originate from the spinal cord?

How many roots do the spinal nerves have?

What is considered to be the center of the nervous system?

What is the function of the cerebrum? Of the cerebellum?

What important relation has the medulla oblongata?

What are the functions of the spinal nerves?

Into how many groups are nerves divided?

What are the nerves of special sensation?

What are the nerves of general sensation?

What are the nerves of motion?

What nerves are involved in respiration?

What is the sympathetic nerve? What does it mantain?

What are ganglia?

How is the fifth cranial nerve divided?

What is the nerve of smell? Of sight? Of taste?

What is the function of the auditory nerve?

Give the function of the glossopharyngeal nerve.

What is the tenth nerve called?

What is the brachial plexus? How is it constructed?

What is the function of the intercostal nerves?

What do the lumbar nerves form?

What parts do the sacral nerves supply?

What is an efferent nerve? An afferent nerve? 


\section{CHA PTER VIII}

\section{THE GLANDULAR SYSTEM}

THE glands are secretory organs of diverse character and location. Some of them, as the liver and kidneys, are large, while others, such as the conglobate or lymphatic glands, are quite small. The mucous membranes that line the glands also perform the function of secretion, for they secrete the mucus in which they abound, as is seen in the mouth, nostrils, throughout the alimentary canal, and in the urinary organs. We will now consider these structures, and also some of the soft bulbous organs that perform the function of secretion.

\section{THE LYMPHATIC GLANDS}

The lymphatic glands are found distributed over all the body, and consist of numerous small globular bodies connected with the lymphatic vessels.

The lymphatic glands are most commonly called conglobate glands. They have no excretory duct except the continuation of the lymphatic vessels. The largest of the conglobate glands are the mesenteric, inguinal, and axillary glands. These glands are not believed to secrete material into the lymphatic system, but simply to alter the character of the fluid passing through them. In this sense they are considered the outposts of the body, defending it against injurious substances that are carried into the lymphatics. When poison or any irritating substance is forced into the system, as into the extremities, these 


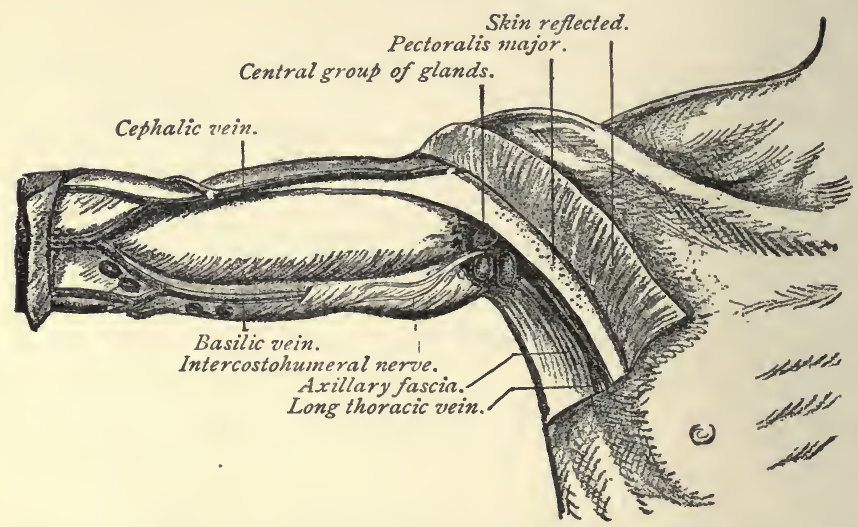

FIG. I15.-Central (superficial) lymphatic glands of the axilla (after Leaf).

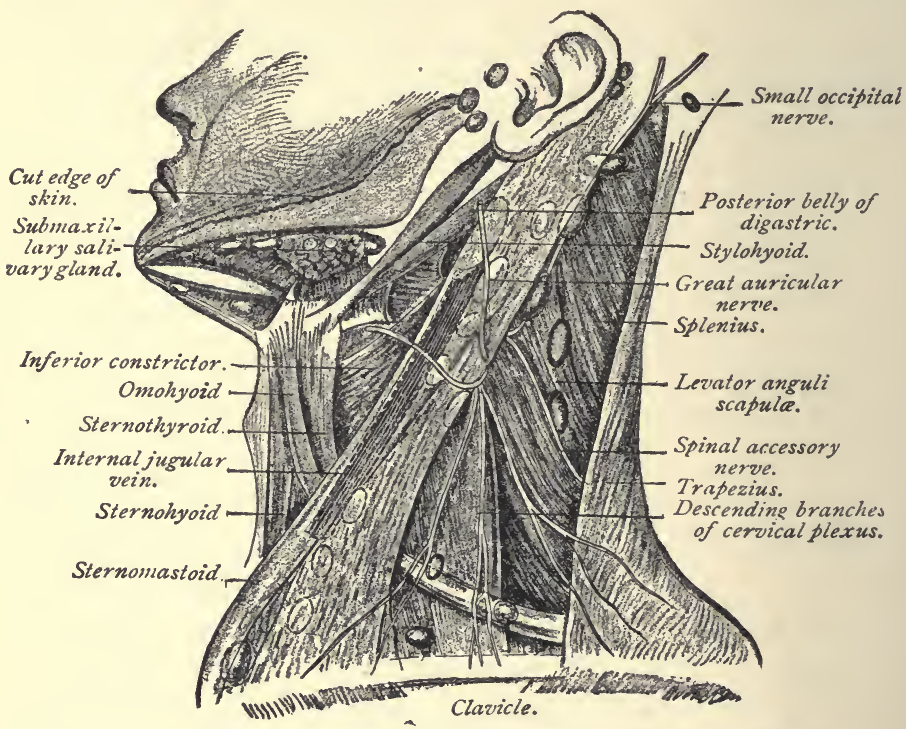

FIG. Ir6.-Lymphatic glands of the head and neck (after Leaf).

glands, situated between the entrance of the poison and the body, become swollen and inflamed, and absorb 
the foreign substance into their cells and retain and neutralize it; or, by softening and suppuration, they

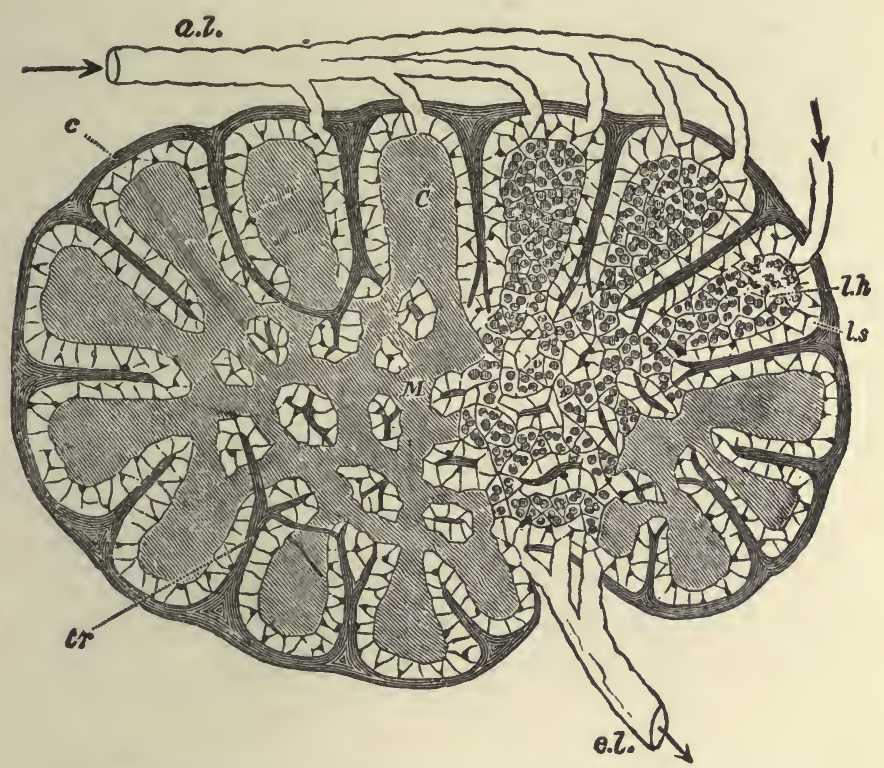

FIG. 117.-Diagram of a lymphatic gland, showing afferent (a.l.) and efferent $($ e. l.) lymphatic vessels; cortical substance $(C)$; medullary substance $(M)$; fibrous coat $(c)$; sending trabeculæ $(t r)$ into the substance of the gland, where they branch, and in the medullary part form a reticulum; the trabeculæ are surrounded by the lymph-path or sinus $(l . s)$, which separates them from the adenoid tissue $(l . h)$ (Sharpey).

"break out," and thus, by a discharge, expel the offensive material.

\section{THE LIVER}

The liver occupies part of the right hypochondriac and part of the epigastric region, immediately below the diaphragm. It is the largest gland in the body, and weighs about $4 \frac{1}{2}$ pounds. It is lobular in form, consisting 
mainly of two large and two smaller lobes. The gallbladder and its ducts are situated on the under surface of the liver. This organ will be subsequently described.

Function of the Liver.-Its chief function is to secrete bile, a necessary agent in the process of digestion. It also forms glycogen, assists in the formation of urea and allied products, and modifies the blood as it passes through it.

Bile is both a secretion and an excretion; it contains but few constituents other than those that are formed in the liver, and, as has been said, it is destined to play an im-

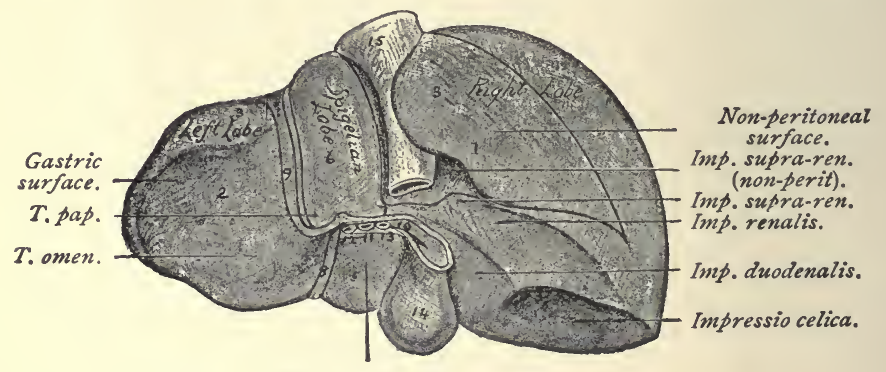

Impressio pylorica.

FIG. II8.-Posterior and inferior surfaces of the liver (Nancrede).

portant part in the process of nutrition. The other secretions of the liver are waste products that are associated with the bile.

The color of the liver is different from that of all the other glands. It is dark brown, and sometimes stained yellow with bile.

Blood=supply. - The liver is abundantly supplied with blood, and this blood is modified very materially in its passage through the gland.

The blood-vessels that enter the liver are the portal vein, made up of the gastric, splenic, superior, and inferior mesenteric veins. The hepatic artery supplies the re- 
quired amount of blood to the structure; the hepatic veins, originating in the interior, collect all the blood

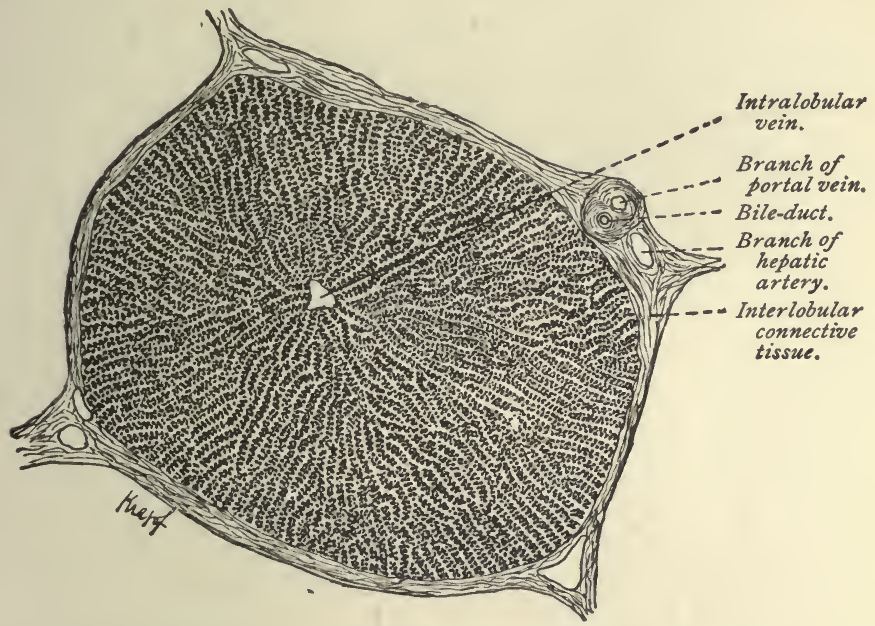

FIG. II9. - Section through liver of pig, showing chains of liver-cells $; \times 70$ (Böhm and Davidoff).

distributed by the portal vein and hepatic artery and conduct it to the ascending vena cava.

\section{THE GALL-BLADDER}

The gall-bladder is a pear-shaped sac, about 4 inches in length, situated in the sinus of the liver. It is a reservoir for the bile, and is capable of holding about $\mathrm{I} \frac{1}{2}$ ounces of fluid. It is made up of three coats-a serous, a fibrous, and a mucous.

\section{THE SPLEEN}

The spleen is an oblong gland situated in the left hypochondriac region, immediately below the diaphragm. 
It is of a spongy texture and of a reddish or violet color, about 5 inches in length, and weighs 6 ounces.

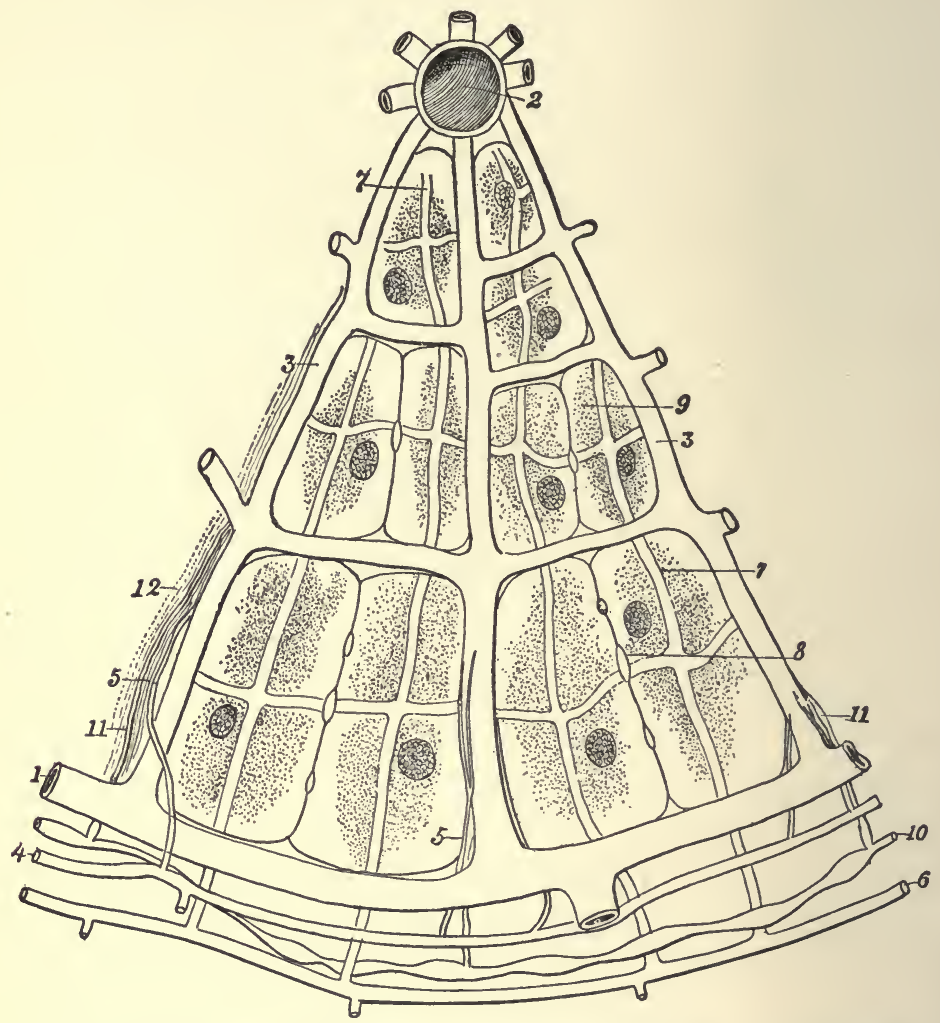

FIG. I20.-Diagram of a segment of a hepatic lobule: I, I, Interlobular portal vein; 2 , central vein ; 3,3 , intralobular capillaries; 4,4 , interlobular hepatic artery; 5, 5, ramifications of hepatic artery, contributing to the formation of the intralobular capillaries; 6,6 , interlobular bile-duct ; 7,7 , its ramifications in the lobule, forming a plexus of intercellular canaliculi; 8,8 , section of biliary canaliculi with their intercellular capillaries; 9,9 , hepatic cells; Io, Io, interlobular lymphatics, receiving the intralobular lymphatics; II; II, I2, intralobular connective tissue (Testut).

Function of the Spleen.-The spleen is probably concerned in the preparation of albuminous food for 
nutrition. During digestion the spleen becomes larger and its contents are increased in amount; after digestion it gradually diminishes in size, returning to its normal condition.

It is probably in this organ that the red corpuscles, after having fulfilled their function in the blood, are

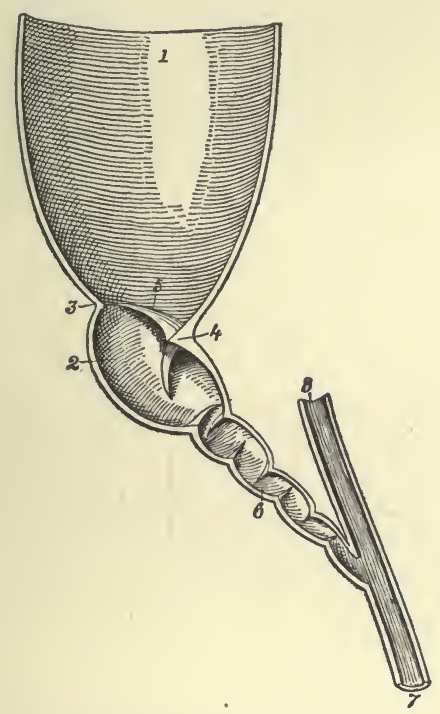

FIG. I21.-Portion of gall-bladder and bile-ducts: I, Cavity of gallbladder; 2, cavity of calyx; 3 , groove separating the calyx from the bladder $; 4$, promontory; 5 , superior valve of caly $x ; 6$, cystic canal; 7, common bile-duct; 8 , hepatic duct (Testut).

disintegrated, for the splenic venous blood contains relatively a small number of them. The white corpuscles, however, appear to be increased in number, for the blood of the splenic vein contains an unusually large proportion. The spleen serves also as a reservoir for the blood when the portal circulation becomes obstructed. 
Certain it is that this gland, like the conglobate glands, under some forms of disease (for instance, those that result from infections, particularly malaria) becomes

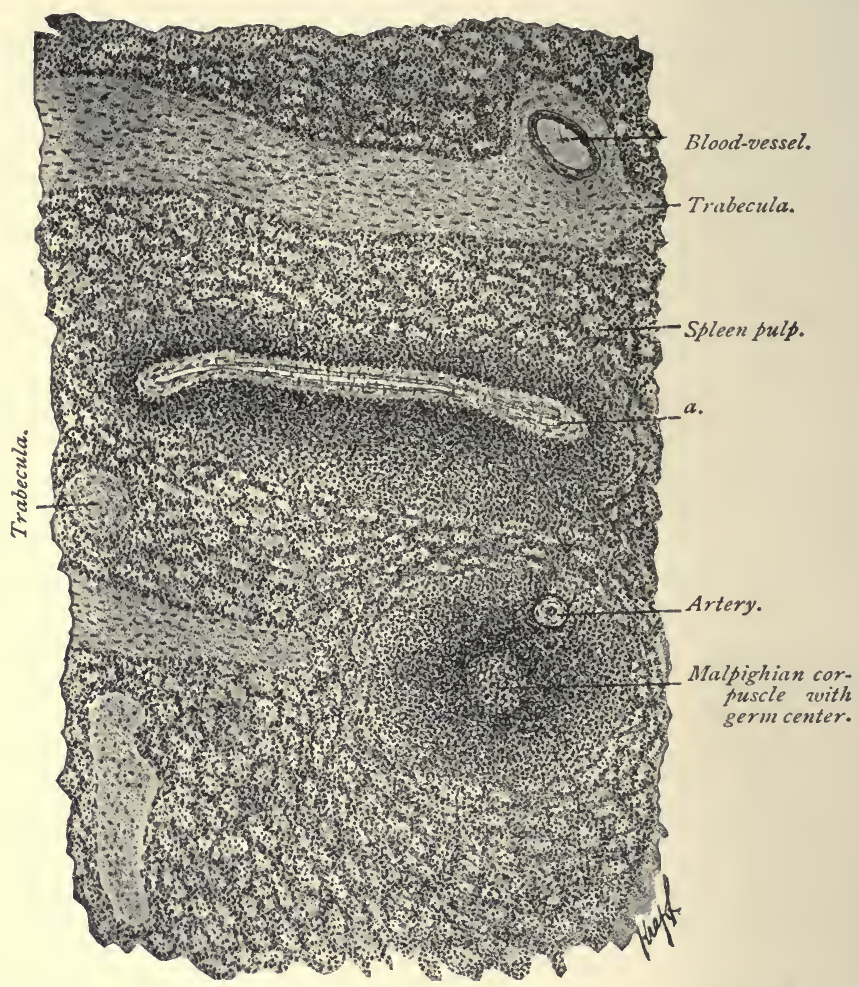

FIG. I22.-Part of a section through the human spleen : $\times 75$ (sublimate fixation). At $a$ is an oblong Malpighian body with a blood-vessel (Böhm and Davidoff).

inflamed and often indurated and swollen, thus exhibiting its office in modifying the character of some of the materials of the blood. 


\section{THE PANCREAS}

The pancreas is an oblong, soft, glandular body, situated transversely across the posterior wall of the abdomen, in the left hypochondriac region, just behind the stomach. It is about 6 inches long, and weighs about 4 ounces. Its office is to secrete the pancreatic juice,

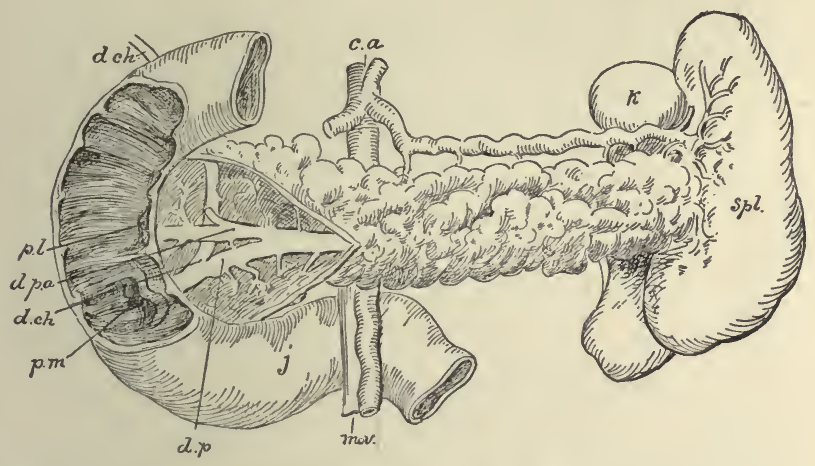

FIG. I23.-Pancreas dissected to show (d.p.) pancreatic duct; $d . p . a$, accessory duct; $d$. ch, bile-duct. Duodenum laid open to show $(p . m$. papilla major; $p . l$, papilla minor; $s p l$. , spleen; $k$, kidney; $j$, jejunum; $m$. $v$, mesenteric vessels; $c$. $a$, celiac axis (Robson and Moynihan).

which performs a part in the digestive process. The function of the pancreatic juice is to convert starch into maltose and albuminoids into peptones; it also effects the emulsification of fats.

\section{THE PAROTID GLANDS}

These are situated in front of the lower portion of the ear, just above the angle of the jaw, one on each side. They are small, soft bodies, and their office is to secrete the saliva, which affords the necessary moisture to the mouth and the requisite fluid for the process of mastication. The saliva is also an important agent in the func- 
tion of digestion. The practice of diluting the food with large quantities of water while eating is injurious, for drinking at such times prevents the flow of saliva into the mouth that would otherwise take place.

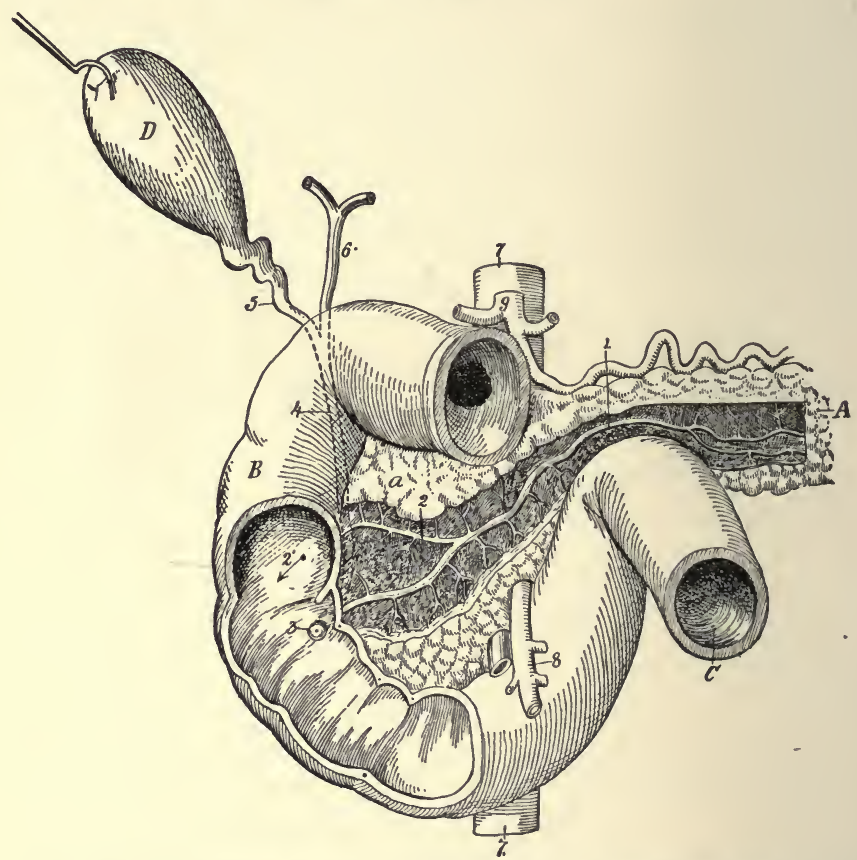

FIG. 124.-Excretory ducts of the pancreas: $A$, Pancreas, with $a$, its head; $B$, duodenum; $C$, jejunum; $D$, gall-bladder. $I$, main pancreatic duct of Wirsung ; 2 , accessory duct with $2^{\prime}$, its opening upon the posterointernal wall of the duodenum; 3 , ampulla of Vater; 4 , common bile-duct; 5 , cystic duct; 6 , hepatic duct; 7 , aorta; 8 , superior mesenteric vessels; 9 , celiac axis with its three branches (Testut).

The salivary duct opens into the mouth opposite the second molar tooth in the upper jaw. This is the gland that is affected in the disease known as mumps (epidemic parotitis). 


\section{THE SUBMAXILLARY GLANDS}

These glands are situated on the inner side of the lower jaw-one on each side of the mouth anterior to the angle of the jaw. Their excretory ducts open into the mouth

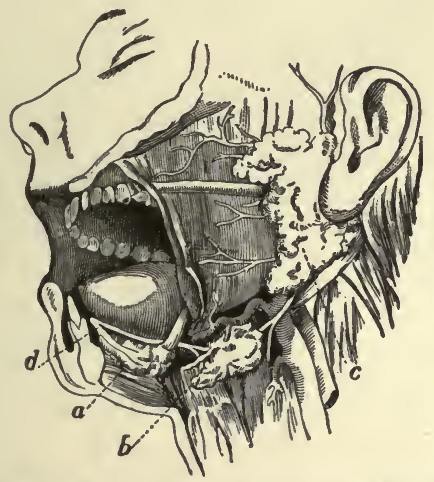

FIf. 125. Dissection of the side of the face, showing the salivary glands: $a$, Sublingual gland; $b$, submaxillary gland, with its duct opening on the floor of the mouth beneath the tongue at $d ; c$, parotid gland and its duct, which opens on the inner side of the cheek (after Yeo).

on each side of the frenum, or "thread" (bridle), of the tongue. The fluid secreted by them may sometimes be seen to ooze forth when the mouth is open, especially when the mind is allowed to dwell on the eating of fruits.

\section{THE SUBLINGUAL GLANDS}

The sublingual glands are situated beneath the tongue, within the lower jaw, one on each side of the "thread" of the tongue. They are small, elongated bodies, and lie immediately under the mucous membrane of the floor of the mouth. They have a number of ducts or openings that freely discharge the fluid secretion of the glands. Their function is similar to that of the parotid glands. 


\section{THE THYROID GLAND}

This is a small, flat, glandular body lying against the anterior surface of the trachea, below the thyroid cartilage. It is susceptible of great enlargement, and is often found abnormally enlarged, especially in women. This enlargement constitutes the disease called goiter, bronchocele, and sometimes "big neck."

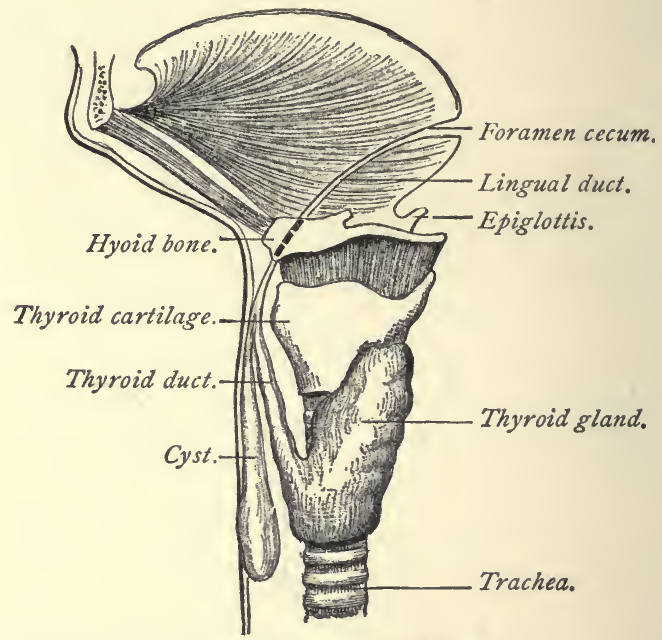

FIG. 125.-The thyroglossal duct (Marshall).

The thyroid gland, although supplied with four large arteries and veins and also with four nerves, has no excretory duct that has yet been discovered. Its function has never been thoroughly understood.

\section{THE LACRIMAL GLANDS}

These two small glands, which are situated, one upon each side, in a depression in the frontal bone at the upper and outer angle of the orbit. (See Fig. 127.) 
The size of the lacrimal glands is about that of the kernel of a peach-stone. They have many excretory

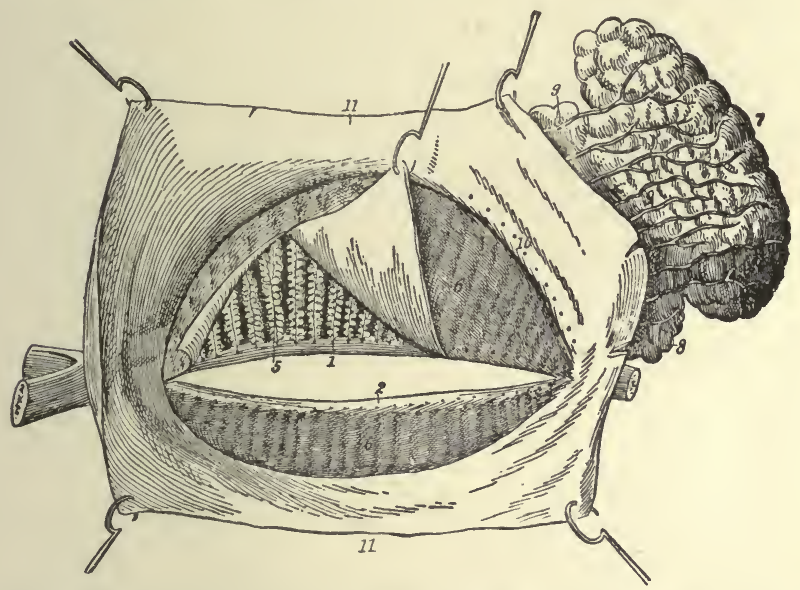

FIG. I27.-Lacrimal and Meibomian glands, the latter viewed from the posterior surface of the eyelids (the conjunction of the upper lid has been partially dissected off, and is raised so as to show the Meibomian glands beneath): $x$, Free border of upper, and 2 , free border of lower lid, with openings of the Meibomian glands; 5 , Meibomian glands exposed, and 6, as seen through conjunctiva; 7,8 , lacrimal gland; 9 , its excretory ducts, with $r o$, their openings in the conjunctival cul-de-sac; $I T$, conjunctiva.

ducts, which open into the eye under the upper lid. Their function is to furnish moisture to the eyes; these are the organs which secrete the tears.

\section{THE MESENTERIC GLANDS}

These glands are small, knot-like bodies, occurring in the course of the chyliferous vessels in the mesentery. They are very numerous, but have no excretory duct. The lacteals, however, as stated in a previous chapter, pass through them. It is not known certainly to what extent the chyle, conveyed through the glands by the lacteals, is modified; and hence the office of the mesenteric glands is as yet but little understood. 


\section{THE MAMMARY GLANDS}

The mammary glands, which secrete the milk, are two more or less hemispheric organs, situated in the human female on the anterior surface of the chest. Though rudimentary in childhood, they gradually increase in size as the female approaches puberty.

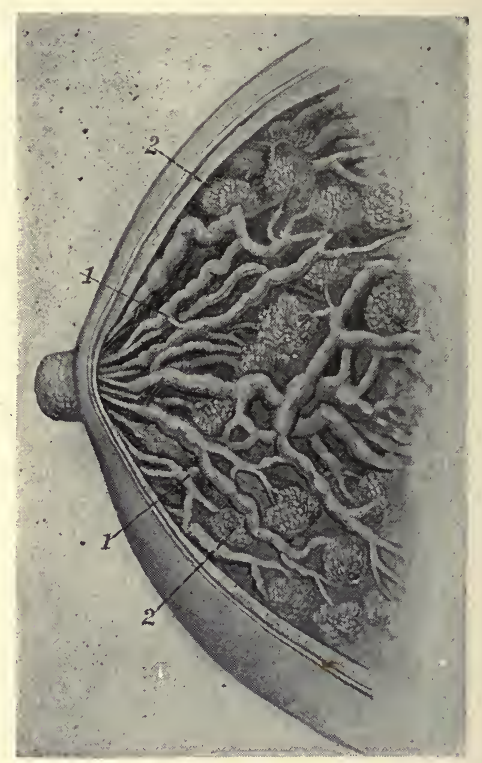

FIG. I28.-Mammary glands: I, Lacteal ducts ; 2, glandular acinus (after Playfair).

The gland presents at its convexity a small prominence of skin, the nipple, which is surrounded by a circular area of pigmented skin, the areola. The gland proper is covered anteriorly by a layer of adipose tissue, and attached posteriorly to the pectoral muscles by a meshwork of fibrous tissue. 
Changes in the Mammary Glands.-During pregnancy the mammary glands become larger, firmer, and more lobulated; the areola darkens, and the veins become more prominent. During the period of lactation the gland is the seat of active histologic and physiologic changes correlated with the production of milk. At the close of lactation the glands diminish in size, undergo involution, and gradually return to their original, non-secreting condition.

Structure of the Mammary Glands.-Each gland consists of an aggregation of some fifteen to twenty lobes, each of which is surrounded by a framework of fibrous tissue. The lobe is provided with an excretory duct, which, as it approaches the base of the nipple, expands to form a sinus or reservoir, beyond which it opens by a narrowed orifice on the surface of the nipple. On tracing the duct into the lobe it is found to divide and subdivide, and finally to terminate in lobules or acini. Each acinus consists of a basement membrane, lined by cells, and supplied with blood-vessels, lymphatics, and nerves.

\section{THE SEBACEOUS GLANDS}

The sebaceous glands are embedded in the true skin, and consist of grape-like masses opening by a duct upon the surface of the epidermis or into a hair-follicle. They are found in almost every part of the body, but most abundantly upon the face. They secrete an oily substance, known as sebum. It is obstruction of these ducts that produces what are commonly known as "blackheads," which are seen so often upon the face.

The deposit, so abundant upon some children at birth, and which consists of a white, glue-like substance, is the residue of the sebaceous matter, and is known as the vernix caseosa. 
248 ANATOMY AND PHYSIOLOGY FOR NURSES.
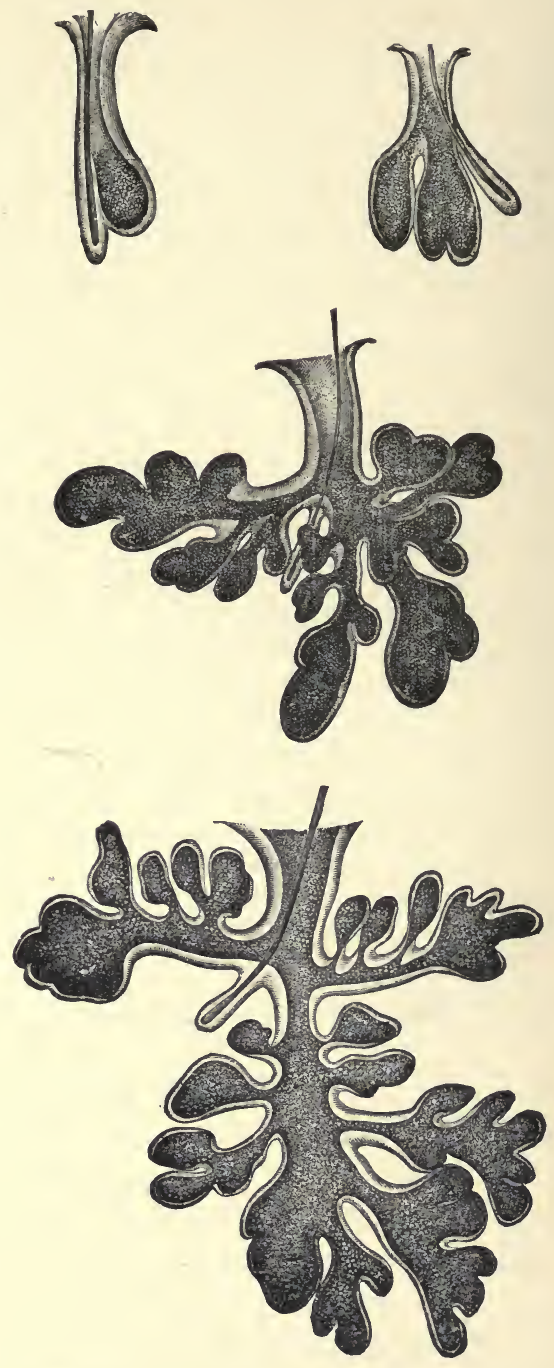

FIG. 129.-Sebaceous glands of the face-simple pouch to compound lobular, with lanugo hair and small or rudimentary hair-follicle, the largest from the nose (Sappey). 


\section{THE SUDORIPAROUS GLANDS}

The sudoriparous glands excrete the sweat. They consist of minute openings, commonly called the pores of the skin. Their function is to extract from the blood the excrementitious material (this function has been described in a previous chapter). It is estimated that the daily excretion from these glands amounts to about 2 pounds, although it varies according to the nature of the food and drink taken, the amount of exercise, external temperature, season, etc.
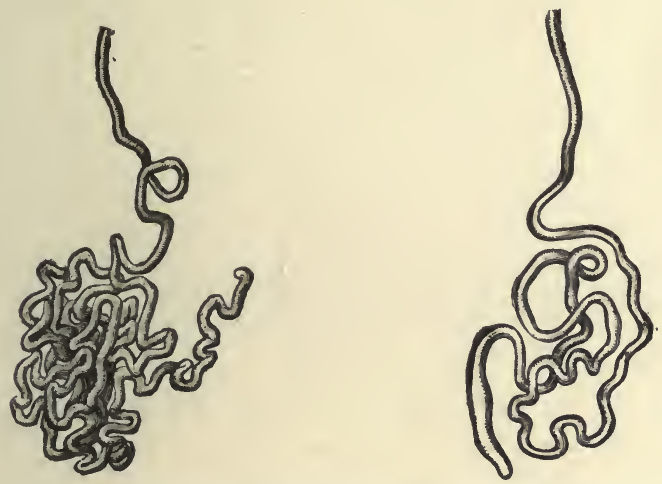

FIG. I30.-Sweat-glands of different size (of moderate magnification), showing coil or convolutions forming gland proper, the blind end of tubule, and excretory duct (Sappey).

The secretion of sweat is regulated by the nervous system. Here, as in the secreting glands, the fluid is formed from material in the lymph-spaces surrounding the glands. Two sets of nerves are concerned: Vasomotor, regulating the blood-supply; and secretory, stimulating the activities of the gland-cells. Generally, the two conditions, increased blood-flow and increased glandular action, coexist. At times a profuse, clammy perspiration occurs with a diminished blood-flow. 
Besides the glands already described, many others of minor importance occur. For a description of these, the reader is referred to the more extensive works on anatomy.

\section{REVIEW QUESTIONS}

What are glands?

What are lymphatic glands? What is their function?

Which is the largest gland of the body? Where is it located?

How many lobes are there in the liver?

What is the function of the liver?

What large vein enters the liver?

What artery supplies the liver?

What is the average weight of the liver?

What is the gall-bladder?

What is the function of the bile?

What is the capacity of the gall-bladder?

Where is the spleen situated, and what is its function?

What is the pancreas? Its function?

What is the function of the pancreatic juice?

Where are the parotid glands situated, and what is their function?

What disease is liable to attack them?

What duct carries the secretion from this gland, and where does it open?

Give location of the submaxillary glands. What is their function?

Give location of sublingual glands.

What is the thyroid gland? State location.

To what disease is this gland most susceptible?

What is the prostate gland?

Give the function of the lacrimal glands and state their location.

Where do we find the mesenteric glands? What is their function?

What are the mammary glands? Where situated?

What are the functions of these glands?

What changes take place in these glands?

Describe the structure of the mammary glands.

What are the sebaceous glands?

Describe the location of the sudoriparous glands.

How is the secretion of the various glands regulated? 


\section{CHAPTER IX}

\section{THE MEMBRANES OF THE BODY}

THE mucous membrane is the proper lining of the alimentary canal, the respiratory, the urinary, and the genital organs. It is of a glandular construction, and in some parts secretes mucus copiously. This membrane, like the skin, has numerous openings upon its surface,

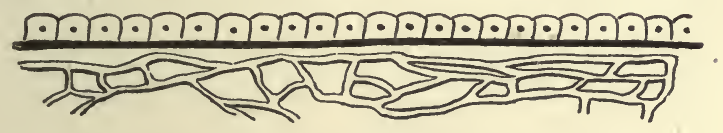

FIG. I3I.-Plan of a secreting membrane (Howell).

through which a large amount of fluid materials are removed, as is seen in diarrhea and dysentery. The urinary bladder, which is of a fibrous texture in its main construction, has a thick mucous membrane that protects the organ against irritation from the acrid substances in the urine.

The serous membranes are found on internal surfaces that have no outlets, as the pleura, peritoneum, pericardium, and the brain. These membranes serve to support, in their various places and positions, the several organs they invest.

The pericardium forms a sac enclosing the heart; the peritoneum invests the contents of the abdominal cavity; and the pleura forms two sacs to support the lungs. They all secrete a fluid for their lubrication.

From the serous membranes a serous or watery fluid is exuded, which affords a moist and smooth surface for 
the play of the several organs upon them. These membranes are of a light-red color and well supplied with blood-vessels. In some places they are liable to serious inflammation, often forming adhesions to other organs.

Synovial membranes resemble the serous membranes in structure, but differ from them in the nature of their secretion, which in the former is thick, viscid, and glairy, like the white of an egg, hence called synovia.

Synovial membrane is a thin, delicate membrane, arranged in the form of a short, wide tube, attached by its open ends to the margins of the articular extremities of the bones, and covering the inner surface of the various ligaments that connect the articulating surfaces.

The synovial membranes found in the body admit of subdivision into three kinds-articular, bursal, and vaginal. The articular synovial membranes are found in all movable joints; the bursce are found interposed between surfaces that move upon each other, producing friction, as the gliding of a tendon or of the integument over projecting surfaces; the vaginal serve to facilitate the gliding of tendons in the osseofibrous canals, through which they pass, as in the hand and foot.

The membranes of the brain have been described in the chapter on the Nervous System.

\section{REVIEW QUESTIONS}

What kind of membranes have we in the body?

What cavities are lined by mucous membranes?

Name the serous membranes.

What are synovial membranes? Note the varieties.

What membrane lines the heart?

What membrane surrounds the lungs?

What is the function of the serous membranes? 


\section{THE ORGANS OF SPECIAL SENSE}

\section{THE ORGANS OF SIGHT}

THE eyes are two globular bodies, situated one in each orbit. They are the organs of vision, and are enclosed partly by the lids and partly by the bony sockets.

The eyeball is composed of several tunics or coats. In front we have the conjunctiva, then the sclerotic coat, or

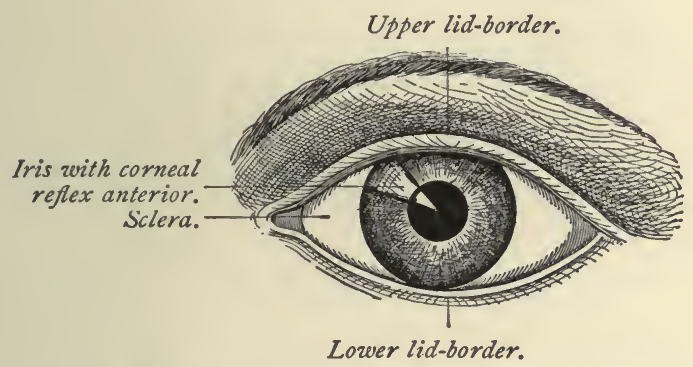

FIG. 132.-The anterior surface of the eyeball (Pyle).

white firm structure that surrounds all the ball except the front, where the cornea takes its place. The cornea is a transparent structure that admits the rays of light for vision. Then follow the choroid coat, iris, and ciliary processes, together constituting the second or middle coat of the eyeball. The humors or fluids are the aqueous, crystalline, and vitreous.

The sclerotic coat is a dense, fibrous layer that invests about the posterior five-sixths of the globe of the eye. 
It gives form to this organ, and serves for the attachment of the muscles that move the eye in various directions. This coat, from the brilliancy of its whiteness, is known as "the white of the eye." Anteriorly, the sclerotic coat presents a beveled edge, which receives the cornea in the same way that a watch-glass is received by the groove in the case.

The cornea is the transparent projecting layer that forms the anterior sixth of the globe of the eye. In

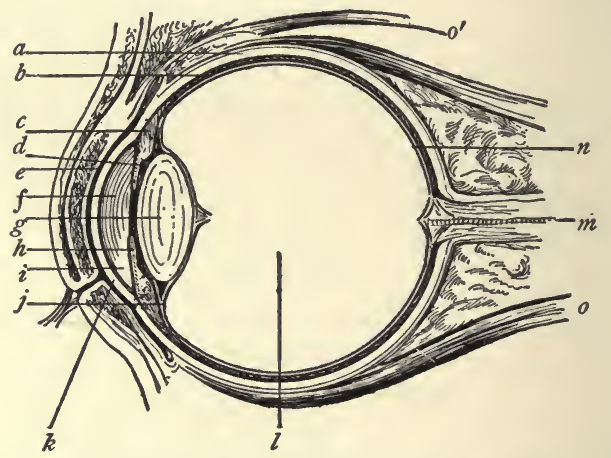

FIG. I33--Vertical section through the eyeball : $a$, Sclera; $b$, choroid; $c$, ciliary muscle; $d$, iris; $e$, cul-de-sac of conjunctiva; $f$, anterior chamber and aqueous humor; $g$, crystalline lens; $h$, posterior chamber $; i$, angle of anterior chamber; $j$, suspensory ligament of lens; $k$, cornea; $l$, vitreous; $m$, optic nerve with central artery of retina; $n$, retina; $0, o^{\prime}$, ocular muscles (Pyle).

form it is circular, convexoconcave, and resembles a watch-glass. It is received by its edge, which is sharp and thin, within the beveled border of the sclerotic, to which it is firmly attached. The cornea is composed of several different layers; its blood-vessels are so small that they exclude the red particles altogether and admit nothing but serum.

The choroid coat is a vascular structure, of a rich, chocolate-brown color upon its external surface, and of a 
deep-black color within. It is connected externally with the sclerotic, by an extremely fine cellular tissue and by the passage of the nerves and vessels; internally, it is in contact with the retina. The choroid membrane is composed of three layers. It secretes upon its internal surface a dark substance, called pigmentum nigrum, which is of great importance in the function of vision.

The iris, so called from the variety of its colors in different persons, forms a partition between the anterior

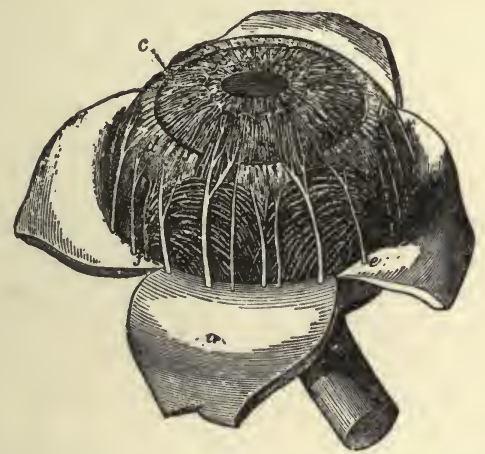

FIG. I34-Choroid membrane and iris, exposed by the removal of the sclerotic and cornea: $a$, One of the segments of the sclerotic thrown back; $b$, ciliary muscle; $c$, iris; $e$, one of the ciliary nerves; $f$, one of the vasa vorticosa or choroidal veins (Zinn).

and posterior chambers of the eye, and is pierced by a circular opening called the pupil. It is composed of two layers. The radiating fibers of the anterior layer converge from the circumference to the center. Through the action of these radiating fibers the pupil is dilated. The circular fibers surround the pupil, and their action produces contraction of the area. The posterior layer is of deep-purple tint, and is called uvea, from its resemblance in color to a ripe grape.

The ciliary processes consist of a number of tri- 
angular folds, formed, apparently, by the plaiting of the internal layer of the choroid coat; they are about sixty in number. Their external border is continuous with the internal layer of the choroid coat. The central border is free, and rests against the circumference of the crystalline lens. These processes are covered by a layer of pigmentum nigrum.

The retina is composed of three layers: the external, the middle or nervous, and the internal or vascular.

The external layer is extremely thin, and the membrane is seen as a flocculent film when the eye is suspended in water.

The nervous membrane is the expansion of the optic nerve, and forms a thin, semitransparent, bluish-white layer. The vascular membrane consists of the ramifications of a minute artery and its accompanying vein. The vascular layer forms distinct sheaths for the nervous papillæ, which constitute the inner surface of the retina.

The aqueous humor fills the anterior and posterior chambers of the eye. It is an albuminous fluid, having an alkaline reaction. Its specific gravity is a very little greater than that of distilled water. The anterior chamber is the space intervening between the cornea in front, and the iris and the pupil behind; the posterior chamber is the narrow space, less than half a line in depth, bounded by the posterior surface of the iris and pupil in front, and by the ciliary processes and crystalline lens behind. The two chambers are lined by a thin layer-the secreting membrane of the aqueous humor.

The crystalline humor, or lens, is situated immediately behind the pupil, and is surrounded by the ciliary processes. This humor is more convex on the posterior than on the anterior surface, and in different portions of the surface of each the convexity varies. The lens is embedded in the anterior part of the vitreous humor, from 
which it is separated by a thin membrane; it is invested by a transparent elastic membrane, called the capsule of the lens. The lens consists of concentric layers arranged like the coats of an onion. The external layer is soft, and each successive one increases in firmness until the central layer forms a hardened nucleus. These layers are best demonstrated by boiling or by immersing in alcohol, when they easily separate from one another.

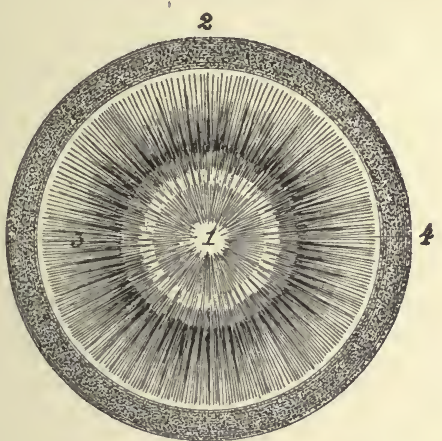

FIG. I35-Crystalline lens and suspensory ligament or zonula: I, Lens ; 2 , posterior, and 3 , anterior portion of zonula; 4 , its insertion into the preequatorial region. The black rays are lines of pigment torn from the ciliary processes, and belong in reality to the ciliary portion of the retina (Testut).

The vitreous humor forms the principal bulk of the globe of the eye. It is an albuminous fluid, resembling the aqueous humor, but is denser, and differs from the aqueous in this important particular, that it has not the power of reproducing itself. If, therefore, by accident it is discharged, the eye is irrecoverably lost; whereas, if the aqueous humor is discharged, it will again be restored. The vitreous is enclosed in a delicate membrane, called the hyaloid, which sends processes into the interior of the globe of the eye, forming the cells in which the humor is retained. 


\section{THE APPENDAGES OF THE EYE}

The appendages of the eye are the eyebrows, eyelids, eyelashes, conjunctiva, caruncula lacrimalis, and lacrimal apparatus.

The eyebrows (supercilia) are two prominent arches of integument, covered with short, thick hairs, which form the upper boundary of the orbits. They serve to shade the eyes from a too vivid light, and protect them from the particles of dust and moisture that roll down the forehead.

The eyelids (palpebræ) are two valvular layers placed in front of the eye, serving, by their closure, to defend it against injury; they have been called the "blinds of the eye." When drawn up, they disclose an elliptic space (fissura palpebrarum), the angle of which forms the outer and inner canthi. The inner canthus is prolonged for a short distance inward toward the nose, and a triangular space is formed which is called the lacus lachrymalis. At the commencement of the lacus lachrymalis, upon each of the two lids, is a small, angular projection, the papilla or tubercle, which forms the entrance to the lacrimal canal.

The tegumentary areolar tissue of the eyelids is remarkable for its looseness and absence of fat; it is particularly liable to serous infiltration after injury to these parts.

The tarsal cartilages contribute to the support of the eyelids. They are semilunar in form, the superior being about $\frac{1}{3}$ inch in breadth at its middle and tapering toward each extremity. The inferior is an elliptic band, narrower than the superior, and is situated in the substance of the lower lid. Its upper border is flat, and corresponds with the edge of the upper cartilage. The lower is held in place by the fibrous membrane, which is firmly attached to the periosteum around the margin of the orbit. 
The Meibomian glands are embedded in the substance of the cartilages, and are distinctly seen on examining the inner aspect of the lids. They have the appearance of a string of pearls, and are about thirty in number in the upper cartilage, and somewhat fewer in the lower; they open by minute foramina on the edges of the lids. (See p. 245.)

The edges of the eyelids are provided with a row of long, thick hairs (eyelashes) that curve upward from the

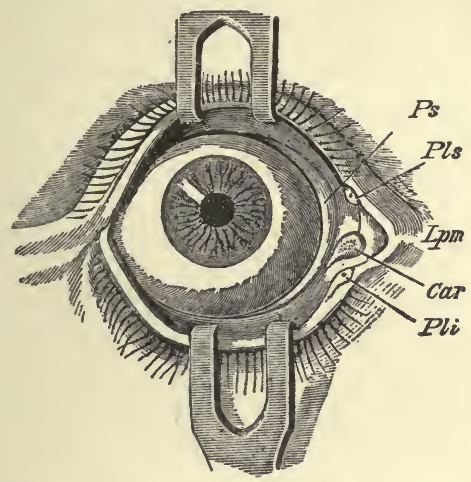

FIG. 136.-Right eye from before, the eyelids separated by hooks: $P$ s, Plica semilunaris; $P l s, P l i$, superior and inferior lacrimal puncta; Car, lacrimal caruncle; $L p m$, internal tarsal ligament.

upper lid and downward from the lower, so as not to interlace with one another when the eyelids close.

The conjunctiva is the mucous membrane of the eye. It covers the whole anterior surface, with the exception of the cornea, which it, however, provides with an epithelial layer, and is reflected backward, upward, and downward upon the lids so as to form their internal layer.

The caruncula lacrimalis is a small, reddish body that occupies the inner canthus of the eye. In health it presents a bright-pink tint; in sickness it loses its color and becomes pale. 
The nasal duct, a part of the lacrimal apparatus, is a short canal, about $\frac{3}{4}$ inch in length, directed downward, backward, and a little outward to the inferior meatus of the nose, where it terminates by an expanded orifice. It is provided with a mucous membrane, continuous with the conjunctiva above and with the membrane of the nose below. Obstruction of this duct from inflammation and suppuration leads to the formation of a lacrimal fisiula. The nerves and vessels are derived from the ophthalmic branches.

\section{PHYSIOLOGY OF VISION}

After reviewing the anatomic relation of sight, we will now take up a few essential points regarding the physiologic action of the various structures.

It is by the sense of sight that we receive the impression of light and color, as well as the ideas of construction of materials, establishing certain fancies, likes, and dislikes of the external surroundings.

The eyeball is constructed like a photographer's camera. The sclerotic and choroid coats correspond to the inside walls of the chamber, while by the refractive power of the several parts, as the cornea, aqueous humor, crystalline lens, and vitreous humor, the image is formed. The retina represents the sensitive plate on which the image is received. The iris regulates the rays of light, acting as a diaphragm to cut out certain portions of light that are not required. The ciliary muscles adjust the shape of the crystalline lens so as to focus images upon the retina.

The function of the crystalline lens is to focus the rays of light with the formation on the retina of the image, corresponding with the object from which the light proceeds. By accommodation is meant the power of adjusting the eye to certain distances. A normal eye does not 
require adjustment for parallel rays, but for divergent rays a change in the eye is necessary; the ability to make this change is known as the power of accommodation.

The astigmatic eye is so called from the inability. to focus vertical and horizontal lines at the same time. 'This is usually due to some irregularity of the curvature of the refracting surfaces of the eye, and a cylindric lens is required to correct the defect.

Myopia, or short-sightedness, is a condition caused an increased anteroposterior diameter of the eyebdll, causing the parallel rays of light to focus in front of the retina. Myopia causes diminution of distance vision, and requires concave lenses to correct the deficiency.

Hyperopia, or far-sightedness, is a condition of refraction of the eye in which, when the accommodation is at rest, the focus of the parallel rays of light transmitted through the eye is beyoild the retina. Convex glasses are required to correct this defect.

Presbyopia is a condition of the eye in which the power of accommodation is partly or wholly lost. This condition occurs in the aged, usually commencing between the ages of forty and fifty years, and requires convex spheric lenses for its correction.

\section{THE ORGAN OF HEARING}

The ear is the organ of hearing, and consists of a series of cavities so arranged as to receive the vibrations (sounds) in the atmosphere, and convey them to the delicate nervous membrane within its structure. The ear is composed of three parts: the external ear, the tympanum or middle ear, the labyrinth or internal ear.

The external ear is composed of two parts - the pinna (auricle or pavilion of the ear) and the external auditory meatus (auditory canal).

The pinna is a cartilaginous plate that surrounds the 
entrance of the auditory canal. It presents several ridges and furrows, arising from the folds of the cartilages that form it.

The external auditory meatus is a canal, partly cartilaginous and partly bony, about I inch in length, whose walls extend inward from the pinna to the membrana tympani, or drum of the ear. It is narrower in the middle than at the extremities. It is lined by an extremely thin pouch of cuticle, which, when withdrawn after maceration, pre-

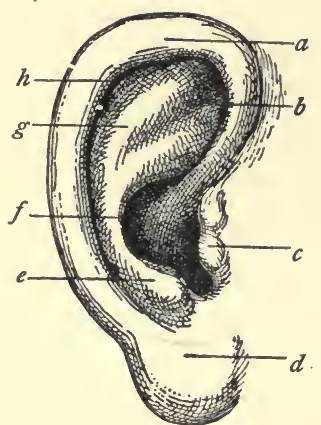

FIG. I37.-The external ear: $a$, Helix; $b$, fossa of anthelix; $c$, tragus; $d$, lobule; $e$, antitragus; $f$, concha; $g$, anthelix; $h$, fossa of helix (Randall). serves the form of the canal. Stiff, short hairs that stretch across the tube are often found in the interior of the channel, and prevent the ingress of insects. Beneath the cuticle are a number of small follicles that secrete the wax (cerumen) of the ear.

The membrana tympani is a thin, semitransparent membrane of an oval shape. It is about $\frac{3}{8}$ inch in dimeter, and is inserted into the groove around the circumference of the meatus, near its termination. This membrane is placed obliquely across the tube. It is concave toward the meatus and convex toward the tympanum.

The tympanum is an irregular bony cavity, situated within the temporal bone. It is bounded externally by the membrana tympani; internally, by the inner wall; and in its circumference, by the petrous portion of the temporal bone and mastoid cells. The tympanum is traversed by a chain made of three small bones-the malleus, incus, and stapes. (See Fig. I39.)

The mastoid cells are very numerous, and occupy the 
whole of the interior of the mastoid process and part of the petrous portion of the temporal bone. They com-

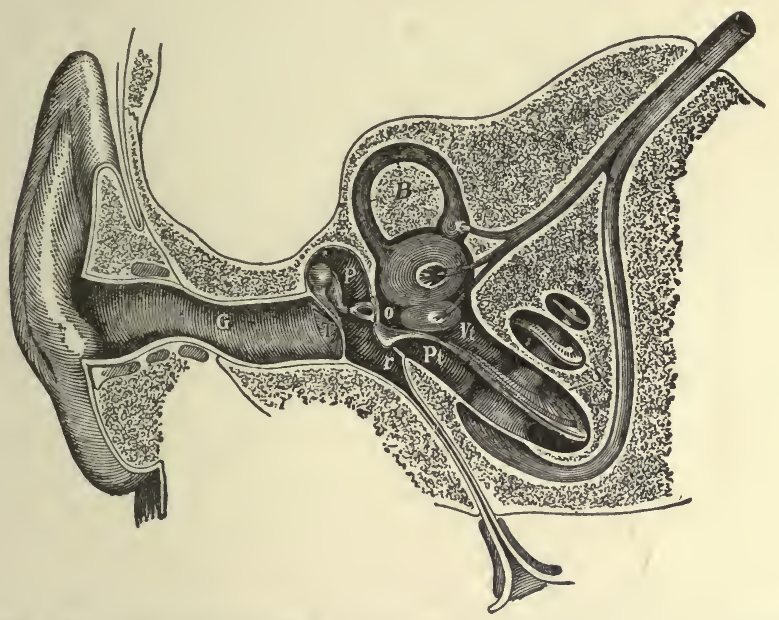

FIG. 138.-Semidiagrammatic section through the right ear: $G$, external auditory meatus; $T$, membrana tympani ; $P$, tympanic cavity ; $o$, fenestra ovalis; $r$, fenestra rotunda; $B$, semicircular canal; $S$, cochlea; $V t$, scala vestibuli; $P t$, scala tympani (Czermak).

municate with the upper and posterior circumference of the tympanum by a large irregular opening.

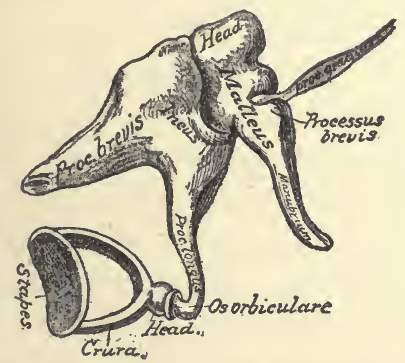

FIG. 139.-The small bones of the ear; external view (enlarged).(after Gray).

The Eustachian tube is a canal of communication, extending obliquely between the pharynx and the anterior 
circumference of the tympanum. In structure it is partly fibrocartilaginous and partly bony. It is broad and expanded at its pharyngeal extremity, and narrow and compressed at the tympanum.

The nerve supply is mainly from the auditory nerve.

The internal ear, or labyrinth, is the essential part of the organ of hearing. It consists of three partsthe vestibule, semicircular canals, and cochlea. It receives the ultimate distribution of the auditory nerve. It is connected with the middle ear by two openings, the fenestra rotunda and the fenestra ovalis.

(The nurse will do well to devote some time to the study of these organs, for she will often be called upon to care for patients in whom these important parts are diseased.)

\section{THE ORGANS OF TASTE}

The chief organ of taste is the tongue, and yet the palate and lips participate in receiving the impressions made by substances taken into the mouth. These impressions are conveyed to the brain by the nerves, and thus a perception of the quality of the taste or impression on the tongue is effected.

The tongue is a double organ, composed chiefly of muscular fibers, which run in almost every direction. The two sides are absolutely distinct, so that sometimes, as in paralysis, one side is affected while the function of the other remains unimpaired. It possesses great versatility of motion, and can be molded into a variety of shapes. The tongue is an auxiliary to other organs in articulation, mastication, and deglutition.

This organ is abundantly supplied with blood-vessels, a large artery being sent to each side of it. It is also well furnished with nerves, receiving nervous filaments from the fifth, ninth, and twelfth pairs of nerves. The branch of the fifth, formerly called the gustatory, from its 
fancied connection with the sense of taste, is the nerve of common sensation for the tongue. The ninth or glosso-

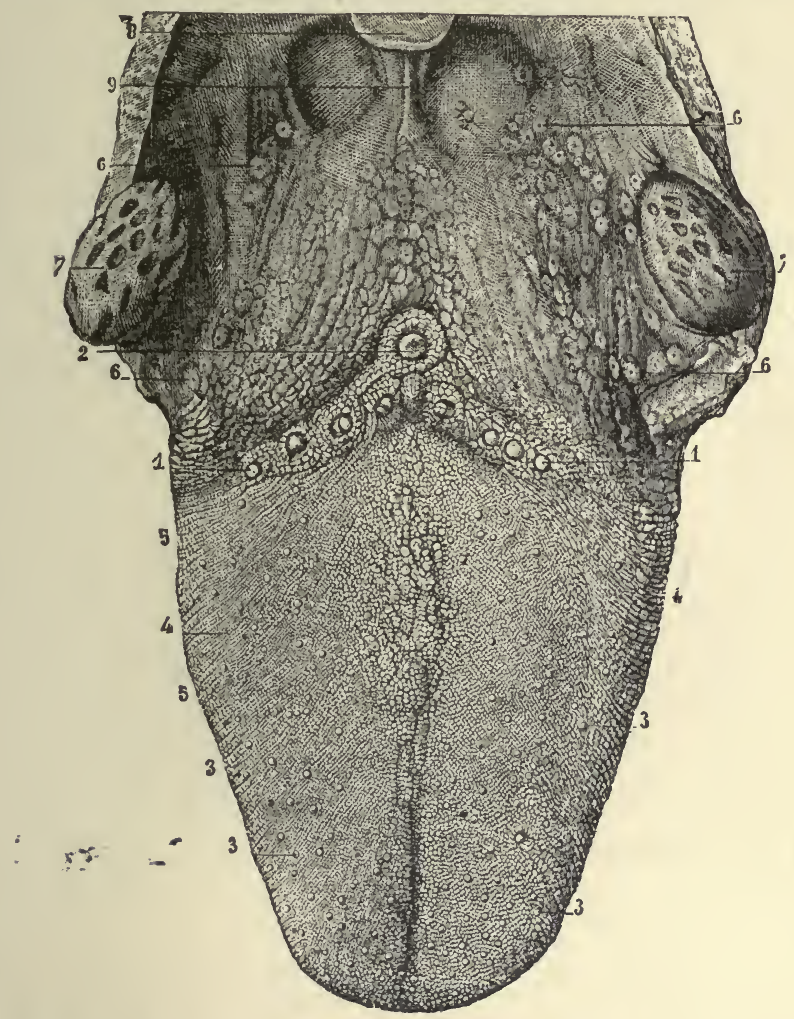

FIG. I40.-The upper surface of the tongue, with the fauces and tonsils : I, I, Circumvallate papillæ, in front of 2 , the foramen cæcum ; 3 , fungiform papillæ; 4, filiform and corneal papillæ; 5 , transverse and oblique rugæ; 6 , mucous glands at the base of the tongue and in the fauces; 7 , tonsils; 8 , part of the epiglottis; 9, median glosso-epiglottidean fold (frænum epiglottidis) (from Sappey).

pharyngeal nerve is the special nerve of taste, and is distributed to the posterior third of the organ; the chorda tympani supplies the anterior two-thirds. The twelfth, 
or hypoglossal nerve, is the motor nerve of the tongue. It also helps to bring the tongue, fauces, esophagus, and larynx together, which is necessary in order to get the full effect of taste.

The mucous membrane covering the under surface of the tongue is exactly like that lining the remainder of the oral cavity. The mucous membrane upon the dorsum of the tongue, instead of being thin and smooth, is thick and rough due to the presence of papille. These structures are papillary projections of the corium and are of three varieties. The largest or circumvallate papilla, from eight to ten in number, form an inverted $\mathrm{V}$ at the back of the tongue (Fig. I40). The fungiform papille are found chiefly at the tip and side of the tongue. The filiform papille are distributed over the anterior two-thirds of the dorsum.

\section{THE ORGANS OF SMELL}

The sense of smell is located in the mucous membrane of the nose. To understand fully the sense of smell, a knowledge of the structure of the nasal cavity and the distribution of the olfactory nerve is necessary.

The nose is composed of bones, fibrocartilages, mucous membrane, and an outer covering of integument. The bones that make up the nose are the nasal and the nasal processes of the upper jaw (superior maxillary). The fibrocartilages are five in number, and give form and stability by their elasticity to the framework of the nose, at the same time guarding against injury. The mucous membrane, which lines the interior of the nose, is continuous with the skin externally and with the lining membrane of the nasal fossæ. The margins of the nostrils are provided with numerous hairs that serve to protect the delicate membrane of the nose against the entrance of irritating substances. 
The nasal fossæ are two irregular cavities extending upward and backward from the nostrils or anterior nares,

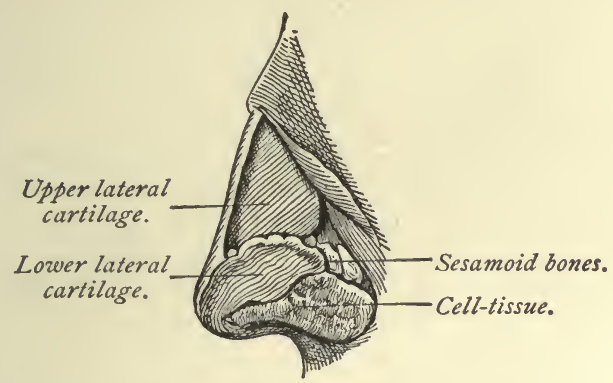

FIG. I4I.-Lateral cartilages of the nose (Ingals).

terminating in the pharynx as the posterior nares. They are bounded superiorly by the sphenoid and ethmoid

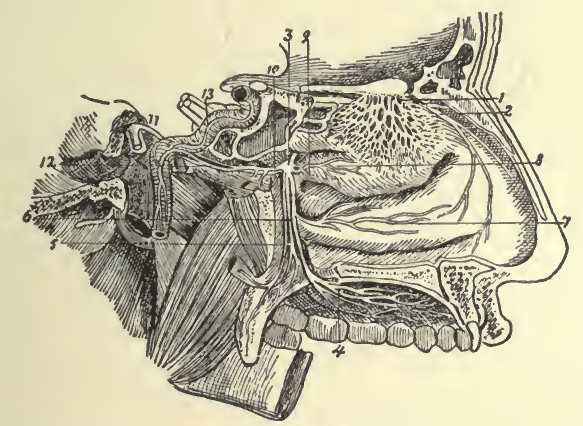

FIG. 142.-Nerves of nose and sphenopalatine ganglion, from inner side: I, Network, of external branches of olfactory nerve; 2 , nasal nerve, giving its external branch to outer wall of nose; the septal branch is cut short; 3 , sphenopalatine ganglion; 4, ramification of large palatine nerve; 5, small, and 6 , external palatine nerve; 7 , inferior nasal branch; 8 , superior nasal branch; 9, nasopalatine nerve cut short; Io, Vidian nerve; II, great superficial petrosal nerve; 2 $_{2}$, great deep petrosal nerve; ${ }_{3}$, the sympathetic nerves ascending on internal carotid artery.

bones; inferiorly, by the hard palate. In the middle line they are separated from each other by a bony and fibro- 
cartilaginous septum; upon the outer walls of each fossa, in the dried skull, are three projecting processes, termed spongy or turbinated bones. In the fresh fossa these are covered by a mucous membrane.

The space that intervenes between the superior and middle spongy bones is termed the superior meatus, or channel; the space between the middle and inferior bones, the middle meatus; and that between the inferior bone and the floor of the fossa, the inferior meatus. The meatuses are passages that extend backward from the nostrils; they contain several openings. They are lined by a mucous membrane, called the pituitary, or Schneiderian membrane (from Schneider, who first showed that the secretion of the nasal fossæ proceeded from the mucous membrane, and not from the brain, as was formerly taught).

Upon the mucous membrane of the nasal passage the olfactory nerve and also branches from other nerves ramify. This membrane is quite extensive in man, and is even more so in those animals whose sense of smell is very acute.

\section{THE ORGANS OF TOUCH}

The sense of touch or palpable feeling is the one by which the mind becomes acquainted with some of the properties of bodies; thus, it enables us to determine whether their surfaces are smooth or rough, their relative temperature, and, to a certain degree, also their form and weight. The skin, which is the principal seat of the sense of touch, has been described in a previous chapter.

Some physiologists make a distinction between the sense of touch and of tact. Tact, or feeling, is a more general faculty, extending over the whole surface of the skin and mucous membranes, whereas touch is confined chiefly to the fingers of man and the noses of quadrupeds. Tact 
is considered a passive function; for example, when any part of the system comes into contact with another body, a sensation of its presence is given without the exercise of volition. On the other hand, touch is an active sense, and is exercised voluntarily, for the purpose of conveying to the mind a knowledge of the qualities or properties of the surfaces of bodies; as, for example, when we feel a piece of cloth to ascertain its quality, or a polished surface to prove its smoothness.

In man the hand is admirably adapted for the exercise of the sense of touch. The fineness of the skin, its great sensibility, the cushion-like pad formed by the subcutaneous fat at the extremities of the fingers, the length and flexibility of these members, and the power we possess of opposing the thumb to the fingers, thus forming, as it were, a pair of forceps, are properties that are essential to the delicacy of touch, and that enable us to appreciate with exactitude the qualities of the bodies we may feel.

\section{REVIEW QUESTIONS}

What constitute the organs of sight?

How many coats has the eye? Name them.

What is the conjunctiva?

What is the sclerotic coat?

Give a description of the cornea.

What is the choroid coat?

What is the iris?

Give an illustration of the ciliary processes.

What and where is the retina?

What nerve pierces the eyeball posteriorly?

Name the different humors of the eye.

What is their function?

What are the appendages of the eye?

What are the functions of the eyebrows?

What is the function of the eyelids?

What do you understand by canthus?

What denotes the entrance to the lacrimal canal?

What is the function of the tarsal cartilages? 
What is the function of the Meibomian glands?

What is the caruncula lacrimalis?

Describe the nasal duct.

What condition is liable to ensue from obstruction of this duct?

What are the organs of hearing?

How is the sound conveyed?

How is the ear divided?

What composes the external ear?

What is the construction of the meatus auditorius?

Describe the membrana tympani.

What is the tympanum?

What are the mastoid cells? Where are they located?

What is the function of the Eustachian tubes?

- Describe the internal ear.

What are the organs of taste?

What are papillæ?

Name the varieties and their situation.

How are the papillæ on the dorsum of the tongue classified?

What is considered the nerve of taste?

What are the organs of the sense of smell?

Of what is their structure composed?

How many fibrocartilages enter into the formation of the nose?

What cavities are formed by these structures?

Where is the septum located?

What nerve supplies the sense of smell?

What do you mean by the sense of touch?

What nerve structures enter into this function?

What is the difference between tact and touch?

Where is the sense of touch situated in animals? 


\section{HA P TER XI}

\section{THE FEMALE ORGANS OF GENERATION}

\section{THE external organs of generation in the female}

are the mons veneris, labia majora, labia minora, clitoris,

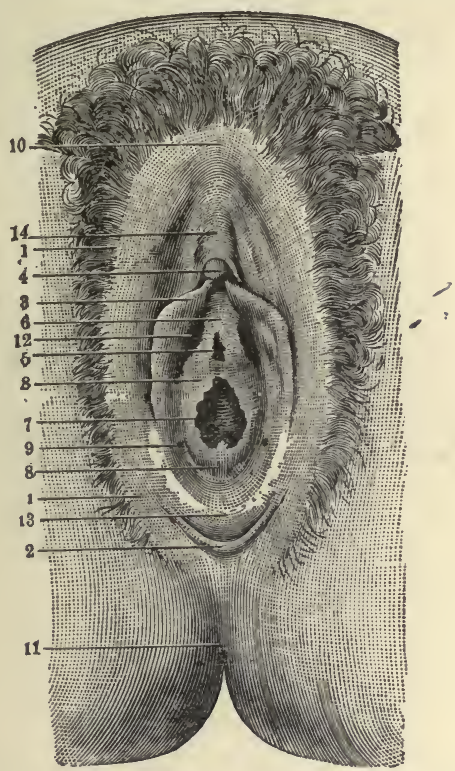

FIG. 143.-Virginal vulva : I, Labia majora ; 2, fourchet ; 3, labia minora ; 4 , glans clitoridis; 5 , meatus urinarius; 6 , vestibule; 7 , entrance to the vagina; 8 , hymen; 9 , orifice of Bartholin's gland; Io, anterior commissure of labia majora; II, anus ; I2, blind recess ; I3, fossa navicularis ; I4, body of clitoris (modified from Tarnier).

meatus urinarius, and the orifice of the vagina. The term vulva or pudendum, as generally applied, includes all these parts. 
The mons veneris is the rounded eminence situated in front of the pubes; it is formed by an accumulation of fatty tissue beneath the integument. It surmounts the vulva, and at puberty becomes covered with hair.

The labia majora are two prominent longitudinal cutaneous folds, extending downward from the mons veneris to the anterior boundary of the perineum, and enclosing an elliptic fissure, the urinosexual opening. The outer

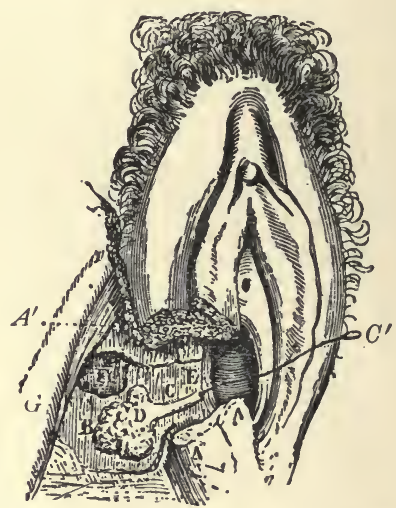

FIG. r44.- Normal vulvovaginal gland. The labium majus and minus, the sphincter vaginæ muscle, and the bulb have been partly removed on the right side in order to expose the gland: $A A^{\prime}$, Section of labium majus and minus; $B$, gland; $C$, excretory duct; $C^{\prime}$, stylet introduced into the duct; $D$, glandular end of duct; $E$, free end of duct ; $F$, section of bulb; $G$, ascending ramus of ischium (Huguier).

surfaces of the labia majora are covered with hair; the inner surfaces are continuous with those of the genitourinary mucous tract.

The labia minora are two small cutaneous folds situated within the labia majora, and extending from the clitoris obliquely downward and outward for about $\mathrm{i} \frac{1}{2}$ inches on each side of the orifice of the vagina, at the sides of which they are obliterated. They are continuous exter- 
nally with the labia majora, and internally with the vagina.

The clitoris is an erectile structure, situated beneath the anterior commissure, and partially hidden between the anterior extremities of the labia minora.

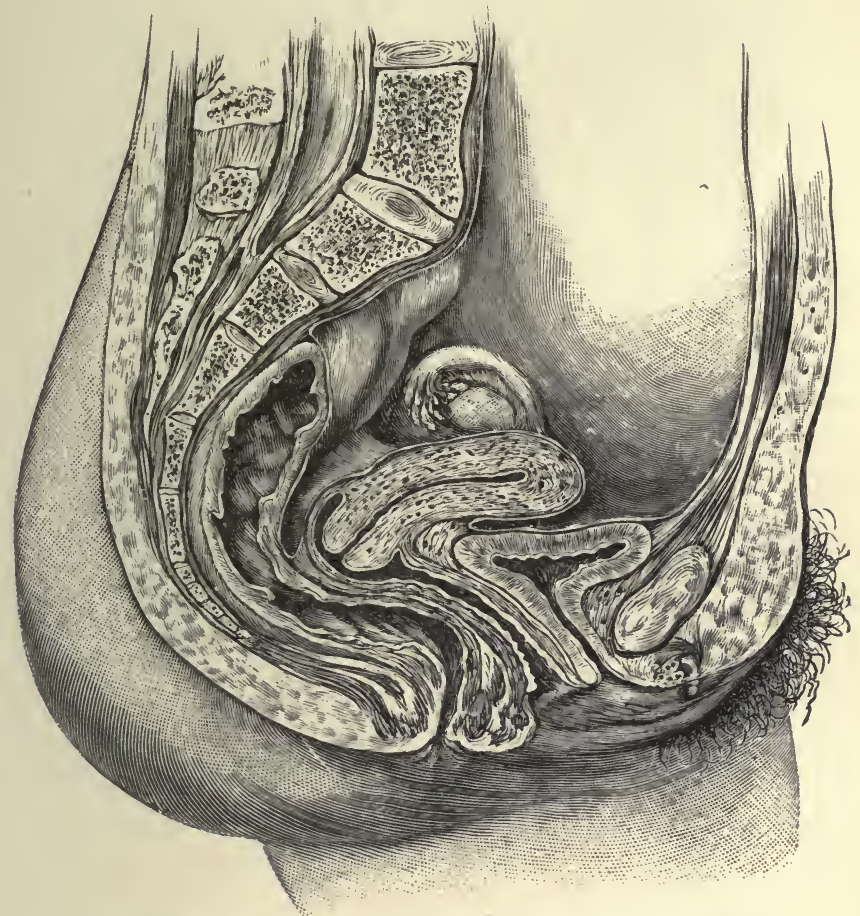

FIG. I45.-Sagittal section of the female pelvis (Dickinson).

The hymen is a thin, semilunar fold of mucous membrane, stretched across the lower part of the orifice of the vagina; its concave margin turns upward toward the pubes. Occasionally this membrane forms a complete septum across the orifice of the vagina, forming the con- 
dition known as imperforate hymen. At times it is cribriform, or it may be entirely absent. The presence of the hymen cannot, consequently, be considered as an absolute test of virginity. Its rupture, or a rudimentary condition of this membrane, gives rise to irregular elevations that surround the opening of the vagina.

The Glands of Bartholin.- These are situated one on each side of the commencement of the vagina. They are round or oblong bodies, of a reddish-yellow color, and the size of a bean; each gland opens by means of a long single duct, upon the inner side of the nymphæ, external to the hymen.

The bladder, because of its proximity to the organs of generation, is mentioned here. It is situated at the anterior part of the pelvis, and is in relation, in front, with the os pubis; behind, with the uterus, some convolutions of the small intestine being interposed; its base lies in contact with the neck of the uterus and with the anterior wall of the vagina. The bladder is larger in the female than in the male, and it is very broad in its transverse diameter. A more exhaustive description of this organ has been given in a previous chapter.

\section{THE VAGINA}

The vagina is a membranous canal extending from the vulva to the uterus. It is situated in the cavity of the pelvis, behind the bladder, and in front of the rectum. In direction it is curved forward and downward, following, at first, the line of the axis of the cavity of the pelvis, and afterward that of the outlet. It is cylindric in shape, flattened from before backward, and its walls are ordinarily in contact with each other. Its length is about 4 inches along its anterior wall. At its commencement it is constricted, becoming dilated near the uterine extremity. It surrounds the cervical portion of the cervix uteri, a 
short distance from the os, and its attachment extends higher up on the posterior than on the anterior wall. The

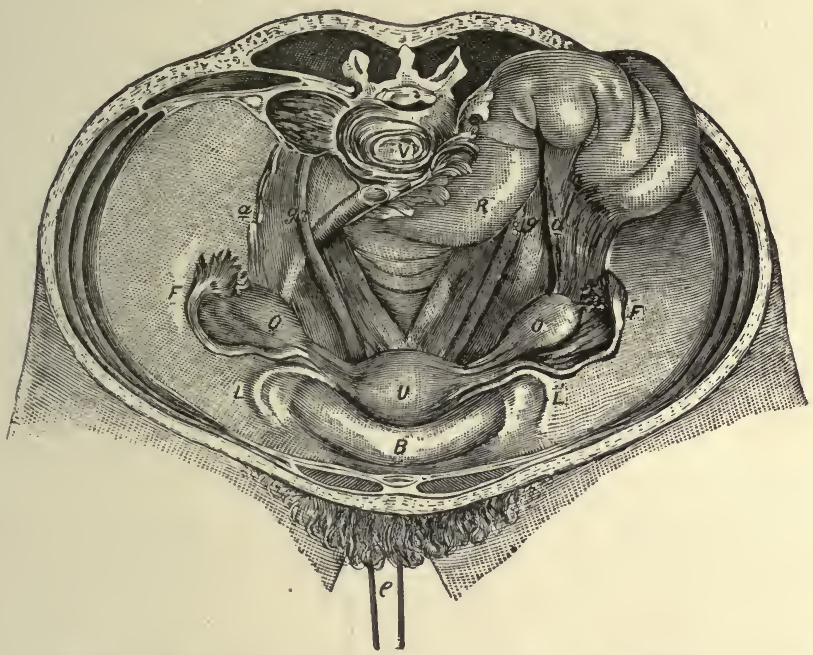

FIG. I46.-View of the pelvis and its organs: $B$, Bladder; $U$, uterus (drawn down by loop $e$ ); $F$, Fallopian tubes; $O$, ovaries; $L$, round ligaments : $g$, ureter; $a$, ovarian vessels, often prominent under their peritoneal covering (Savage).

vagina consists of a muscular coat, a layer of erectile tissue, and an internal mucous lining.

\section{THE UTERUS}

The uterus is the organ of gestation, receiving the fecundated ovum in its cavity, retaining and supporting it during the development of the fetus, and becoming the principal agent in its expulsion at the time of parturition.

In the virgin state it is pear-shaped, flattened from before backward, and situated in the cavity of the pelvis, between the bladder and the rectum; it is retained in position by the round and broad ligaments on each side, 
and projects into the upper end of the vagina below. The base projects upward and the cervix downward, in the line of the axis of the inlet of the pelvis. The uterus measures about 3 inches in length, 2 in breadth at its upper part, and I inch in thickness; it weighs from I to $I \frac{1}{2}$ ounces. For descriptive purposes it is divided into three parts-the cervix, the body, and the fundus. The fundus is the upper broad extremity of the organ; the body gradually extends toward the cervix, which is the

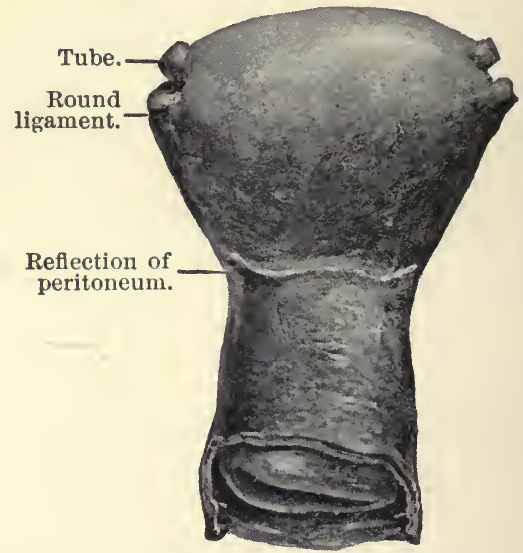

FIG. 147.-Anterior view of virgin uterus, showing relations of cervix to corpus uteri and relation of peritoneum at isthmus (The American Textbook of Obstetrics).

lower part, and is divided into two sections-the supravaginal portion above and the vaginal portion below. The vaginal cervix is consequently that part of the cervix which projects into the vagina.

Structure.-The uterus is composed of three coats - the external serous coat, a middle or muscular layer, and an internal mucous coat; the muscular coat forms the greater bulk of the organ. In an unimpregnated state it is firm, of a grayish color, and cuts almost like 
cartilage. It is classed as an involuntary muscle, and in the impregnated state the muscular tissue becomes more prominently developed.

Blood=supply. - The uterus is supplied with blood from the internal iliac through the uterine artery, and from the ovarian from the aorta.

The nerves are derived from the inferior hypogastric and spermatic plexuses, and from the third and fourth sacral nerves.

The form, size, and situation of the uterus varies at different periods of life and under different conditions.

In the fetus the uterus is contained in the abdominal cavity, projecting beyond the brim of the pelvis. The cervix is considerably larger than the body.

At puberty the uterus is pyriform in shape, and weighs from 8 to ro drams. It has descended into the pelvis, the fundus being just below the level of the brim of this cavity.

During and after menstruation this organ is enlarged, more vascular, and its surface rounder; the external os is rounded, its lips are swollen, and the lining membrane of the body is thickened, softer, and of a darker color.

During pregnancy the uterus increases in weight from $\mathrm{I} \frac{1}{2}$ to 3 pounds. It becomes enormously enlarged, and projects into the hypogastric and lower part of the umbilical region. The enlargement, which continues up to the sixth month of gestation, is due partly to increased development of pre-existing and newly formed muscular tissue. The round ligaments are enlarged, and the broad ligaments become encroached upon by the uterus making its way between the laminæ. The mucous membrane becomes more vascular, and its mucous follicles and glands enlarge; the rugæ and folds of the cervix become obliterated; the blood-vessels and lymphatics, as well as the nerves, become greatly enlarged.

After parturition the uterus almost regains its usual 
size; its weight now is from 2 to 3 ounces, but its cavity is larger than in the virgin state; the external orifice is more marked, and assumes a transverse direction; its edges present a fissured surface; its vessels are tortuous and its muscular layers are more clearly defined.

In old age the uterus becomes atrophied, paler, and denser in texture, and a more distinct constriction separates the body and cervix. The internal os and, occasionally,

A

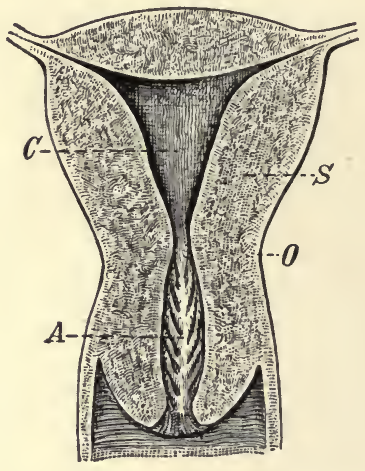

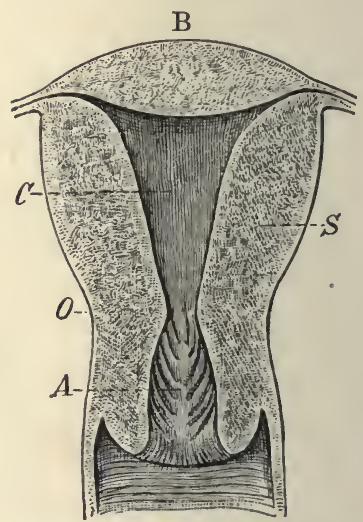

FIG. I48.-Longitudinal section of a nulliparous (A) and of a multiparous (B) uterus: $A$, Cavity of the cervix and arbor vitæ; $C$, cavity of the body ; $O$, constriction between body and cervix, the internal os; $S$, wall of body (Tarnier).

the vaginal orifice often become obliterated, and its lips almost entirely disappear.

The cavity of the uterus continues to the fundus. It is of an irregular size, a constriction at the cervical portion forming the internal os, and the part within the vagina the external os. The cavity is continuous with that of the Fallopian tubes.

The ligaments are six in number-two posterior, two anterior, and two lateral or broad ligaments. These are all formed of peritoneum. 


\section{APPENDAGES OF THE UTERUS}

The appendages of the uterus are the Fallopian tubes, the ovaries and their ligaments, and the round ligaments. These structures, together with their nutrient vessels and nerves and some scattered muscular fibers, are enclosed between the two folds of peritoneum which constitute the broad ligaments. They are placed in the following order: in front is the round ligament; the Fallopian tube occupies

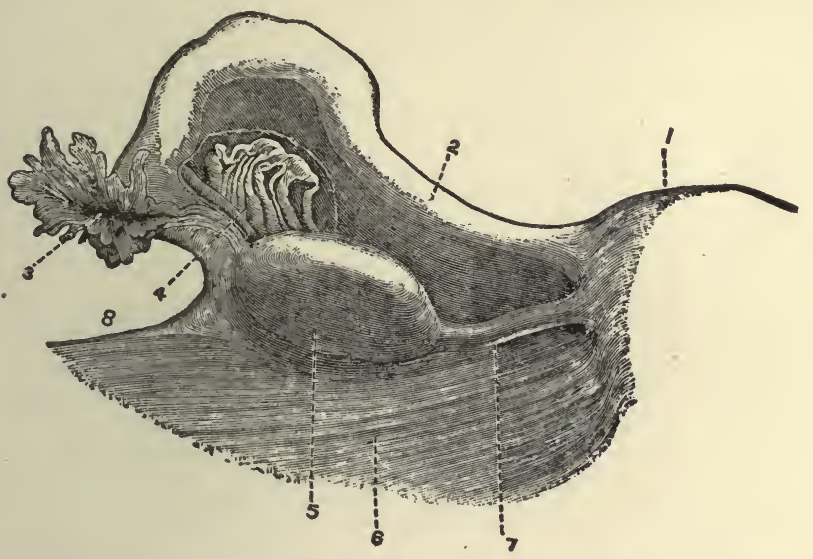

FIG. 149.-Posterior view of left uterine appendages: I, Uterus; 2, Fallopian tubes; 3 , fimbriated extremity and opening of the Fallopian tube; 4 , parovarium ; 5 , ovary ; 6 , broad ligament $; 7$, ovarian ligament ; 8 , infundibulopelvic ligament (Henle).

the free margin of the broad ligament; the ovary and its ligament are behind the latter.

The Fallopian tubes, or oviducts, convey the ova from the ovaries to the cavity of the uterus. They are two in number, one on each side, situated in the free margin of the broad ligament, extending from each superior angle of the uterus to the side of the pelvis. Each tube is about 4 inches in length; its canal is exceedingly small, and commences at the superior angle of the uterus by a minute 
orifice, the ostium internum; this will hardly admit a fine bristle; it continues narrow along the inner half of the tube, and then gradually widens into a trumpet-shaped extremity that becomes contracted at its termination. 'This orifice is called the ostium abdominale, and communicates with the peritoneal cavity. Its margins are surrounded by a series of fringe-like processes, termed fimbrinc; one of these processes is connected with the outer end of the ovary. The name fimbriated extremity is applied to this

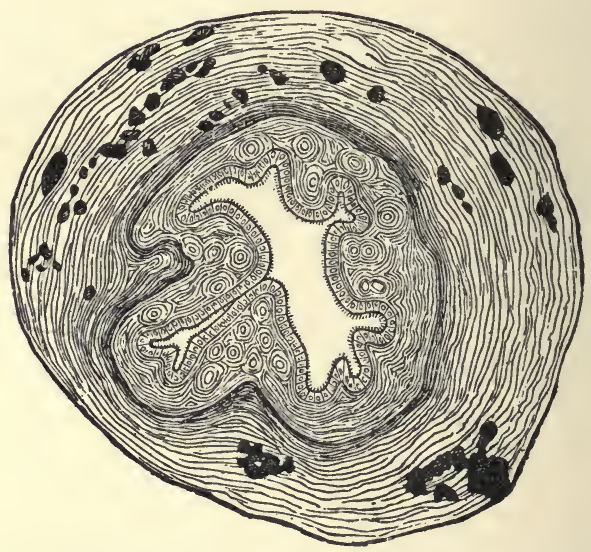

FIG. I50.-Transverse section of the human Fallopian tube (Schenck).

part of the tube; owing to the peculiar manner in which it embraces the surface of the ovary during sexual excitement it is also called morsus diaboli.

The Fallopian tubes consist of three coats-serous, muscular, and mucous.

The Ovaries. - The ovaries are oval bodies, of an elongated form, flattened from above downward, and situated one on each side of the uterus in the posterior part of the broad ligament, behind and below the Fallopian tubes. Each ovary is connected, by its anterior margin, 
with the broad ligament; by its inner extremity, to the uterus by a proper ligament-the ligament of the ovary; and by its outer end, to the fimbriated extremity of the Fallopian tube by a short ligamentous cord.

The ovaries are of whitish color, and present either a smooth or a puckered, uneven surface. They are each about $\mathrm{I} \frac{1}{2}$ inches in length, $\frac{3}{4}$ inch in width, and about $\frac{1}{3}$ inch thick; they weigh from I to 2 drams. They are attached to the broad ligament, and are invested by

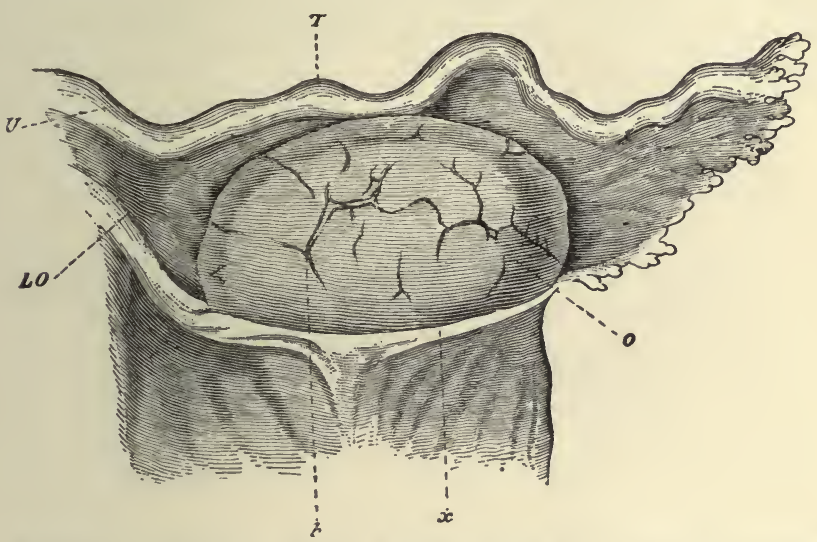

FIG. I51.-Ovary and tube of woman twenty-four years old, seen from behind: $U$, Uterus; $T$, tube ; $L O$, ovarian ligament ; 0 , ovary ; $x$, limit of peritoneum ; $b$, cicatrix after ruptured Graafian follicle (Waldeyer).

peritoneum excepting along the anterior margin, where they are attached. The ovaries contain numerous small, round, transparent vesicles in various stages of development; these are called the Graafian vesicles, the ovisacs containing the ova. In women who have not borne children they vary in number from ten to fifteen or twenty, and in size from a pin's head to a pea. It has been shown that a large number of ovisacs exist in the parenchyma of the ovary, few of which produce ova. 
These vesicles have thin, transparent walls, and are filled with a clear, colorless, albuminous fluid. The Graafian vesicles are, during their early development, small, and

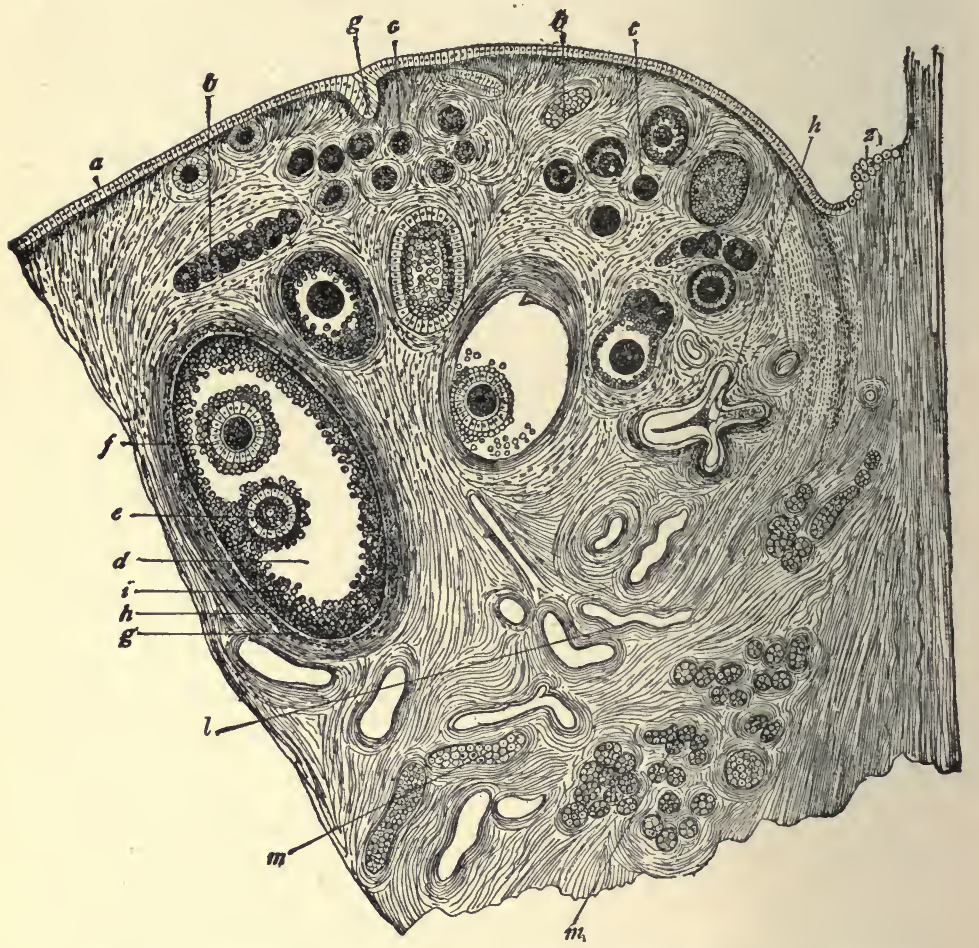

FIG. 152.-Section through part of ovary of adult bitch: $a$, Germinal epithelium; $b, b$, ingrowths (egg-tubes) from the germinal epithelium, seen in cross-section; $c, c$, young Graafian follicles in the cortical layer; $d$, a more mature follicle, containing two ova (this is rare); $e$ and $f$, ova surrounded by cells of discus proligerus; $g, h$, outer and inner capsules of the follicle; $i$, membrana granulosa; $l$, blood-vessels; $m, m$, parovarium $; g$, germinal epithelium commencing to grow in and form an egg-tube; $z$, transition from peritoneal to germinal epithelium (from Waldeyer).

deeply seated in the substance of the ovary; as they enlarge they approach the surface, and when mature they form small projections on the exterior of the ovary 
beneath the peritoneum. Each vesicle consists of an external fibrovascular coat, connected with the surrounding stroma of the ovary by a network of blood-vessels.

The ovum is an extremely minute, spheric body, measuring from $\frac{1}{200}$ to $\frac{1}{100}$ inch in diameter.

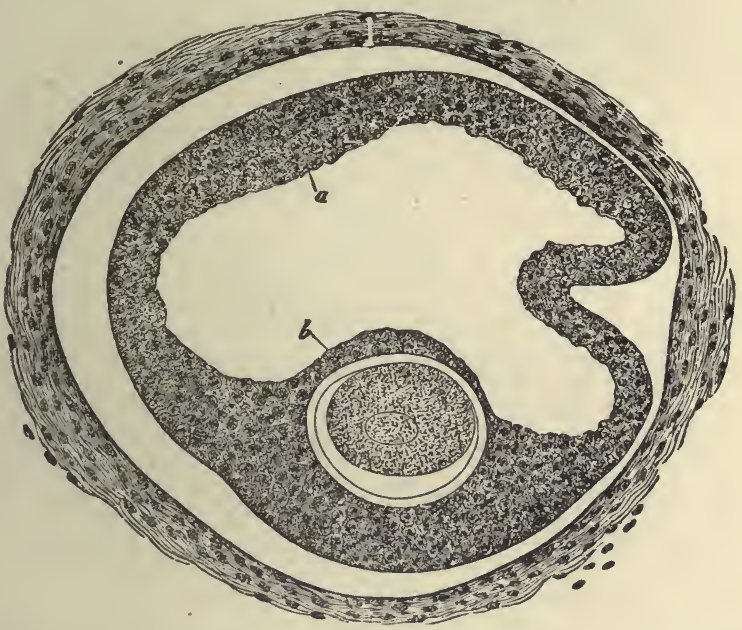

FIG. 153.-Graafian follicle from a girl seven months old: $a$, Epithelium (membrana granulosa) detached from fibrous membrane; $b$, discus proligerus, situated far away from the surface. It contains the ovum, on which the zona pellucida and the germinal vesicle are visible. The surrounding fibrous membrane is not yet separated into two layers, and there is no distinct line of demarcation between it and the surrounding stroma ( $X 220$ times; natural size, $0.35 \mathrm{I} \mathrm{mm}$. longest diameter) (Kölliker).

Discharge of the Ovum.-The Graafian vesicles, after gradually approaching the surface of the ovary, burst; the ovum and fluid contents of the vesicle are liberated and escape on the exterior of the ovary, passing thence into the Fallopian tube, the fimbriated processes of which are thought to grasp the ovary, the aperture of the tube being applied to the part corresponding to the matured and bursting vesicle. 
The maturation and discharge of the ova occur at regular periods only; these periods are known as the menstrual periods. Sexual desire is more intense in females at this time, and if union of the sexes takes place, the ovum may become fecundated. (Seè page 29i.)

The Corpus Luteum.- -Immediately after the rupture of the Graafian vesicle and the escape of its ovum the vesicle becomes filled with blood-stained fluid, and in a short time the circumference of the vesicle is occupied by a firm, yellow substance that is probably formed from plasma exuded from its walls. The exudation is at first of

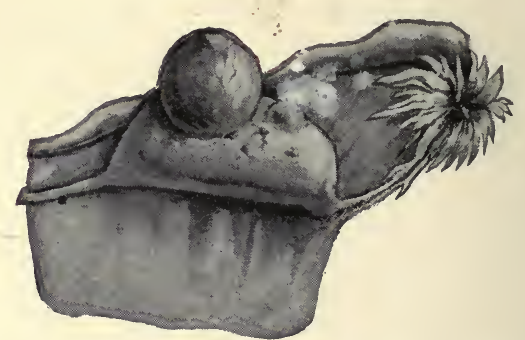

FIG. 154.-Ovary with mature Graafian follicle about ready to burst (Ribemont-Dessaignes).

a dark brown or brownish-red color, but it soon becomes paler and its consistence denser.

For every follicle in the ovary from which an ovum is discharged a corpus luteum will be found, but the character it exhibits and the changes produced in it will be determined by the circumstances of the ovum being impregnated or not.

Ligaments of the Ovaries.-The ligaments of the ovaries are rounded cords that extend from each superior angle of the uterus to the inner extremity of the ovary. They consist of fibrous tissue and a few fibers derived from the uterus. 
The round ligaments are two rounded cords, between 4 and 5 inches in length, situated between the layers of the broad ligaments, in front of and below the Fallopian tubes. Commencing on each sice at the superior angle of the uterus, each ligament pas:es forward and outward through the internal abdominal sing, along the inguinal canal, to the labia majora, in whi it becomes obliterated.

Blood-supply of the Ovaries.-The witeries of the ovaries and Fallopian tubes are the ovarian, from the aorta, anas-
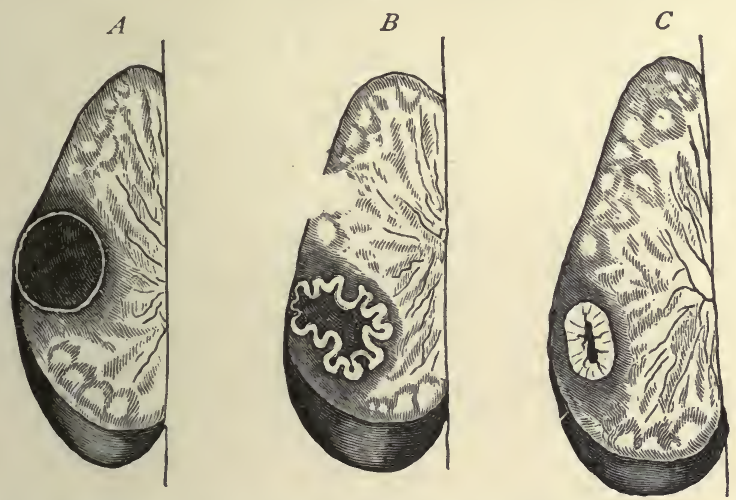

FIG. 155.-Stages in the formation of a corpus luteum: $A$, Recent blood; $B$, the wrinkling of its walls; $C$, contracting stage (A. E. Giles).

tomosing with the termination of the uterine arteries, and entering the attached border of the ovary. The veins follow the course of the arteries; they form a plexus near the ovary-the pampiniform plexus.

The nerves are derived from the spermatic plexus, the Fallopian tube receiving a branch from one of the uterine nerves.

\section{REVIEW QUESTIONS}

What are the female organs of generation?

What is the mons veneris? 
What are the labia majora? The labia minora?

What is the clitoris? The hymen?

What are the glands of Bartholin?

Describe the vagina.

What are the functions of the uterus?

What arteries supply the uterus?

State the changes that take place in the uterus at different periods of life.

What are the appendages of the uterus?

What are the Fallopian tubes?

How many are there?

What ligaments hold them in position?

What is the function of the Fallopian tubes?

What do you understand by the fimbriated processes?

What are the ovaries?

Where are they located?

What is a Graafian vesicle?

Describe an ovum.

What is the corpus luteum?

Describe the ligaments of the ovaries.

What arteries supply the ovaries? 


\section{CHA P T E R X I I}

\section{THE MALE ORGANS OF GENERATION}

THE male organs of generation include the prostate gland, the penis and the testicles, with their appendages, and the seminal vesicles.

The Prostate Gland.-The prostate gland surrounds the commencement of the urethra, lying in the pelvic cavity behind and below the symphysis pubis and in front of the rectum. It is shaped like a horse-chestnut, measuring $\mathrm{I} \frac{1}{2}$ inches transversely, $\mathrm{I}$ inch from before backward, and $\frac{3}{4}$ inch in depth. It is composed of numerous pouched glands embedded in interlacing bundles of muscular tissue, the whole being enclosed in a fibrous capsule (Fig. $156, i$ ).

The prostate gland surrounds the prostatic portion of the urethra, which is $\mathrm{I} \frac{1}{4}$ inches long. The floor of the prostatic urethra contains a longitudinal ridge, called the verumontanum or caput gallinaginis, on either side of which is a slight fossa known as the prostatic sinus. In front of the verumontanum is a depression, the sinus pocularis or uterus masculinus, upon the margin of which are the orifices of the ejaculatory ducts.

Cowper's glands are two small, rounded, yellowish bodies, about the size of peas, situated behind the beginning of the membranous portion of the urethra. They open by means of ducts on the floor of the urethra. (See Figs. I $56, h$, and 157,6 .)

The penis serves the double purpose as the termination 
of the urinary passages and as the organ of sexual intercourse. This organ consists of erectile tissue arranged in three subdivisions, two above, corpora cavernosa, and one below, corpus spongiosum, which latter surrounds the spongy portion of the urethra and terminates in the glans penis. The glans penis is covered with a loose fold of skin, the foreskin or prepuce. The corpora cavernosa are surrounded by fibro-elastic sheaths from which are given off trabeculæ. Between these trabeculæ are spaces which contain venous blood. A similar structure char-

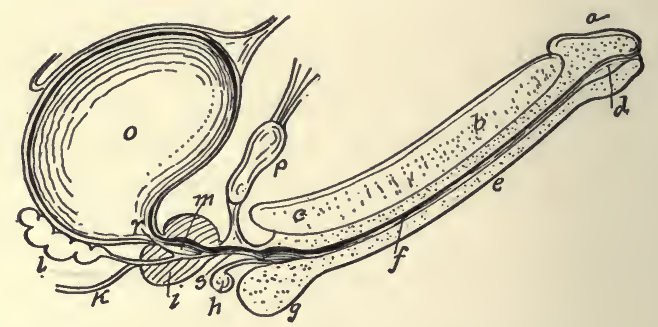

FIG. 156.-A vertical section of the bladder, penis, and urethra : $a$, Glans penis; $b, c$, corpus cavernosum; $d$, fossa navicularis ; $e$, corpus spongiosum ; $f$, spongy portion of urethra; $g$, bulb of the corpus spongiosum; $h$, Cowper's glands; $i$, the prostate; $k$, vas deferens $; l$, seminal vesicles; $m$, prostatic portion of urethra; 0 , bladder; $p$, pubic bone; $r$, commencement of urethra; $s$, membranous urethra.

acterizes the corpus spongiosum, which encloses the urethra. This structure is known as erectile tissue.

The male urethra is described on page 187 .

Testes.-The testes or testicles are the male procreating glands, which secrete the spermatozoa. They are two in number, of ovoid form, and are each suspended in the scrotum by the spermatic cord. They measure $1 \frac{1}{2}$ inches in length, I inch in breadth, and $1 \frac{1}{4}$ inches from before backward. They weigh from 6 to 8 drams each, the left being slightly the larger.

The testes are composed of lobules, the number of which 
in each organ varies from two hundred and fifty to four hundred. In each lobule are convoluted seminiferous tubules, varying in number from one to three. The coats of the testes are: the tunica vaginalis externus, an external coat derived from the peritoneum; the tunica albuginea, formed of white, fibrous tissue, surrounding the gland and reflected into its interior to form an incomplete partition from which septa are given off, separating the gland into

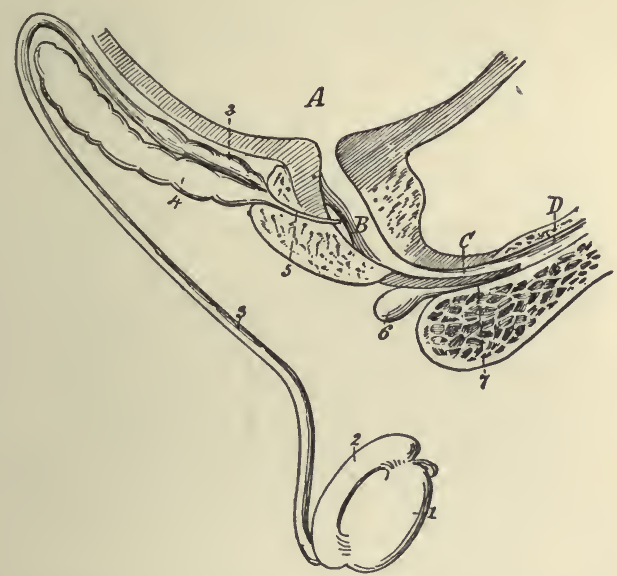

FIG. I57.-Diagram representing the male genital apparatus of right side: $A$, bladder; $B$, prostatic urethra; $C$, membranous urethra; $D$, spongy urethra; I, Right testicle; 2, epididymis; 3 , vas deferens, with 3 , its ampulla ; 4 , seminal vesicle ; 5 , ejaculatory duct opening at the verumontanum; 6 , Cowper's gland; 7 , its excretory duct (Testut).

lobes; the tunica vasculosa, or pia mater testis, consisting of a plexus of blood-vessels covering the inner surface of the tunica albuginea.

The epididymis is a convoluted tube, some 20 feet long, lying along the posterior border of the testes, being formed by a convergence of the efferent vessels of the seminiferous tubules of the testes, and terminating in the vas deferens or excretory duct of the testes. This duct, 
together with arteries and nerves from the testes, all bound together with connective tissue, forms the spermatic cord. The vas deferens passes upward from the testes through the inguinal ring and canal, then down into the pelvis and under the bladder, running forward to enter the prostatic urethra.

The scrotum is a pouch of skin and fascia suspended from the perineum and containing the testicles.

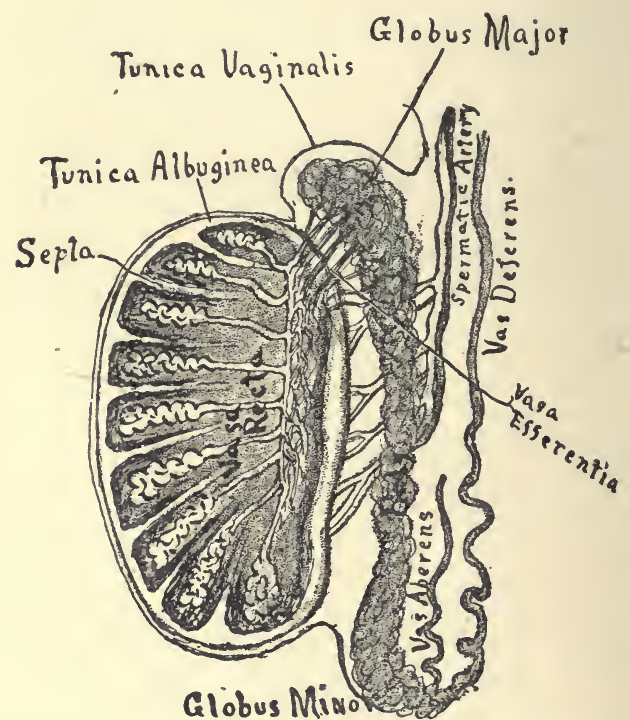

FIG. 158.-Vertical section of the testicle to show the arrangement of the ducts (Leroy).

Seminal Vesicles.-These are two lobulated receptacles for the semen which they dilute with their own secretion. They lie in contact with the base of the bladder (Figs. I59 and 160), diverging from each other from the base of the prostate to near the entrance of the ureters. They join by their anterior pointed extremities with the 
vas deferens, forming on each side the ejaculatory duct, which opens into the prostatic urethra.

Semen.-The semen, or seminal fluid, consists of secretions from the testes, seminal vesicles, prostate, and other glands connected with the male genital tract. The essential elements are the spermatozoa, or male reproductive

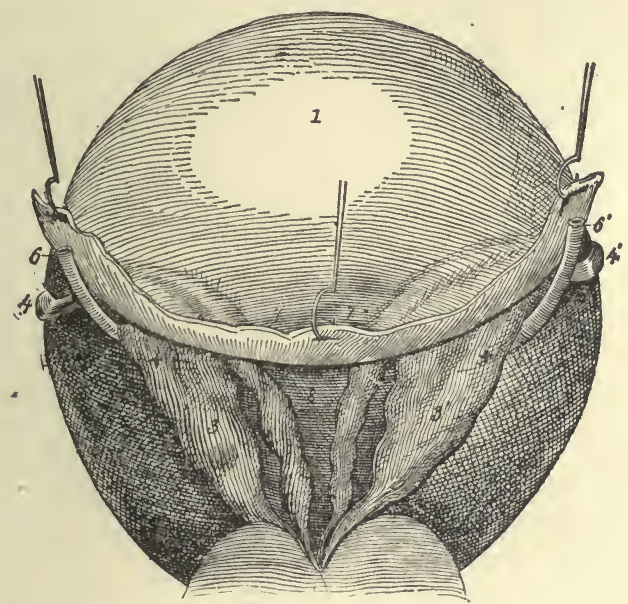

FIG. I59.-Seminal vesicles and vasa deferentia, posterior view: I, Bladder; 2 , prostate; $3,3^{\prime}$, seminal vesicles; $4,4^{\prime}$, vasa deferentia ; 5 , ejaculatory ducts $; 6,6^{\prime}$, ureters $; 7,7$, perivesicular cul-de-sac of peritoneum ; 8 , interdeferential triangle, in direct relation with the rectum, from which it is separated only by the prostatoperitoneal aponeurosis. The two crosses $(++)$ indicate the points at which the ureters disappear in the vesical wall (Testut).

cells (Fig. 16I), which are secreted by the testes. The spermatozoa are about $50 \mu$ in length and consist of a body and tail, the latter serving to give to the cell the power of moving about.

Impregnation.-The function of the spermatozoön is to produce impregnation or fertilization of the ovum. To accomplish this it must come in contact with the 
ovum in the uterus or Fallopian tube of the female. The head of the spermatozoön enters the ovum, and there

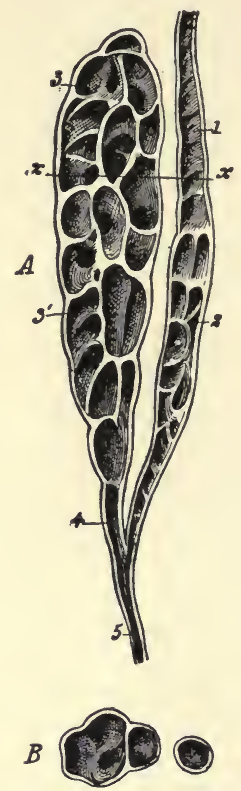

FIG. r6o.-Vas deferens and seminal vesicle, $A$, seen in longitudinal, and $B$, in horizontal, section: I, Vas deferens; 2 , its terminal or ampullary portion; 3 , seminal vesicle with $\left(3^{\prime}\right)$ its partitions; 4 , its terminal portion; 5 , ejaculatory duct (Testut).

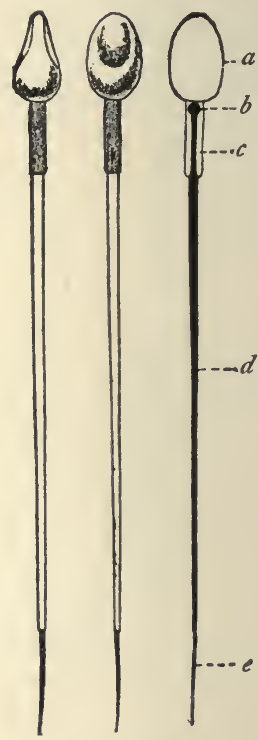

FIG. I6I.-Human spermatozoa, greatly magnified, the one at the extreme left is seen in profile; the others in surface view; the one at the right is drawn as described by Jensen : $a$, Head; $b$, terminal nodule; $c$, middle piece; $d$, tail; $e$, end-piece of Retzius (Böhm and Davidoff).

at once begins the process of cell division or segmentation, which is the first step in the development of the embryo.

\section{REVIEW QUESTIONS}

Name the male organs of generation.

What is the prostate gland? 
What relation does it have to the urethra?

What are Cowper's glands?

Describe the penis.

What is erectile tissue?

Describe the testes.

What is the tunica vaginalis?

What are the seminiferous tubules?

Describe the epididymis.

What is the vas deferens?

What is the scrotum?

Describe the seminal vesicles.

Describe the semen.

What are spermatozoa?

What is the function of the spermatozoön?

Describe the fertilization of the ovum. 


\section{CHAPTER XIII}

\section{REPAIR AND WASTE-NUTRITION-ANIMAL HEAT-PERSPIRATION-THE POWER THAT SUPPORTS AND PRESERVES HEALTH}

Having studied concisely the complex nature of man, both as regards the substances of which he is composed and the organs that go to make up his body, and having briefly pointed out the different functions of many of the organs and parts described, we will now turn our attention to a consideration of the power that keeps the organs in motion - the phenomenon that essentially constitutes life.

We have shown that each organ of the body is charged with the performance of an office or a function. Now, the performance of a function implies both an action and the power to act, for without action there could be no performance, and without the power to act there could be no action. .It is clear, therefore, that there must be a power either invested in or furnished to the organs that enables them to act. It is this power, which may be termed the living power, vital power, or power of life that we will now consider. The various terms applied to it are here used synonymously, and must always be understood as referring to that power or principle by which the vital actions are maintained and life sustained.

Waste is continually going on in our bodies; that is, the substances that make up the body are constantly being exhausted by the activity of the nervomuscular apparatus, and thus arises the necessity for our taking food. The phenomena of this waste of the substance of 294 
our tissues is a matter of interest to the general reader as well as to the nurse, and a study of the subject will afford much information that will be available to any who are seeking to acquire a knowledge of our physiologic constitution.

The necessity of supplying matter to the system arises from another necessity - that of the generation of force. It may thus be remarked that all available or active physical force, displayed in the voluntary or involuntary motions of our bodies, is derived from the force of the several affinities of the primary elements that enter into the structures.

By distributing the static state of the chemic forces power is generated. All the materials that have their affinities thus exhausted become useless in the position they occupy, and must be replaced by other or fresh materials. Here, then, is the source of waste. Thus, it may again be said, a constant interchange of material takes place in the very substance of our tissues, new material from without replacing old and effere substances that have become so in the generation of force.

It is this vital principle that prescribes the formulæ for these chemic changes, according as the involuntary actions or motions of the body may require force, or as the mind may call upon the voluntary muscles to expend force. If you reflect for a moment you will realize that you cannot act, move, or even think without demanding the sacrifice or destruction of a portion of your body equal in extent to such motion, whether it be of the mind or of the body. Thus we constantly die while we live, and in this we form a complete analogy to the entire order of organic life.

Excretion.-The materials, after having undergone the metamorphosis and having been neutralized by the readjustment of their affinities, now require to be removed 
to prevent the obstruction they would otherwise give rise to.

These materials may be compared to the ashes of a furnace-they are the result of combustion or oxidation. They are absorbed from the circulation by means, principally, of the sudorific glands, and carried off by what is called cutaneous transpiration, or perspiration. Mucous surfaces, especially those of the alimentary canal, also secrete from the circulation pecant or exhausted materials and eliminate them in the dejecta.

Pulmonary transpiration corresponds to the chimney of the furnace-it gives off the smoke or carbon dioxid and other consumed material. The kidneys remove saline and acrid products of the tissue metamorphosis that are similar to, but much more irritating than, the perspirable fluid.

\section{NUTRITION}

The processes of nutrition and the necessity for the continued supply of material have already been dwelt upon. The source of nutrition, as has been stated. elsewhere, lies in food. A question regarding nutrition that still remains unanswered is, What is the precise modus operandi of nutrition?

By the action of the various acids, salts, and other secreted products of animal combustion, which are furnished by the glandular viscera, as the liver, pancreas, and the lymphatics, the food, or ingesta, becomes admixed with these secretions in the process of digestion and the course of the circulation, and becomes still better fitted for ready combination with the substances of the tissues.

There is no force required for this nutrition, except the affinity that the several substances have for one another. It must, however, be understood that all these processes go on under vital direction of these affinities. 
These latter are not by any means of a definite form, and but for vital direction they might form very anomalous combinations.

\section{ANIMAL HEAT}

The term "animal heat" we, in common with other writers, use here merely to express the heat of the body, without attempting to distinguish one animal from another. Its generation in the system is of vast importance to health, over which it exercises a most controlling influence. It is proper, therefore, that we consider the subject somewhat at length here.

The generation of animal heat has been referred to in a previous chapter, where the agency of oxygen in the metamorphosis or change of materials composing the tissues of the organs was discussed. It may here be said that in the course of all these changes modifications are constantly taking place in the relative condition of caloric, whether sensible or latent in its state. Oxygen has never been known to combine with any other element without affecting, to some degree, the temperature of the substances concerned. In the animal body, it is true, oxidation never goes on so rapidly as in conflagrations in the open air; yet, as may be seen in the process of respiration alone, a great amount of oxygen is taken into the system, but it is breathed out again with the expired air, not, however, in the same form in which it was taken in: it is simply being mechanically received and combined with nitrogen, and given off chemically united with carbon in the form of carbon dioxid. While it is known that such a combination of oxygen and carbon cannot take place, either in or out of the body, without causing the evolution of a large amount of heat, it is by no means difficult to believe that what is called animal heat has its source in this phenomenon. It may be well to suggest that the union that occurs 
between the oxygen of the atmosphere and the blood in the lungs is one of a semimechanical character, very similar to that previously existing between the oxygen and the nitrogen in the atmosphere.

Oxygen does combine with the blood in the lungs; this is shown by the change it produces in the color and consistence of this fluid; these changes have been seen to take place in experiments exposing venous blood to the action of oxygen outside the body. It must, however, be evident that this combination occurs with the mass of the blood, and not between this element and the individual constituents of the vital fluid. In the latter case the effects must of necessity be quite different from those that take place in the lungs.

It is a fact, which appears to be self-evident, that the combustion of oxygen and carbon, whereby carbon dioxid is formed, takes place after the oxygen is carried by the circulation into the capillary vessels, for it is here that the peculiar effects of the workings of this process are evinced. It is in this part of its passage that the blood undergoes its remarkable change, and it is here that it acquires its dark purple color; it is at this time, too, that carbon dioxid, which is the evidence of combustion, or the chemic unit of oxygen and carbon, is present. If the point of origin of the process of oxidation, here said to be the cause of the evolution of animal heat, is traced, it will be found that instead of the phenomenon occurring entirely within the lungs, it takes place through the system-in every tissue and organ of the entire body.

In the circulation of the blood we find that two important changes occur; these might be said to take place at entirely opposite points. By one of these changes the color of the blood is altered to a marked red; by the other, to a dark or purple red; and it is this last-named change, and not the first, that indicates the specific office of the 
oxygen. In fact, as before stated, the agency of the oxygen is concerned in the general metamorphosis of the tisstes. In this process we at once find an evolution of heat and energy, which is the result of this phenomenon.

Heat is disseminated with more or less rapidity through all bodies, varying according to their density, but in general more easily penetrating those that are solid than those that are porous. Thus, the amount of heat is never constant, but is continually passing and repassing through matter, seeking an equilibrium or level. The human body is also subject to the same law; and as heat is constantly being generated in its tissues, it must also as constantly be eliminated; and vice versa, for the very reason that it is thus perpetually being removed, it must be continually generated. Thus, since the exercise of the day has diminished the force or quantity of the living power, the necessity arises for rest to restore it during the night. During sleep the functions are performed in a slower and more feeble manner, or cease entirely; animal heat is less rapidly evolved; the living power accumulates; the organs recover their tone, and the whole vital energies are concentrated, ready to meet the exigencies of the coming day.

\section{PERSPIRATION}

In a previous chapter we have already discussed the effects of any check to the perspiratory functions. Nevertheless, in order to make the subject more clear, we will consider it again in detail, although in doing so some repetitions will necessarily occur.

The perspiratory excretion exerts an important influence on the integrity of the living organism. A sudden check or prolonged retention of it in the body is certain to produce more or less serious derangement of the functions. There is no disease, perhaps, in which the perspiratory function is not in some measure affected. 
The perspirable fluid, or sweat, is excreted from the blood, which later, through this process, is kept in a state of purity (see, in a previous chapter, the section devoted to the appendages of the skin). As has been said elsewhere, the organs that separate the perspirable fluid from the blood are the sudorific glands; these absorb the watery poison of the blood from the capillaries or interstices of the tissues.

The perspiratory process, as has been said, is one of immense importance to the living body. Its principal uses are:

First: To moisten the external surfaces of the body. Every part of the system-the internal surfaces, even the very substance of the organs themselves, and the external skin-requires a certain degree of moisture to lubricate, soften, and qualify it for the performance of its functions. Too great a dryness of the skin injures the epidermis, or scarf-skin, and the termination of the papillæ or ends of the nerves, which impairs, and ultimately injures, the true skin and diverts sensation.

Second: To remove the worn-out material from the system. By this process the effete substances and those which are no longer useful are removed, thereby cleansing and purifying the living tissue; relieving it of a mass of morbid putrefactive matter, which, if retained in the system, would eventually interrupt the play of the organs, prove a source of irritation to their structure, and undoubtedly cause disease. By this process the blood and all the other fluids are purified, and kept in a condition most conducive to sound health. The perspiratory organs may justly be regarded as the principal natural outlets or emunctories for the surplus matter that is continually accumulating in the blood.

Third: To remove poisonous or other irritating, extraneous matter from the body. We have previously pointed out 
the method by which everything entering the body is removed from the system. Whatever is received into the stomach passes to the intestine, a portion going thence through the lacteals and thoracic duct into the blood; the gaseous substances, which enter the lungs, pass directly from these organs into the blood. Solid and liquid poisons usually enter the body through the mouth and stomach, and gaseous ones by way of the lungs. How, then, when a poison has penetrated the system, does nature expel it? If the poison is a liquid or a solid substance taken into the stomach, the irritation it produces or the administration of an emetic may induce vomiting, thus ejecting it before any part is absorbed, and so saving the system from further ill effects. But if the irritant be a gas taken into the lungs, or if the poison has had time to enter the circulation before vomiting takes place, it must then be removed from the system, through the grand emunctories of the blood-the perspiratory organs; that is, it must pass off with the perspiration. But for this most admirable provision of nature to cleanse, to purify, to drain off extraneous matter, death would ensue.

Fourth: Another important use of the perspiration is to regulate the temperature of the body. It has already been shown that much heat is abstracted by the process of evaporation. It cannot but have been observed by every one how readily exercise is followed by perspiration. This, therefore, is nature's method of regulating the heat of our bodies and thus dissipating fevers.

Failure on the part of the perspiratory organs to perform, in any degree, their functions causes a retention in the system of matters whose presence is exceedingly injurious, for putrefaction is apt to occur, whereby all the fluids of the body will become contaminated, their stimulating qualities weakened, and all the secretions so neces- 
sary to maintain the vital actions of the system will become vitiated.

When the function of perspiration is impaired, good health can never be enjoyed; hence the propriety of washing, bathing, frictions, to soften and relax the skin, in which are situated the organs that separate the perspiratory fluid from the blood.

\section{THE POWER THAT SUPPOR'TS AND PRESERVES HEALTH}

It must be evident to the reader who has carefully perused the foregoing pages that the preserving power of health can be nothing more than the living power of the system exercising its full and wholesome influence over the vital organs, by which they are kept in a healthy condition. This power, although depending upon matter exterior to the body, may be said to be exerted internally; conjointly with which are many causes having an influence upon health that depend upon the reasoning faculties ard the influence of the will. These are, principally, a suitable degree of exercise and rest; the rational indulgence of the propensities and passions; and, in short, the due observance of temperance in everything that is capable of producing either a moral or a physical effect upon the system.

\section{REVIEW QUESTIONS}

What are waste products?

Through what channels do they pass out of the system?

What means are required to supply waste?

What conditions exist when the body becomes fatigued?

What waste products are cast off from the lungs?

What waste products pass out through the kidneys?

Does the digestive tract enter into the formation of waste products? What is nutrition?

What organs are concerned in sustaining nutrition? 
Has affinity any power in its production?

What is regarded as animal heat?

How is heat generated in the body?

What takes place when oxygen is combined with carbon?

Where does the blood take on its dark color?

What two changes take place in the blood in one revolution of the same?

With these changes, what supply is provided to the system?

Why does the system require rest? .

What takes place in the system while we sleep?

What is produced when free perspiration is brought about?

Should there be a blocking-up of the perspiration? What would follow?

When poison is taken into the system, why is the skin one of the important routes of elimination?

If the poison remains in the system for a great length of time, what is the best means to eliminate it?

Is the temperature of the body influenced by perspiration?

What are required for the maintenance of good health?

\section{TABLE OF PHYSIOLOGIC CONSTANTS.-(Brubaker.)}

Mean height of malc: 5 feet $6 \frac{1}{2}$ inches; of femalc: 5 feet 2 inches. Mean weight of male: 145 pounds; of female: I 21 pounds.

Number of chemic elements in the human body: From 16 to I8.

Number of proximate principles in the human body: About Ioo. A mount of water in a body weighing 145 pounds: 108 pounds. A mount of solids in a body weighing 145 pounds: 36 pounds. A mount of saliva secreted in twenty-four hours: About $3 \frac{1}{2}$ pounds. Function of saliva: Converts starch into maltose.

Active principle of saliva: Ptyalin. A mount of gastric juice secreted in twenty-four hours: From 8 to I4 pounds. Function of gastric juice: Converts albumin into peptone.' Active principles of gastric juice: Pepsin and hydrochloric acid. Duration of digestion: From three to five hours. A mount of intestinal juice secreted in twenty-four hours: About I pounả. Function of intestinal juice: Converts starch into maltose. A mount of pancreatic juice secreted in twenty-four hours: About $\mathrm{I} \frac{1}{2}$ pounds. Active principles of pancreatic juice: Trypsin, amylopsin, and steapsin. Functions of pancreatic juice: I. Emulsifies fats. 2. Converts albumin into peptone. 3. Converts starch into maltose.

Amount of bile poured into the intestines daily: About $2 \frac{1}{2}$ pounds. 
Functions of bile: 1. Assists in the emulsification of fats. 2. Stimulates the peristaltic movements. 3. Prevents putrefactive changes in the food. 4. Promotes the absorption of the fat.

Amount of blood in the body: From 16 to 18 pounds.

Size of red corpuscles: $\frac{1}{2} \frac{1}{00}$ of an inch.

Size of white corpuscles: $\frac{1}{2500}$ inch.

Shape of red corpuscles: Circular biconcave disks.

Shape of white corpuscles: Globular.

Number of red corpuscles in a cubic millimeter (the cubic $\frac{1}{25}$ inch of blood): $5, \infty 00, \infty 00$.

Function of red corpuscles: To carry oxygen from the lungs to the tissues. Frequency of the heart's pulsation a minute: 72 on the average.

Velocity of the blood movement in the arteries: About $\mathrm{I} 2$ inches a second.

Length of time required for the blood to make an entire circuit of the vascular system: About twenty seconds.

Amount of air passing in and out of the lungs at each respiratory act: From 20 to 30 cubic inches.

Amount of air that can be taken into the lungs on a forced inspiration: ro cubic inches.

Amount of reserve air in the lungs after an ordinary expiration: Iro cubic inches.

Amount of residual air always remaining in the lungs: About too cubic inches.

Vital capacity of the lungs: About 250 cubic inches.

Entire volume of air passing in and out of the lungs in twenty-four hours: About 400 cubic feet.

Composition of air: Nitrogen, 79.19; oxygen, 20.81, in Ioo parts. Amount of oxygen absorbed in twenty-four hours: 18 cubic feet.

Amount of carbonic acid exhaled in twenty-four hours: I4 cubic feet.

Temperature of the human body at the surface: $98.6^{\circ}$ Fahrenheit.

Amount of urine excreted daily: From 40 to 50 ounces.

Amount of urea excreted daily: $5^{\mathrm{r}} 2$ grains.

Specific gravity of urine: From I.OI 5 to 1.025 .

Number of spinal nerves: $3 \mathrm{I}$ pairs.

Number of roots of origin: Two-first, anterior, efferent; second, posterior, afferent.

Rate of transmission of nerve force: About roo feet a second.

Number of cranial nerves: Twelve pairs.

Nerves of special sense: I. Olfactory, or first pair. 2. Optic, or second pair. 3. Auditory, or eighth pair. 4. Chorda tympani for anterior two-thirds of tongue. 5. Branches of glossopharyngeal, or eighth pair, for posterior one-third of tongue. 
Motor nerves to eyeball and accessory structures: Motor oculi, or third pair; pathetic, or fourth pair; abducens, or sixth pair.

Motor nerve to facial muscles: Portio dura, facial, or seventh pair.

Motor nerve to tongue: Hypoglossal, or twelfth pair.

Sensory nerve of the face: Trifacial, or fifth pair.

Sensory nerve of the pharynx: Glossopharyngeal, or ninth pair.

Sensory nerves of the lungs, stomach, etc.: Pneumogastric, or tenth pair.

Length of spinal cord: 16 to 18 inches; weight, $1 \frac{1}{2}$ ounces.

Point of decussation of motor fibers: At the medulla oblongata.

Point of decussation of sensory fibers: Throughout the spinal cord.

Function of the anterolateral columns of the spinal cord: Transmit motor impulses from the brain to the muscles.

Functions of the posterior columns: Assist in the co-ordination of muscular movements.

Functions of the medulla oblongata: Controls the functions of insalivation, mastication, deglutition, respiration, circulation, etc.

Function of the cerebellum: Center for the co-ordination of muscular movements.

Function of the cerebrum: Center for intelligence, reason, and will.

Center for articulate language: Third frontal convolution on the left side of the cerebrum.

Number of coats to the eye: Three-first, cornea and sclerotic; second, choroid and iris; third, retina.

Function of iris: Regulates the amount of light entering the eye.

Function of crystalline lens: Refracts the rays of light so as to form an image on the retina.

Function of retina: Receives the impression of light.

Function of the membrana tympani: Receives and transmits waves of sound to the internal ear.

Function of the Eustachian tube: Regulates the passage of air into and from the middle ear.

Function of the semicircular canals: Assist in maintaining the equipoise of the body.

Function of the cochlea: Appreciates the shades and combinations of musical tones.

Size of human ovum: $\frac{1}{2} 5$ inch in diameter.

Size of spermatozoön: $\frac{1}{40}$ inch in length.

Function of placenta: Acts as a respiratory and digestive organ for the fetus.

Duration of pregnancy: Two hundred and eighty days. 



\section{INDEX}

AвDомеN, muscles of, 66 action, 67 regions of, 177

Abdominal aorta, 97, 1 I I cavity, 32

Abducens nerve, 22 I

Abductor minimi digiti muscle, 75

pollicis muscle, 75

Accommodation, 260

Acetabulum, 34

Acini of mammary glands, 247

Acromion process, 34

Adductor longus brevis, 80 magnus, 80

obliquus pollicis muscle, 75

transversus pollicis muscle, 75

Adenology, definition, 12

Adipose tissue, 47

Air-cells, I 31

Albuminoids of blood, I 35

Albuminuria, 189

Alimentary tract, 155

Alveoli, I31

Amylopsin, 172

Anabolism, I8I

Anatomic neck of humerus, 37

Anatomy, definition, I I, 12

Anconeus muscle, 74

Angiology, definition, 12

Angular artery, Ioo

Animal heat, 297

Annular ligaments, 84

Anterior annular ligament, 84
Anterior auricular artery, 102 cerebral artery, I 04, 2 I 4 chamber of eye, 256 crural nerve, 227

cutaneous nerve, 227

fontanel, 26

horns of spinal cord, 2 I 9

jugular vein, 123

meningeal artery, 104, 215

nares, 267

superior spine of ilium, 34

tibial artery, i 16

nerve, 229

ulnar vein, 124

vertebral muscles, 64

Antrum of Highmore, 28

Anus, 169

Aorta, 91, 96

abdominal, 97, I I I

arch of, 96

ascending, 97

branches of, 91, 92, 96-98

descending, 97

thoracic, 97, I 10

transverse, 97

Aponeuroses, 47, 53

Appendages of eye, 258

of skin, 194

of uterus, 279

Appendix vermiformis, I68 xiphoid, 30

Aqueous humor, 253, 256

Arachnoid, 198

membrane, 209, 2 I 7 
Arbor vitæ, 199, 2 I 3

Arch of aorta, 96 of vertebræ, 22, 23 zygomatic, 28

Areola of breasts, 246

Arm, bones of, 37 veins of, 124,125 deep, 125 superficial, I 24

Arteriæ receptaculi, I03

Arterial system, 93, 94

Arteries, 93, 94

blood in, direction of, 93

blood-supply of, 96

coats of, 95

sheath of, 96

structure of, 95

Artery, medullary, I 7

Articular cartilage, 16 eminences of bones, 20 synovial membranes, 252

Ascending aorta, 97 colon, 169 pharyngeal artery, Io2

Astigmatism, 26r

Astragalus, 4I

Atlas of spine, 23

Attollens, 57

Attrahens, 57

Auditory artery, 215

canal, 26I, 262

nerve, 222

organs, $26 \mathrm{I}$

Aurem muscles, 57

Auricle of ear, 26I

Auricles, 88, 89

Auricular artery, anterior, 102 posterior, $\mathrm{IO}_{2}$

muscles, 57

vein, posterior, 122

Axillary artery, 104, 107 glands, 233
Axillary plexus, 225 vein, 125

Axis, celiac, II 2 of spine, 23 thyroid, 107

Azygos uvulæ muscle, 62 veins, 120,128

BACK, muscles of, 64

Ball-and-socket joints, 45, 46

Bartholin glands, 274

Basilar artery, 106, 2 I4

Basilic vein, 124 median, 125

Biaxial joints, 45

Biceps flexor cubiti muscle, 7 I muscle, 82

Bicuspid valve, 89,90

Big neck, 244

Bile, 1 72, 236 function of, 172

Birth, circulatory changes at, I4I

Blackheads, 247

Bladder, 186 female, 274

Blind pouch of large intestine, I 68

Blinds of eye, 258

Blood, 13 I

albuminoids of, $\mathrm{I} 35$

alkaline reaction of, $\mathrm{I} 32$

arterial, direction of, 93

changes in, in disease, 137

circulation of, 87

clotting of, 136

color of, $13 \mathrm{I}$

composition of, $\mathrm{I} 32$

corpuscles of 132 . See also

Corpuscles.

distribution, $\mathrm{I} 32$

fibrin of, 136

fibrinogen of, 136,137 
Blood, gases of, 136

hemoglobin of, $\mathrm{I} 33$

in veins, 120

liquor sanguinis of, 132

mineral salts of, 136

paraglobulin of, 136

plasma of, 132, 135

plates, I 35

properties of, I3I

proteids of, I 35

quantity of, $\mathrm{r}_{3} 6$

reaction of, 132

serum of, 136

specific gravity of, $\mathrm{I}_{32}$

temperature of, 132

Blood-supply of kidneys, 185

Blood-vessels of bone, 17

Bones, 13

articular eminences of, 20

blood-vessels of, 17

cancellated tissue of, 13

chemical analysis of, I4

classification of, 20

composition of, 14,15

age and, 15

depressions of, 20

development of, 19

eminences of, 20

flat, 20

in rickets, $\mathrm{I} 5$

inorganic constituents of, 14,15

irregular, 20

lamellæ of, I3

line of, 20

long, 20

malar, 28

marrow of, 16

nerves of, 17

non-articular depressions of, 20

eminences of, 20

of body, 22

of cranium, 24
Bones of face, 24

of nose, 266

of skull, 24

of spine, 22

of upper extremity, 34,35

organic constituents of, I4, I5

ridge of, 20

sensibility of, 14

short, 20

spine of, 20

surfaces of, 20

temporal, 27

tubercle of, 20

tuberosity of, 20

Wormian, 22

Brachial artery, 107, 108

plexus, 225

venæ comites, 124

Brachialis anticus muscle, $7 \mathrm{I}$

Brain, 198, 209

arterial supply of, 2 I4

fornix of, 212

interior of, 2 I I

lobes of, 209, 2 I I

membranes of, 198, 209

sinuses of, 215

under surface of, 2 I I

ventricles of, 212

weight of, 209

Breast-bone, 30

Breasts, 246. See also Mammary glands.

Breathing air in lungs, 150

Bridle of tongue, 243

Bronchi, 146

in respiration, $\mathbf{I}_{5} \mathbf{I}$

Bronchial arteries, I I I

Bronchocele, 244

Bronzed skin, 190

Buccinator muscle, 59

Bulbus aortæ, 96

Bursæ, 252 
Cancellated tissue, 13

Canthi, 258

Capillaries, 94

Capillary attraction, venous circulation and, 120

Capsule of kidney, 183 of lens, 257

Caput gallinaginis, 287

Cardiac orifice of stomach, 162 veins, 129

Carotid arteries, 99 artery, external, 99 internal, 103, 214

Carpus, bones of, 38

Cartilage, 16, 43, 45 articular, 16 tarsal, 258

Caruncula lacrimalis, 259

Cauda equina, 203, $218^{\circ}$

Cecum, I68

Celiac axis, II 2

Cells, mastoid, 262

Cephalic vein, 125 median, 125

Cerebellum, 198, 199, 2 13 functions of, 206

Cerebral artery, anterior, I04, 214

middle, 214

posterior, 215 hemispheres, functions of, 206

Cerebrospinal fluid, 2 10 nerves, 230

Cerebrum, 198, 199, 2 10 convolutions of, 2 Io cortical portion of, I 99, 2 I I functions of, 206 hemispheres of, 199 function, 206 medullary portion of, 199, 211 sulci of, 2 ro

Cerumen, 262
Cervical ganglia, 230 vertebræ, 22

Cervix uteri, 276

Chest, 30

Choroid coat, 253, 254 plexuses, 2 IO, 2 I 2

Chyle, I56, I 74, 245

Chyme, I 7 I

Ciliary processès, 255

Circle of Willis, 214,215

Circular fibers of iris, 255

Circulation, changes in, at birth, I 4 I

fetal, 138

of blood, 87

portal, 130

pulmonary, I 30

" venous, I 19

Circulatory system, 87 of fetus, 138

Circumvallate papilla of tongue, 266

Clavicle, 34

Clavus, 192

Clitoris, 273

Clotting of blood, 136

Coats of stomach, 162

Coccygeal vertebræ, 22

Coccyx, 33 articulation of, 23

Cochlea, 264

Cochlear nerve, 222

Coefficient of ventilation, $15 \mathrm{I}$

Collar-bone, 34

Colles' fracture, 37

Colon, 169 ascending, 169 descending, 169 transverse, 169

Common carotid arteries, 99 iliac arteries, II 4 vein, $\mathbf{2} 28$ 
Complementary air in lungs, 150

Condyles of femur, 40

of humerus, 37

Condyloid joints, 45

Conglobate glands, 233

Conjunctiva, 259

Constants, physiologic, table of, 303-305

Constrictor muscles, 63

Convolutions of cerebrum, 2 Io

Coracobrachialis muscle, $7 \mathrm{I}$

Coracoid process, 36

Corium, I91, 192

Cornea, 253, 254

Coronary artery, 98, I1 3

Corpora cavernosa, 288 quadrigemina, 213

Corpus callosum, 199, 211,212 dentatum, 199

luteum, 284

spongiosum, 288

striatum, 213

Corpuscles, 132

red, 132,133

composition of, 133

function of, 133

white, 132, 134

Corrugator supercilii muscle, 57

Cortex of kidney, 183

Cortical portion of cerebrum, 199 , 2 II

Cowper's glands, 287

Cranial nerves, 219

Cranium, bones of, 24

Crassamentum, I32, 136

Crest of ilium, 34

of tibia, 40

Crow's beak, 36

Crural nerve, 227

anterior, 227

posterior, 228

Crureus muscle, 80
Crystalline humor, 253, 256

Cul-de-sac of large intestine, 168

Cutaneous nerve, anterior, 227

external, 227

posterior, 227

transpiration, 296, 299

DEEP femoral vein, 126

Deglutition, I6o

Deltoid muscle, 69

Dental nerve, superior, 22 I

Depressions of bones, 20

Depressor anguli oris muscle, 59

labii inferioris muscle, 59

Derma, I9I

Dermatology, definition, 12

Descending aorta, 97

colon, 169

Diabetes, 189

Diaphragm, 30, 68, I 53

Diaphragmatic muscles, 68

Digastric muscle, 6I

Digestion, 154

intestinal, $170^{\circ}$

Digestive system, 154

tract, 155

Disease, blood changes in, 137

Dorsal ganglia, 23I

interossei muscle, 76

nerves, 226

vertebræ, 22

Dorsalis hallucis artery, I 16 pedis artery, 116

Ductus arteriosus, I4I

venosus, I39, I 4I

Duodenum, 165

Dura mater, 198, 209, 216

EAR, 26I

external, 26I

internal, 264

nerves of, 264 
Ear-wax, 262

Eighth cranial nerve, 222

Ejaculatory duct, 29I

Eleventh cranial nerve, 224

Eminences of bones, 20

Endocardium, 88

Endosteum, 14, I8

Ensiform of sternum, 30

Epidemic parotitis, 242

Epidermis, 191, 192

Epididymis, 289

Epigastric artery, superior, 106 region, $\mathrm{I} 78$

Epimysium, 52

Epithelium, 191

Erectile tissue, 288

Eirythrocytes, I33 composition of, 133

function of, I33

Esophageal arteries, I I I

Esophagus, 162

Ethmoid bones, 27

Eustachian tube, 263

Excretion, bodily, 295

Excretory duct of testes, 289 system, I 80

Extensor brevis pollicis muscle, 74

carpi radialis brevior muscle, 73

longior muscle, $7 \mathrm{I}$

ulnaris muscle, 74

communis digitorum muscle, 73

indicis muscle, 74

longus digitorum muscle, 83

hallucis muscle, 83

pollicis muscle, 74

minimi digiti muscle, 73

ossis metacarpi pollicis muscle, 74

External annular ligament, 84
External auditory meatus, 261, 262

carotid artery, 99

coat of arteries, 95

cutaneous nerve, 227

ear, 26I

iliac artery, I 5

vein, 126,128

intercostal muscle, 68

jugular vein, 120,123

layer of retina, 256

musculocutaneous nerve, 229

oblique muscle, 66

plantar nerve, 228

popliteal nerve, 228

pterygoid muscle, 60

Eyeball, 253, 254

Eyebrows, 258

Eyelashes, 259

Eyelids, 258

Eyes, 253

appendages of, 258

FACE, bones of, 24

muscles of, 55

Facial artery, 100

transverse, 102

nerve, $22 \mathrm{I}$

vein, $12 \mathrm{I}$

Fallopian tubes, 279

False ribs, 3i

Falx cerebelli, 198

cerebri, 198, 199

Far-sightedness, 26I

Fasciæ, 47

Fasciculi of muscle, 52

Fat, 47

Female organs of generation, $27 \mathrm{I}$

Femoral artery, I15

muscles, $77,80,82$

nerve, 227

vein, 126 
Femoral vein, deep, 126

Femur, 38

Fenestra ovalis of ear, 264 rotunda of ear, 264

Fertilization of ovum, 29I

Fetal circulation, 138

Fetus, lungs of, 140 uterus of, 277 vascular system of, 138

Fibers of iris, 255

Fibrin of blood, 136

Fibrinogen of blood, 136

Fibrocartilages of nose, 266

Fibula, 40

Fifth cranial nerve, 220

Filiform papilla of tongue, 266

Fimbriæ of oviducts, 280

Fimbriated extremity of Fallopian tubes, 280

Fingers, bones of, 38

First cranial nerve, 219

Fissura palpebrarum, 258

Fistula, lacrimal, 260

Flat bones, 20

Flexor brevis minimi digiti muscle, 75

pollicis muscle, 75

carpi radialis muscle, 72

ulnaris muscle, 72

longus pollicis muscle, 73

profundus digitorum muscle, 73

sublimis digitorum muscle, 73

Flexure, sigmoid, 169

Floating ribs, $3 \mathrm{I}$

Fluids of eye, 253

Fontanel, anterior, 26 posterior, 26

Foot, bones of, 4I ligaments of, 44 muscles of, 79,84

Foramen magnum, 25
Foramen of Monro, 212 ovale at birth, I4I

Forearm, bones of, 36 muscles of, 72

Foreskin, 288

Fornix of brain, 212

Fossa, nasal, 267

Fourth cranial nerve, 2 I9

Fracture, Colles', 37

greenstick, 15

Potts', 40

Frenum of tongue, 243

Frontal bone, 25

vein, 12 I

Fundus of uterus, 276

Fungiform papilla of tongue, 266

GALL-BLADDER, 236, 237

Ganglia, 204, 230

Ganglion, Gasserian, 221

Ganglionic nerve, 230

Gases of blood, 136

Gasserian ganglion, 2 I I

Gastric artery, 113

glands, 164,165

juice, 162

action of, 165

composition of, 164

Gastrocnemius muscle, 83

Gemellus inferior muscle, 8I

superior muscle, 8I

Generation, female organs of, 27 I

male organs of, 287

Genesiology, definition, 12

Geniohyoglossus muscle, 62

Geniohyoid muscle, 62

Genital nerve, 227

Genitalia, female, 27 I male, 287

Genitocrural nerve, 227

Gladiolus of sternum, 30

Glands, 233 
Glands, Cowper's, 287 of Bartholin, 274 of Lieberkühn, 172

Glandular system, 233

Glans penis, 288

Glenoid cavity, 35

Gliding joints, 45,46

Glomerulus of kidney, r 83

Glossopharyngeal nerve, 222

Gluteal muscles, 8o

Gluteus maximus muscle, 80 medius muscle, $8 \mathrm{r}$ minimus muscle, $8 \mathrm{I}$

Goiter, 244

Graafian vesicles, 281, 282

Gracilis muscle, 80

Gray commissure, 219

Great sciatic nerve, 228

sympathetic nerve, 229

Greenstick fracture, 15

Gustatory nerve, 264

HAIR, 194

Hand, bones of, 37 muscles of, 74 sense of touch and, 269

Haversian canals, $14,16,17$

Head, ganglia of, 230

muscles of, 55,56

of femur, 38

of fibula, 40

of humerus, 37

veins of, $\mathrm{r} 2 \mathrm{I}$

Health, power that supports, 302 preservation of, 302

Hearing, organ of, 26I

Heart, 87

cavities of, 88

opening of, 93

valves of, 89

Heart-beat, 93

Heat, bodily, 297
Heel-bone, $4^{2}$ :

Hemoglobin, I33

Hepatic artery, II 3

Highmore, antrum of, 28

Hinge-joints, 45

Hip, muscles of, 77

Horns of spinal cord, 219

House-maids' knee, 4I

Humerus, 37

Humors of eye, 253, 256, 257

Hyaloid, 257

Hydrocephalus, 212

Hymen, 273

imperforate, 274

Hyoglossus muscle, 62

Hyoid bone, 29, 30

Hyperopia, 26I

Hypochondriac region, left, 178 right, 177

Hypogastric region, 178

Hypoglossal nerve, 224

ILEOCECAL Valve, I69

Ileum, I 66

Iliac arteries, common, I 14 muscles, 76

vein, common, $\mathrm{I} 28$ external, $\mathrm{I} 26, \mathrm{I} 28$

internal, $\mathrm{x} 28$

Iliacus muscle, 77

Iliohypogastric nerve, 226

Ilio-inguinal nerve, 226

Ilium, 34

crest of, 34

Imperforate hymen, 274

Impregnation, 29x

Incus, 262

Inferior constrictor muscle, 63

maxillary bone, 29

muscles, 59

nerve, 221

meatus of nose, 268 
Inferior mesenteric artery, I 13 thyroid veins, 124 turbinated bones, 29 vena cava, 120 , I 28

Infracostales muscle, 70 Infra-orbital nerve, 22 I Infraspinatus muscle, 70

Inguinail glands, 233 muscles, 76 region, left, 178 right, 178

Inner canthus, 258 malleolus, 40

Innominate artery, 99 veins, 120,127

Insalivation, 157

Insertion of muscles, 53

Intercostal arteries, posterior, I I I

artery, superior, 107, I I I

muscles, 68

nerves, 226

spaces, 32

Intermaxillary muscles, 59

Internal annular ligament, 84 carotid artery, I03, 2 I 4 coat of arteries, 95 ear, 264

iliac artery, 115

$$
\text { vein, } 128
$$

intercostal muscle, 68 jugular vein, I2O, 123 mammary artery; 106 maxillary artery, 102 vein, I 2 I

musculocutaneous nerve, 229 oblique muscle, 67 plantar nerve, 228 popliteal nerve, 228 pterygoid muscle, 60 saphenous vein, 120 Intervertebral foramina, 204
Intestinal digestion, 170 juice, 172

Intestines, 165 large, 165,168 small, I65 coats of, 167 lymphatics of, 170,173 villi of, 167,170 villous coat of, 167

Involuntary micturition, $x 88$ muscles, 55

Iris, 253,255

Irregular bones, 20

Ischium, 34 tuberosity of, 34

Island of Reil, 2 I 3

Jaws, bones of, 27-30 muscles of, 58,59

Jejunum, I 66

Joints, 42 ball-and-socket, 45,46 biaxial, 45 condyloid, 45 gliding, 45,46 hinge, 45 pivot, 45 polyaxial, 45 saddle, 45 uniaxial, 45

Jugular vein, anterior, $\mathbf{2} 3$ external, I 20, 123 internal, 120,123

KATABOLISM, I 8 I

Kidneys, 182 blood-supply of, 185 capsule of, 183 cortex of, 183 glomerulus of, 183 medulla of, 183 structure of, 183 
Kidneys, uriniferous tubules of, 183,184 waste products eliminated by, I 81,182

Knee, house-maids', 4I

Knee-cap, 40

Knee-joint, ligaments of, 43

LABIA majora, 272 minora, 272

Labyrinth, 264

Lacrimal fistula, 260 glands, 244

Lactation, 247

Lacteals, $\mathrm{I} 70, \mathrm{I} 73$

Lacus lachrymalis, 258

Lamellæ, I3

Large intestine, $165, \mathbf{I} 68$

Larynx, I 44

Latissimus dorsi muscle, 64

Left azygos vein, r2o common carotid artery,. 99 coronary artery, 98 hypochondriac region, 178 inguinal region, 178 lumbar region, $\mathrm{I} 78$

Leg, bones of, 38,40 muscles of, $78,79,82$ veins of, 126 deep, 126

Lens, 256 capsule of, 257

Leukocytes, I34

Leukocytosis, I 34

Levator costarum muscle, 68 labii muscle, 59 palati muscle, 62 palpebræ muscle, 57

Lieberkühn, glands of, $\mathbf{I} 72$

Ligamenta dentata, 217

Ligaments, 44 annular, 84
Ligaments of foot, 44

of knee-joint, 43

of ovaries, $28 \mathrm{o}, 28 \mathrm{I}, 284$

of shoulder, 42

of uterus, 278

of wrist-joint, 43

round, 285

sutural, 42

Ligamentum denticulatum, 203

Line of bones, 20

Linea alba, 67

Lingual artery, 100 bone, 30

Lingualis muscle, 62

Liquor sanguinis, $\mathrm{I} 32$

Liver, 235

blood-supply of, 236

color of, 236

function of, 236

Lobules of testicles, 288

Long bones, 20

Longus colli muscle, 64

Lower extremities, bones of, 38 , 39

muscles of, 76

veins of, $\mathrm{I} 26$

Lumbar arteries, II4

ganglia, $23 \mathrm{I}$

nerves, 226

plexus, 226

region, left, $\mathbf{1} 78$

right, 178

vertebræ, 22

Lumbricales muscle, 76

Lungs, 145

breathing air in, 150

color of, 146

complementary air in, 150

of fetus, 140

relations of, 146

reserve air in, I5O

residual air in, I5O 
Lungs, shape of, 146 situation of, 145 substance of, 146 supplemental air in, 150 tidal air in, 150 waste products eliminated by, I 81

weight of, 147

Lymph, 73

Lymphatic glands, 233 of small intestine, 170, 173

MALAR bones, 28

Malleolus, inner, 40

Malleus, 262

Malpighian bodies, 183

Mammary artery, internal, 106 glands, 246 acini of, 247

areola of, 246

changes in, 247

lactation, 247

structure of, 247

Manubrium of sternum, 30

Marrow, I6

Masseter muscle, 59

Mastication, 156 nerves involved in, 157

Mastoid cells, 262

Matrix of nail, 196

Maxillary artery, internal, ro2 bone, inferior, 29 superior, 27 muscles, inferior, 59 superior, 58

nerve, inferior, $22 \mathrm{I}$ superior, 221 vein, internal, I 21

Meatus, external auditory, 262 of nose, 268 urinarius, 187

Median basilic vein, 125
Median cephalic vein, 125 vein, 125

Mediastinal arteries, posterior, I I I

Mediastinum, 145,152

Medulla oblongata, 198-200, 213 functions of, 207 of kidney, 183

Medullary artery, 17 portion of cerebrum, 199, 2 II

Meibomian glands, 259

Membrana tympani, 262

Membranes of body, 25I

of brain, 198, 209

of spinal cord, 216

Meningeal arteries, 215 artery, anterior, 104, 215 middle, 215 posterior, 215

Menstrual periods, 284

Menstruation, uterus after, 277 during, 277

Mesenteric artery, inferior, I I 3 superior, II 3 glands, 233, 245

Mesentery, I65

Metabolism, I80, I8I

Metacarpus, 38

Metatarsal bones, 42

Micturition, 187 involuntary, 188

Middle cerebral artery, 214 coat of arteries, 95 constrictor muscle, 63 meatus of nose, 268 meningeal artery, 215 temporal artery, 102

Mineral salts of blood, $\mathrm{I} 36$

Mitral valve, 89,90

Mixed nerves, 208

Monro, foramen of, 2 I 2

Mons veneris, 272 
Morsus diaboli, 280

Motion, nerves of, 207

Motor oculi nerves, 219

Mucous membrane, 25 I of nose, 266

Mumps, 242

Muscles, 52

auricular, 57

contractility of, 53,54

diaphragmatic, 68

femoral, $77,80,82$

gluteal, 80

iliac, 76

inferior maxillary, 59

inguinal, 76

insertion of, 53

intermaxillary, 59

involuntary, 55

irritability of, 54

maxillary, 58

nasal, 58

non-striped, 55

number of, 53

occipital, 55

of abdomen, 66

action, 67

of back, 64

of face, 55

of foot, 79,84

of forearm, 72

of hand, 74

of head, 55,56

of hip, 77

of jaws, 58,59

of leg, $78,79,82$

of lower extremities, 76

of neck, $56,60,6 \mathrm{I}$

of nose, 58

of orbit, 57

of palate, 62

of perineum, 84,85

of thigh, 78

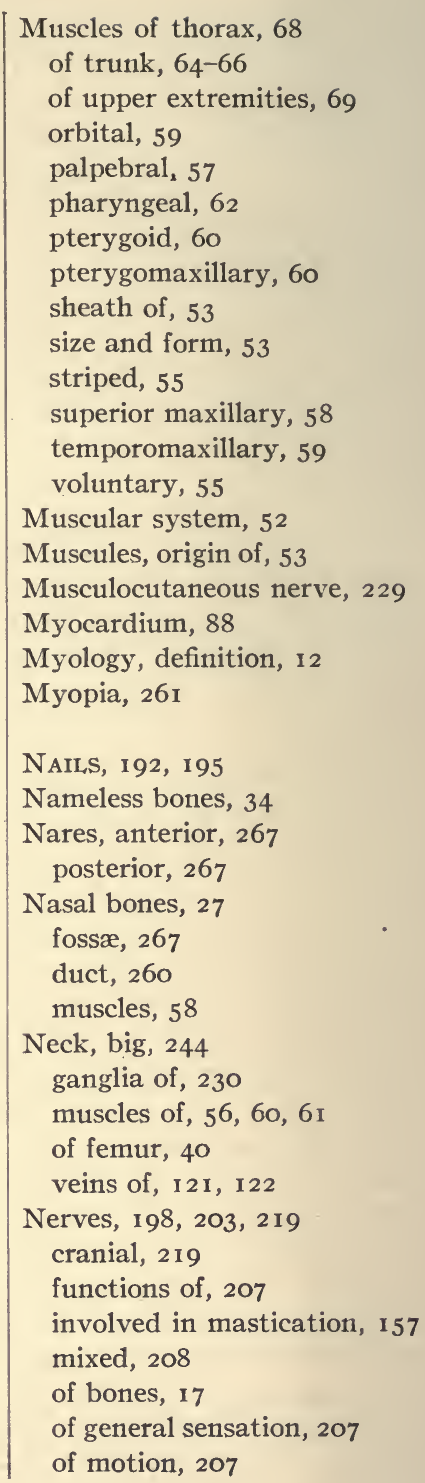


Nerves of organic life, 229 of special sensation, 207 sacral, 228 spinal, 203, 224 sympathetic, 208, 229, 230

Nervous membrane of retina, 256 system, 198

functions of, 206

Neurilemma, 203, 2 I 7

Neurology, definition, I 2

Ninth cranial nerve, 222

Nipple, 246

Non-striped muscles, 55

Nose, 266

bones of, 27,266

fibrocartilages of, 266

fossæ of, 267

meatus of, 268

mucous membrane of, 266

muscles of, 58

septum of, 268

Nostrils, 267

Nutrition, 29I

OBLique muscles, 66, 67

Obturator externus muscle, $8 \mathrm{I}$ internus muscle, $8 \mathbf{I}$ nerve, 227

Occipital artery, Ior bone, 25

muscles, 57

vein, 122

Occipitofrontalis muscle, $\mathbf{5 5}$

Ocular arteries, 104

Olecranon, 37

Olfactory nerve, 2 I 9

Olivary bodies, 2 I 4

Omohyoid muscle, 6I

Ophthalmic artery, 104 nerve, 22 I

Opponens minimi digiti muscle,
Opponens pollicis muscle, 75

Opsonin, 135

Optic lobes, 2 I 3

nerve, 2 I 9

thalamus, 2 I 2

Orbicularis oris muscle, 59 palpebrarum muscle, 57

Orbit, 29 muscles of, 57

Orbital arteries, 102, 104

Organs of sight, 253

of special sense, 253

Os calcis, 42

Ossa innominata, 23, 34

Osseous system, I3

Osteoblasts, 18,19

Osteology, I 3

definition, 12

Ostium abdominale, 280 internum, 280

Outer canthus, 258

Ovarian arteries, I I4

Ovaries, 280

arteries of, 285

blood-supply of, 285

ligaments of, 28o, 28I, 284

nerves of, 285

veins of, 285

Oviducts, 279

Ovum, 283

discharge of, 283

Oxidation, 297

bodily, 297

Oxygen carriers, I 33

Palate bones, 28 muscles, 62

Palatoglossus muscle, 62

Palatopharyngeus muscle, 62,63

Palmar interossei muscle, 76

Palmaris brevis muscle, 75

longus muscle, 72 
Palpebræ, 258

Palpebral muscles, 57

Pampiniform plexus, 285

Pancreas, 24I

Pancreatic juice, I7I, 24I

function of, I 7 I

Papillæ of eyelids, 258

of skin, 192

of tongue, 266

Paraglobulin of blood, 136

Parietal bones, 25

Parotid arteries, 102 glands, 24I

Parotitis, epidemic, 242

Parturition, uterus after, 277

Patella, 40

Pathetic nerve, 219

Pavilion of ear, 26I

Pectineus muscle, 80

Pectoralis major muscle, 69 minor muscle, 69

Pelvis, 30,32

brim of, 32

cavity of, 32

outlet of, 33

Penis, 287

Pericardiac arteries, II I

Pericardium, 88, 25I

Perimysium, 52

Perineum, 84 muscles of, 84,85

Periosteum, 14, 18

Peristalsis, 170

Peritoneum, 25I

Permanent teeth, 160

Perspiration, 296, 299 uses of, 300

Peyer's patches, 168

Phagocytes, I 35

Phagocytosis, 135

Phalanges of foot, 42 of hand, 38
Pharyngeal artery, ascending, 102

muscles, 62

Pharynx, 63

Phrenic arteries, 112

Physiologic constants, table of, 303-305

Physiology of vision, 260

Pia mater, 199, 210,217

testis, 289

Pigmentum nigrum, 255

Pinna, 26I

Pituitary membrane, 268

Pivot joints, 45

Placenta, I4I

Plantar nerve, 228

Plasma of blood, 132, I35

Platysma myoides muscle, 60

Pleura, 152, 25 I

Pleural cavity, 152

membrane, 145

Plexus, 203

axillary, 225

brachial, 225

lumbar, 226

pampiniform, 285

preaortic, 230

prostatic, 128

sacral, 228

uterine, 128

vesical, $\mathrm{I} 28$

visceral, 230

Pneumogastric nerve, 222

Polyaxial joints, 45

Pons varolii, 213

Popliteal artery, I 6

nerve, external, 228

internal, 228

vein, 126

Pores of skin, 249

Portal circulation, 130

veins, 130 
Posterior auricular artery, 102 vein, 122

cerebral arteries, 215

chamber of eye, 256

crural nerve, 228

cutaneous nerve, 227

fontanel, 26

horns of spinal cord, 2 I 9

intercostal arteries, I I I

mediastinal arteries, I I I

meningeal artery, 2 I 5

nares, 267

tibial artery, i 8

ulnar vein, 124

Potts' fracture, 40

Power of accommodation, 26I

Preaortic plexus, 230

Pregnancy, uterus during, 277

Prepuce, 288

Presbyopia, 26I

Prevertebral sympathetic nerve, 229

Profunda cervicis artery, I07

Pronator quadratus muscle, 73 teres muscle, 72

Prostate gland, 287

Prostatic plexus, 128 sinus, 287

Proteids of blood, 135

Protoplasm, r34

Psoas magnus muscle, 76 parvus muscle, 77

Pterygoid artery, 103 muscles, 60

Pterygomaxillary muscles, 6o

Ptyalin, 157

Puberty, uterus at, 277

Pubes, 34

Pudendum, 271

Pulmonary artery, 130

circulation, 130

transpiration, 296
Pulmonary veins, I3I

Pupil, 255

Pyloric orifice of stomach, 162

Pyriformis muscle, 8I

QUADRatus femoris muscle, 8I

RAchirIs, bones in, I5

Radial artery, 109

vein, 125

Radiating fibers of iris, 255

Radius, 37

Ranine artery, roo

Receptaculum chyli, I 73

Rectum, 169

Rectus capitis anticus major muscle, 64

minor muscle, 64

femoris muscle, 79

lateralis muscle, 64

Red corpuscles 132, 133

composition of, 133

function of, I 33

Reil, island of, 2 I 3

Renal arteries, I I 4

Repair of body, 294

Reserve air in lungs, 150

Residual air in lungs, I5O

Respiration, 147

accessory organs of, I5 I

air taken into lungs in, 148

bronchi in, I5I

trachea in, $15 \mathrm{I}$

Respiratory center, 152

quotient, I 49

system, I 44

Restiform bodies, 2 I 4

Retina, 256

Retrahens, 57

Ribs, 31, I 53 .

false, 3 I

floating, $3 \mathrm{I}$ 
Ribs, spaces between, 32

true, 3 I

vertebral, 3 I

vertebrocostal, 3 I

Rickets, bones in, I 5

Ridge of bones, 20

Right azygos vein, 120

common carotid artery, 99

coronary artery, 98

hypochondriac region, I 77

inguinal region, 178

lumbar region, 178

Risorius muscle, 59

Rouleaux, I34

Round ligaments, 285

SACRA media, I 14

Sacral ganglia, 23 I

nerves, 228

plexus, 228

vertebræ, 22

Sacrum, 33

Saddle joints, 45

Saliva, I 57, 24I

in mastication, 156

Salivary duct, 242

Salts, mineral, of blood, I 36

Saphenous vein, internal, I 20

long, 127

short, 127

Sartorius muscle, 78

Scalenus anticus muscle, 64 medius muscle, 64 posticus muscle, 64

Scapula, 34

Scarf-skin, I 9 I

Schneiderian membrane, 268

Sciatic nerve, 228

Sclerotic coat of eye, 253

Scrotum, 288, 290

Sebaceous glands, 192, 194, 247

Sebum, 247
Second cranial nerve, 2 I 9

Secretory glands of skin, 192

Segmentation, 292

Semen, 29I

Semicircular canals of ear, 264

Semilunar valve, $89,91,96$

Semimembranosus muscle, 82

Seminal vesicles, 290

Seminiferous tubules, 289

Semitendinosus muscle, 82

Sensation, nerves of, 207

Septum of nose, 268

Serous membranes, 25 I

Serpentine artery, I 3

Serratus magnus muscle, 69

Serum of blood, 136

Seventh cranial nerve, 22 I

Sheath of arteries, 96 of muscle, 53

Shin bone, 40

Short bones, 20

Short-sightedness, 26I

Shoulder girdle, 34

ligaments of, 42

Shoulder-blade, 34

Sight; organs of, 253

Sigmoid cavity, 37

flexure, I 69

notch, 30

Sinus pocularis, 287

prostatic, 287

Sinuses, I 2 I

of brain, 2 I 5

Sixth cranial nerve, 22 I

Skeleton, I3, 2 I

Skin, 190

appendages of, 194

bronzed, 190

glands of, 192

layers of, I9I

papillæ of, 192

pores of, 249 
Skin, scarf-, 191 secretory glands of, 192

true, 19I waste products eliminated by, $18 \mathrm{I}, 182$

Skull, 24 bones of, 24

Small intestine, 165 coats of, 167

lymphatics of, I 70,173 villi of, 167,170 villous coat of, 167

Smell, organs of, 266

Soft spot, 26

Specific gravity of blood, 132 of urine, 188

Spermatic arteries, I 14 cord, 290

Spermatozoa, 29I

Sphenoid bones, 27

Spinal accessory nerve, 224 column, 22 atlas of, 23

axis of, 23

curves of, 25

length of, 23

regions of, 22

cord, 201, 216,217

columns of, 202, 218

fissures of, 218

functions of, 207

gray commissure of, 219

horns of, 219

membranes of, 216

nerves of, 203, 224

nerves, 203, 224

veins, 129

Spine, 22. See also Spinal column.

bones of, 22

of bone, 20

of scapula, 34
Splanchnology, definition, 12

Spleen, 237

function of, 238

Splenic artery, I1 3

Spongy bones, 268

Stapes, 262

Steapsin, I7 I, I 72

Sternocleidomastoid muscle, 60

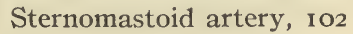

Sternothyroid muscle, 6 I

Sternum, 30

Stomach, 162

coats of, 162

openings of, 162

Striped muscles, 55

Styloglossus muscle, 62

Stylohyoid muscle, 62

Stylopharyngeus muscle, 63

Subanconeus muscle, $7 \mathrm{I}$

Subarachnoidean spaces, 2 I0

Subclavian artery, 104 vein, 120,126

Subclavius muscle, 69

Subcrureus muscle, 80

Sublingual glands, 243

Submaxillary glands, 243

Subscapularis muscle, 69

Succus entericus, 172

Sudorific glands, 192, 300

Sudoriparous glands, 249

Sulci of cerebrum, 2 I0

Supercilia, 258

Superior constrictor muscle, 63

dental nerve, $22 \mathrm{I}$

epigastric artery, 106

intercostal artery, 107, I I I

maxillary bones, 27

mi1scles, 58

nerve, $22 \mathrm{I}$

meatus of nose, 268

mesenteric artery, 113

thyroid artery, Ioo 
Superior vena cava, I 20, 127

Supinator brevis muscle, 74 longus muscle, 73

Supplemental air in lungs, 150

Suprarenal arteries, 114 capsules, 190

Supraspinatus muscle, 70

Surgical neck of humerus, 37

Sutural ligament, 42

Swallowing, 160

Sweat, 300

glands, 192, 193, 249

Sympathetic nerve, 208, 229, 230

Symphysis pubis, 34

Syndesmology, definition, I2

Synovia, 252

Synovial membrane, 44, 45, 252

TACT, 268

Tarsal cartilages, 258

Tarsus, 4I

Taste, organs of, 264

Teeth, 159

permanent, 160

temporary, 159

Tegumentary areolar tissue of eyelids, 258

Temperature of blood, 132

Temporal artery, I02 middle, I02

bones, 27

of infant, 26

muscle, 59

vein, I 2 I

Temporary teeth, $\mathbf{I} 59$

Temporomaxillary muscle, 59 vein, 121

Tendons, 46, 53

Tensor palati muscle, 62 tarsi muscle, 57 vaginæ femoris muscle, 77
Tenth cranial nerve, 222

Tentorium cerebelli, 198

Teres major muscle, 7 I minor muscle, 7 I

Testes, 288

Testicles, 288

Thigh, muscles of, 78

Thigh-bone, 38

Third cranial nerve, 219

Thoracic aorta, 97, 110

cavity, 30

duct, 175

Thorax, 30

muscles of, 68

Thread of tongue, 243

Thyrohyoid muscle, 6I

Thyroid artery, superior, 100 axis, 107

gland, 244

veins, inferior, 124

Tibia, 40

Tibial artery, anterior, I I 6 posterior, 1 i 8 nerve, anterior, 229 veins, 126

Tibialis anticus muscle, 83

Tidal air in lungs, 150

Tissue, erectile, 288

Tongue, 264 bridle of, 243

frenum of, 243 nerve supply of, 264 papillæ of, 266 thread of, 243

Torticollis, $6 \mathrm{I}$

Touch, organs of, 268

Trachea, 145,146 in respiration, $15 \mathrm{I}$

Transpiration, cutaneous, 296, 299 pulmonary, 296

Transverse aorta, 97 colon, 169 
Transverse facial artery, 102

Trapezius muscle, 64

Tree of life, 213

Triangularis sterni muscle, 68

Triceps extensor cubiti muscle, 71

Tricuspid valve, 89,90

Trifacial nerve, 220

Trisplanchnic nerve, 208

Trochanters, 40

True ribs, 3 I

skin, I9I

Trunk, muscles of, $64-66$

veins of, 127

Trypsin, 172

Tubercle of bone, 20 of eyelids, 258

Tubercula quadrigemina, 2 I 3

Tuberosity, 20 of humerus, 37

of ischium, 34

Tunica albuginea, 289

vaginalis externus, 289

vasculosa, 289

Turbinated bones, 268 inferior, 29

Twelf th cranial nerve, 224

Tympanic artery, 103 membrane, 262

Tympanum, 262

ULNA, 37

Ulnar artery, 109, I 10 vein, anterior, 124 posterior, 124

Umbilical arteries, I4I region, $\mathbf{I} 78$

veins, $138, \mathbf{1}_{4} \mathbf{I}$

Uniaxial joints, 45

Upper extremities, bones of, 34, 35

muscles of, 69

veins of, 124
Urea in urine, 189

Ureters, 186

Urethra, 187

Urine, 188

character of, 188

color of, 188

composition of, 189

constituents of, abnormal, 189

normal, i 89

quantity of, 189

reaction of, 188

transparency of, 188

urea in, 189

Uriniferous tubules of kidney, 183,184

Urinosexual opening, 272

Uterine plexuses, 128

Uterus, 275

after menstruation, 277

after parturition, 277

appendages of, 279

at puberty, 277

blood-supply of, 277

body of, 276

cavity of, 278

cervix of, 276

coats of, 276

during menstruation, 277

pregnancy, 277

fetal, 277

fundus of, 276

in old age, 278

ligaments of, 278

masculinus, 287

nerves of, 277

situation of, 277

size of, 277

structure of, 276

Uvea, 255

VAGINA, 274

Vaginal synovial membranes, 252 
Vagus nerve, 222

Valves of heart, 89 of veins, I 19

Vas deferens, 289

Vasa vasorum, 96

Vascular membrane of retina, 256 system, 87

of fetus, 138

Vastus externus muscle, 79 internus muscle, 80

Veins, 1 I 8

blood in, 120

classification of, 120

of arm, 124, 125

deep, 125

superficial, 124

of head, I 2 I

of leg, 126

deep, 126

of lower extremities, I 26

of neck, I 21,122

of trunk, 127

of upper extremities, I 24 valves of, I 19

Velum interpositum, 210, 21 2

Vena azygos major, 128

cava, inferior, I 20, 128

superior, 120,127

Venæ comites, I24

Galeni, 212

Venous circulation, I 19 system, I I 8

Ventilation, cœfficient of, 15 I

Ventricles, 88,89 of brain, 212

Vermiform appendix, $\mathbf{x} 68$

Vernix caseosa, 247

Vertebræ, 22, 23

Vertebral artery, 106, 214
Vertebral column, 22. See also Spinal column. muscles, anterior, 64 ribs, 3 I sympathetic nerve, 229 vein, 123,129

Vertebrocostal ribs, 3I

Verumontanum, 287

Vesical plexus, I 28

Vesicles, Graafian, 28I, 282 seminal, 290

Vestibular nerve, 222

Vestibule of ear, 264

Villi of small intestine, 167,170

Villous coat of small intestine, I 67

Visceral plexus, 230

Vision, physiology of, 260

Vitreous humor, 253, 257

Voluntary muscles, 55

Vomer, 27, 29

Vulva, 27 I

WASTE, bodily, 294 products, channels of elimination, 181,182 elimination of, $\mathrm{I} 8 \mathrm{I}$

Wax of ear, 262

White corpuscles, 132,134 of eye, 254

Willis, circle of, 214, 215

Windpipe, 146

Wormian bones, 22

Wrist-joint, ligaments of, 43

Wry-neck, 6I

XIPHOID appendix, 30

Zygomatic arch, 28 


DATE DUE SLIP

UNIVERSITY OF CALIFORNIA MEDICAL SCHOOL LIBRARY

THIS BOOK IS DUE ON THE IAST DATE STAMPED BELOW

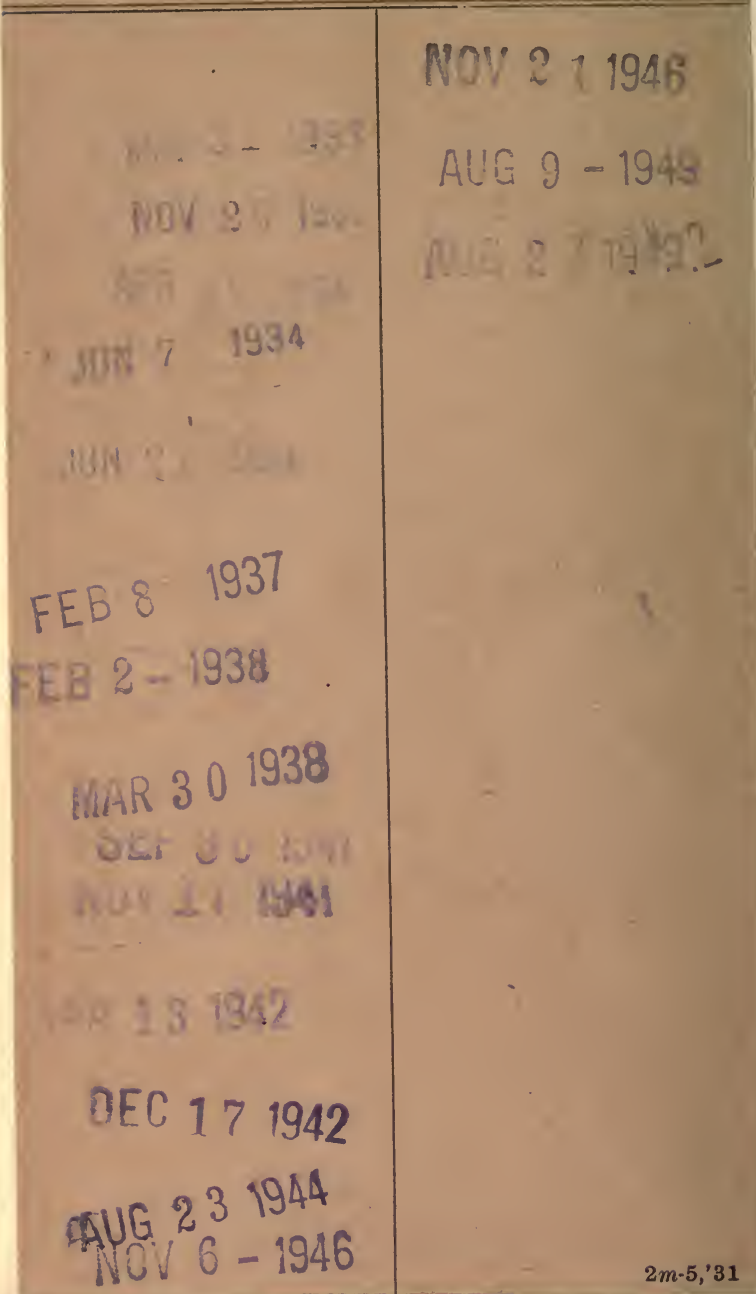


ธ\&، ' $8-2 u$ I
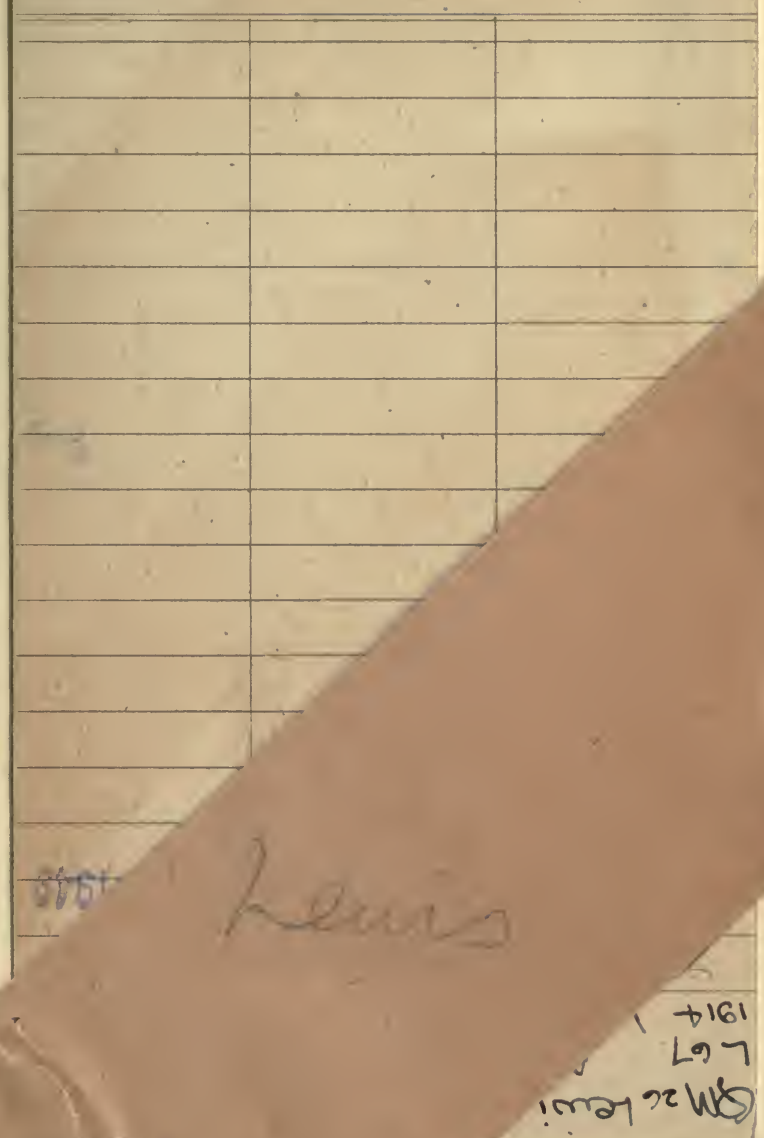

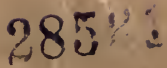


